\title{
The Hoard of Celtic Coins from Deutsch Jahrndorf (Austria, 1855)
}

\author{
Poklad keltských mincí z Deutsch Jahrndorfu (Rakousko, 1855)
}

\author{
Jiří Militký - Melinda Torbágyi
}

Redakci předloženo v ř́ínu 2020, upravená verze v únoru 2021

\begin{abstract}
The Deutsch Jahrndorf (Burgenland, Austria) hoard was discovered in 1855. It contained Bratislava Celtic coinage - gold denominations and silver tetradrachms of the Biatec group. Altogether, 163 coins have been studied either by autopsy or from their earlier publications; originally however, they were surely more numerous. Although the treasure was discovered south of the Danube, $15 \mathrm{~km}$ away from the Bratislava oppidum acropolis, there is no doubt about its direct association with this site. Its contents provide a unique insight into the production of gold denominations, both anepigraphic and with the legends BIATEC or BIAT. Silver tetradrachms of the Biatec group include the majority of known die combinations. The Deutsch Jahrndorf hoard represents a unique source for better understanding the Bratislava coin production. Based on our present state of knowledge of the late La Tène chronology, the hoard was probably concealed in the third quarter of the $1^{\text {st }}$ century BC; a more precise date cannot be established. New discoveries of Roman style constructions on the Bratislava oppidum acropolis help us better understand the phenomenon of relations between the Roman Republic and local Celtic elites; the detailed study of the hoard in question contributes to this topic from the numismatic point of view.
\end{abstract}

Celtic coins, Bratislava, oppidum, hoard, Biatec, stater, tetradrachm

Poklad Deutsch Jahrndorf (Burgenland, Rakousko) byl odkryt v roce 1855. Obsahoval bratislavské keltské mince - zlaté nominály a tetradrachmy skupiny Biatec. Celkem se z tohoto souboru podařilo nashromáždit či alespoň literárně doložit 163 mincí; pưvodní počet nalezených ražeb byl však určitě vyšší. Ačkoliv byl poklad objeven $15 \mathrm{~km}$ jižně od akropole bratislavského oppida, nepochybně s touto lokalitou př́mo souvisí. Díky tomuto nálezu lze jedinečným způsobem sledovat produkci zlatých nominálủ bez nápisủ i s nápisy BIATEC či BIAT. Stříbrné tetradrachmy skupiny Biatec zahrnují většinu známých kombinací razidel. Poklad představuje zcela jedinečný pramen $k$ poznání bratislavské mincovní produkce. Podle dosavadních znalostí lze ukrytí pokladu z Deutsch Jahrndorfu chronologicky zařadit do 3. čtvrtiny 1. století před Kristem, užší datování zatím není možné. Nové archeologické nálezy řimské architektury na akropoli bratislavského oppida umožňují lépe pochopit tamní vztahy mezi římskou republikou a lokálními keltskými elitami. K tomuto tématu se snaži př̌ispět i prededložené detailní zpracování depotu.

Keltské mince, Bratislava, oppidum, poklad, Biatec, stater, tetradrachma

\section{Introduction}

The detailed analysis of old finds of Celtic coins is one of the important tasks of current numismatic research. It is in particular true as far as coin hoards are concerned. As a matter of fact, new finds often slip out of the control of official numismatics and therefore the possibility of adequate evaluation. It is also the case of hoards containing the coins minted in Celtic Bratislava. Paradoxically, although this topic has recently been the subject of considerable attention as will be shown later, most early finds of Bratislava coin hoards have not been newly published since the times of Paulsen (1933, 103-125). Only a small hoard from Schottwien in Lower Austria has recently been restudied (Ruske 2011b). The old finds have been recently augmented by a few small hoards newly discovered in Slovakia (Čambal - Budaj 2009; Budaj - Čambal 2016) as well as by two assemblages whose provenance was not known (Militký 2013a; $2013 b$ ), although later it became clear that the hoard with a new tetradrachm type inscribed LATTV came from the Salzburg region (Röttger 2015, 195, 205).
Moreover, the recent publication of the hoard from the Bratislava castle acropolis is of extraordinary significance (Musilová - Kolníková - Hložek 2015). The assemblage was unearthed during an excavation in the interior of the 'Roman building I'. The gold component of this assemblage allows for a comparison with the Deutsch Jahrndorf assemblage which was until recently the only hoard of Bratislava coinage containing also gold pieces. This latest Bratislava find as well as the recent knowledge of Bratislava oppidum architecture compellingly invite us to make a thorough revision of the Deutsch Jahrndorf hoard.

\section{The find-spot, find circumstances, and study history of the Deutsch Jahrndorf hoard}

The subject of this paper is a coin hoard discovered in the territory of the municipality of Deutsch Jahrndorf 

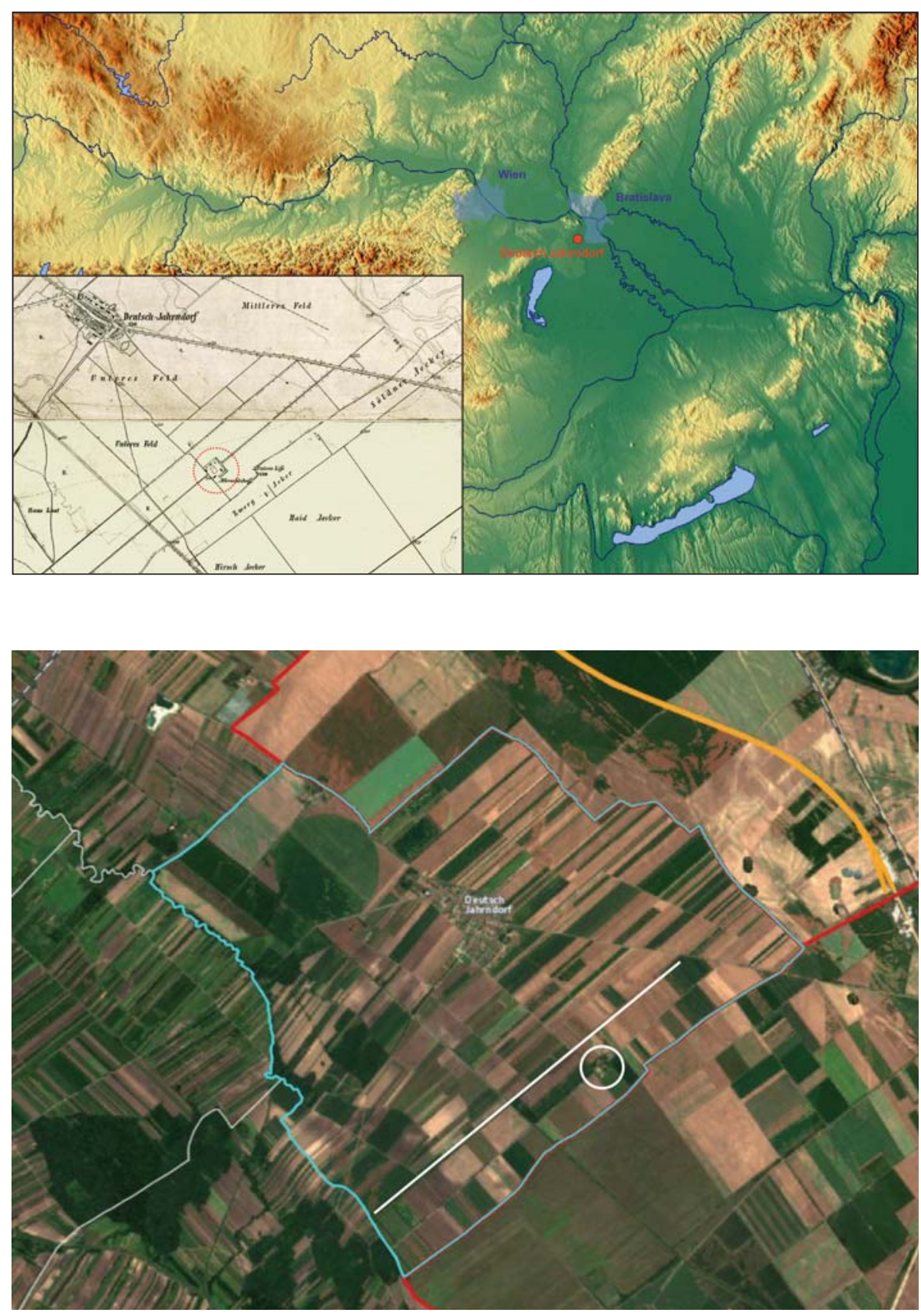

Fig. 1. Map showing the location of Deutsch Jahrndorf (Free Software Foundation). The Karlhof farmstead is indicated with the red dashed circle on the historical map (Archiv des Militärgeographischen Institutes; commons.wikimedia.org). - Obr. 1. Mapa s vyznačením polohy obce Deutsch Jahrndorf (Free Software Foundation). Dvưr Karlhof jen označen červeným přerušovaným kroužkem na historické mapě (Archiv des Militärgeographischen Institutes; commons.wikimedia.org).

Fig. 2. Territory of Deutsch Jahrndorf municipality. The line marks the probable find-spot of the hoard. The Karlhof farmstead is indicated with the white circle (source: Mapy.cz) - Obr. 2. Katastrální území obce Deutsch Jahrndorf s vyznačením linie, ve které byl nález žrejmě odkyt. Dvưr Karlhof jen označen bilým kroužkem (zdroj: Mapy.cz).

(PB Neusiedl am See, Burgenland), ${ }^{1}$ currently adjoining the borders of Hungary and Slovakia. Up until 1921, the village was part of Hungary (named Német-Járfalu or Járfalu). It is located on the flatlands south of the Danube (which meandered considerably in the past) about $15 \mathrm{~km}$ south of the Bratislava castle hill (Fig. 1).

The hoard was discovered in May of 1855 by the farmer Paul Eder digging a boundary ditch between the communal and Archduke's plots of land (Humer 2006, 26). The place of discovery can be located in the surroundings of the Karlhof farmstead (Schmeller 1965, 225) previously called Albrechts Hof, somewhere within the broad strip of the Archduke's fields along the south-

\footnotetext{
${ }^{1}$ In earlier studies, the village's earlier German name Deutsch-Jahrendorf is used.
}

-eastern edge of the municipality territory. The precise find-spot cannot be determined, ${ }^{2}$ though it was probably somewhere along the line of the north-eastern boundary of the Archduke's domain (Fig. 2). It is very clearly visible in the 1856 cadastral plan ('Franziszeischer Kataster') including the information concerning the property. ${ }^{3}$ It seems clear, therefore, that the findspot was located in an entirely flat landscape without

\footnotetext{
${ }^{2}$ The archaeologist Ágost Sötér (1837-1905) allegedly undertook three excavations at the site of discovery in 1885 but failed to find any more coins. I am grateful for the information to Tamas Czuppon (Hansagi Museum, Mosonmagyaróvár).

${ }^{3}$ https://mapire.eu/de/synchron/cadastral/?bbox $=1901057$. $1620685416 \% 2 \mathrm{C} 6102143.041941289 \% 2 \mathrm{C} 1913554.616193167$ $8 \% 2 \mathrm{C} 6109786.744769806 \&$ map-list=1 \&layers=3\%2C4\&rightlayers $=\mathrm{osm}$
} 
prominent landscape features; originally it could have been marked e.g. by vegetation.

The coins were kept in a ceramic vessel which is not preserved and apparently no attention was paid to it at the moment of discovery. It is not clear what happened to the coins immediately after the discovery though there was obviously an effort to sell them. A substantial part of the hoard ended up in the hands of the local teacher who offered them for sale to a jeweller in Bratislava (Seidl 1855, 302). ${ }^{4}$ The coins were saved from being melted down by a literary historian of Bratislava origin, prof. Karl Julius Schröer (1825-1900), ${ }^{5}$ who can therefore be thanked for the preservation of the core of the treasure. In this way, 26 gold and 101 silver coins have been preserved and gone through an administrative procedure (Kupido 1866, 98). ${ }^{6}$ A part of this lot ended up in the numismatic collections in Vienna (41 pcs, Kunsthistorisches Museum Wien - henceforth abbreviated KHM Wien) and in Budapest (86 pcs, Magyar Nemzeti Múzeum - henceforth MNM Budapest) though not even all of these have come down to us. It is sure that the 127 documented pieces do not correspond to the entire hoard; this is clear from the fact that as many as 163 coins are currently associated with the find. It cannot be estimated how many coins were originally present in the hoard. It is even possible, that some of the pieces were melted down, as was still relatively common in the $19^{\text {th }}$ century.

The fact that an unknown part of the hoard became dispersed is well illustrated by pieces from collections other than KHM Wien and the principal lot acquired in MNM Budapest in 1856. These include the collections of Ernst Prinz zu Windisch-Grätz ${ }^{7}$ and K. Niklovits ${ }^{8}$ (cat. nos. 22, 26, 28, 32, 35, 43, 59, 65, 72, 75, 78, 90, 97, $119,129,132,135-136$, and 141), as well as from other private collections. 37 such coins have been documented and it is probable, that some other unprovenanced pieces from various collections may come from this hoard as well.

The collections of the KHM Wien host 42 pieces (Tab. 1), originating almost exclusively from the lot salvaged by J. K. Schröer (41 pcs). ${ }^{9}$ It is not by accident that these include the majority of gold issues (18 pcs) and the best minted and preserved specimens from the silver tetradrachms. The Vienna coin cabinet had the right of pre-emptive selection and only in the second step were the remaining coins from the hoard sent to the MNM Budapest. In Vienna, there is moreover, one coin from the H. F. von Koblitz collection (cat. no. 21) whose provenance from the hoard is nevertheless uncertain.

\footnotetext{
${ }^{4}$ Both the finder and the local teacher obviously kept the discovery secret.

${ }^{5}$ Karl Julius Schröer was a teacher in Bratislava during the years 1851-1860.

6 The same number of coins is mentioned.

7 Two coins from the Windisch-Grätz collection are lost today (cat. nos. 22 and 86).

8 Acquired by the MNM Budapest in 1965.

${ }^{9}$ We are grateful to Dr. K. Vondrovec (KHM Wien) for granting us access to the Viennese part of the treasure and for providing us with its photographs.
}

As has already been stated, the majority of the coins from the hoard are currently kept in the MNM Budapest (Tab. 1). The original lot salvaged by K. J. Schröer was catalogued there in 1856 (inv. nos. 14/1856.A-H and 14/1856.1-77) and is preserved there with the exception of seven tetradrachms. ${ }^{10}$ However, the collections of the MNM Budapest also include other coins from the hoard which arrived there from various private collections over the course of the $20^{\text {th }}$ century. It was in particular the K. Niklovits collection (cat. nos. 32, 35, 43, $65,72,75,78,132$, and 135; for the collection cf. Militký 2015a, 27), including also pieces from the famous Ernst Prinz zu Windisch-Grätz collection (cat. nos. 26, $28,59,90,97,119,129,136$, and 141; for the collection cf. Militký 2015a, 27). Provenance from the said treasure is furthermore recorded for three coins from the M. Dessewffy ${ }^{11}$ collection (cat. nos. 34, 37, and 84), two from the Âldor collection (cat. nos. 96 and 145), and one from the collections of V. v. Renner (cat. no. 117; for the collection cf. Militký 2015a, 27) and Trau (cat. no. 36), all of them currently kept at the MNM Budapest. Altogether, there have been 103 preserved and another 7 unpreserved coins of the Deutsch Jahrndorf hoard in the MNM Budapest collection (Tab. 1).

Individual sparse pieces arrived by various means to the Berlin Münzkabinet (cat. no. 92) and to BNF Paris (cat. no. 139). An origin from the hoard is also claimed in the case of a tetradrachm from the Ch. Röttger collection (cat. no. 110). Another seven coins were present in various private collections but their current whereabouts are unknown. Two of them were documented by means of a photograph (cat. nos. 47 and 69), one by a drawing (cat. no. 104), and four have been described without a depiction (cat. nos. 76, 82, 94, and 154).

It needs to be said that in some cases the provenance of some of the coins from the Deutsch Jahrndorf hoard is not certain (cat. nos. 21, 82, 84, 94, 96-97, and 104). These pieces further illustrate the extent to which the reconstruction of the preserved part of the hoard is complex.

Numerous authors dealt with the Deutsch Jahrndorf hoard in the past. The first short report on the discovery was published by J. Schröer (1855) including also a brief description of the coins. A lengthy description of the hoard was provided by J. V. Seidl (1955, 302-305) in his account of new archaeological finds in the territory of the Austrian Empire. The silver coins from the hoard were analysed by F. S. Kupido who - based on the collection of the Vienna coin cabinet - described 23 pieces and depicted five (Kupido 1866, Taf. 4: 1-5). The find was again dealt with (though not catalogued) by F. Kenner (1895). Its detailed description was provided only by R. Paulsen (1933, 113-115) who published all the gold pieces and a part of the silver coins from various collec-

\footnotetext{
10 These issues probably also included one tetradrachm with the legends AINORIX and one with the legend BVSSVMARVS (Paulsen 1933, 113; Röttger 2019, no. 1-21 / 1259 and no. 18-9 / 1258). These coins are no longer present in the collection and were most probably lost at the end of WW2.

11 The large collection of Celtic coins was published by the collector himself (Dessewffy 1910) and is presently kept at the MNM Budapest.
} 


\begin{tabular}{|l|l|l|c|}
\hline & \multicolumn{1}{|c|}{ AV (cat. nos.) } & \multicolumn{1}{|c|}{ AR (cat. nos.) } & Pcs \\
\hline KHM Wien & $\begin{array}{l}1-3,5-10,15-17,21, \\
23-25,27,29\end{array}$ & $\begin{array}{l}30-31,49,56-57,68,73,80-81,83,87-89,91,93,95, \\
98,101,103,117,137-138,140,151\end{array}$ & $\mathbf{4 2}$ \\
\hline MNM Budapest & $4,11-14,18-20,26,28$ & $\begin{array}{l}32-46,48,50-55,58-67,70-72,74-75,77-79, \\
84-85,90,94,96-97,99-100,102,105-109,111-136, \\
141-150,152-153,156\end{array}$ & 103 \\
\hline $\begin{array}{l}\text { MNM Budapest } \\
\text { (unknown present whereabouts) }\end{array}$ & & $157-163$ & $\mathbf{7}$ \\
\hline MK Berlin & & 92 & $\mathbf{1}$ \\
\hline BNF Paris & & 139 & $\mathbf{1}$ \\
\hline Coll. Ch. Röttger & 110 & $\mathbf{1}$ \\
\hline $\begin{array}{l}\text { Coll. Windisch-Grätz } \\
\text { (unknown present whereabouts) }\end{array}$ & 22 & 86 & $\mathbf{2}$ \\
\hline $\begin{array}{l}\text { Private collection } \\
\text { (unknown present whereabouts) }\end{array}$ & & $47,69,76,82,104,154$ & $\mathbf{6}$ \\
\hline & & 5 & $\mathbf{1 6 3}$ \\
\hline
\end{tabular}

Tab. 1. Deutsch Jahrndorf hoard (1855). Distribution of coins in the collections. - Tab. 1. Poklad z Deutsch Jahrndorfu (1855). Rozpis mincí podle sbirek. tions. In his description of the Budapest collection, he mentioned the presence of 77 tetradrachms but published only single pieces, as a rule not from this lot (cat. nos. 36, 37, 47, 96, 117, and 145). Also, the K. Niklovits collection was published only partially by Paulsen (12 pcs). As a result, the core of the tetradrachm component of the hoard remained completely unknown until very recently.

The find was also repeatedly presented in the following years. Paradoxically it was incorrectly included in the inventory of finds of Celtic coins in Slovakia (Ondrouch 1958, 103, no. 22 and 113, no. 1; 1961, 29, no. $84 ; 1964,50$, no. 84 ) as originating from the site of Jarovce. The village of Jarovce (the present-day borough of Bratislava-Jarovce), founded in the $16^{\text {th }}$ century by Croatian colonists, was called Kroatisch Jahrendorf in the $19^{\text {th }}$ century and so it probably got confused with Deutsch Jahrndorf. There are, moreover, errors in the description of the find circumstances ('while digging graves in the territory of Jarovce') and the brief description mentions the overall number of coins to be ('estimated at') ca. 124 pieces (Ondrouch 1964, 50, no. 84).

Austrian numismatics revisited the hoard in 1984 when its content was briefly listed in one of the volumes of the Die Fundmünzen der römischen Zeit in Österreich project (FRMÖI/2, 327-329, nos. 5/4/1-77, Taf. 1-2). ${ }^{12}$ It is however, only an overview of the coins published already by $R$. Paulsen (1933, 113-115) counting altogether 77 pieces while the unpublished Budapest component was not even mentioned.

The Vienna component of the hoard was entirely republished within the catalogue of Celtic coins in the KHM Wien collections (Dembski 1998, nos. 531-535, 539-540, 542-546, 548-551, 561, 567, 594-595, 597598, 601, 605, 609-610, 614-615, 622-623, 625, 627, 630-631, 634-635, 637-638, 640, 643, 647). It was also presented in the exhibition "Legionsadler und Druidenstab" in Carnuntum (Humer 2006, 26-27, no. 87). The content of the find was briefly summarised by A. Ruske $(2011 a, 62$, no. V4) in his overview of coin treasures dis-

\footnotetext{
12 References to the individual items of this list are systematically provided in the Catalogue; in some instances, the identification is not certain and in one case the coin was not identified at all (FRMÖ I/2, 328, no. 5/4/32).
}

covered in the territory of Austria. The last mention of the hoard so-far can be found in the publication of the most recent coin hoard discovered in Bratislava (Musilová - Kolníková - Hložek 2015, 269-270, Tab. 4) and in the monographic publication of the La Tène period in Eastern Austria (Militký 2020, 313-318, Abb. 9.9).

The so far unpublished database of Ch. Röttger $(2019)^{13}$ is of key importance for a detailed reconstruction of the hoard. The aim of the project is the identification of each documented tetradrachm of the Biatec group and of the corresponding gold denominations. The coins are also classified according to their find-spots. The database precisely tracks the auction history of many coins from the hoard. It also includes unpublished coins from the part of the hoard kept at the MNM Budapest. This documentation, currently kept in the card index of the Institut für Numismatik in Vienna, was created during works on the R. Göbl (1994) monograph. For each of the listed coins, its origin and the entire publication history is tracked. It helped us understand that two coins from the Viennese coin cabinet (Fig. 3), traditionally believed to have come from the Deutsch Jahrndorf hoard, in reality do not.

The first coin which does not come from the Deutsch Jahrndorf treasure is a $1 / 3$-stater of the shell series with the legend BIAT (Militký 2015a, var. 1/3-F/VII:02; inv. no. GR 27109; Fig. 3: 1). The coin has been repeatedly published as a part of the hoard (Kenner 1895, Taf. 1: 10; Paulsen 1933, 76, 114, 155, no. 705, Taf. 29: 705; Ondrouch 1958, Tab. 4: 4; Ondrouch 1961, Tab. XII: 24; FRMÖ I/2, 327, no. 5/4/25, Taf. I: 25; Dembski 1998, 78, no. 538, Taf. 27: 538; Militký 2020, 314-315, Abb. 9.9: 7). The coin was nevertheless first published in 1779 and therefore cannot come from the hoard (Eckhel 1779, Taf. VI: 16).

The other coin not originating from the hoard is a tetradrachm with the legend BIATEC, var. Göbl (1994) II/4-7 (inv. no. GE 27088; Fig. 3: 2). This coin was also repeatedly published as a piece from the hoard (Paulsen 1933, 155, no. 715, Taf. 30: 715; FRMÖ I/2, 328, no.

\footnotetext{
${ }^{13}$ We are deeply indebted to Ch. Röttger for his help with the identification of individual pieces from the hoard.
} 

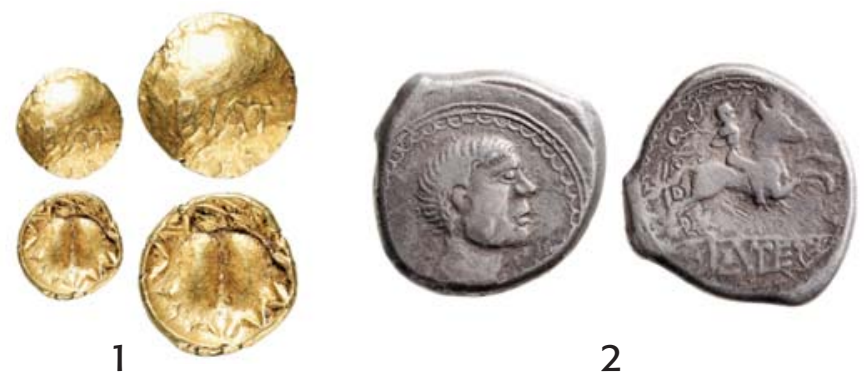

2

Fig. 3. Coins incorrectly associated with the Deutsch Jahrndorf hoard originating from an unknown find-spot: 1 - a 1/3-stater of the shell series with the legend BIAT (Militký 2015a, Var. 1/3-F/VII:02, KHM Wien, inv. no. GR 27109; Paulsen 1933, Taf. 29: 705; Militký 2020, Abb. 9.9: 7); 2 - a tetradrachm of the Biatec group - BIATEC (Göbl 1994, Var. II/4-7, KHM Wien, inv. no. GE 27088; Dembski 1998, no. 607). - Obr. 3. Mince omylem řazené k depotu Deutsch Jahrndorf, pocházejíci z neznámé lokality: 1 - 1/3 statéru mušlové řady s nápisem BIAT (Militký 2015a, var. 1/3-F/VII:02, KHM Wien, inv. Č. GR 27109; Paulsen 1933, Taf. 29: 705); 2 - tetradrachma skupiny Biatec - BIATEC (Göbl 1994, var. II/4-7, KHM Wien, inv. Č. GE 27088; Dembski 1998, č. 607).

5/4/30, Taf. 1: 30; Dembski 1998, 81, no. 607, Taf. 31: 607). The incorrectness of this assumption is demonstrated by its first publication and depiction already in 1840 (Boczek 1840, Taf. III: II).

\section{Catalogue of coins from the hoard}

\section{Southwestern Slovakia, Boii}

\section{AV stater, shell type, var. I.1}

Obv.: irregularly circular smooth clearly delimited bulge with a circular dimple in the centre, at the edge a short line (stylised bird's head?), uneven field around the bulge

Rev.: a marked crescent at the lower edge, the adjoining field around the central dimple covered with irregular rays converging towards the dimple

Ref.: Paulsen 1933, Taf. 28: 682; Dembski 1998, Taf. 28: 548

This coin: Paulsen 1933, 75, 155, no. 682, Taf. 28: 682; Ondrouch 1961, Tab. XII: 5; FRMÖ I/2, 327, no. 5/4/6, Taf. I: 6; Dembski 1998, 78, no. 548, Taf. 28: 548; Röttger 2019, Database no. 70-5 / 1667

1. ${ }^{14} 6.43 \mathrm{~g} ; 16.2 \mathrm{~mm}$ (KHM Wien, inv. no. GR 27119)

AV stater, shell type, var. I.2

Obv.: irregularly circular smooth clearly delimited bulge with a circular central dimple crossed with an arched line (stylised bird's head?); uneven field around the bulge with several parallel rays in one point

Rev.: a marked crescent at the lower edge, the adjoining field around the central dimple covered with regular rays converging towards the central dimple; an oval 'grain' in the rayed field on the right

Ref.: Paulsen 1933, Taf. 28: 683; Dembski 1998, Taf. 28: 545

\footnotetext{
${ }^{14}$ The coins whose catalogue number is printed in bold are represented in figures $4 a-4 i$.
}

This coin: Paulsen 1933, 75, 155, no. 683, Taf. 28: 683; Ondrouch 1961, Tab. XII: 6; FRMÖ I/2, 327, no. 5/4/7, Taf. I: 7; Dembski 1998, 78, no. 545, Taf. 28: 545; Röttger 2019, Database no. 78-1 / 1668

2. $6.45 \mathrm{~g} ; 14.4 \mathrm{~mm}$ (KHM Wien, inv. no. GR 27120)

AV stater, shell type, var. I.3

Obv.: irregularly circular smooth, clearly delimited bulge with an oval central dimple and a stylised bird's head (?) inside it; uneven field around the bulge

Rev.: a marked crescent at the lower edge, smooth adjoining field around the central dimple

Ref.: Paulsen 1933, Taf. 28: 681; Dembski 1998, Taf. 28: 551

This coin: Paulsen 1933, 75, 155, no. 681, Taf. 28: 681; Ondrouch 1961, Tab. XII: 4; FRMÖ I/2, 327, No. 5/4/5, Taf. I: 5; Dembski 1998, 79, no. 551, Taf. 28: 551; Röttger 2019, Database No. 70-7 / 1666

3. $6.18 \mathrm{~g} ; 14.5 \mathrm{~mm}$ (KHM Wien, inv. no. GR 27122)

AV stater, shell type, var. II.1

Obv.: irregular oval smooth, clearly delimited bulge with an oval central dimple, a fine wavy line around the bulge, even field around the bulge

Rev.: a marked crescent at the lower edge, the adjoining field around the central dimple is covered with regular rays converging towards the dimple, a 'wedge shaped' arrow in the middle of the rays pointing towards the dimple

Ref.: Paulsen 1933, Taf. 28: 686

This coin: Paulsen 1933, 114, 155, Taf. 28: 686; Ondrouch 1961, Tab. XII: 9; FRMÖ I/2, 327, no. 5/4/10; Röttger 2019, Database no. 70-1 / 1671

4. $6.74 \mathrm{~g} ; 15.4 / 15.1 \mathrm{~mm}$ (MNM Budapest, inv. no. 14/ 1856.H)

AV stater, shell type, var. II.2

Obv.: irregular oval smooth clearly delimited bulge with a circular central dimple; uneven field around the bulge Rev.: a marked crescent at the lower edge, the adjoining field is covered with rays symmetrically converging towards the 'wedge shaped' arrow in the middle, small irregular pellets on the rays

Ref.: Paulsen 1933, Taf. 28: 684-685; Castelin 1965, Taf. 3: 29; Dembski 1998, Taf. 27: 543-544.

This coin: Paulsen 1933, 75, 114, 155, no. 685, Taf. 28: 685; Ondrouch 1961, Tab. XII: 8; FRMÖ I/2, 327, no. 5/4/9, Taf. I: 9; Dembski 1998, 78, no. 543, Taf. 27: 543; Fröhlich 2016, 6-7, no. 5, Fig. 3: 5; Röttger 2019, Database no. 70-4 / 1670; Militký 2020, 314-315, Abb. 9.9: 1

5. 6.48 g; 15.7 mm (KHM Wien, inv. no. GR 27115)

-, This coin: Paulsen 1933, 75, 114, 155, no. 684, Taf. 28: 684; Ondrouch 1961, Tab. XII: 7; Castelin 1965, Taf. 3: 29; FRMÖ I/2, 327, no. 5/4/8, Taf. I: 8; Dembski 1998, 78, no. 544, Taf. 27: 544; Röttger 2019, Database no. $70-3$ / 1669

6. 6.47 g; 16.4 mm (KHM Wien, inv. no. GR 27114)

AV stater, shell type, var. II.3

Obv.: irregular oval delimited bulge with a stylised bird's head (?); uneven field around the bulge with hints of rays 
Rev.: marked crescent at the lower edge, the adjoining field is symmetrically covered with rays converging towards a shallow central dimple and a 'wedge shaped' arrow in the middle of the rays; small irregular pellets on the rays, in the field on the left and on the right two oval 'grains'

Ref.: Paulsen 1933, Taf. 28: 678-679; Castelin 1965, Taf. 3: 32; Dembski 1998, Taf. 28: 549-550; Rudnicki 2011, 106, Fig. 5-6; Musilová - Kolníková - Hložek 2015, Fig. 13: 15

This coin: Paulsen 1933, 75, 114, no. 1431/1911; FRMÖ I/2, 327, no. 5/4/3; Dembski 1998, 78, no. 542, Taf. 27: 542; Röttger 2019, Database no. 69-3 / 1685 7. $6.49 \mathrm{~g} ; 15.8 \mathrm{~mm}$ (KHM Wien, inv. no. GR 27116)

-, This coin: Paulsen 1933, 75, 154, no. 679, Taf. 28: 679; Ondrouch 1961, Tab. XII: 2; Castelin 1965, Taf. 3: 32; FRMÖ I/2, 327, no. 5/4/2, Taf. I: 2; Dembski 1998, 78, no. 549, Taf. 28: 549; Rudnicki 2011, 106, Fig. 6; Röttger 2019, Database no. 69-6 / 1664

8. $6.43 \mathrm{~g} ; 16.0 \mathrm{~mm}$ (KHM Wien, inv. no. GR 27117)

-, This coin: Paulsen 1933, 75, 114, 154, no. 678, Taf. 28: 678; Ondrouch 1961, Tab. XII: 1; FRMÖ I/2, 327, no. 5/4/1, Taf. I: 1; Dembski 1998, 78, no. 550, Taf. 27: 550; Rudnicki 2011, 106, Fig. 5; Röttger 2019, Database no. 69-5 / 1663; Militkú 2020, 314-315, Abb. 9.9: 2 9. $6.42 \mathrm{~g} ; 16.8 \mathrm{~mm}$ (KHM Wien, inv. no. GR 27111)

\section{AV stater, shell type, var. II.4}

Obv.: eccentrically located irregular oval smooth delimited bulge; uneven field around the bulge with hints of rays

Rev.: a marked crescent at the lower edge, the adjoining field is covered with rays symmetrically converging towards a shallow central dimple and a 'wedge shaped' arrow in the middle of the rays; small irregular pellets on the rays, on the left and on the right in the rayed field two hardly visible 'grains'

Ref.: Paulsen 1933, Taf. 28: 680; Dembski 1998, Taf. 28: 546

This coin: Paulsen 1933, 75, 155, no. 680, Taf. 28: 680; Ondrouch 1961, Tab. XII: 3; FRMÖ I/2, 327, no. 5/4/4, Taf. I: 4; Dembski 1998, 78, no. 546, Taf. 28: 546; Röttger 2019, Database no. 69-7 / 1665

10. $6.44 \mathrm{~g} ; 16.9 \mathrm{~mm}$ (KHM Wien, inv. no. GR 27121)

\section{AV stater, shell type, var. III.1}

Obv.: irregular oval clearly delimited bulge, a stylised hand at its upper edge, uneven field around the bulge Rev.: a marked crescent at the lower edge, the adjoining field around the central dimple is covered with almost imperceptible rays converging towards the central dimple Ref.: Paulsen 1933, Taf. 28: 687

This coin: Paulsen 1933, 114, 155, Taf. 28: 687; Ondrouch 1961, Tab. XII: 10; FRMÖ I/2, 327, no. 5/4/11; Röttger 2019, Database no. 71-1 / 1672

11. $6.63 \mathrm{~g} ; 15.7 / 14.9 \mathrm{~mm}$; $9^{\mathrm{h}}$ (MNM Budapest, inv. no. $14 / 1856 . \mathrm{F})$

\section{AV stater, shell type, var. IV.1}

Obv.: irregular oval smooth indistinctly delimited bulge, a stylised hand at its upper edge and a large pellet beneath it; uneven field around the bulge
Rev.: a prominent crescent at the lower edge, the adjoining field is covered with marked organised rays converging towards the central dimple

Ref.: Paulsen 1933, Taf. 29: 692

This coin: Paulsen 1933, 114, 155, Taf. 29: 692; Ondrouch 1961, Tab. XII: 15; FRMÖ I/2, 327, no. 5/4/16; Röttger 2019, Database no. 71-5 / 1677

12. $6.51 \mathrm{~g} ; 15.6 / 14.7 \mathrm{~mm} ; 6^{\mathrm{h}}$ (MNM Budapest, inv. no. 14/1856.B)

\section{AV stater, shell type, var. IV.2}

Obv.: irregular oval smooth indistinctly delimited bulge, a stylised hand at its upper edge and a large pellet beneath it, uneven field around the bulge

Rev.: a marked crescent at the lower edge, the adjoining field is covered with marked organised rays converging towards the central dimple

Ref.: Paulsen 1933, Taf. 28: 688 and 690

This coin: Paulsen 1933, 114, 155, Taf. 28: 690; Ondrouch 1961, Tab. XII: 13; FRMÖ I/2, 327, no. 5/4/14; Röttger 2019, Database no. 71-6 / 1675

13. $6.49 \mathrm{~g} ; 15.0 / 14.5 \mathrm{~mm} ; 1^{\mathrm{h}}$ (MNM Budapest, inv. no. $14 / 1856 . A)$

-, This coin: Paulsen 1933, 114, 155, Taf. 28: 688; Ondrouch 1961, Tab. XII: 11; FRMÖ I/2, 327, no. 5/4/12; Röttger 2019, Database no. 71-7 / 1673.

14. $6.49 \mathrm{~g} ; 14.6 / 14.3 \mathrm{~mm} ; 11^{\mathrm{h}}$ (MNM Budapest, Inv. no. $14 / 1856 . C)$

\section{AV stater, shell type, var. IV.3}

Obv.: irregular oval smooth indistinctly delimited bulge, a stylised hand at its upper edge and a large pellet beneath it, uneven field around the bulge

Rev.: a marked crescent at the lower edge, the adjoining field is covered with marked organised rays converging towards the central dimple, an oval 'grain' in the field on the left

Ref.: Paulsen 1933, Taf. 28: 689; Dembski 1998, Taf. 27: 533

This coin: Paulsen 1933, 76, 155, no. 689, Taf. 28: 689; Ondrouch 1961, Tab. XII: 12; FRMÖ I/2, 327, no. 5/4/13, Taf. I: 13; Dembski 1998, 78, no. 533, Taf. 27: 533; Röttger 2019, Database no. 71-10 / 1674; Militký 2020, 314-315, Abb. 9.9: 4

15. 6.46 g; $16.1 \mathrm{~mm}$ (KHM Wien, inv. no. GR 27118)

AV stater, shell type, var. V.1

Obv.: irregular oval smooth indistinctly delimited bulge, a stylised hand at its upper edge and three large pellets beneath, uneven field around the bulge

Rev.: a marked crescent at the lower edge, the adjoining field is covered with bunches of rays in various directions, converging towards the central dimple, an oval 'grain' in the field on the right

Ref.: Paulsen 1933, Taf. 18: 387, 28: 693 (identical dies) This coin: Paulsen 1933, 76, 114, 155, no. 693, Taf. 28: 693; Ondrouch 1961, Tab. XII: 16; FRMÖ I/2, 327, no. 5/4/17, Taf. I: 17; Dembski 1998, 78, no. 531, Taf. 27: 531; Röttger 2019, Database no. 72-1 / 1678; Militký 2020, 314-315, Abb. 9.9: 3.

16. $6.51 \mathrm{~g} ; 15.8 \mathrm{~mm}$ (KHM Wien, inv. no. GR 27112) 


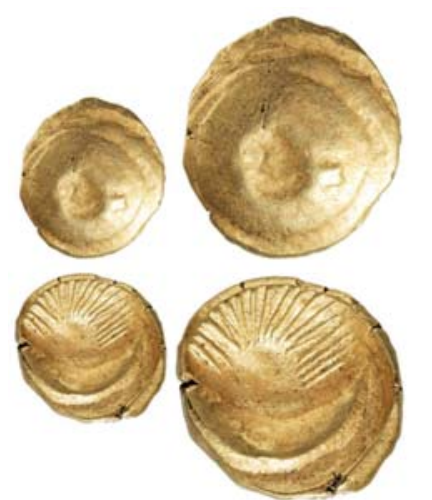

1

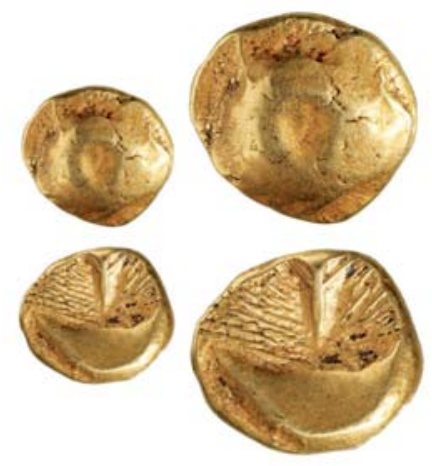

5

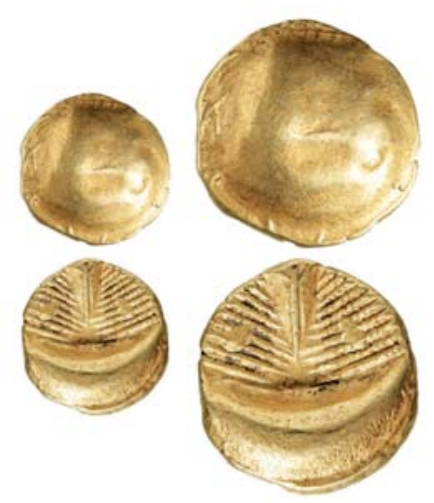

8

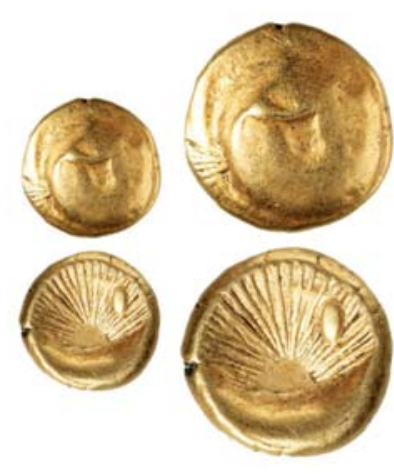

2

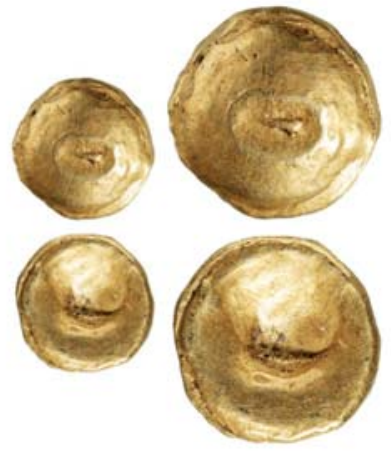

3

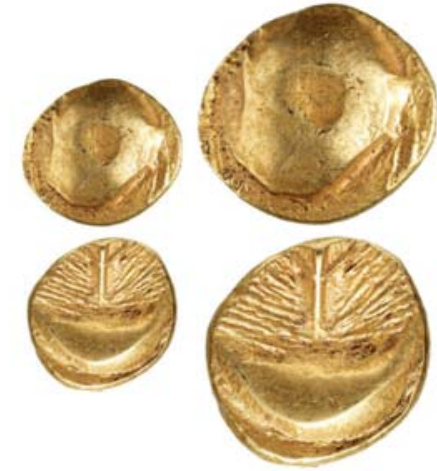

6
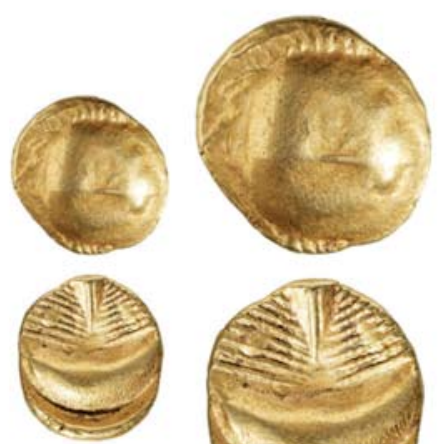

9

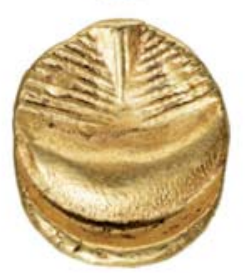

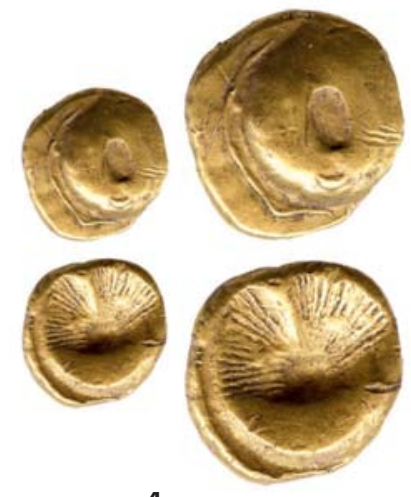

4
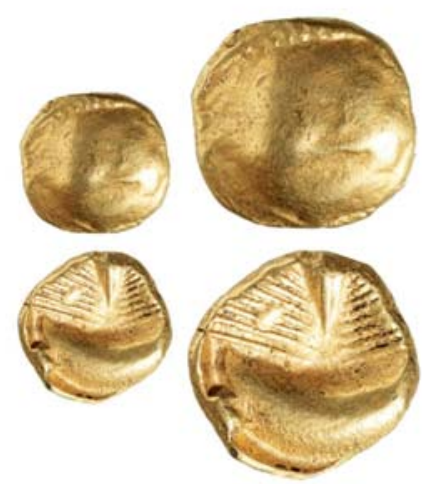

7
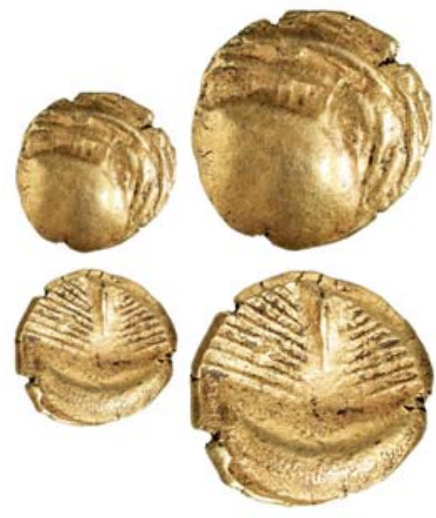

10

Fig. 4a. Deutsch Jahrndorf. Coins from the hoard (the numbers correspond to catalogue; 1:1.5; photos by J. Militký and KHM Wien). - Obr. 4a. Deutsch Jahrndorf. Mince z pokladu (Č́sla odpovidaji katalogu; 1: 1,5; foto J. Militký a KHM Wien).

\section{AV stater, shell type, var. VI.1}

Obv.: irregular oval smooth indistinctly delimited bulge, a stylised hand at its upper edge and beneath it a large pellet and two crescents at the sides, uneven field around the bulge

Rev.: a marked crescent at the lower edge, the adjoining field is covered with marked organised rays converging towards the central dimple; an oval 'grain' in the rayed field on the right

Ref.: Paulsen 1933, Taf. 28: 694; Castelin 1965, Taf. 3: 30; Musilová - Kolníková - Hložek 2015, Fig. 13: 14; Röttger 2020, 24, as Abb. 4 (right)
This coin: Paulsen 1933, 76, 114, 155, no. 694, Taf. 28: 694; Ondrouch 1961, Tab. XII: 17; Castelin 1965, Taf. 3: 30; FRMÖ I/2, 327, no. 5/4/18, Taf. I: 18; Dembski 1998, 78, no. 532, Taf. 27: 532; Röttger 2019, Database no. $74-3$ / 1679

17. $6.47 \mathrm{~g} ; 14.7 \mathrm{~mm}$ (KHM Wien, inv. no. GR 27113)

AV stater, shell type, var. VII.1

Obv.: irregular oval smooth indistinctly delimited bulge with a pellet in the centre, uneven field around the bulge Rev.: a marked crescent at the lower edge, the adjoining field is covered with marked organised rays converging 
towards the central dimple, oval 'grain' in the field on the right

Ref.: Paulsen 1933, Taf. 28: 691

This coin: Paulsen 1933, 114, 155, no. 691, Taf. 28: 691; Ondrouch 1961, Tab. XII: 14; FRMÖ I/2, 327, no. 5/4/15; Röttger 2019, Database no. 71-9 / 1676

18. $6.46 \mathrm{~g} ; 14.5 / 14.2 \mathrm{~mm}$; $6^{\mathrm{h}}$ (MNM Budapest, inv. no. $14 / 1856 . G)$

\section{AV 1/3-stater, shell series, var. Militký (2015a)}

\section{1/3-E/VI:02}

Obv.: smooth oval markedly delimited bulge, the field around the bulge is covered with disorganised lines Rev.: two facing bulges with a straight base, two horizontal 'grains' below them, diagonal lines at the bottom, open zig-zag line around the circumference

Ref.: Paulsen 1933, Taf. 29: 702; Militký 2015a, TAB. 4: 1/3-E/VI:02

This coin: Paulsen 1933, 76, 155, no. 702, Taf. 29: 702; Ondrouch 1961, Tab. XII: 21; FRMÖ I/2, 327, no. 5/4/22; Röttger 2019, Database no. 64-1 / 1680

19. $2.25 \mathrm{~g} ; 12.1 / 11.9 \mathrm{~mm}$ (MNM Budapest, inv. no. $14 / 1856 . \mathrm{E})$

\section{AV 1/3-stater, shell series, var. Militký (2015a) \\ 1/3-F/VII:01}

Obv.: smooth oval indistinctly delimited bulge, uneven field around the bulge

Rev.: two facing oval bulges, a 'grain' beneath each bulge, slanting lines below, enclosed zig-zag line around the circumference

Ref: Paulsen 1933, Taf. 29: 703; Militký 2015a, TAB. 4: 1/3-F/VII:01.

This coin: Paulsen 1933, 76, 155, no. 703, Taf. 29: 703; Ondrouch 1961, Tab. XII: 22; FRMÖ I/2, 327, no. 5/4/ 23; Militký 2015a, 66, TAB. 4: 1/3-F/VII:01 (P 703); Fröhlich 2016, 6-7, no. 6, Fig. 3: 6; Röttger 2019, Database no. 65-1 / 1681

20. $2.10 \mathrm{~g} ; 11.6 / 11.3 \mathrm{~mm}$ (MNM Budapest, inv. no. $14 / 1856 . \mathrm{D})$

-, This coin: Dembski 1998, 79, no. 561, Taf. 28: 561; Rudnicki 2011, 108, Fig. 7; Röttger 2019, Database no. 66-1 / 1687

Comment: The origin of this coin is not certain

21. $2.14 \mathrm{~g}$; 11.0/12.0 mm (KHM Wien - Coll. H. F. von Koblitz /2. 8. 1919 Eger, Wien/, inv. no. GR 38961)

AV 1/3-stater, shell series, var. impossible to define

Ref.: -

This coin: Fiala 1900, 201, no. 2821; Paulsen 1933, 76, 115, no. 2024; FRMÖ I/2, 327, no. 5/4/26; Röttger 2019, Database no. 68-4 / 1686

22. 2.10 g; 12 mm (Coll. Ernst Prinz zu Windisch-Grätz, currently lost)

AV 1/8-stater, shell series, var. Militký (2015a) 1/8-XIII:01

Obv.: smooth irregular oval indistinctly delimited bulge, uneven field around the bulge

Rev.: a triangular motif without a pointed termination with two pairs of rays, below an arched line and indistinct pellets (?)
Ref.: Paulsen 1933, Taf. 29: 706-708; Castelin 1965, Taf. 4: 56; Dembski 1998, Taf. 28: 567; Militký 2015a, TAB. 7: 1/8-XIII:01.

This coin: Paulsen 1933, 77, 114, 155, no. 706, Taf. 29: 706; Ondrouch 1961, Tab. XII: 25; Castelin 1965, Taf. 4: 56; FRMÖ I/2, 328, no. 5/4/27, Taf. I: 27; Dembski 1998, 79, no. 567, Taf. 28: 567; Militký 2015a, 72, TAB. 7: 1/8-XIII:01 (P 706); Röttger 2019, Database no. 63-4 / 1682; Militký 2020, 314-315, Abb. 9.9: 5

23. 0.87 g; 9.7 mm (KHM Wien, inv. no. GR 27110)

AV stater, shell type - BIATEC, var. B-Ia (1-1)

Obv.: irregular smooth indistinctly delimited bulge, a stylised hand at its upper edge and a large pellet on the right; beneath the hand BIATEC

Rev.: at the lower edge a marked crescent, the adjoining field is covered with marked organised rays converging towards the central dimple, on the right in the rayed field a die crack

Ref.: Paulsen 1933, Taf. 29: 696-699; Göbl 1994, Taf. 9: 2-3; Dembski 1998, Taf. 27: 534-535; Musilová-Kolníková - Hložek 2015, Fig. 13: 2-3

This coin: Paulsen 1933, 75, 114, 155, no. 697, Taf. 29: 697; Ondrouch 1961, Tab. XII: 20; FRMÖ I/2, 327, no. 5/4/21, Taf. I: 21; Göbl 1994, Taf. 9: 2; Dembski 1998, 78, no. 534, Taf. 27: 534; Röttger 2019, Database no. 45-6 / 393

24. $6.48 \mathrm{~g} ; 16.0 / 15.4 \mathrm{~mm}$ (KHM Wien, inv. no. GR 27106)

-, This coin: Kenner 1895, Taf. III: 9; Paulsen 1933, 75, 114, 155, no. 696, Taf. 29: 696; Ondrouch 1961, Tab. XII: 19; FRMÖ I/2, 327, no. 5/4/20, Taf. I: 20; Göbl 1994, Taf. 9: 3; Dembski 1998, 78, no. 535, Taf. 27: 535; Göbl 1994, Taf. 9: 3; Röttger 2019, Database no. 45-2 / 392; Militký 2020, 314-315, Abb. 9.9: 6

25. 6.47 g; 15.6 mm (KHM Wien, inv. no. GR 27105)

AV stater, shell type - BIATEC, var. B-Ic (3-2)

Obv.: irregular smooth indistinctly delimited bulge, at its upper edge a stylised hand, on the right a large pellet with rays directed upwards, beneath the hand BIATEC Rev.: at the lower edge a marked crescent, the adjoining field is covered with marked organised rays converging towards the central dimple

Ref.: Paulsen 1933, Taf. 29: 695; Castelin 1965, Taf. 3: 31

This coin: Fiala 1891, 9, no. 56, Taf. I: 11; Fiala 1900, 201, no. 2820, Taf. II: 2820; Paulsen 1933, 75, 114 , 155, no. 695, Taf. 29: 695; Ondrouch 1961, Tab. XII: 18; Castelin 1965, Taf. 3: 31; Pink 1974, Taf. IV: 66; FRMÖ I/2, 327, no. 5/4/19; Röttger 2019, Database no. 45-4 / 391

26. 6.46 g; 16.7 mm (MNM Budapest - Coll. Ernst Prinz zu Windisch-Grätz and K. Niklovits, inv. no. N I 5405)

AV 1/3-stater, shell series - BIAT, var. Militký (2015a) 1/3-F/VII:02

Obv.: smooth oval markedly delimited bulge, on the bulge legend BIAT, uneven field around the bulge

Rev.: two facing oval bulges, a 'grain' beneath either bulge, beneath a slanting line, around the circumference a zig-zag line 


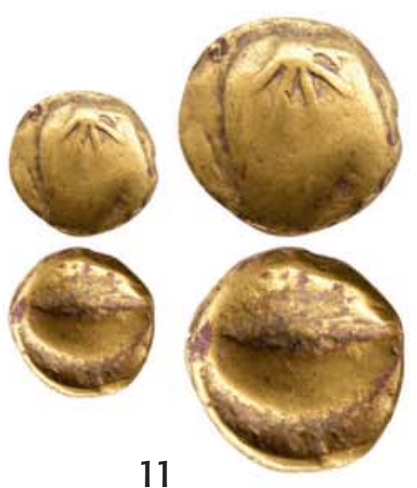

11

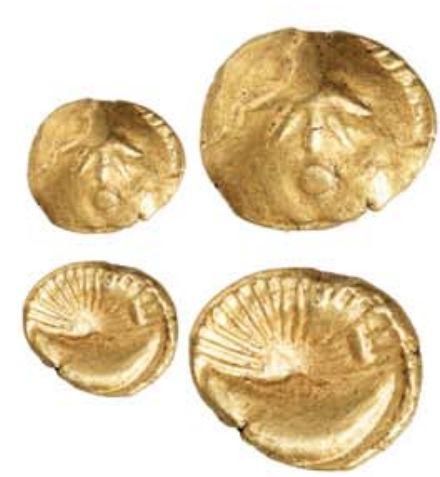

15

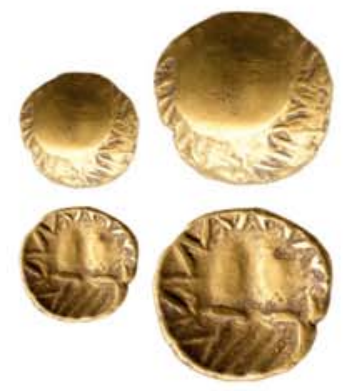

19

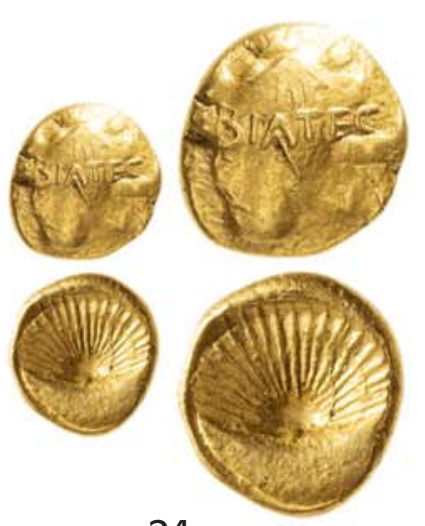

24

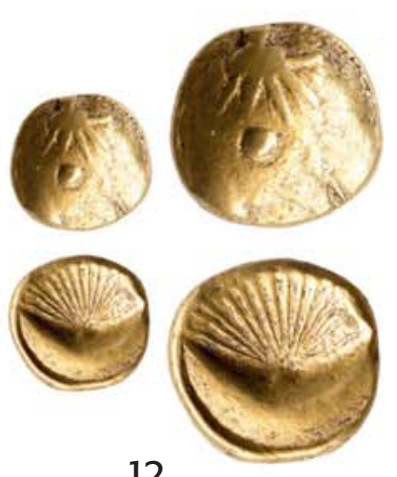

12
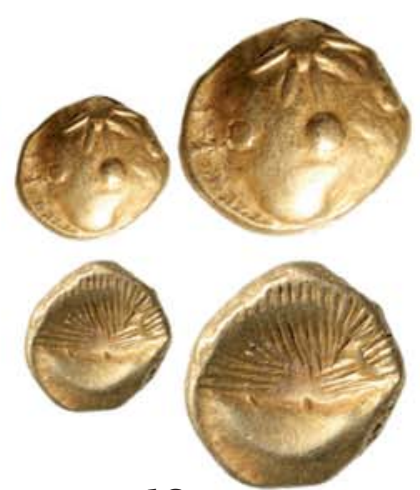

16

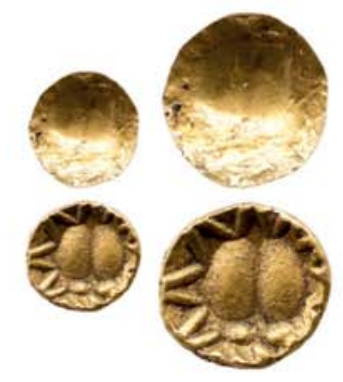

20
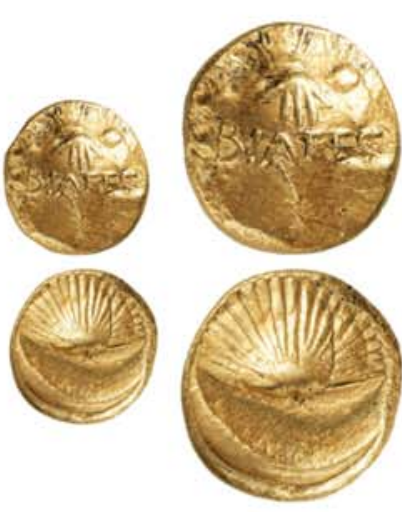

25
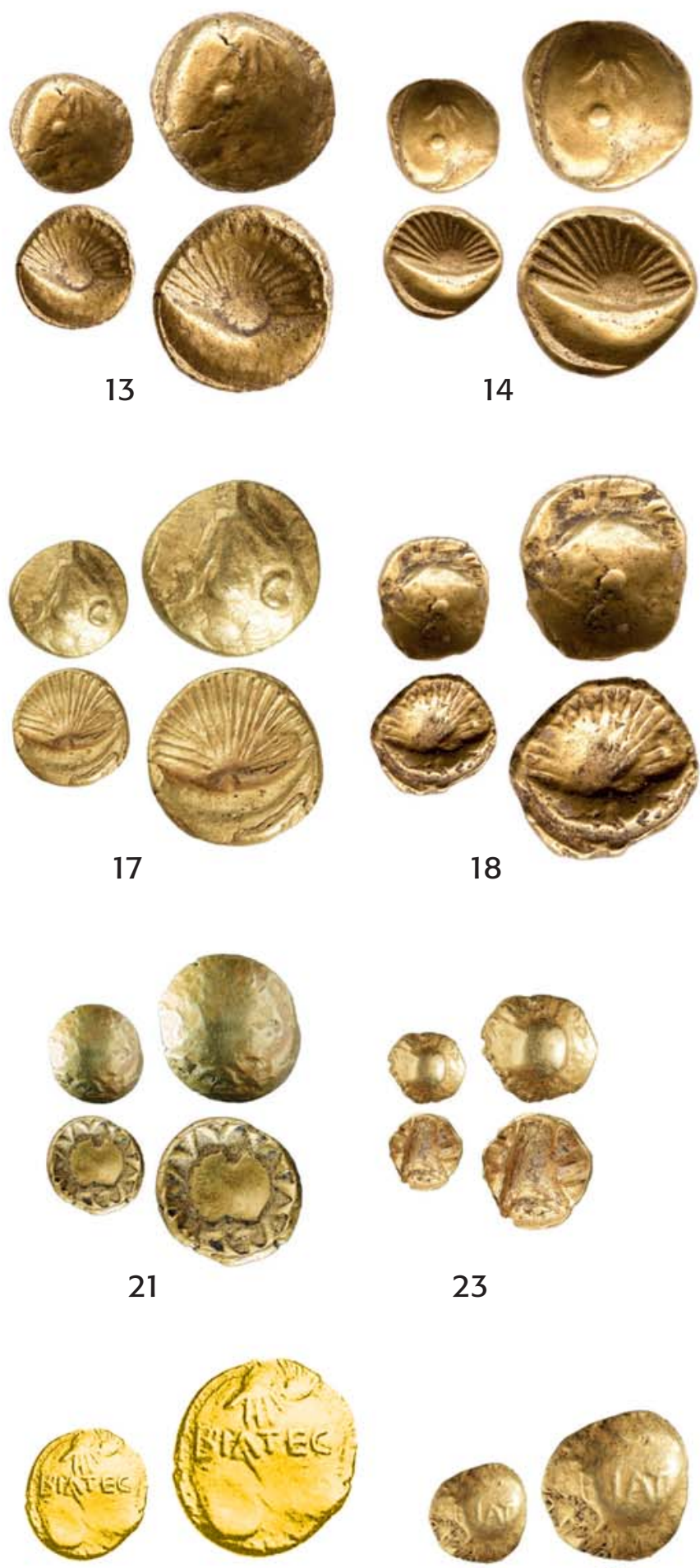

17

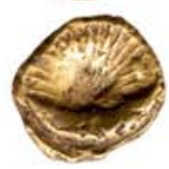

18
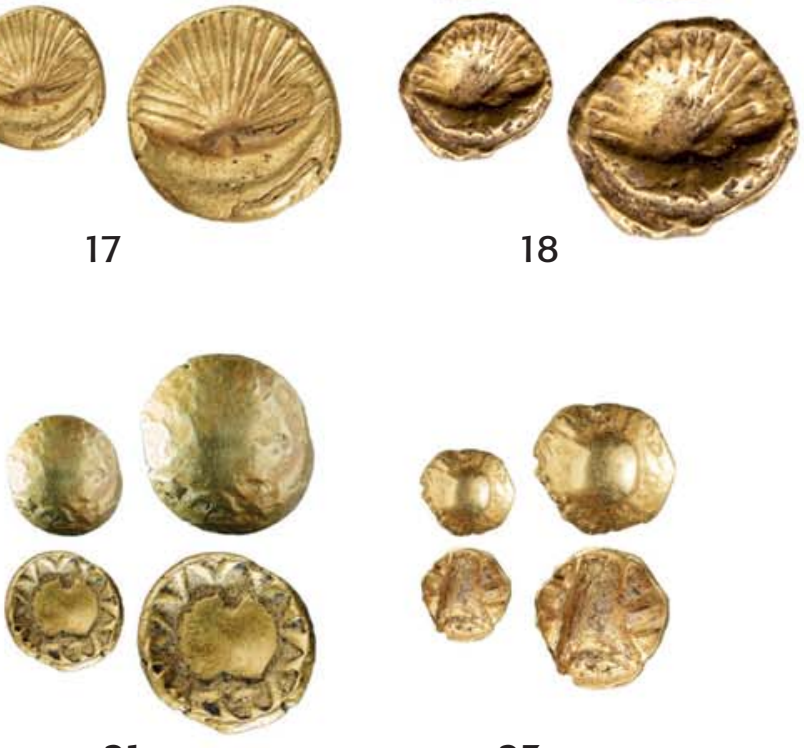

23
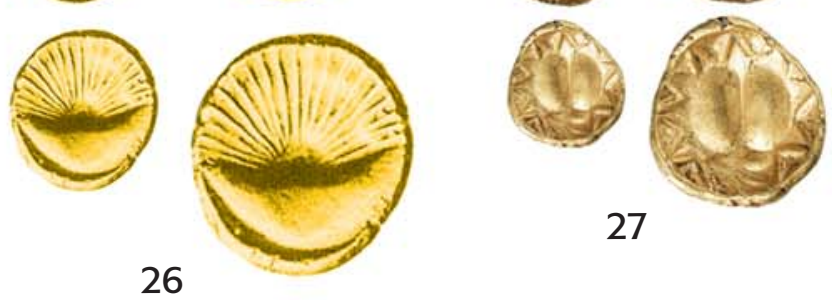

Fig. $\mathbf{4} \boldsymbol{b}$. Deutsch Jahrndorf. Coins from the hoard (the numbers correspond to the catalogue; 1:1.5; photos by J. Militky and KHM Wien). - Obr. $\mathbf{4 b}$. Deutsch Jahrndorf. Mince z pokladu (Č́sla odpovidají katalogu; $1:$ 1,5; foto J. Militký a KHM Wien). 
Ref.: Paulsen 1933, Taf. 29: 704-705; Dembski 1998, Taf. 27: 538; Göbl 1994, Taf. 9: 4; Militký 2015a, TAB. 4: 1/3-F/VII:02

This coin: Paulsen 1933, 76, 114, 155, no. 704, Taf. 29: 704; Ondrouch 1958, Tab. XII:4: 3; Ondrouch 1961, Tab. XII: 23; FRMÖ I/2, 327, no. 5/4/24, Taf. I: 24; Göbl 1994, Taf. 9: 4; Dembski 1998, 78, no. 539, Taf. 27: 539; Röttger 2019, Database no. 44-3 / 398

27. 2.14 g; 13.0 mm (KHM Wien, inv. no. GR 27107)

AV 1/8-stater, shell series - BIAT, var. Militký (2015a)

\section{1/8-XIV:01}

Obv.: smooth undelimited bulge, on the bulge legend BIAT

Rev.: triangular motif without the pointed termination with two pairs of rays, arched line below

Ref.: Paulsen 1933, Taf. 29: 709-710; Castelin 1965 , Taf. 4: 58; Göbl 1994, Taf. 9: 6; Dembski 1998, Taf. 27: 540; Militký 2015a, TAB. 7: 1/8-XIV:01

This coin: Fiala 1891, 10, no. 57, Taf. I: 12; Fiala 1900 , 201, no. 2822; Paulsen 1933, 77, 114, 155, Taf. 29: 710; Ondrouch 1958, Tab. XII:4: 6; Ondrouch 1961, Tab. XII: 27; FRMÖ I/2, 328, no. 5/4/29; Göbl 1994, Taf. 9: 6; Militký 2015a, 72, TAB. 7: 1/8-XIV:01 (P 710); Röttger 2019, Database no. 43-2 / 401

28. 0.83 g; $8.7 / 9.7 \mathrm{~mm} ; 6^{\mathrm{h}}$ (MNM Budapest - Coll. Ernst Prinz zu Windisch-Grätz and K. Niklovits, inv. no. N I 5413)

-, This coin: Kenner 1895, Taf. III: 11; Paulsen 1933, 77, 114, 155, no. 709, Taf. 29: 709; Ondrouch 1958, Tab. XII:4: 5; Ondrouch 1961, Tab. XII: 26; Castelin 1965, Taf. 4: 58; FRMÖ I/2, 328, no. 5/4/28, Taf. I: 28; Göbl 1994, Taf. 9: 6; Dembski 1998, 78, no. 540, Taf. 27: 540; Militký 2015a, 72, TAB. 7: 1/8-XIV:01 (P 709); Röttger 2019, Database no. 43-3 / 400; Militký 2020, 314-315, Abb. 9.9: 8

29. 0.80 g; $9.1 \mathrm{~mm}$ (KHM Wien, inv. no. GR 27108)

AR tetradrachm, Biatec group - AINORIX, var. Göbl (1994) I/1-26

Obv.: male (?) youth bust to left, on the left a branch terminating in pellets (mistletoe?) on the right a wavy twisted line, marked border of dots

Rev.: standing Harpy to the right, looking left, on the right vertically between two lines AINO, below between two lines XIR, marked border of dots

Ref.: Kupido 1866, Taf. 4: 5; Paulsen 1933, Taf. 34: 802805; Ondrouch 1958, no. 246; Göbl 1994, Taf. 2: I/126; Dembski 1998, Taf. 34: 642-643; Kostial 2003, no. 61; Ziegaus 2010, no. 484

This coin: Paulsen 1933, 158, no. 803, Taf. 34: 803; FRMÖ I/2, 329, no. 5/4/75, Taf. 2: 75; Dembski 1998, 82, no. 643, Taf. 34: 643; Röttger 2019, Database no. $1-11 / 493$

30. $16.80 \mathrm{~g} ; 27.0 / 23.9 \mathrm{~mm} ; 2^{\text {h }}$ (KHM Wien, inv. no. GR 27102)

\section{AR tetradrachm, Biatec group - BIATEC,} var. Röttger (2019) II/1 (1)B-1B

Obv.: male and female head to the right (Honos and Virtus), above the heads $\mathrm{V}$-shaped bunches of lines, right below leaf, border of smooth line and arches turned inwards
Rev.: naked rider on horse to the right, rein in the left hand, in the right hand a branch terminating in pellets (mistletoe?), resting on his shoulder, right arm is bent between the horseman's chest and horse's neck, in exergue between two lines BIATEC, leaf before, below, and behind the horse, border of smooth line and arches turned inwards

Ref.: Dessewffy 1910, Tab. V: 142; Paulsen 1933, Taf. 30: 718-721; Ondrouch 1958, nos. 25-28, 30, 40-41; Göbl 1994, Taf. 2: II/1 (1)-1:1; Dembski 1998, Taf. 30: 597; Kostial 2003, no. 62

This coin: Paulsen 1933, 155, no. 719, Taf. 30: 719; FRMÖ I/2, 328, no. 5/4/33, Taf. 1: 33; Dembski 1998, 80, no. 597, Taf. 30: 597; Röttger 2019, Database no. 3-16 / 410; Militký 2020, 314-315, Abb. 9.9: 9

31. $17.15 \mathrm{~g} ; 27.1 \mathrm{~mm}$; $6^{\text {h }}$ (KHM Wien, inv. no. GR 27085)

-, This coin: FRMÖ I/2, 328, no. 5/4/37 (?); Röttger 2019, Database no. 3-19 / 1482

32. $17.15 \mathrm{~g} ; 25.9 / 26.7 \mathrm{~mm} ; 7^{\text {h }}$ (MNM Budapest - Coll. K. Niklovits, inv. no. N I 4532)

-, This coin: Bíró-Sey 1987, 490, Fig. 4A; Göbl 1994, Taf. 2: II/1(1)-1:1, 9: 8; Röttger 2019, Database no. 3-39 / 789

33. $17.04 \mathrm{~g} ; 25.3 / 27.0 \mathrm{~mm} ; 7^{\text {h }}$ (MNM Budapest, inv. no. $14 / 1856.4)$

-, This coin: Dessewffy 1910, 12, no. 142, Tab. V: 142; Paulsen 1933, 114, no. 1921; FRMÖ I/2, 328, no. 5/4/38; Röttger 2019, Database no. 3-49 / 963

34. 17.02 g; $25.8 / 25.8 \mathrm{~mm}$; $6^{\text {h }}$ (MNM Budapest Coll. M. Dessewffy, inv. no. Dess. 142)

-, This coin: Fiala 1900, 201, no. 2823; Paulsen 1933, 114, no. 1923; FRMÖ I/2, 328, no. 5/4/35; Torbágyi 1992, no. 1; Röttger 2019, Database no. 3-51 / 1204.

35. $17.01 \mathrm{~g} ; 26.0 \mathrm{~mm} ; 6^{\mathrm{h}}$ (MNM Budapest - Coll. K. Niklovits, inv. no. N I 4530)

-, This coin: Paulsen 1933, 114, no. 1919 (without depiction); FRMÖ I/2, 328, no. 5/4/34; Röttger 2019, Database no. 3-74 / 902.

36. $16.58 \mathrm{~g} ; 24.1 / 25.4 \mathrm{~mm} ; 12^{\mathrm{h}}$ (MNM Budapest, Coll. Trau, inv. no. 6/1904.3)

\section{AR tetradrachm, Biatec group - BIATEC,} var. Röttger (2019) II/1 (1)B-1C

Obv.: male and female head to the right (Honos and Virtus), leaf (?) on the right, border of smooth line

Rev.: naked rider on horse to the right, rein in the left hand, in the right hand a branch terminating in pellets (mistletoe?), resting on his shoulder, right arm is bent between the horseman's chest and horse's neck, in exergue between two lines BIATEC, leaf before horse, border of smooth line and arches turned inwards

Ref.: Dessewffy 1910, Tab. V: 144; Paulsen 1933, Taf. 30: 723-730, 31: 731; Ondrouch 1958, nos. 31-34, 43, 47; Allen 1987, nos. 242, S235; Göbl 1994, Taf. 2: II/1 (1)-1:2-3; Dembski 1998, Taf. 30: 599-600; Kostial 2003, no. 63

This coin: Dessewffy 1910, 12, no. 144, Tab. V: 144; Paulsen 1933, 114, no. 1922; FRMÖ I/2, 328, no. 5/4/ 36; Röttger 2019, Database no. 4-13 / 964 


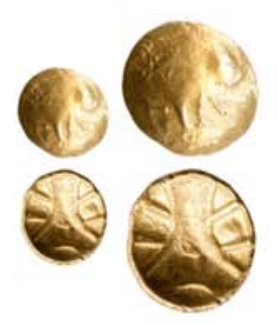

28

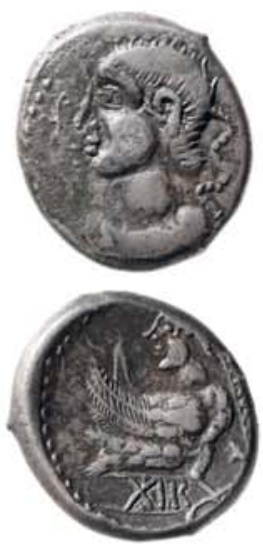

30
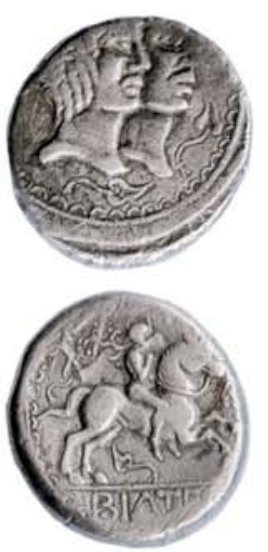

36
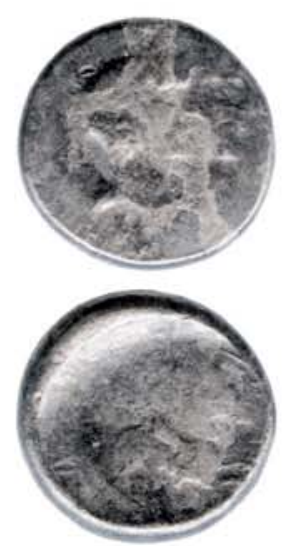

42
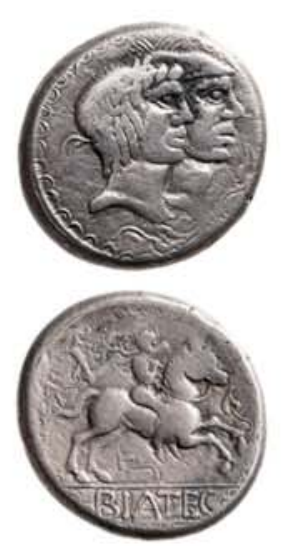

31
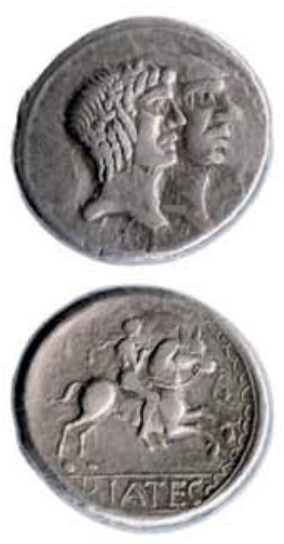

37
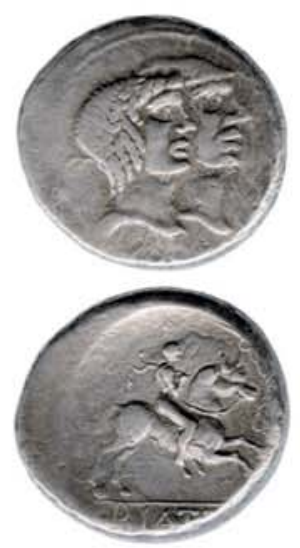

43
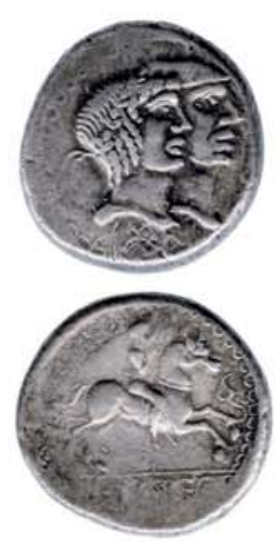

32
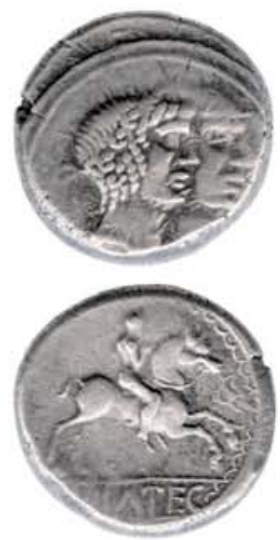

38
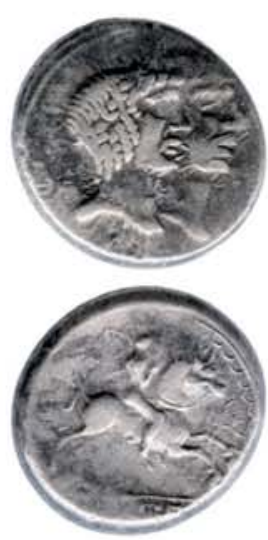

44

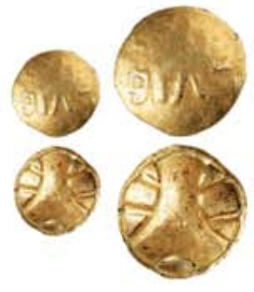

29
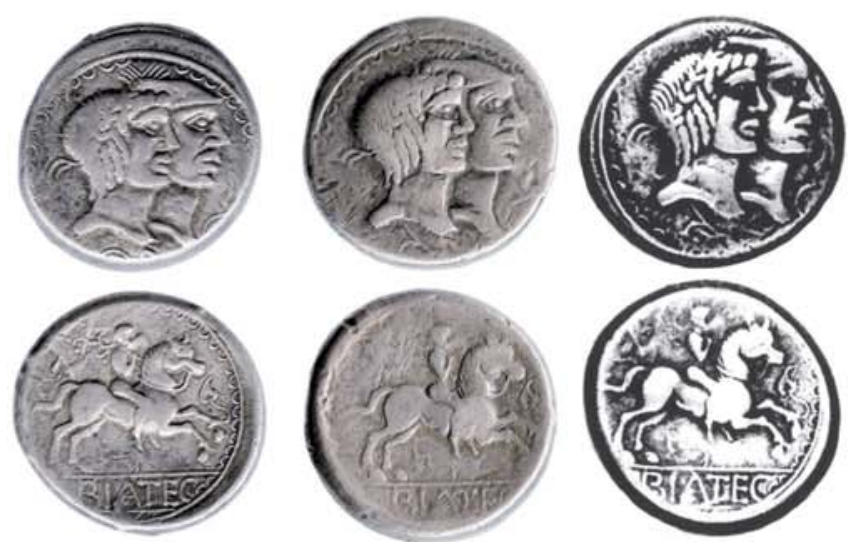

33
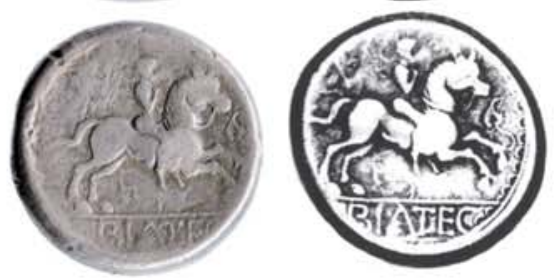

34

35
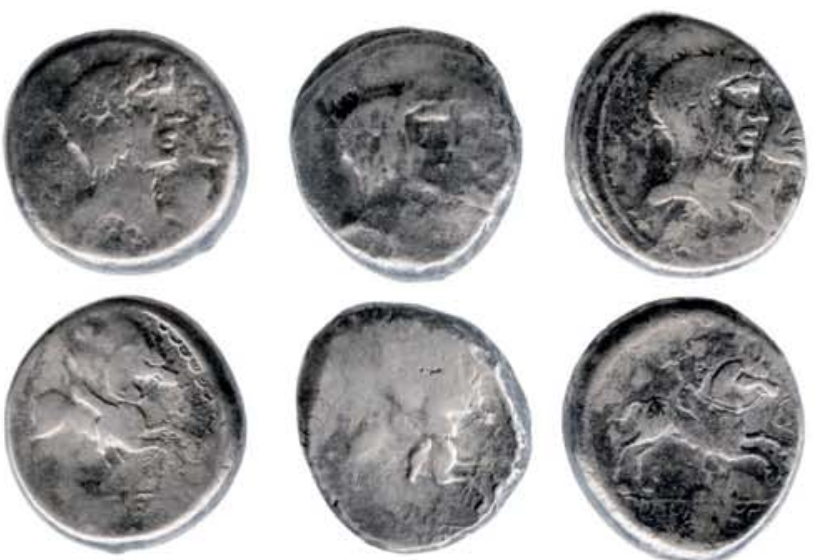

39

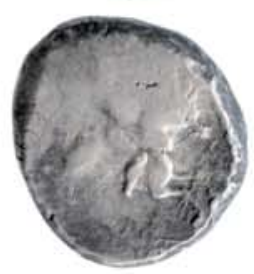

40

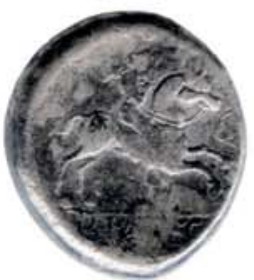

41
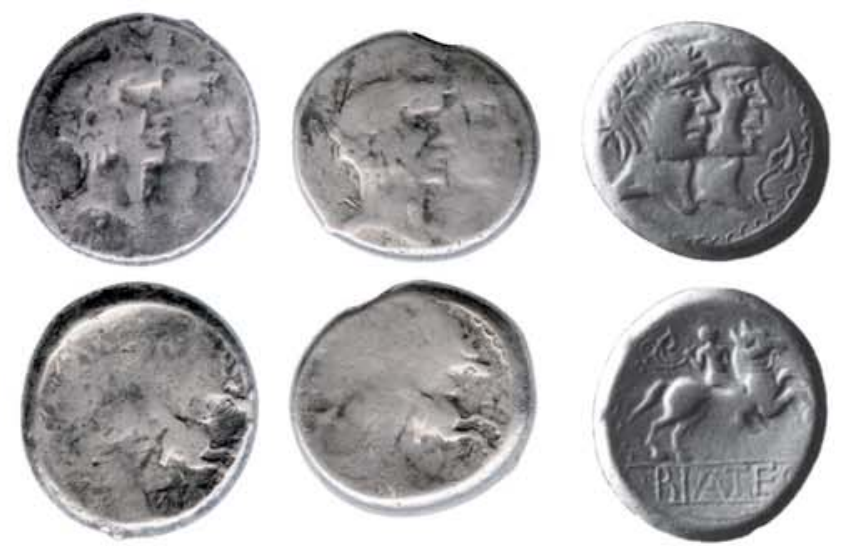

45

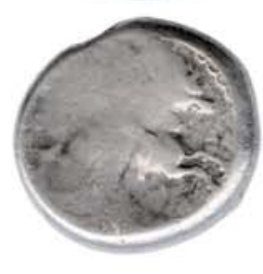

46

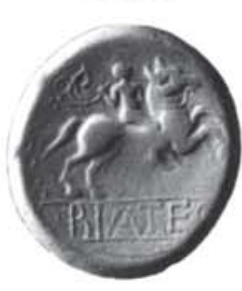

47

Fig. 4c. Deutsch Jahrndorf. Coins from the hoard (the numbers correspond to the catalogue; 28-29 - 1:1.5; 30-47 - 1:1; photos by J. Militky and KHM Wien). Obr. 4c. Deutsch Jahrndorf. Mince z pokladu (Čísla odpovídaji katalogu; 28-29-1: 1,5; 30-47-1: 1; foto J. Militký a KHM Wien). 
37. 17.22 g; $26.7 / 26.0 \mathrm{~mm} ; 6^{\mathrm{h}}$ (MNM Budapest Coll. M. Dessewffy, inv. no. Dess. 144)

-, This coin: Röttger 2019, Database no. 4-17 / 1213 38. $17.21 \mathrm{~g} ; 25.7 / 24.8 \mathrm{~mm} ; 12^{\mathrm{h}}$ (MNM Budapest, inv. no. $14 / 1856.22)$

-, This coin: Röttger 2019, Database no. 4-46 / 1214 39. $17.10 \mathrm{~g} ; 23.7 / 23.6 \mathrm{~mm}$; $1^{\mathrm{h}}$ (MNM Budapest, inv. no. $14 / 1856.25)$

-, This coin: Röttger 2019, Database no. 4-51 / 1217 40. $17.10 \mathrm{~g} ; 23.6 / 25.2 \mathrm{~mm} ; 7^{\text {h }}$ (MNM Budapest, inv. no. $14 / 1856.31)$

-, This coin: Paulsen 1933, 156, no. 725, Taf. 30: 725 (unprovenanced); Röttger 2019, Database no. 4-52 / 416 41. $17.07 \mathrm{~g} ; 24.3 / 27.0 \mathrm{~mm}$; $6^{\mathrm{h}}$ (MNM Budapest, inv. no. $14 / 1856.27)$

-, This coin: Röttger 2019, Database no. 4-56 / 1218 42. $17.06 \mathrm{~g} ; 25.9 / 25.2 \mathrm{~mm}$; $3^{\text {h }}$ (MNM Budapest, inv. no. $14 / 1856.29)$

-, This coin: Paulsen 1933, 114, no. 1923 (?); Röttger 2019, Database no. 4-64 / 1205

43. $17.06 \mathrm{~g} ; 27.2 / 25.5 \mathrm{~mm} ; 6^{\mathrm{h}}$ (MNM Budapest - Coll. K. Niklovits, inv. no. N I 4533)

-, This coin: Röttger 2019, Database no. 4-61 / 1212 44. $17.04 \mathrm{~g} ; 26.1 / 24.3 \mathrm{~mm}$; $6^{\mathrm{h}}$ (MNM Budapest, inv. no. $14 / 1856.24)$

-, This coin: Röttger 2019, Database no. 4-84 / 1215 45. $16.99 \mathrm{~g} ; 24.2 / 25.4 \mathrm{~mm} ; 12^{\mathrm{h}}$ (MNM Budapest, inv. no. $14 / 1856.30)$

-, This coin: Röttger 2019, Database no. 4-91 / 1216 46. $16.94 \mathrm{~g} ; 24.7 / 23.2 \mathrm{~mm}$; $12^{\mathrm{h}}$ (MNM Budapest, inv. no. $14 / 1856.32)$

AR tetradrachm, Biatec group - BIATEC, var. Göbl (1994) II/1 (2/1)-2

Obv.: male and female head to the right (Honos and Virtus), leaf right, border of smooth line and arches turned inwards

Rev.: naked rider on horse to the right, rein in the left hand, right arm lifted and pointing back, in right hand branch terminating in pellets (mistletoe?), in exergue between two lines BIATEC, border of smooth line and arches turned inwards

Ref.: Paulsen 1933, Taf. 31: 743-744; Ondrouch 1958, no. 122; Göbl 1994, Taf. 2: II/1 (2/1)-2; Dembski 1998, Taf. 30: 598; Kostial 2003, no. 65

This coin: Paulsen 1933, 115, 156, no. 744, Taf. 31: 744; FRMÖ I/2, 328, no. 5/4/43; Röttger 2019, Database no. 5-2 / 435

47. $17.30 \mathrm{~g} ; 25.7 \mathrm{~mm}$ (private collection, unknown present whereabouts)

-, This coin: Röttger 2019, Database no. 5-6 / 1219

48. $17.23 \mathrm{~g} ; 23.4 / 23.9 \mathrm{~mm} ; 3^{\text {h }}$ (MNM Budapest, inv. no. $14 / 1856.5)$
-, This coin: Paulsen 1933, 156, no. 743, Taf. 31: 743; FRMÖ I/2, 328, no. 5/4/42, Taf. 1: 42; Dembski 1998, 80, no. 598, Taf. 30: 598; Röttger 2019, Database no. $5-9 / 434$

49. $17.12 \mathrm{~g} ; 24.7 \mathrm{~mm} ; 7^{\mathrm{h}}$ (KHM Wien, inv. no. GR 27082)

AR tetradrachm, Biatec group - BIATEC, var. Göbl (1994) II/1 (2/2)-3

Obv.: male and female head to the right (Honos and Virtus), leaf right, border of smooth line and arches turned inwards

Rev.: naked rider on horse to the right, rein in the left hand, in the right hand a branch terminating in two leaves and three pellets (mistletoe?), in exergue between two lines BIATEC, border of smooth line and arches turned inwards

Ref.: Paulsen 1933, Taf. 31: 737-742; Ondrouch 1958, nos. 105, 107-109; Castelin 1978, nos. 1155-1156; Allen 1987, no. 243; Göbl 1994, Taf. 2: II/1 (2/2)-3; Kostial 2003, no. 66; Militký 2013b, no. 1

This coin: Röttger 2019, Database no. 6-2 / 1220

50. 17.42 g; $25.1 / 23.4 \mathrm{~mm} ; 4^{\mathrm{h}}$ (MNM Budapest, inv. no. $14 / 1856.13)$

-, This coin: Röttger 2019, Database no. 6-7 / 1222 51. 17.27 g; $25.6 / 24.8 \mathrm{~mm} ; 6^{\mathrm{h}}$ (MNM Budapest, inv. no. 14/1856.19)

-, This coin: Röttger 2019, Database no. 6-19 / 1224 52. $17.20 \mathrm{~g} ; 24.8 / 23.8 \mathrm{~mm} ; 7^{\mathrm{h}}$ (MNM Budapest, inv. no. $14 / 1856.11)$

-, This coin: Röttger 2019, Database no. 6-14 / 1223 53. $17.20 \mathrm{~g} ; 24.1 / 24.7 \mathrm{~mm} ; 6^{\mathrm{h}}$ (MNM Budapest, inv. no. 14/1856.10)

-, This coin: Röttger 2019, Database no. 6-20 / 1225 54. $17.19 \mathrm{~g} ; 26.0 / 24.7 \mathrm{~mm} ; 10^{\mathrm{h}}$ (MNM Budapest, inv. no. $14 / 1856.8$ )

-, This coin: Röttger 2019, Database no. 6-49 / 1221 55. $16.85 \mathrm{~g} ; 26.2 / 23.9 \mathrm{~mm}$; $9^{\text {h }}$; on both the av. and rv. secondary 'tremollo' linear cut (MNM Budapest, inv. no. 14/1856.6)

\section{AR tetradrachm, Biatec group - BIATEC, var. Göbl (1994) II/1 (3)-4}

Obv.: male and female head to the right (Honos and Virtus), leaf on the right, border of smooth line and arches turned inwards

Rev.: naked rider on horse to the right, rein in the left hand, in the right hand a branch terminating in three leaves, in exergue between two lines BIATEC, border of smooth line and arches turned inwards

Ref.: Paulsen 1933, Taf. 31: 732-736; Ondrouch 1958, nos. 67-70, 74-78; Göbl 1994, Taf. 2: II/1 (3)-4; Dembski 1998, Taf. 30: 594-596; Kostial 2003, no. 67; Ziegaus 2010, no. 485; Militký 2013b, no. 2

This coin: FRMÖ I/2, 328, no. 5/4/40, Taf. 1: 40; Göbl 1994, Taf. 7: T2; Dembski 1998, 80, no. 594, Taf. 30: 594; Röttger 2019, Database no. 7-8 / 761 


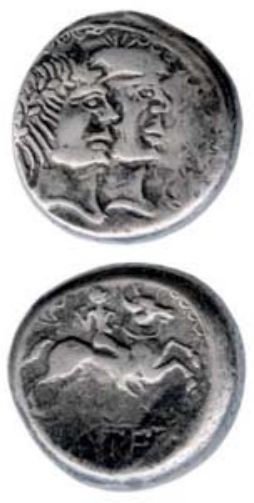

48

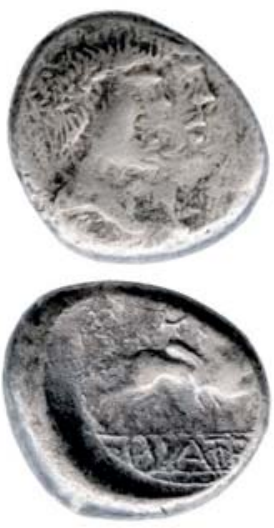

54
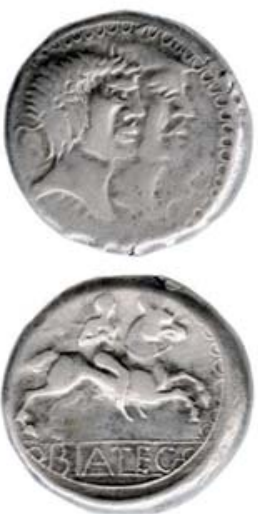

60

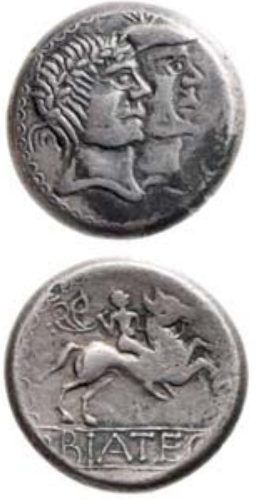

49

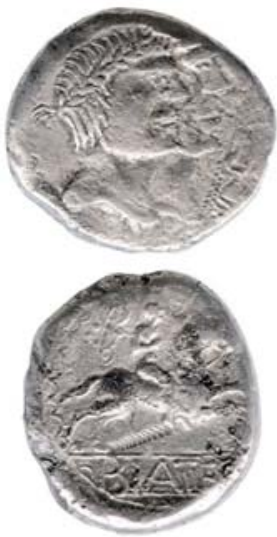

55
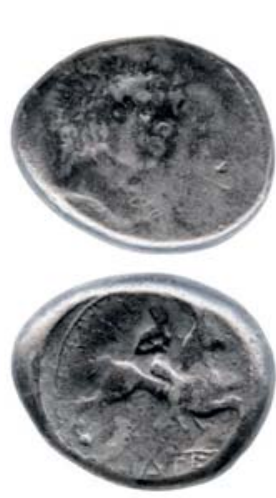

61
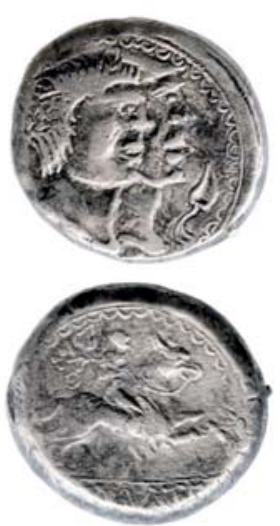

50
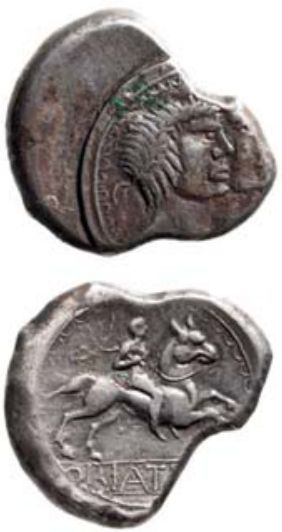

56

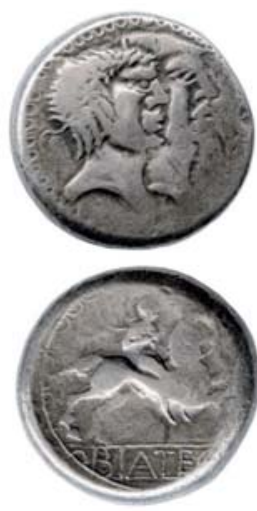

62
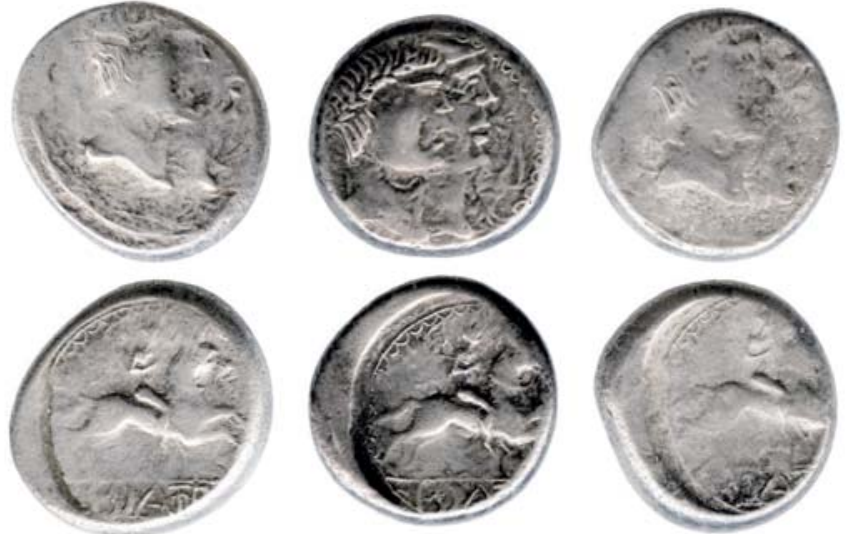

52

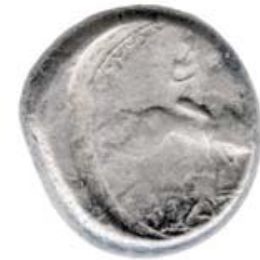

51
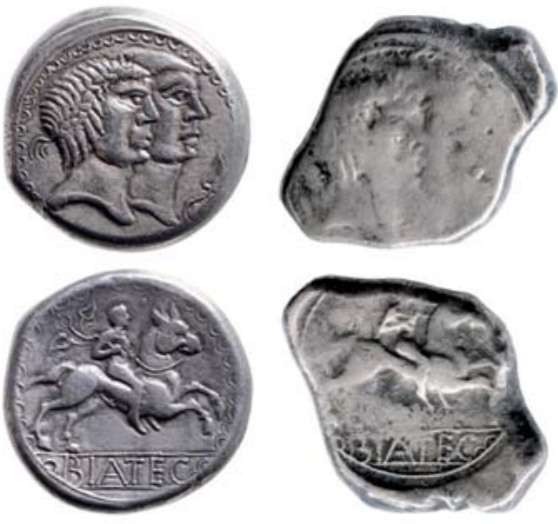

58

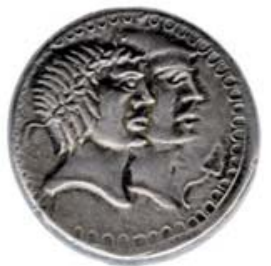

57
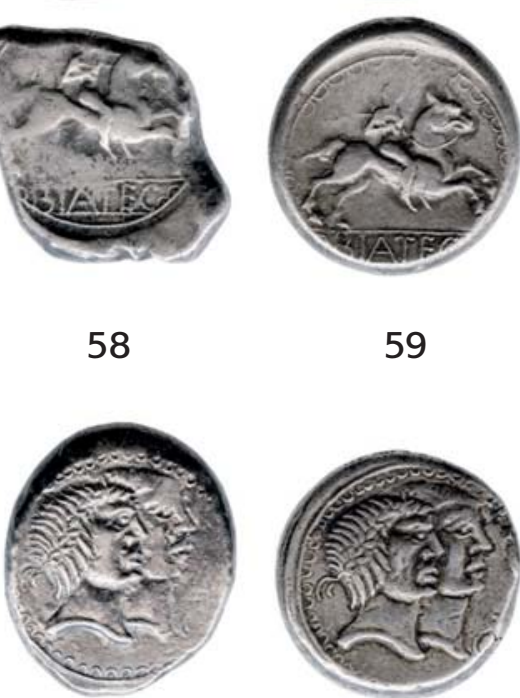

59
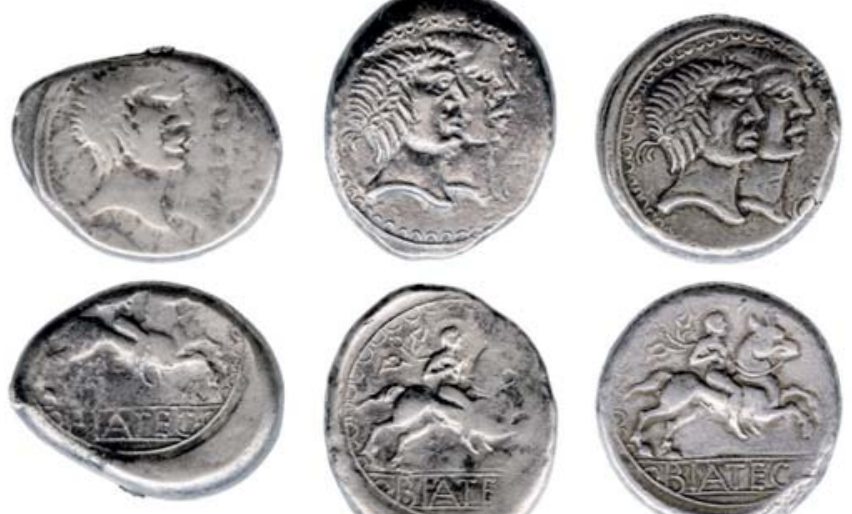

63

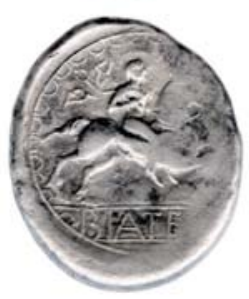

64

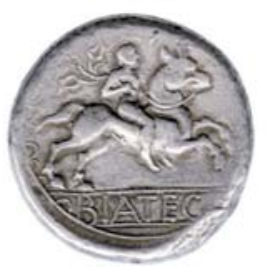

65

Fig. 4d. Deutsch Jahrndorf. Coins from the hoard (the numbers the correspond to catalogue; 1:1; photos by J. Militký and KHM Wien). - Obr. 4d. Deutsch Jahrndorf. Mince z pokladu (čísla odpovídaji katalogu; 7 : 1; foto J. Militký a KHM Wien).

56. $17.31 \mathrm{~g} ; 26.2 / 29.2 \mathrm{~mm} ; 6^{\text {h }}$ (KHM Wien, inv. no. GR 27083)

-, This coin: Paulsen 1933, 156, no. 732, Taf. 31: 732; FRMÖ I/2, 328, no. 5/4/39, Taf. 1: 39; Göbl 1994, Taf.
1: 4; Dembski 1998, 80, no. 595, Taf. 30: 595; Röttger 2019, Database no. 7-13 / 423

57. $17.27 \mathrm{~g} ; 24.9 / 24.6 \mathrm{~mm} ; 6^{\mathrm{h}}$ (KHM Wien, inv. no. GR 27087) 
-, This coin: Göbl 1994, Taf. 2: II/1(3)-4/3; Röttger 2019, Database no. 7-16 / 794

58. 17.26 g; $30.7 / 23.2 \mathrm{~mm} ; 6^{\mathrm{h}}$ (MNM Budapest, inv. no. $14 / 1856.18)$

-, This coin: Fiala 1900, 201, no. 2824, Taf. II: 2824; Paulsen 1933, 114, 156, no. 734, Taf. 31: 734; FRMÖ I/2, 328, no. 5/4/41; Röttger 2019, Database no. 7-15 / 425

59. 17.26 g; 25.8/26.5 mm; $6^{\text {h }}$ (MNM Budapest - Coll. Ernst Prinz zu Windisch-Grätz and K. Niklovits, inv. no. N I 4534)

-, This coin: Röttger 2019, Database no. 7-14 / 1211 60. $17.26 \mathrm{~g} ; 25.6 / 24.6 \mathrm{~mm} ; 6^{\mathrm{h}}$ (MNM Budapest, inv. no. $14 / 1856.33)$

-, This coin: Röttger 2019, Database no. 7-30 / 1263 61. $17.13 \mathrm{~g} ; 27.0 / 22.2 \mathrm{~mm} ; 6^{\mathrm{h}}$ (MNM Budapest, inv. no. $14 / 1856.16)$

-, This coin: Röttger 2019, Database no. 7-37 / 1191 62. $17.11 \mathrm{~g} ; 24.8 / 24.4 \mathrm{~mm}$; $6^{\text {h }}$ (MNM Budapest, inv. no. $14 / 1856.9)$

-, This coin: Röttger 2019, Database no. 7-36 / 1262 63. $17.11 \mathrm{~g} ; 27.9 / 22.2 \mathrm{~mm} ; 6^{\mathrm{h}}$ (MNM Budapest, inv. no. $14 / 1856.14)$

-, This coin: Paulsen 1933, 156, no. 735, Taf. 31: 735 (unprovenanced); Röttger 2019, Database no. 7-35 / 426

64. $17.09 \mathrm{~g} ; 23.8 / 28.1 \mathrm{~mm} ; 6^{\text {h }}$ (MNM Budapest, inv. no. $14 / 1856.20)$

-, This coin: Röttger 2019, Database no. 7-47 / 779 65. $17.04 \mathrm{~g} ; 25.1 / 25.4 \mathrm{~mm} ; 6^{\mathrm{h}}$ (MNM Budapest - Coll. K. Niklovits, inv. no. N I 4535)

-, This coin: Röttger 2019, Database no. 7-34 / 1260 66. $16.98 \mathrm{~g} ; 25.0 / 23.1 \mathrm{~mm} ; 12^{\mathrm{h}}$ (MNM Budapest, inv. no. $14 / 1856.15)$

-, This coin: Röttger 2019, Database no. 7-57 / 1261

67. $16.92 \mathrm{~g} ; 24.7 / 25.2 \mathrm{~mm} ; 6^{\text {h }}$ (MNM Budapest, inv. no. $14 / 1856.17)$

AR tetradrachm, Biatec group - BIATEC, var. Röttger (2019) II/2 (2)B-6Ba

Obv.: male and female head to the right (Honos and Virtus), on the right BIA, border of smooth line and arches turned inwards

Rev.: naked rider on horse to the right, rein in the left hand, in the right hand a branch terminating in three leaves, in exergue between two lines BIATEC, border of smooth line and arches turned inwards

Ref.: Kenner 1895, Taf. 1: 14; Paulsen 1933, Taf. 31: 745-747; Ondrouch 1958, nos. 123-126; Allen 1987, no. S236; Göbl 1994, Taf. 3: II/2 (2)-6/1; Dembski 1998, Taf. 30: 601-602

This coin: Kenner 1895, Taf. 1: 14; Paulsen 1933, 156, no. 745 , Taf. 31: 745; FRMÖ I/2, 328, no. 5/4/44, Taf. 1: 44; Göbl 1994, Taf. 3: II/2 (2)-6/1; Dembski 1998, 81, no. 601, Taf. 30: 601; Röttger 2019, Database no. $12-12 / 436$
68. $17.21 \mathrm{~g} ; 22.2 / 27.8 \mathrm{~mm} ; 7^{\text {h }}$ (KHM Wien, inv. no. GR 27084)

-, This coin: Paulsen 1933, 115, 156, no. 747 , Taf. 31 : 747; Röttger 2019, Database no. 12-28 / 438

69. $17.10 \mathrm{~g} ; 28.2 \mathrm{~mm}$ (private collection, unknown present whereabouts)

-, This coin: Röttger 2019, Database no. 12-54 / 1264 70. $16.83 \mathrm{~g} ; 26.9 / 21.9 \mathrm{~mm} ; 1^{\mathrm{h}}$ (MNM Budapest, inv. no. $14 / 1856.21)$

AR tetradrachm, Biatec group - BIATEC, var. Röttger (2019) II/2 (2)B-6Bb

Obv.: male and female head to the right (Honos and Virtus), on the right BIA (reworked letter B), border of smooth line and arches turned inwards

Rev.: naked rider on horse to the right, rein in the left hand, in the right hand a branch terminating in three leaves, in exergue between two lines BIATEC, border of smooth line and arches turned inwards

Ref.: Paulsen 1933, Taf. 31: 748; Göbl 1994, Taf. 3: II/2

(2)-6/2; Kostial 2003, nos. 68-69

This coin: Röttger 2019, Database no. 12-34 / 1265

71. 17.06 g; $26.7 / 24.8 \mathrm{~mm}$; $9^{\text {h }}$ (MNM Budapest, inv. no. $14 / 1856.23)$

-, This coin: Göbl 1994, Taf. 3: II/2(2)-6/2; Röttger 2019, Database no. 12-44 / 795

72. $16.97 \mathrm{~g} ; 26.4 / 24.8 \mathrm{~mm}$; $9^{\text {h }}$ (MNM Budapest - Coll. K. Niklovits, inv. no. N I 4537)

\section{AR tetradrachm, Biatec group - BIATEC, var. Göbl (1994) II/3-12}

Obv.: male and female head to the right (Honos and Virtus), marked border of dots

Rev.: rider with naked (?) torso and trousers on saddled horse to the right, holding sword in right hand and large oval shield (oval line around horseman's upper body and horse's head) and rein (?) in left hand, saddle straps visible on horse's body, BIATEC in exergue

Ref.: Paulsen 1933, Taf. 32: 752-754; Ondrouch 1958, nos. 128-134; Allen 1987, no. 241; Göbl 1994, Taf. 3: II/3-12; Dembski 1998, Taf. 30: 603-604, 31: 605-606; Kostial 2003, no. 70; Militký 2013b, no. 3

This coin: FRMÖ I/2, 328, no. 5/4/45, Taf. 1: 45; Dembski 1998, 81, no. 605, Taf. 31: 605; Röttger 2019, Database no. 13-23 / 765

73. $17.12 \mathrm{~g} ; 25.4 \mathrm{~mm} ; 11^{\mathrm{h}}$ (KHM Wien, inv. no. GR 27086)

-, This coin: Röttger 2019, Database no. 13-35 / 1266 74. $17.05 \mathrm{~g} ; 24.7 / 23.2 \mathrm{~mm} ; 12^{\mathrm{h}}$ (MNM Budapest, inv. no. $14 / 1856.34)$

\section{AR tetradrachm, Biatec group - BIATEC, var. Göbl (1994) II/4-3}

Obv.: male head with short hair to right, border of double smooth line and arches turned inwards

Rev.: naked rider on horse to the right, rein in left hand, in right hand branch terminating in two leaves and three pellets (mistletoe?), in exergue between two lines BIATEC, border of smooth line and arches turned inwards 


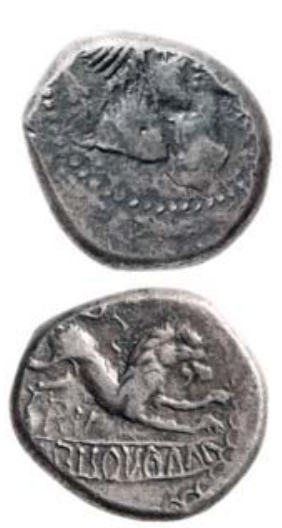

87
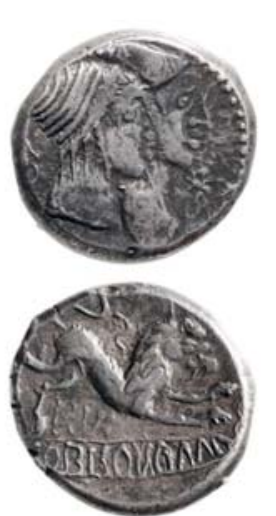

88
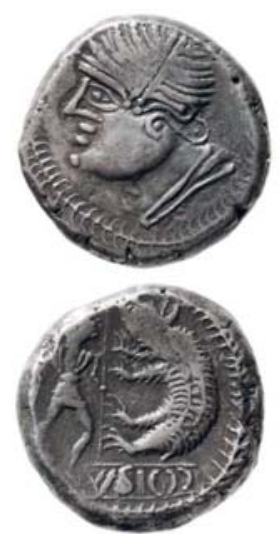

89
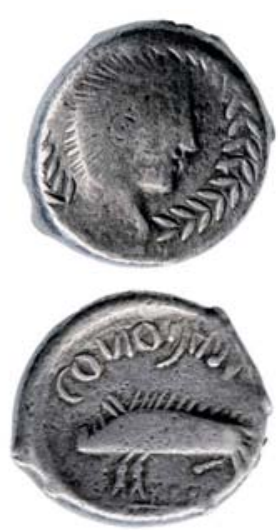

90
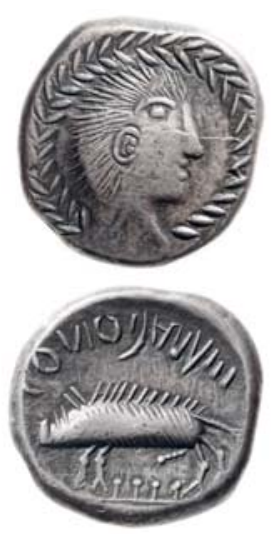

91
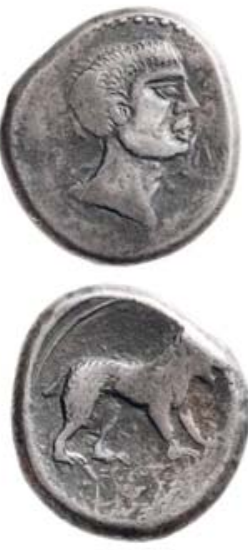

98
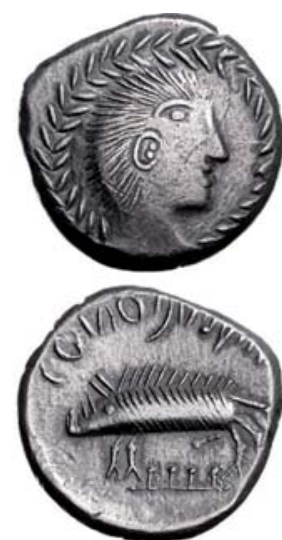

92

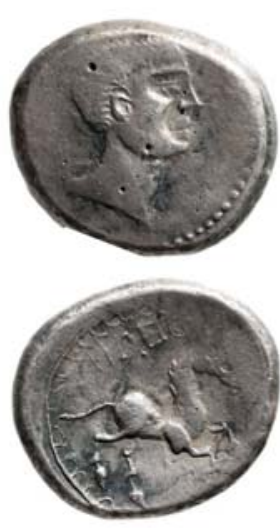

93

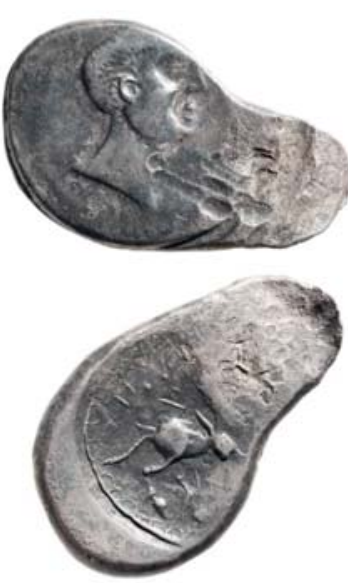

95
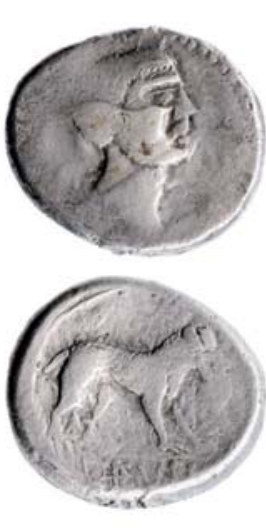

96
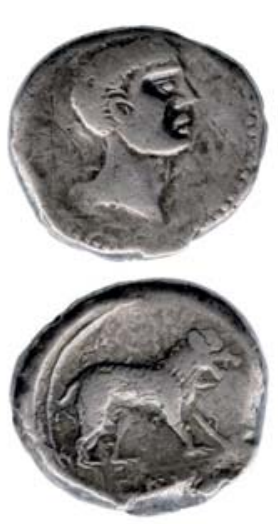

97
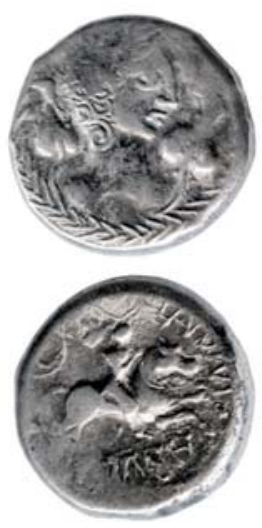

102
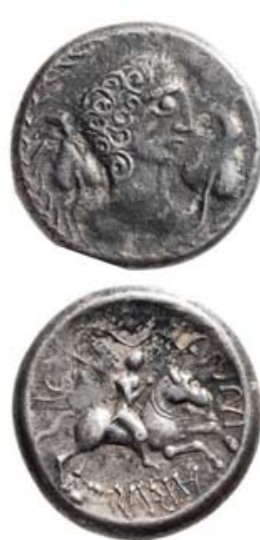

103
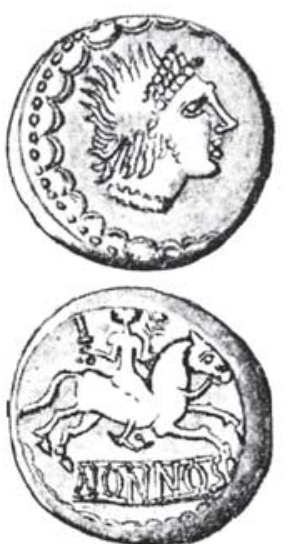

104
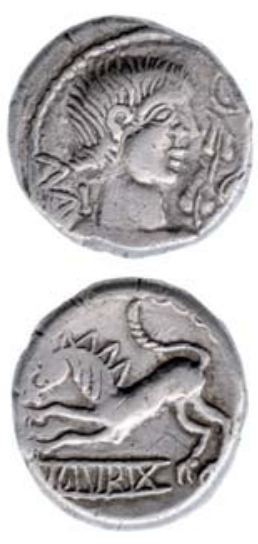

99

Fig. 4f. Deutsch Jahrndorf. Coins from the hoard (the numbers the correspond to catalogue; 1:1; photos by J. Militky and KHM Wien). - Obr. 4f. Deutsch Jahrndorf. Mince z pokladu (Čisla odpovídaji katalogu; 1 : 1; foto J. Militký a KHM Wien).

Ref.: Paulsen 1933, Taf. 30: 717; Ondrouch 1958, no. 6; Göbl 1994, Taf. 3: II/4-3; Militký 2013b, no. 4

This coin: Göbl 1994, Taf. 3: II/4-3/1; Röttger 2019, Database no. 14-15/797

75. 17.02 g; 22.3/30.3 mm; 9h (MNM Budapest - Coll. K. Niklovits, Inv. no. N I 4528)
AR tetradrachm, Biatec group - BIATEC, var. Göbl (1994) II/4-7

Obv.: male head with short hair to right, border of double smooth line and arches turned inwards

Rev.: naked rider on horse to the right, rein in left hand, in right hand a branch terminating in three leaves, in 
exergue between two lines BIATEC, border of smooth line and arches turned inwards

Ref.: Paulsen 1933, Taf. 30: 711-716; Ondrouch 1958, nos. 1-5, 7-24; Allen 1987, no. 244, S237; Göbl 1994, Taf. 3: II/4-7; Kostial 2003, no. 71; Ziegaus 2010, no. 486; Militký 2013b, no. 5

This coin: Paulsen 1933, 115, 156, no. 1931 (not depicted); FRMÖ I/2, 327, no. 5/4/31; Röttger 2019, Database no. 15-6 / 1208

76. $17.30 \mathrm{~g} ; 27.5 \mathrm{~mm}$ (private collection, unknown present whereabouts)

-, This coin: Röttger 2019, Database no. 15-20 / 402

77. $17.15 \mathrm{~g} ; 26.2 / 23.2 \mathrm{~mm} ; 7^{\text {h }}$ (MNM Budapest, inv. no. $14 / 1856.3)$

-, This coin: Röttger 2019, Database no. 15-26 / 1538 78. 17.13 g; $25.0 / 25.9 \mathrm{~mm} ; 6^{\mathrm{h}}$ (MNM Budapest - Coll. K. Niklovits, inv. no. N I 4529)

-, This coin: Röttger 2019, Database no. 15-31 / 1267 79. $17.09 \mathrm{~g} ; 28.5 / 26.2 \mathrm{~mm}$; $6^{\mathrm{h}}$ (MNM Budapest, inv. no. $14 / 1856.1)$

AR tetradrachm, Biatec group - BVSSVMARVS, var. Göbl (1994) III/1-24

Obv.: male (?) bust to left, hair attached on nape, on left S-shaped branch (or feather?), marked border of dots Rev.: gryphon prancing to the right, below in rectangular frame BVSSVM, on right A-RV-S, border of smooth line and arches turned inwards

Ref.: Kupido 1866, Taf. 4: 4; Paulsen 1933, Taf. 33: 790, 34: 791; Ondrouch 1958, no. 239; Göbl 1994, Taf. 3: III/1-24; Dembski 1998, Taf. 33: 630-631

This coin: Paulsen 1933, 158, no. 791, Taf. 34: 791; FRMÖ I/2, 329, no. 5/4/69, Taf. 2: 69; Dembski 1998, 82, no. 630, Taf. 33: 630; Röttger 2019, Database no. $18-1 / 481$

80. $17.15 \mathrm{~g} ; 25.9 \mathrm{~mm} ; 9^{\mathrm{h}}$ (KHM Wien, inv. no. GR 27104)

-, This coin: Kupido 1866, Taf. 4: 4; Paulsen 1933, 158, no. 790, Taf. 33: 790; FRMÖ I/2, 329, no. 5/4/68, Taf. 2: 68; Göbl 1994, Taf. 3: III/1-24/1; Dembski 1998, 82, no. 631, Taf. 33: 631; Röttger 2019, Database no. 18-3 / 480

81. 17.09 g; $25.8 / 28.4 \mathrm{~mm}$; $3^{\text {h }}$ (KHM Wien, inv. no. GR 27099)

AR tetradrachm, Biatec group - BVSV, var. Göbl (1994) IV/1-28

Obv.: male (?) bust to left, hair attached on nape, on left S-shaped branch (or feather?), marked border of dots

Rev.: winged centaur (?) with female semi-figure to left looking back, raised tail, below between legs BVSV, marked border of dots

Ref.: Dessewffy 1910, Tab. V: 150; Paulsen 1933, Taf. 33: 785-789; Ondrouch 1958, nos. 232-238; Castelin 1978, no. 1161; Allen 1987, no. 249; Göbl 1994, Taf. 4: IV/1-28; Dembski 1998, Taf. 32: 627-628, 33: 629; Kostial 2003, nos. 72-73; Ziegaus 2010, nos. 491; Militký $2013 b$, no. 6

This coin: Egger 1898, no. 9 (without photograph); Röttger 2019, Database no. 19-61 / 1875

Comment: Provenance from the Deutsch Jahrndorf hoard is mentioned in the catalogue Egger 1898 as Német-Ujvár.

82. 17.1 g; 26 mm (Coll. Carl Leopold Latour v. Thurmburg, unknown present whereabouts)

-, This coin: FRMÖ I/2, 329, no. 5/4/66, Taf. 2: 66; Dembski 1998, 82, no. 627, Taf. 32: 627; Röttger 2019, Database no. 19-15 / 772

83. $17.07 \mathrm{~g} ; 22.7 / 26.7 \mathrm{~mm} ; 10^{\mathrm{h}}$ (KHM Wien, inv. no. GR 27103)

-, This coin: Egger 7, no. 720 (with photograph); Desseuffy 1910, 12, no. 150, Tab. V: 150; Röttger 2019, Database no. 19-25 / 970

Comment: Provenance from the Deutsch Jahrndorf hoard is mentioned in the catalogue Egger 7. According to Dessewffy (1910) the find-spot is (probably incorrectly) Reca.

84. $17.00 \mathrm{~g} ; 24.0 / 25.0 \mathrm{~mm} ; 10^{\mathrm{h}}$ (MNM Budapest Coll. M. Dessewffy, inv. č. Dess. 150)

-, This coin: Kerényi 1959, Taf. 3: 10; Röttger 2019, Database no. 19-28 / 1268

85. $16.98 \mathrm{~g} ; 27.4 / 25.5 \mathrm{~mm} ; 7^{\mathrm{h}}$ (MNM Budapest, inv. no. $14 / 1856.74)$

-, This coin: Fiala 1900, 202, no. 2830; Paulsen 1933, 115, no. 2025; FRMÖ I/2, 329, no. 5/4/67; Röttger 2019, Database no. 19-51 / 1024.

86. $16.28 \mathrm{~g} ; 25 \mathrm{~mm}$ (Coll. Ernst Prinz zu WindischGrätz, unknown present whereabouts)

\section{AR tetradrachm, Biatec group - COBROVOMARVS, var. Göbl (1994) V/1-18}

Obv.: male and female head to right (Honos and Virtus or Dioscuri), leaf on left, star on right, marked border of dots

Rev.: lion prancing to right, in exergue between two lines COBROVOMA, below the lion stomach RV, above 2 , border of smooth line and arches turned inwards Ref.: Paulsen 1933, Taf. 34: 795-797; Ondrouch 1958, no. 244; Göbl 1994, Taf. 4: V/1-18; Dembski 1998, Taf. 33: 636-639; Kostial 2003, no. 74; Ziegaus 2010, no. 487; Militký 2013b, no. 7

This coin: FRMÖI/2, 329, no. 5/4/73, Taf. 2: 73; Dembski 1998, 82, no. 637, Taf. 33: 637; Röttger 2019, Database no. 20-5 / 775

87. $17.27 \mathrm{~g} ; 26.1 \mathrm{~mm} ; 4^{\text {h }}$ (KHM Wien, inv. no. GR 27097)

-, This coin: Kupido 1866, Taf. 4: 1; Paulsen 1933, 158, no. 796, Taf. 34: 796; FRMÖ I/2, 329, no. 5/4/72, Taf. 2: 72; Dembski 1998, 82, no. 638, Taf. 33: 638; Röttger 2019, Database no. 20-9 / 486

88. $17.11 \mathrm{~g} ; 25.2 \mathrm{~mm} ; 12^{\mathrm{h}}$ (KHM Wien, inv. no. GR 27098)

\section{AR tetradrachm, Biatec group - COISA, var. Göbl (1994) VI/1-21}

Obv.: male head in helmet (or hat) to left, hint of drape at the neck, border of 'chain' line

Rev.: standing male in short garment to right fighting rampant bear (?) or boar (?) to left, below in exergue between two lines COISA (inscription turned by $180^{\circ}$ ), border of 'chain' line (hardly visible) 


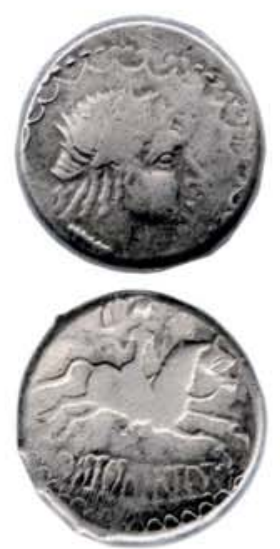

106

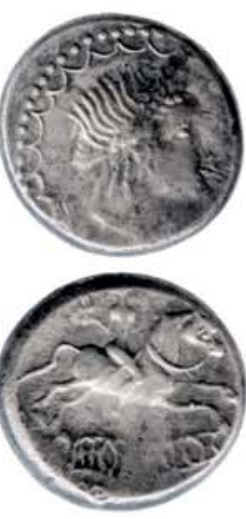

112
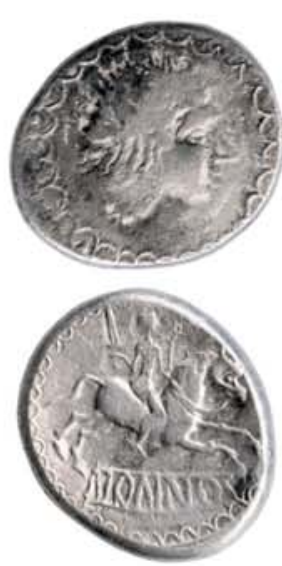

118

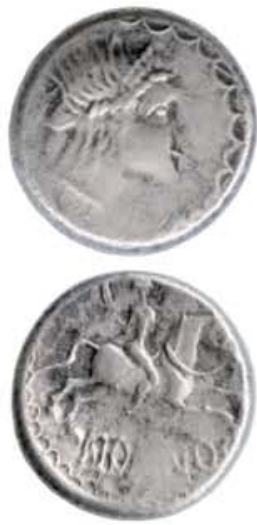

107
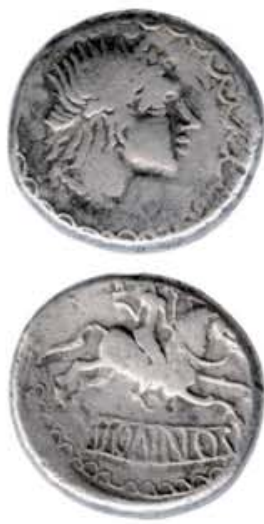

113
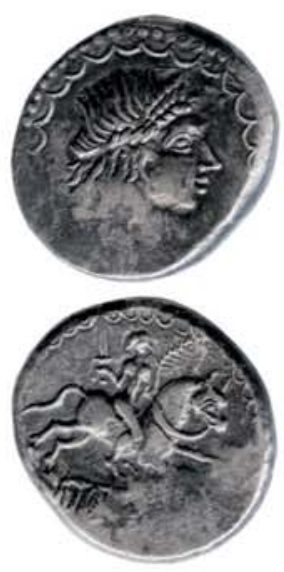

119

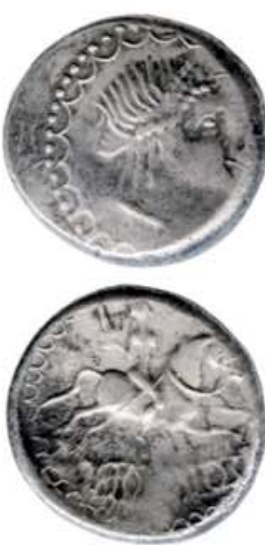

108
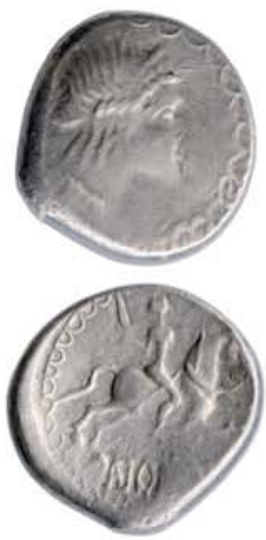

114
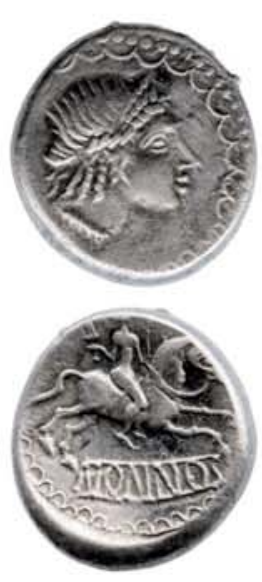

120
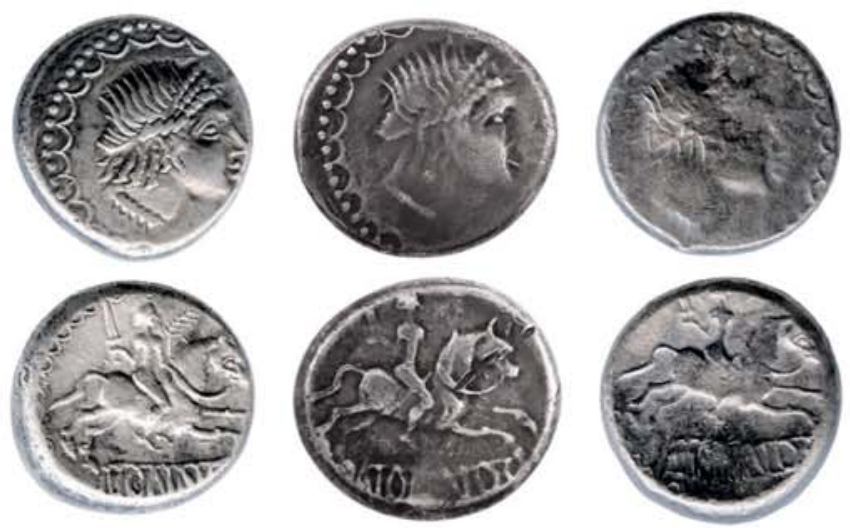

109
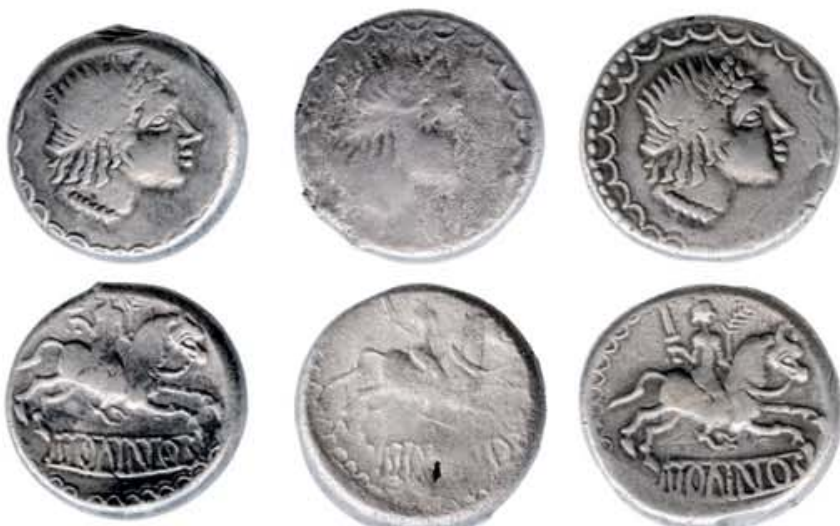

115

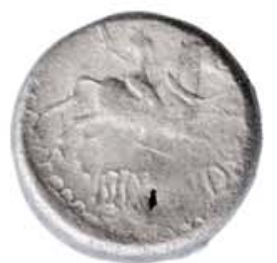

116

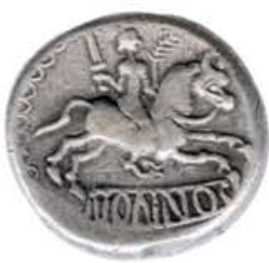

117
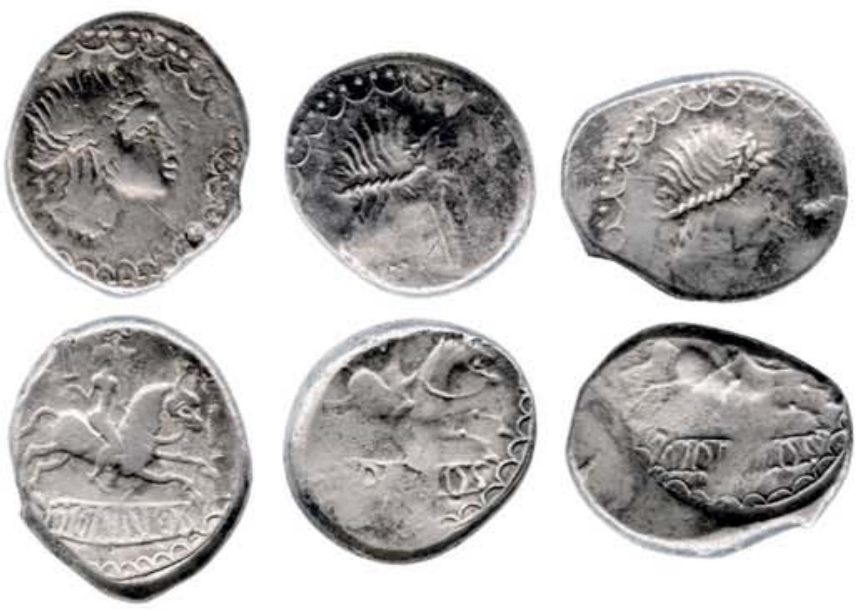

121

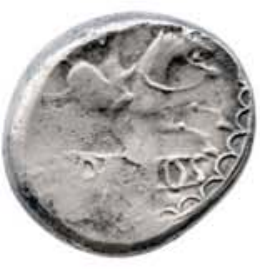

122

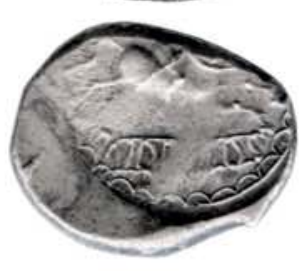

123

Fig. 4g. Deutsch Jahrndorf. Coins from the hoard (the numbers the correspond to catalogue; 1:1; photos by J. Militký and KHM Wien). - Obr. 4g. Deutsch Jahrndorf. Mince z pokladu (čisla odpovídaji katalogu; 1 : 1; foto J. Militký a KHM Wien).

Ref.: Kupido 1866, Taf. 4: 3; Paulsen 1933, Taf. 34: 798801; Ondrouch 1958, no. 245; Göbl 1994, Taf. 4: VI/121; Dembski 1998, Taf. 33: 640, 34: 641; Militký 2013b, nos. $8-10$
This coin: Kupido 1866, Taf. 4: 3; Paulsen 1933, 88, 158, no. 798, Taf. 34: 798; Pink 1974, Taf. VI: 85; FRMÖ I/2, 329, no. 5/4/74, Taf. 2: 74; Göbl 1994, Taf. 4: VI/1-21/1; Dembski 1998, 82, no. 640, Taf. 33: 640; 
Röttger 2019, Database no. 21-4 / 488; Militký 2020, 314-315, Abb. 9.9: 12

89. $17.17 \mathrm{~g} ; 25.6 \mathrm{~mm} ; 11^{\mathrm{h}}$ (KHM Wien, inv. no. GR 27095)

AR tetradrachm, Biatec group - COVIOMARVS, var. Göbl (1994) VII/1-23

Obv.: youthful male head to right, border of 'spruce' wreath

Rev.: stylised boar to left, below straight line with four stylised plants (?), above COVIOMARVS, border of smooth line

Ref.: Kupido 1866, Taf. 4: 2; Paulsen 1933, Taf. 35: 822825; Göbl 1994, Taf. 4: VII/1-23; Dembski 1998, Taf. 34: 647

This coin: Fiala 1900, 202, no. 2829; Paulsen 1933 , 115, 158, no. 823, Taf. 35: 823; Ondrouch 1961, X: 11; Pink 1974, Taf. VI: 87; FRMÖ I/2, 329, no. 5/4/77; Röttger 2019, Database no. 22-1 / 513

90. 17.50 g; $24.2 / 27.0$ mm; $9^{\text {h }}$ (MNM Budapest - Coll. Ernst Prinz zu Windisch-Grätz and K. Niklovits, inv. no. N I 4561)

-, This coin: Kupido 1866, Taf. 4: 2; Pink 1932, Taf. I: 5; Paulsen 1933, 93, 158, no. 822, Taf. 35: 822; FRMÖ I/2, 329, no. 5/4/76, Taf. 2: 76; Kolníková 1991, 86, Fig. 32-33; Göbl 1994, Taf. 4: VII/1-23/1; Dembski 1998, 83, no. 647, Taf. 34: 647; Röttger 2019, Database no. 22-2 / 512; Militký 2020, 314-315, Abb. 9.9: 13

91. $17.08 \mathrm{~g} ; 26.4 \mathrm{~mm}$; $9^{\text {h }}$ (KHM Wien, inv. no. GR 27096)

-, This coin: Egger 7, no. 719 (with depiction); Paulsen 1933, 158, no. 824, Taf. 35: 824 (unprovenanced); Ondrouch 1958, Tab. 23: 1 (unprovenanced); Röttger 2019, Database no. 22-5 / 514

Comment: provenance of the coin from the hoard is mentioned in the catalogue Egger 7; the coin was acquired for the Berlin collection from Artur Löbecke in 1906

92. 16.79 g; 26 mm (Münzkabinet Berlin, inv. no. 18204966) ${ }^{15}$

\section{AR tetradrachm, Biatec group - DEVIL (or DEVII), ${ }^{16}$ var. Göbl (1994) IX/1-16}

Obv.: male head with short hair to right, border of dots Rev.: horse prancing to right, above DEVIL or DEVII (legend is turned by $180^{\circ}$ ), border of smooth line and arches turned inwards

Ref.: Paulsen 1933, Taf. 33: 779-781; Ondrouch 1958, nos. 216-225; Allen 1987, no. 248, S240; Göbl 1994, Taf. 4: IX/1-16; Dembski 1998, Taf. 32: 621-623; Militký 2013b, no. 11

This coin: Paulsen 1933, 114, no. 1915 (without depiction); FRMÖ I/2, 329, no. 5/4/60, Taf. 2: 60; Dembski 1998, 81, no. 622, Taf. 32: 622; Röttger 2019, Database no. $24-4$ / 769

93. $17.26 \mathrm{~g} ; 27.5 / 23.6 \mathrm{~mm} ; 12^{\mathrm{h}}$ (KHM Wien, inv. no. GR 27081)

\footnotetext{
15 https://ikmk.smb.museum/object?id $=18204966$

16 An alternative reading proposed for the legend DEVIL is DEVII (Röttger 2013, 34-36), see chapter 5.2.
}

-, This coin: Paulsen 1933, 114, no. 1920 (without depiction); FRMÖ I/2, 329, no. 5/4/62; Röttger 2019, Database no. 471 (by mistake associated with the coin find Paulsen / 1933/, Taf. 33: 781)

Comment: provenance from the hoard is not certain 94. $17.07 \mathrm{~g} ; 26.3 \mathrm{~mm}$ (MNM Budapest - not located at present)

-, This coin: FRMÖ I/2, 329, no. 5/4/61, Taf. 2: 61; Göbl 1994, Taf. 7: T11; Dembski 1998, 81, no. 623, Taf. 32: 623; Röttger 2019, Database no. 24-21 / 770

95. $17.01 \mathrm{~g} ; 36.7 / 22.5 \mathrm{~mm} ; 6^{\text {h }}$ (KHM Wien, inv. no. GR 27089)

AR tetradrachm, Biatec group - DEVIL (or DEVII), var. Göbl (1994) IX/1-20

Obv.: male head with short hair to right, border of dots Rev.: standing bear to right, below in exergue between two lines DEVIL or DEVII, border of smooth line Ref.: Paulsen 1933, Taf. 33: 782-784; Ondrouch 1958, nos. 226-231; Castelin 1978, no. 1160; Göbl 1994, Taf. 4: IX/1-20; Dembski 1998, Taf. 32: 624-626; Kostial 2003, no. 76; Militký 2013b, nos. 12-13

This coin: Paulsen 1933, 114, 157, no. 782 , Taf. 32: 782 (provenance from the hoard is not certain); FRMÖ I/2, 329, no. 5/4/63; Röttger 2019, Database no. 25-16 / 472 Comment: provenance from the hoard is not certain 96. $17.07 \mathrm{~g} ; 26.7 / 23.6 \mathrm{~mm} ; 12^{\mathrm{h}}$ (MNM Budapest - Coll. Áldor, inv. no. 95/1907.2)

-, This coin: Paulsen 1933, 115, no. 1930? (but 16.96 g); FRMÖ I/2, 329, no. 5/4/65; Röttger 2019, Database no. 25-15 / 1025

Comment: provenance of this coin from the hoard is not certain - according to Ch. Röttger (2019) the find-spot is Simmeringer Heide

97. 17.07 g; 26.5/24.3 mm; $12^{\mathrm{h}}$ (MNM Budapest - Coll. Ernst Prinz zu Windisch-Grätz (?) and K. Niklovits, inv. no. N I 4556)

-, This coin: Paulsen 1933, 157, no. 783, Taf. 33: 783; FRMÖ I/2, 329, no. 5/4/64, Taf. 2: 64; Dembski 1998, 81, no. 625, Taf. 32: 625; Röttger 2019, Database no. 25-21 / 473

98. $17.00 \mathrm{~g} ; 26.5 \mathrm{~mm} ; 12^{\mathrm{h}}$ (KHM Wien, inv. no. GR 27090)

AR tetradrachm, Biatec group - EVOIVRIX (or FAPIARIX), ${ }^{17}$ var. Göbl (1994) X/2-19

Obv.: youthful male bust to right, on right four leaves, on left monogram $A \bar{A}$

Rev.: lion prancing to left with stylised mane of triangles, below between two lines EVOIVRIX, border of smooth line and arches turned inwards

Ref.: Paulsen 1933, Taf. 35: 817-819; Göbl 1994, Taf. 4: X/2-19; Dembski 1998, Taf. 34: 646; Kostial 2003, no. 77; Ziegaus 2010 , no. 487

This coin: Paulsen 1933, 158, no. 818, Taf. 35: 818 (unprovenanced); Röttger 2019, Database no. 27-12 / 508 99. $16.54 \mathrm{~g} ; 25.4 / 24.7 \mathrm{~mm} ; 7^{\mathrm{h}}$ (MNM Budapest, inv. no. $14 / 1856.71)$

\footnotetext{
17 Ch. Röttger (2013, 37-38) proposed to read the legend as mirrored: FAPIARIX. Cf. chapter 5.2.
} 


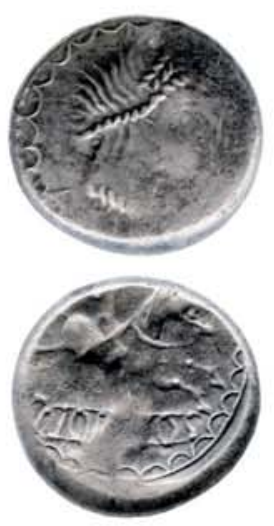

124
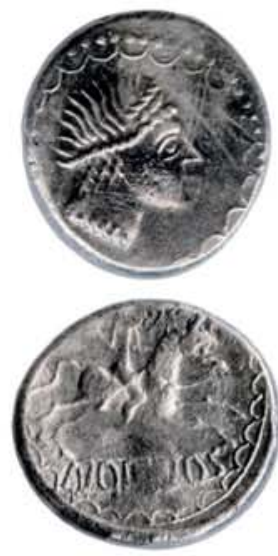

130

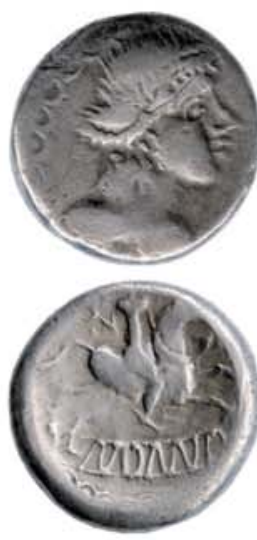

136
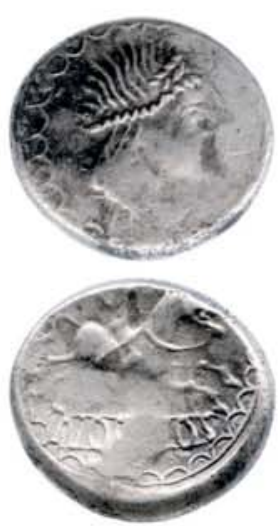

125
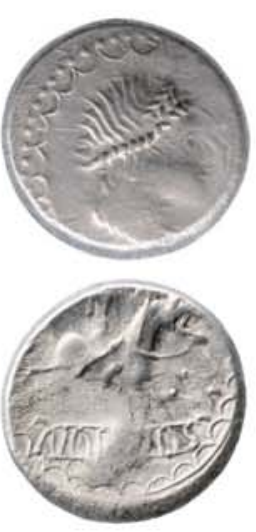

131
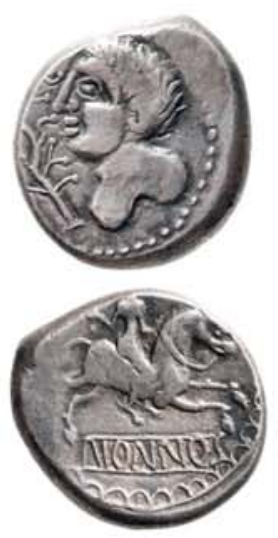

137
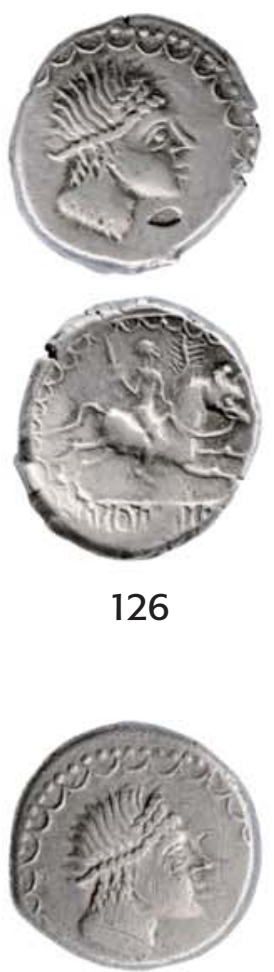

126

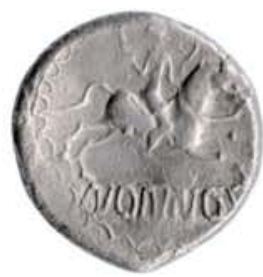

132
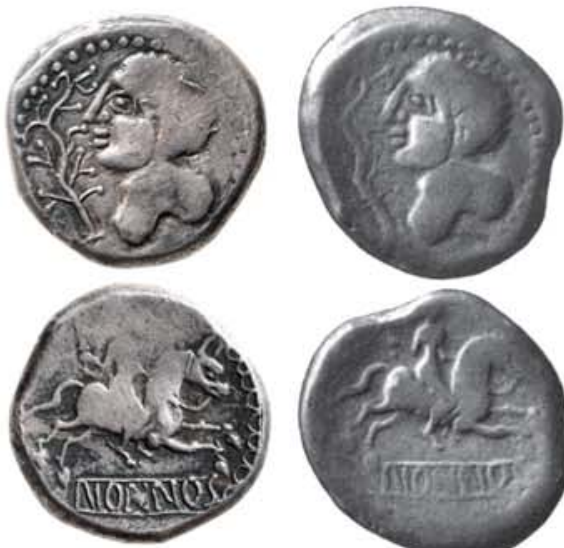

138

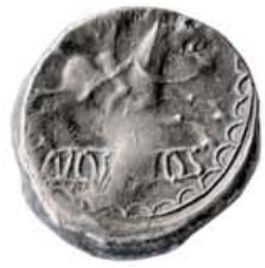

127
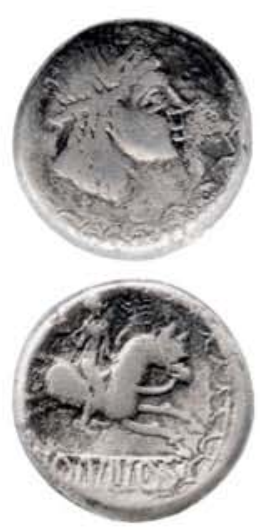

133

139
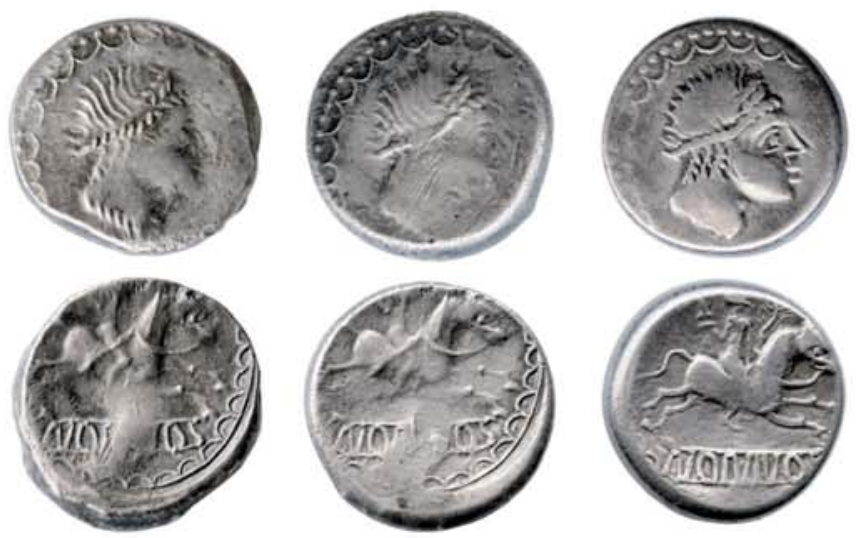

128

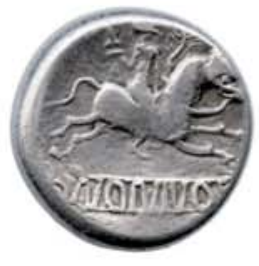

129
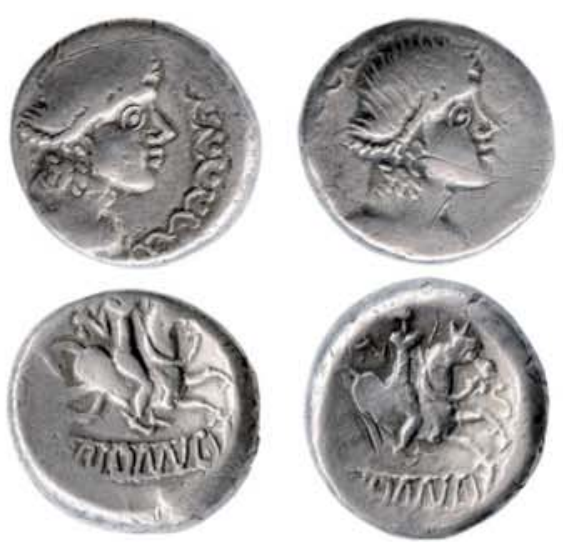

134

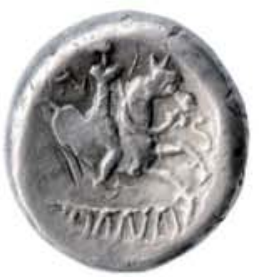

135
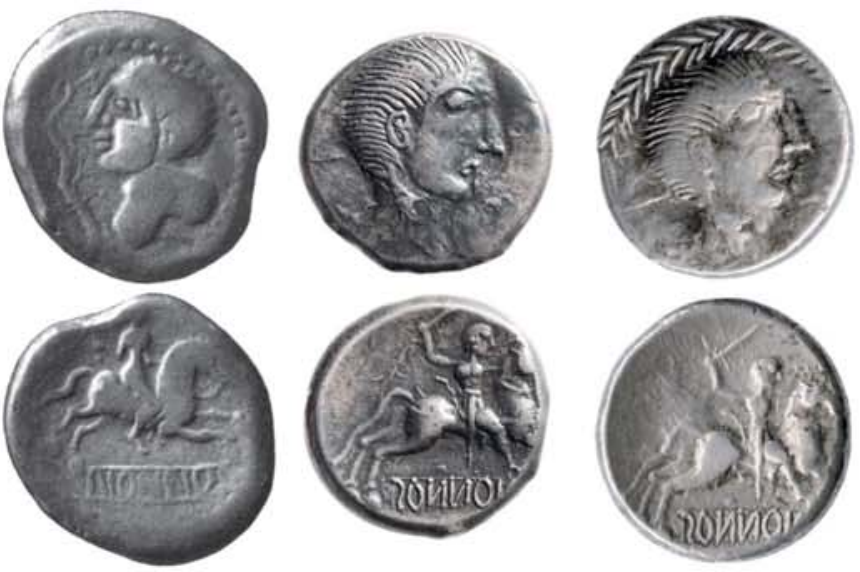

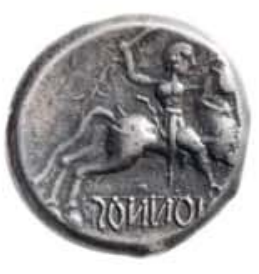

140

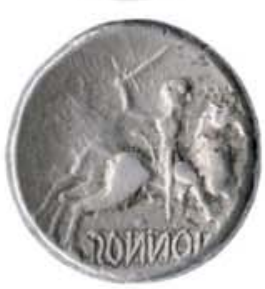

141

Fig. 4h. Deutsch Jahrndorf. Coins from the hoard (the numbers the correspond to catalogue; 1:1; photos by J. Militky and KHM Wien). - Obr. 4h. Deutsch Jahrndorf. Mince z pokladu (čísla odpovídaji katalogu; 1 : 1; foto J. Militký a KHM Wien).

AR tetradrachm, Biatec group - LATVMARVS (IANTVMARVS), ${ }^{18}$ var. Göbl (1994) XII/1-14

${ }^{18}$ Cf. chapter 5.2 for the new reading of the name as LATVMARVS.
Obv.: youthful bust with curly hair to right, on sides two large leaves, border of 'spruce' wreath

Rev.: clad rider on horse to right, holding rein, above tendril with leaves, around right and below LATVMARVZ 
Ref.: Paulsen 1933, Taf. 34: 792-794; Ondrouch 1958, nos. 240-243; Castelin 1978, no. 1162; Allen 1987, no. S242; Göbl 1994, Taf. 5: XII/1-14; Dembski 1998, Taf. 33: 633-635; Kostial 2003, no. 79; Ziegaus 2010, no. 488; Militký $2013 b$, no. 14

This coin: Röttger 2019, Database no. 29-13 / 1381

100. 17.02 g; $24.6 / 26.9 \mathrm{~mm} ; 3^{\text {h }}$ (MNM Budapest, inv. no. $14 / 1856.72$ )

-, This coin: Sacken 1862, 88, Fig. 17; Paulsen 1933, 158, no. 793, Taf. 34: 793; FRMÖ I/2, 329, no. 5/4/71, Taf. 2: 71; Dembski 1998, 82, no. 634, Taf. 33: 634; Röttger 2019, Database no. 29-21 / 483

101. $16.93 \mathrm{~g} ; 25.1 \mathrm{~mm} ; 8^{\mathrm{h}}$ (KHM Wien, inv. no. GR 27101)

-, This coin: Göbl 1994, Taf. 5: XII/1-14/2; Röttger 2019, Database no. 29-20 / 800.

102. $16.93 \mathrm{~g} ; 24.7 / 24.7 \mathrm{~mm} ; 4^{\mathrm{h}}$ (MNM Budapest, inv. no. $14 / 1856.73)$

-, This coin: Paulsen 1933, 158, no. 792, Taf. 34: 792; Pink 1974, Taf. VI: 83; FRMÖI/2, 329, no. 5/4/70, Taf. 2: 70; Dembski 1998, 82, no. 635, Taf. 33: 635; Röttger 2019, Database no. 29-26 / 482.

103. $16.89 \mathrm{~g} ; 26.0 \mathrm{~mm}$; $9^{\mathrm{h}}$ (KHM Wien, inv. no. GR 27100)

AR tetradrachm, Biatec group - NONNOS, var. Göbl (1994) XIV/1A-8

Obv.: diademed female head with elaborate hairdo to right, wavy line below neck, border of dots and arches turned inwards

Rev.: naked rider on horse to right holding sword in right hand, rein and branch in left hand, in exergue between two lines NONNOS, border of smooth line and arches turned inwards

Ref.: Forrer 1908, Taf. XXXVIII: 551; Paulsen 1933, Taf. 32: 764-766; Ondrouch 1958, nos. 136-137, 139, 141, 143-146, 148-149, 154-155, 161, 164, 176-178; Castelin 1978, no. 1159; Allen 1987, no. 245; Göbl 1994, Taf. 5: XIV/1A-8; Dembski 1998, Taf. 32: 617; Kostial 2003, no. 80; Militký 2013b, nos. 15-16

This coin: Forrer 1908, 348, no. 551, Taf. XXXVIII: 551; Paulsen 1933, 115, no. 1932; FRMÖ I/2, 328, no. 5/4/ 52; Röttger 2019, Database no. 32-1 / 915

Comment: origin of this coin from the hoard is not certain. The piece published by K. Castelin (1978, no. 1159) from the SLM collection in Zürich clearly differs from the drawing (Forrer 1908, Taf. XXXVIII: 551) and thus it does not come from the Deutsch Jahrndorf hoard

104. $17.47 \mathrm{~g}$ (Coll. R. Forrer, unknown present whereabouts)

-, This coin: Röttger 2019, Database no. 32-2 / 1233 105. 17.45 g; $24.8 / 28.0 \mathrm{~mm} ; 12^{\mathrm{h}}$ (MNM Budapest, inv. no. $14 / 1856.52)$

-, This coin: Röttger 2019, Database no. 32-4 / 1234 106. $17.38 \mathrm{~g} ; 25.6 / 26.4 \mathrm{~mm} ; 4^{\mathrm{h}}$ (MNM Budapest, inv. no. $14 / 1856.60)$

-, This coin: Röttger 2019, Database no. 32-8 / 1239
107. $17.34 \mathrm{~g} ; 25.0 / 25.5 \mathrm{~mm} ; 10^{\mathrm{h}}$ (MNM Budapest, inv. no. $14 / 1856.58)$

-, This coin: Röttger 2019, Database no. 32-9 / 1237 108. $17.33 \mathrm{~g} ; 27.1 / 25.6 \mathrm{~mm} ; 11^{\mathrm{h}}$ (MNM Budapest, inv. no. $14 / 1856.56)$

-, This coin: Paulsen 1933, 157, no. 766, Taf. 32: 766 (unprovenanced); Alföldi 1942, Taf. II: 5; Röttger 2019, Database no. 32-17 / 457

109. $17.28 \mathrm{~g} ; 24.6 / 24.9 \mathrm{~mm} ; 10^{\mathrm{h}}$ (MNM Budapest, Inv. no. $14 / 1856.37)$

-, This coin: Kress 91, no. 155; Button 95, no. 362; Button 107, no. 801; Münzzentrum Rheinland 145, no. 131; Röttger 2019, Database no. 32-23 / 35

Comment: information on the coin's origin comes from the auction catalogue (Button 107)

110. $17.21 \mathrm{~g} ; 26.7 / 24.1 \mathrm{~mm}$ (Coll. Ch. Röttger)

-, This coin: Röttger 2019, Database no. 32-28 / 1235 111. $17.19 \mathrm{~g} ; 24.9 / 25.1 \mathrm{~mm} ; 10^{\mathrm{h}}$ (MNM Budapest, inv. no. 14/1856.55)

-, This coin: Röttger 2019, Database no. 32-29 / 1236 112. $17.18 \mathrm{~g} ; 25.3 / 24.6 \mathrm{~mm} ; 10^{\mathrm{h}}$ (MNM Budapest, inv. no. 14/1856.59)

-, This coin: Röttger 2019, Database no. 32-36 / 1231 113. $17.17 \mathrm{~g} ; 26.3 / 24.9 \mathrm{~mm} ; 12^{\mathrm{h}}$ (MNM Budapest, inv. no. $14 / 1856.51)$

-, This coin: Röttger 2019, Database no. 32-44 / 1241 114. $17.14 \mathrm{~g} ; 24.1 / 25.6 \mathrm{~mm} ; 11^{\mathrm{h}}$ (MNM Budapest, inv. no. $14 / 1856.53)$

-, This coin: Röttger 2019, Database no. 32-43 / 1229 115. $17.14 \mathrm{~g} ; 24.5 / 23.9 \mathrm{~mm} ; 1^{\mathrm{h}}$ (MNM Budapest, inv. no. 14/1856.47)

-, This coin: Röttger 2019, Database no. 32-37 / 1240 116. $17.14 \mathrm{~g} ; 25.7 / 25.7 \mathrm{~mm} ; 12^{\mathrm{h}}$ (MNM Budapest, inv. no. 14/1856.39)

-, This coin: Paulsen 1933, 115, 157, no. 765 , Taf. 32: 765; FRMÖ I/2, 328, no. 5/4/50; Röttger 2019, Database no. $32-48$ / 456

117. $17.10 \mathrm{~g} ; 27.0 / 25.5 \mathrm{~mm} ; 12^{\mathrm{h}}$ (MNM Budapest Coll. V. v. Renner /Wien/, inv. no. 16/1955.13)

-, This coin: Röttger 2019, Database no. 32-27 / 1232 118. $17.08 \mathrm{~g} ; 24.8 / 28.6 \mathrm{~mm} ; 12^{\mathrm{h}}$ (MNM Budapest, inv. no. 14/1856.49)

-, This coin: Fiala 1900, 202, no. 2827; Paulsen 1933, 114, no. 1926; FRMÖ I/2, 328, no. 5/4/51; Röttger 2019, Database no. 32-61 / 1582

119. 17.02 g; $25.4 / 28.2 \mathrm{~mm} ; 12^{\mathrm{h}}$ (MNM Budapest Coll. Ernst Prinz zu Windisch-Grätz and K. Niklovits, inv. no. N I 4546)

-, This coin: Röttger 2019, Database no. 32-69 / 1320 
120. $16.95 \mathrm{~g} ; 25.1 / 26.6 \mathrm{~mm} ; 12^{\mathrm{h}}$ (MNM Budapest, inv. no. $14 / 1856.50)$

-, This coin: Röttger 2019, Database no. 32-78 / 1230 121. $16.85 \mathrm{~g} ; 24.9 / 29.0 \mathrm{~mm} ; 12^{\mathrm{h}}$ (MNM Budapest, inv. no. $14 / 1856.46)$

\section{AR tetradrachm, Biatec group - NONNOS,} var. Göbl (1994) XIV/1B-9

Obv.: diademed female head with elaborate hairdo to right, wavy line below neck, border of dots and arches turned inwards

Rev.: naked rider on horse to right, holding sword in right hand, rein and branch in left hand, in exergue between two lines NONNOS, border of smooth line and arches turned inwards

Ref.: Paulsen 1933, Taf. 32: 767-770; Ondrouch 1958, nos. 138, 140, 142, 147, 150-153, 156-160, 162-163, 165-175; Allen 1987, no. S238; Göbl 1994, Taf. 5: XIV/1B-9; Militký 2013b, no. 17

This coin: Röttger 2019, Database no. 33-3 / 1246 122. $17.48 \mathrm{~g} ; 26.7 / 23.8 \mathrm{~mm} ; 12^{\mathrm{h}}$ (MNM Budapest, inv. no. $14 / 1856.36)$

-, This coin: Röttger 2019, Database no. 33-6 / 1250 123. $17.38 \mathrm{~g} ; 29.1 / 24.3 \mathrm{~mm} ; 12^{\mathrm{h}}$ (MNM Budapest, inv. no. $14 / 1856.48$ )

-, This coin: Röttger 2019, Database no. 33-13 / 1245 124. $17.28 \mathrm{~g} ; 25.6 / 24.5 \mathrm{~mm} ; 12^{\mathrm{h}}$ (MNM Budapest, inv. no. $14 / 1856.38$ )

-, This coin: Röttger 2019, Database no. 33-15 / 1244 125. $17.27 \mathrm{~g} ; 27.1 / 24.9 \mathrm{~mm} ; 12^{\mathrm{h}}$ (MNM Budapest, inv. no. $14 / 1856.57)$

-, This coin: Paulsen 1933, 157, no. 768, Taf. 32: 768 (unprovenanced); Röttger 2019, Database no. 33-16 / 459

126. $17.26 \mathrm{~g} ; 24.4 / 26.8 \mathrm{~mm} ; 10^{\mathrm{h}}$ (MNM Budapest, inv. no. $14 / 1856.45)$

-, This coin: Röttger 2019, Database no. 33-14 / 1247 127. $17.27 \mathrm{~g} ; 25.8 / 24.5 \mathrm{~mm} ; 12^{\mathrm{h}}$ (MNM Budapest, inv. no. $14 / 1856.40$ )

-, This coin: Röttger 2019, Database no. 33-2 / 1249 128. $17.20 \mathrm{~g} ; 25.4 / 25.5 \mathrm{~mm} ; 12^{\mathrm{h}}$ (MNM Budapest, inv. no. $14 / 1856.54)$

-, This coin: Fiala 1900, 202, no. 2826, Taf. II: 2826; Egger 15, no. 50 (not depicted); Paulsen 1933, 115, no. 1927; Pink 1974, Taf. V: 75; FRMÖ I/2, 328, no. 5/4/53; Röttger 2019, Database no. 33-35 / 905

129. $17.12 \mathrm{~g} ; 24.7 / 24.6 \mathrm{~mm} ; 10^{\mathrm{h}}$ (MNM Budapest Coll. Ernst Prinz zu Windisch-Grätz and K. Niklovits, inv. no. N I 4547)

-, This coin: Röttger 2019, Database no. 33-36 / 1243 130. 17.09 g; $25.5 / 27.4 \mathrm{~mm}$; $9^{\mathrm{h}}$ (MNM Budapest, inv. no. $14 / 1856.42$ )

-, This coin: Röttger 2019, Database no. 33-34 / 1248
131. $17.08 \mathrm{~g} ; 25.4 / 24.4 \mathrm{~mm} ; 12^{\mathrm{h}}$ (MNM Budapest, inv. no. $14 / 1856.41)$

-, This coin: Paulsen 1933, 115, no. 1928 (?); FRMÖ I/2, 328, no. 5/4/54 (?); Röttger 2019, Database no. 33-58 / 1206

132. $16.93 \mathrm{~g} ; 25.8 / 25.3 \mathrm{~mm} ; 10^{\mathrm{h}}$ (MNM Budapest Coll. K. Niklovits, inv. no. N I 4548)

-, This coin: Röttger 2019, Database no. 33-78 / 1242 133. $16.47 \mathrm{~g} ; 24.5 / 24.7 \mathrm{~mm} ; 12^{\mathrm{h}}$ (MNM Budapest, inv. no. $14 / 1856.44)$

AR tetradrachm, Biatec group - NONNOS, var. Göbl (1994) XIV/1C-10

Obv.: diademed female bust with elaborate hairdo to right, border of dots and arches turned inwards

Rev.: naked rider on horse to right, holding sword in right hand, rein in left hand, in exergue between two lines NONNOS, border of smooth line and arches turned inwards

Ref.: Paulsen 1933, Taf. 33: 771-773; Ondrouch 1958, nos. 179-183; Allen 1987, no. 246, S239; Göbl 1994, Taf. 5: XIV/1C-10; Dembski 1998, Taf. 32: 619; Kostial 2003, no. 81

This coin: Röttger 2019, Database no. 35-18 / 1251

134. $17.24 \mathrm{~g} ; 26.0 / 25.3 \mathrm{~mm}$; $3^{\text {h }}$ (MNM Budapest, inv. no. $14 / 1856.35)$

-, This coin: Röttger 2019, Database no. 35-42 / 1608 135. $16.99 \mathrm{~g} ; 26.4 / 25.0 \mathrm{~mm} ; 3^{\mathrm{h}}$ (MNM Budapest - Coll. K. Niklovits, inv. no. N I 4550)

-, This coin: Fiala 1900, 202, no. 2825; Paulsen 1933, 114, no. 1929; FRMÖ I/2, 328, no. 5/4/55; Röttger 2019, Database no. 35-54 / 1610

136. $16.80 \mathrm{~g} ; 25.6 / 26.0 \mathrm{~mm}$; $9^{\mathrm{h}}$ (MNM Budapest - Coll. Ernst Prinz zu Windisch-Grätz and K. Niklovits, inv. no. N I 4549)

AR tetradrachm, Biatec group - NONNOS, var. Göbl (1994) XIV/3-11

Obv.: male bust with short hair to left, before on left branch terminating with pellets (mistletoe?), marked border of dots

Rev.: rider on horse to right, holding sword in right hand, rein in left hand, in exergue between two lines NONNOS, border of smooth line and arches turned inwards

Ref.: Paulsen 1933, Taf. 33: 774-777; Ondrouch 1958, nos. 184-193; Göbl 1994, Taf. 6: XIV/3-11; Dembski 1998, Taf. 31: 614-616; Kostial 2003, no. 85; Ziegaus 2010, no. 490; Militký 2013b, nos. 18-19

This coin: FRMÖ I/2, 328, no. 5/4/58, Taf. 2: 58; Dembski 1998, 81, no. 614, Taf. 31: 614; Röttger 2019, Database no. 38-2 / 767; Militký 2020, 314-315, Abb. 9.9: 10 137. $17.23 \mathrm{~g} ; 27.0 / 24.3 \mathrm{~mm}$; $1^{\mathrm{h}}$ (KHM Wien, inv. no. GR 27091)

-, This coin: Paulsen 1933, 157, no. 775 , Taf. 33: 775; FRMÖ I/2, 328, no. 5/4/57, Taf. 2: 57; Dembski 1998, 81, no. 615, Taf. 31: 615; Röttger 2019, Database no. $38-4$ / 465

138. $17.22 \mathrm{~g} ; 26.6 \mathrm{~mm} ; 10^{\mathrm{h}}$ (KHM Wien, inv. no. GR 27092) 
-, This coin: Chabouillet 1889, 243, no. 10153; De La Tour 1892, Pl. LIV: 10153; Paulsen 1933, 115, 157, no. 774, Taf. 33: 774; FRMÖ I/2, 328, no. 5/4/56; Röttger 2019, Database no. 38-18 / 464

139. $16.98 \mathrm{~g} ; 28.5 \mathrm{~mm}$ (Bibliothèque nationale de France, Paris, inv. no. 10153)

AR tetradrachm, Biatec group - NONNOS, var. Röttger (2019) XIV/2 (1)-13

Obv.: male head with short hair to right, border of 'spruce' wreath

Rev.: rider on horse to right, holding sword in right hand and oval shield in left hand (suggested by oval line around horse's head), in exergue २ОИИОИ

Ref.: Paulsen 1933, Taf. 32: 758-763; Ondrouch 1958, nos. 194-215, 265-267; Castelin 1978, no. 1158; Göbl 1994, Taf. 6: XIV/2 (1)-13; Dembski 1998, Taf. 31: 609613; Kostial 2003, nos. 82-84; Ziegaus 2010, no. 489; Militký 2013b, no. 20

This coin: FRMÖ I/2, 328, no. 5/4/47, Taf. 2: 47; Dembski 1998, 81, no. 610, Taf. 31: 610; Röttger 2019, Database no. 37-7 / 451; Militký 2020, 314-315, Abb. 9.9: 11

140. $17.25 \mathrm{~g} ; 25.7 \mathrm{~mm} ; 2^{\mathrm{h}}$ (KHM Wien, inv. no. GR 27094)

-, This coin: Fiala 1900, 202, no. 2828; Sotheby 1923, no. 138 , Pl. V: 138; Paulsen 1933, 114, no. 1925; FRMÖ I/2, 328, no. 5/4/48; Röttger 2019, Database no. 37$17 / 1023$

141. $17.18 \mathrm{~g} ; 26.4 / 26.7 \mathrm{~mm} ; 4^{\mathrm{h}}$ (MNM Budapest - Coll. Ernst Prinz zu Windisch-Grätz and K. Niklovits, inv. no. N I 4543)

-, This coin: Röttger 2019, Database no. 37-21 / 1272 142. 17.15 g; $25.1 / 25.3 \mathrm{~mm} ; 3^{\mathrm{h}}$ (MNM Budapest, inv. no. $14 / 1856.61)$

-, This coin: Röttger 2019, Database no. 37-38 / 1252 143. $17.12 \mathrm{~g} ; 25.7 / 25.1 \mathrm{~mm} ; 11^{\mathrm{h}}$ (MNM Budapest, inv. no. $14 / 1856.70$ )

-, This coin: Röttger 2019, Database no. 37-45 / 1273 144. $17.10 \mathrm{~g} ; 24.5 / 25.0 \mathrm{~mm} ; 1^{\mathrm{h}}$ (MNM Budapest, inv. no. $14 / 1856.62$ )

-, This coin: Paulsen 1933, 114, 157, no. 763, Taf. 32: 763; FRMÖ I/2, 328, no. 5/4/49; Röttger 2019, Database no. 37-41 / 454

145. 17.09 g; $27.5 / 27.2 \mathrm{~mm} ; 12^{\mathrm{h}}$ (MNM Budapest Coll. Áldor, inv. no. 95/1907.5)

-, This coin: Röttger 2019, Database no. 37-63 / 1275 146. $17.08 \mathrm{~g} ; 22.7 / 26.1 \mathrm{~mm} ; 7^{\text {h }}$ (MNM Budapest, inv. no. $14 / 1856.65)$

-, This coin: Röttger 2019, Database no. 37-62 / 1270 147. $17.08 \mathrm{~g} ; 25.9 / 26.0 \mathrm{~mm}$; $3^{\text {h }}$ (MNM Budapest, inv. no. $14 / 1856.63)$

-, This coin: Röttger 2019, Database no. 37-47 / 1274 148. $17.08 \mathrm{~g}$; 25.8/24.6 mm; $9^{\text {h }}$ (MNM Budapest, inv. no. $14 / 1856.64)$
-, This coin: Röttger 2019, Database no. 37-83 / 1271 149. 17.03 g; 25.3/24.3 mm; $9^{\text {h }}$ (MNM Budapest, inv. no. $14 / 1856.66)$

-, This coin: Röttger 2019, Database no. 37-82 / 1254 150. $17.02 \mathrm{~g} ; 22.7 / 26.1 \mathrm{~mm} ; 7^{\mathrm{h}}$ (MNM Budapest, inv. no. $14 / 1856.67)$

-, This coin: Pink 1932, Taf. I: 3; Paulsen 1933, 157, no. 758, Taf. 32: 758; FRMÖ I/2, 328, no. 5/4/46, Taf. 2: 46; Dembski 1998, 81, no. 609, Taf. 31: 609; Röttger 2019, Database no. 37-91 / 449

151. $17.00 \mathrm{~g} ; 25.6 \mathrm{~mm}$; $8^{\mathrm{h}}$ (KHM Wien, inv. no. GR 27093)

-, This coin: Röttger 2019, Database no. 37-128 / 1253 152. $16.81 \mathrm{~g} ; 25.1 / 25.2 \mathrm{~mm} ; 4^{\mathrm{h}}$ (MNM Budapest, inv. no. $14 / 1856.68)$

-, This coin: Röttger 2019, Database no. 37-132 / 1255 153. $16.79 \mathrm{~g} ; 25.5 / 25.6 \mathrm{~mm}$; $1^{\text {h }}$ (MNM Budapest, inv. no. $14 / 1856.69)$

AR tetradrachm, Biatec group - NONNOS, unknown type

Ref.: -

This coin: Egger 14, 3, no. 43; Paulsen 1933, 115, no. 1933; FRMÖ I/2, 329, no. 5/4/59; Röttger 2019, Database no. 58-1 / 1209

154. $17.12 \mathrm{~g}$ (private collection, unknown present whereabouts)

AR tetradrachm, Biatec group - TITTO, var. Göbl (1994) XV/1-25

Obv.: youthful male (?) bust to left, before on left branch with pellets (mistletoe?) on right wavy twisted lined, marked border of dots

Rev.: Harpy standing to right, looking left, above TITTO, marked border of dots

Ref.: Paulsen 1933, Taf. 34: 810, 35: 811-813; Ondrouch 1958, nos. 249-251; Göbl 1994, Taf. 6: XV/125; Dembski 1998, Taf. 34: 635; Kostial 2003, no. 86 This coin: Szilágyi 1895, XXXIX (with depiction); Paulsen 1933, 158, no. 813, Taf. 35: 813 (unprovenanced); Kerényi 1959, Taf. 3: 9; Röttger 2019, Database no. 40-5 / 503

155. $17.08 \mathrm{~g} ; 25.6 / 25.5 \mathrm{~mm} ; 1^{\mathrm{h}}$ (MNM Budapest, inv. no. $14 / 1856.75)$

-, This coin: Röttger 2019, Database no. 40-8 / 1269 156. $17.06 \mathrm{~g} ; 24.7 / 26.1 \mathrm{~mm}$; $9^{\mathrm{h}}$ (MNM Budapest, inv. no. $14 / 1856.76)$

AR tetradrachm, Biatec group - unknown legend This coin: unpublished

157. unknown metrology (MNM Budapest, inv. no. 14/1856.2, unknown present whereabouts)

-, This coin: unpublished

158. unknown metrology (MNM Budapest, inv. no. 14/1856.7, unknown present whereabouts)

-, This coin: unpublished

159. unknown metrology (MNM Budapest, inv. no. 14/1856.12, unknown present whereabouts) 


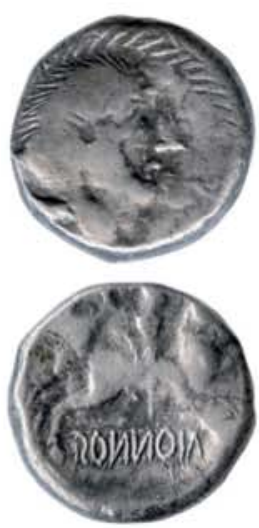

142
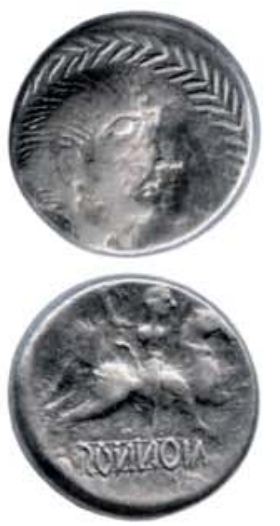

143
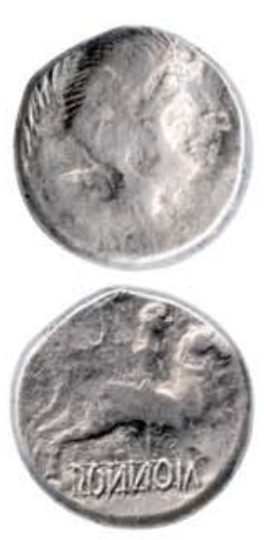

144
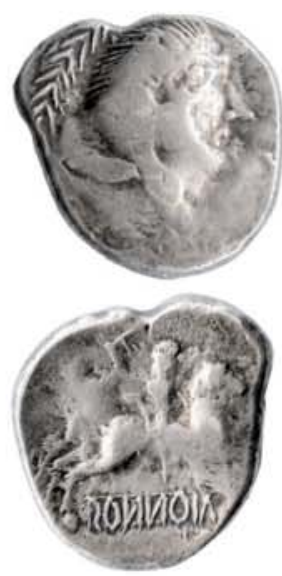

145
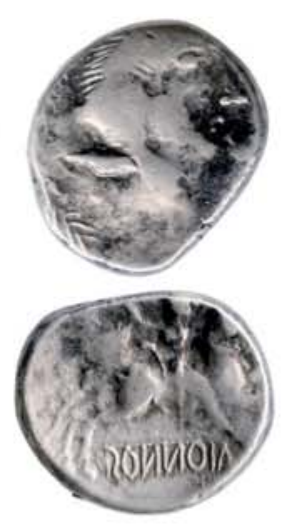

146
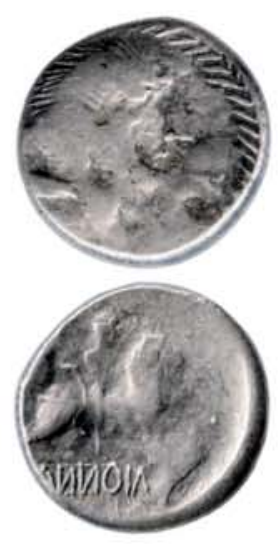

147

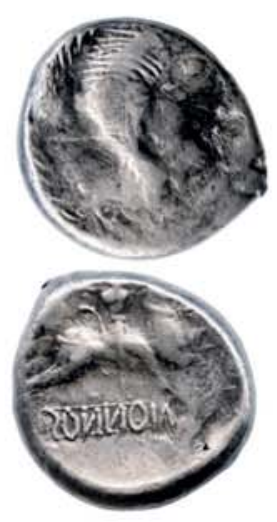

148
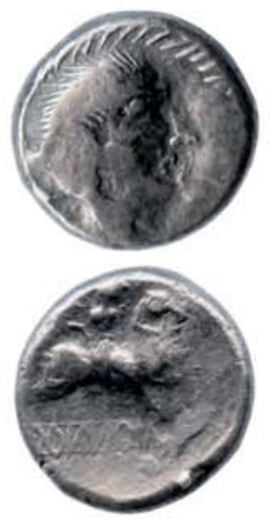

149
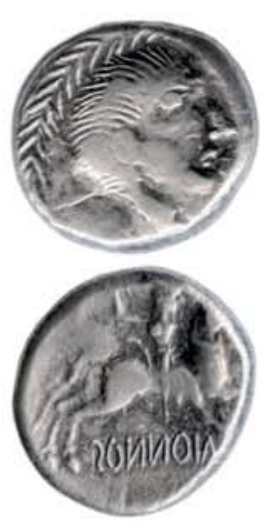

150
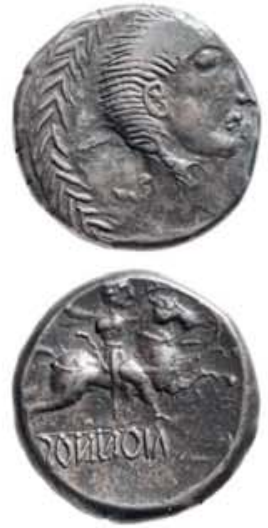

151
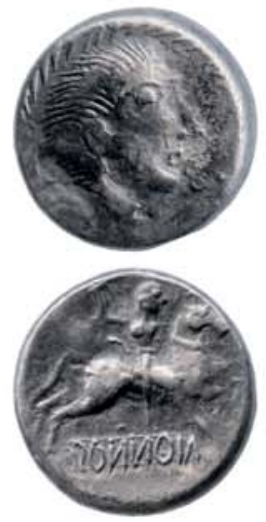

152
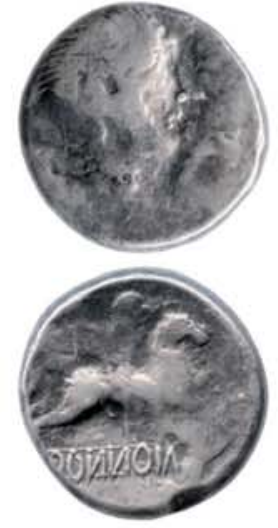

153

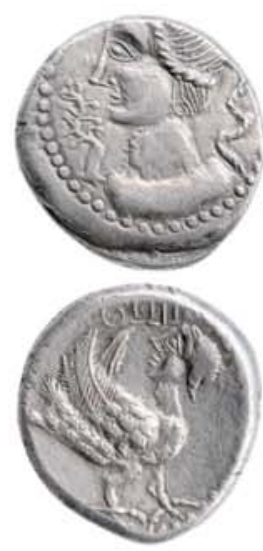

155

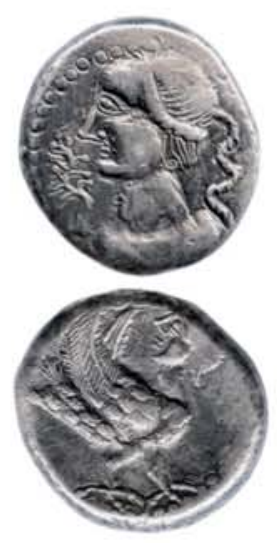

156

Fig. 4i. Deutsch Jahrndorf. Coins from the hoard (the numbers the correspond to catalogue; 1:1; photos by J. Militky and KHM Wien). - Obr. 4i. Deutsch Jahrndorf. Mince z pokladu (čisla odpovídaji katalogu; 1 : 1; foto J. Militký a KHM Wien).

-, This coin: unpublished

160. unknown metrology (MNM Budapest, inv. no. 14/1856.26, unknown present whereabouts)
-, This coin: unpublished

161. unknown metrology (MNM Budapest, inv. no. 14/1856.28, unknown present whereabouts) 
-, This coin: unpublished

162. unknown metrology (MNM Budapest, inv. no. 14/1856.43, unknown present whereabouts)

-, This coin: unpublished

163. unknown metrology (MNM Budapest, inv. no. 14/1856.77, unknown present whereabouts)

\section{The current state of knowledge on the Bratislava oppidum coinage}

\subsection{Basic characteristics}

The issue of the Bratislava coinage of the Biatec group, the Simmering type, and to a certain extent also the gold denominations has been given considerable attention, in particular in reaction to new discoveries (Kupido 1866; Kenner 1880; 1895; Eisner 1925). Synthesised studies of the topic have been presented by $R$. Paulsen (1933, 74-127, Taf. 28-43) and later V. Ondrouch (1958) in his study of the hoard discovered in Bratislava in 1942. The Bratislava gold issues have been treated by K. Castelin (1965, 15-16) who classified them to the phase D of his own relative-chronology system. Another synthesis was proposed by E. Kolníková (1991) mainly based on previously published material. The principal typology of the issues was penned by R. Göbl (1994). Although his typology is very detailed, his chronology is based only on fragile and not very realistic hypotheses (Göbl 1994, 13-25).

Over the last few years, the Bratislava coinage became the focus of intense research of several scholars. Among other things it was prompted by the new discoveries of Roman, or Romano-Celtic architecture in the Bratislava oppidum acropolis, the present-day Castle hill, which also yielded finds of coins (Musilová - Barta - Herucová /eds./ 2014; Musilová - Kolníková - Hložek 2015).

The state of knowledge was repeatedly synthesised by E. Kolníková (2012a; Kolníková - Bakos - Pauditš 2018, 157-159). The publication of the new hoard from the Romano-Celtic building I on the oppidum acropolis is of particular importance (Musilová - Kolníková Hložek 2015). Brief overviews were also presented by J. Militký within publications of two hoards from unknown sites (Militký 2013a; 2013b), within discussions concerning the finds of Simmering type coins from the Stradonice oppidum (Militký 2015a, 119-123), within a general synthesis of the Boii coinage (Militky 2015b, 89-91) and also in a monographic study of the La Tène period in Eastern Austria (Militký 2020, 313-318, Abb. 9.9). J. Fröhlich (2011) attempted an interpretation of the names present on silver issues in his publication of the stater with the counter-stamped (?) legend DEVIL. Ch. Röttger (2013) proposed a new reading of the inscriptions on some tetradrachms. The same author attempted to create a new relative and mainly absolute chronology (Röttger 2015). As will be demonstrated below, this scheme to a great extent contradicts the current state of knowledge of the Late La Tène chronology. Our idea of the distribution of the Bratislava coins is further complemented by several new finds published in Slovakia (Čambal - Budaj 2009; Budaj - C̆ambal $2013 a$; 2013b; 2016) as well as by a restudy of old finds (Ruske 201 1b; Vrtel - Maretta 2018; Röttger 2020).

The coins of the Biatec group are considered to be tetradrachms though they are termed hexadrachmas in R. Göbl's terminology (Göbl 1994), adopted also by other scholars (Kostial 2003, nos. 61-87; Militký 2004; 2013a; $2013 b ; 2015 a$ ). In reality however, their weight corresponds to that of tetradrachms in the traditional Attic standard (17.26 g) - the weight of the Bratislava coins mostly cluster slightly above $17 \mathrm{~g} .{ }^{19}$ Based on this, the weight of drachmas (not minted in Bratislava) would be $4.315 \mathrm{~g}$ and hemidrachms would weight $2.15 \mathrm{~g}$ which is exactly the value recorded for the Simmering type coins (Paulsen 1933, Taf. 36-42, 43: 971-978; Göbl 1994, Taf. 8: 23-33). ${ }^{20}$ These latter ones are named after the Vienna-Simmering hoard in which they were present (Kenner 1880; 1895; Paulsen 1933, 117-119; Ruske 2011a, 71, no. V27). During the production of the Biatec group tetradrachms, the traditional Attic standard was used for the Athenian tetradrachms of the so-called New style (Thompson 1961$)^{21}$ or for the tetradrachms issued on the island of Thasos (Thompson 1961) as well as for their barbarian imitations (Lukanc 1996), which as a rule weigh less than $17 \mathrm{~g}^{22}$ Two such Thasos imitation coins were documented in our area of interest in the Reca hoard (Paulsen 1933, 107-109, Taf. A: 17-18; Ondrouch 1964, 42, no. 57, 52, no. 91 and 68, no. 133). It is interesting to realise that the Bratislava moneyers opted to produce the very specific tetradrachms using the Attic weight standard rather than to assimilate to the clearly lighter 14 to 10 gram tetradrachms produced in Pannonia, in Noricum or by the Taurisci. In any case we are observing the creation of a specific silver denomination, reflecting the 'silver zone' of Celtic coinage (Milit$k y$ - Karwowski 2013, 29-30). We still do not grasp the reasons for the adoption of the Attic weight standard for the tetradrachm production; equally unclear is their monetary function in an environment in which they were surrounded by other Celtic denomination systems.

Our understanding of the monetary system is fully based on the finds of these coins. It is beyond any doubt that the centre of their production was the oppidum in present-day Bratislava (e.g. Pieta 2008, 111 ; Kolníková 2012a, 206-213). Apart from numerous individual coin finds, there are at least eight, and possibly nine coin hoards containing coins of the Biatec group (Ondrouch 1964, 44-46, nos. 61-66; Kolníková 1991, 23-27; Militký 2004; Kolníková 2012a, 215; Fig. 5). The oppidum,

\footnotetext{
19 It is worth stressing that both Slovak numismatists (V. Ondrouch, E. Kolníková) and e.g. B. Ziegaus (2010) systematically term these issues as tetradrachms.

20 The coins of the type Simmering are also sometimes labelled as drachmas (e.g. Kostial 2003, nos. 849-868; Ziegaus 2010, nos. 493-494; Militký 2015a, 119-123; Musilová-Kolníková-Hložek 2015, 256).

${ }^{21}$ Only exceptional finds are documented in Central Europe, e.g. in Jihlava (Nemeškalová-Jiroudková 1970).

22 Also tetradrachms issued in the name of Alexander III (336-323 $\mathrm{BC}$ ) use the Attic standard for a long time after his death (Price 1991). However, in the $1^{\text {st }}$ century BC these were not in circulation any more and therefore cannot be associated with the Bratislava coinage.
} 
with its inhabited area of approximately 98 ha (Vrtel 2011,265 , Fig. 1) has been studied mainly by means of numerous rescue excavations (for the site cf. Pieta-Zachar 1993; Čambal 2004; Pieta 2008, 111; Šedivý - Štefanovičová /eds./ 2012, 161-190). In 2009, masonry structures built in the Roman way were discovered in the Castle hill, i.e. the oppidum acropolis (Šedivý - Štefanovičová /eds./ 2012, 197-205; Musilová - Barta Herucová /eds./ 2014; Musilová - Kolníková - Hložek 2015).

It was in the interior of the structure I that the so far latest coin hoard was found. Besides silver coins of the group Biatec and type Simmering it included principally gold staters (Musilová - Kolníková - Hložek 2015, 253256, Fig. 13). The coins, originally considered to be a foundation offering, were discovered in a layer (no. 7) on top of the pavement, i.e. in the earliest stratigraphic unit filling in this part of the structure's interior (Musilová - Kolníková - Hložek 2015, 247-251, Fig. 7-10). The coins ended up in this place under unknown circumstances by the time of the buildings demise. Their distribution in the layer suggests a secondary position, e.g. set in the upper part of the building's wall. In any case the find proves that the buildings date to the period in which coins of the Simmering and Biatec group were being produced at the site. Coin production in the Bratislava oppidum is attested also by numerous coin finds in other parts of the oppidum as well as by finds of crucibles and flan moulds (Ščasnár - Kliment - Červeňanský - Zachar 1984; Kolníková 2012b, 34-39).

The monetary system of the Bratislava oppidum consisted of both silver and gold denominations; this was the case already in the monetary systems established around the middle of the $3^{\text {rd }}$ century BC in the Amber route corridor and in Bohemia (Militký 2015b, 71-77; 2019). Such bimetallic monetary systems were in vigour also during the oppida period in Bohemia and Moravia (Militký 2015a; 2015b, 79-87) and it is obvious that concurrent and mutually interdependent use of silver and gold was characteristic also of the Bratislava coinage as exemplarily demonstrated by coin hoards. Therefore, Eva Kolníková's idea that the production of gold staters, both epigraphic and anepigraphic, preceded the introduction of silver tetradrachms, should be turned down (Kolníková 2012a, 206; Musilová - Kolníková - Hložek 2015, 273-274). This hypothesis is clearly contradicted by the testimony of the hoards from Deutsch Jahrndorf and from the Bratislava oppidum acropolis (Musilová - Kolníková - Hložek 2015, 253-256, Fig. 13). It is absolutely unrealistic to accept Kolníková's idea of a Norican origin of the Karlstein type obols and their dating only to the latest phases of the oppidum (most recently cf. Kolníková - Bakos - Pauditš 2018, 159). The overall typology of small coinage of the oppida period in Bohemia, Moravia, the Transdanubian part of Austria, or in Trenčianské Bohuslavice (summarised e.g. in Militky 2015a,84-88) make it clear that the coins of the type Karlstein were certainly minted throughout the entire later phase of the oppida period (LT Dlb) ${ }^{23}$

\footnotetext{
23 The Slovak research sometimes labels this phase as D2 (e.g. Budaj - Čambal 2016).
}

and therefore throughout the entire lifetime of the Bratislava oppidum. They very simply formed part of the same bimetallic monetary system constituted partly by the traditional so-called 'Boii' denominations (gold coins and the Karlstein type) and partly by the specifically local denominations (Biatec group tetradrachms, 1/3-tetradrachms of the Biatec group, and the Simmering type hemidrachms). The presence of the Boii in Bratislava is an issue apart which cannot be discussed based on numismatic evidence itself.

\subsection{The denominations of the Bratislava oppidum monetary system}

Beginning in the pre-oppida period (LT C), the Celtic coinage in the so-called 'Boii area' was based on a bimetallic system of gold and silver denominations, appearing around the middle of the $3^{\text {rd }}$ century in the Amber route corridor and also in Bohemia (Militký 2019). In the oppida period Bohemia, three gold denominations (stater, 1/3-stater, and 1/8-stater) were minted together with silver obols (Militký 2015a, 43-44). Moreover, the traditional 1/24-staters kept being produced in Moravia, in particular at the oppidum of Staré Hradisko (Militký 2015b, 86-87).

The coin system of the Bratislava oppidum consists of several gold and silver denominations dated to the phase D of Karel Castelin's relative chronology (Castelin 1965, 15-16), which is the latest phase of Celtic coin production in Central Europe. The gold denominations, directly derived from the gold coinage produced in Bohemia, are represented by shell-type staters - either without a legend or with the legend BIATEC - and their sub-denominations produced on only a very limited scale; also, these 1/3-staters and 1/8-staters include both anepigraphic specimens and those bearing a legend abbreviated as BIAT. These issues will be analysed in detail including their new typology below in the study of the Deutsch Jahrndorf hoard. ${ }^{24}$ When discussing the gold epigraphic issues, it is also worth recalling the four staters inscribed NONNOS discovered in the hoard from the Bratislava oppidum acropolis (Musilová - Kolníková - Hložek 2015, 255, nos. 10-13, Fig. 13: 10-13; Fig. 7b: 19-22). Significantly, the gold denominations were found in the hoard together with tetradrachms and with the Simmering type hemidrachms. This is another proof confirming the concurrent use of all these denominations within a single monetary system.

It is hard to estimate the monetary relation between gold staters and silver teradrachms though it very probably reflected the Roman gold-silver ratio which settled during the reign of Augustus at 1:12.5. In this case, one stater would roughly correspond to five tetradrachms and 40 hemidrachms of the Simmering type.

The inscriptions BIATEC and NONNOS on the gold denominations, showing their connection with the silver denominations of the groups Biatec and Simmering have enormous interpretation potential. The hoards from Deutsch Jahrndorf (1855) and Bratislava (2009)

\footnotetext{
${ }^{24}$ Cf. chapter 5.1.
} 
as well as numerous finds of individual coins also bear testimony to an intense production of anepigraphic shell type staters in various types including their subdenominations. The role of these issues is exemplified first and foremost by the Deutsch Jahrndorf hoard and therefore much attention is dedicated to them in the following text. As will be shown, the anepigraphic staters are intimately linked with the latest phase of the Stradonice oppidum (Militký 2015a,49).

The most prominent coin type minted in Bratislava are without any doubt the silver tetradrachms of the Biatec group. These coins based on the Attic weight standard are absolutely unique within the Central European Celtic coin production in terms of their denomination, but also from the iconographic and epigraphic point of view. The most eye-catching characteristic of these coins are the personal names written in Latin script, as a rule on the reverse - AINORIX, BIATEC, BVSV, BVSSVMARVS, COBROVOMARVS, COISA, COVIOMARVS, COVNOS, DEVIL (or DEVII), EVOIVRIX (or FAPIARIX), FARIARIX (or FAPIARIX), ${ }^{25}$ LATVMARVS (IANTVMARVS), MACCIVS, NONNOS, TITTO (Göbl 1994, Taf. 1) and LATTV (Militký 2013b, 162, nos. 21-22, Fig. 1c: $21-22$; 2014). ${ }^{26}$ There is also one anepigraphic anonymous tetradrachm (Göbl 1994, var. XVI/1-17). The Latin legends bear a clear testimony to the influence of the Roman Republican milieu on the elites issuing coins in Bratislava. New light on this topic was shed by the discovery of Roman architectural features in the Bratislava oppidum acropolis. Within the Celtic coin production, a similar phenomenon can be observed in Noricum with its centre in the oppidum of Magdalensberg in which local coin production was based $(\mathrm{Krm}$ nicek 2010) and in which Roman-style masonry buildings were present in the Late La Tène period context. It was very probably at this site that numerous types of silver tetradrachms with Latin legends were minted: ADNAMATI, ATTA, BOIO, COPPO, COPO, COGESTLVS, CONGES.A, ECCAIO, ECCAIOS, EC/EICCAIO, ELVIOMAR, ESCINGOMA, NEMET, SVICCA, TINCO, and VOKK (Göbl 1973, 83-97, Taf. 1-18). Already before the Roman occupation of the Alps in $15 \mathrm{BC}$ when Noricum became Rome's client kingdom, the Magdalensberg was under a strong Roman influence. ${ }^{27}$ Although the chronology of the Norican coinage is a very complicated and by far not clear topic, it surely overlapped with the occupation of the Bratislava oppidum. The use of script on coins seems to have been one of the significant manifestations of Roman cultural infiltration into the Middle Danube Celtic area.

The iconography of the Biatec group tetradrachms is also entirely unique within the Celtic coinage. In a few cases, the models of these coins can be clearly identified

${ }^{25}$ An alternative reading proposed for the legend FARIARIX is FAPIARIX (Röttger 2013, 37).

${ }^{26}$ A detailed analysis of most variants of the Biatec group tetradrachms is provided in the discussion on the Deutsch Jahrndorf hoard.

27 At the same time, some Norican names suggest contacts with the Boii: Boio, Elviomar[us] or Escingoma[rus] (Kolníková 1991, 44). Also, the Boii military activities in Noricum in 58 BC suggest this may be the case (Dobiáš 1964, 29). in Roman Republican denarii minted between 80/71 and 46 BC (Göbl 1994, 13-21; Kolníková 2012a, 208; Militký 2013b, 167, tab. 1; Tab. 2). This provides them with the most precise post quem date of all Celtic issues in Central Europe. The beginning of their production can be placed sometime in the $60 \mathrm{~s} \mathrm{BC}$. It is impossible to account for the fact that the heavy silver coins in the Attic weight standard were minted with images adopted from Roman Republican denarii. The reasons could have been purely aesthetic or other. In any case, the knowledge of Roman Republican models is clear beyond any doubt. The imitation of Roman Republican coins is closely linked to the issue of their presence in Bratislava itself. Eva Kolníková (Musilová - Kolníková - Hložek 2015, 278, tab. 6) lists nine denarii and two bronze denominations found in the city area. Of key importance are two denarii discovered directly on the floor of the Roman building II: a denarius type $R R C$ no. $237 / 1$ a dated to $136 \mathrm{BC}$ and $R R C$ no. $387 / 1$ issued in $77 \mathrm{BC}$ (Resutík 2016, 113-114). The latter (and later) of these coins provides a useful post quem date for the use of the Roman building II which also yielded a lot of 16 hemidrachms of the Simmering type, discovered on its floor (Resutík 2016, 113).

Many of the motifs depicted on the Biatec group tetradrachms are specifically Celtic and have no parallels elsewhere (e.g. Kolníková 2012a, 208-209). They represent rare insights into the complex Celtic spiritual world which cannot be understood correctly. The most intensely produced coins seem to have been those under the names of BIATEC and NONNOS; the other issues are by far less numerous. It is not at all clear whether each specific name was minted only in a single time-span or if one name could reappear after a pause in which coins with other names were minted. The latter option seems more likely.

As already mentioned, the iconography of the Biatec group tetradrachms is so specific, that it is only thanks to the epigraphic specimens of gold staters and their sub-denominations that these coins can be linked with the Boii coinage. It is not by accident that some earlier collection catalogues listed the Biatec group tetradrachms and the Simmering group hemidrachms among the Eastern Celtic coinages (most recently: Allen 1987, 37-40, 65, 74-76, nos. 162-165, 241-251, S164-165, S235-242). In spite of the remarkable volume of their production, the Biatec group tetradrachms and Simmering type hemidrachms represent only a marginal phenomenon within the Boii coinage - as denominations they already reflect the 'silver zone' of the Celtic coinage (e.g. Militký - Karwowski 2013, 29-30).

The interpretation of the names on the coins is an extremely complex issue: we do not know if they are the names of actual rulers or rather of magistrates periodically elected from the aristocratic milieu. Some form of royalty is suggested by the suffix -rix (Ainorix, Evoivrix, and Fariarix /or Fapiarix/), while the suffix -marvs (as in Bvssvmarvs, Cobrovomarvs, Coviomarvs, and Latvmarvs /Iantvmarvs/) means 'big' (Kolníková 2012a, 207). Their 'royal' character is questioned by a new interpretation which presents them as the names of elected magistrates similarly to the situation in Gaul (Fröhlich 2011, 14-19). This concept was to some degree 
Tab. 2. Roman Republican denarii - models of the Biatec group coins and their post quem dates. - Tab. 2. Římské republikánské denáry jako predlohy mincí skupiny Biatec a jejich datování post quem.

\begin{tabular}{|l|l|l|l|}
\hline & \multicolumn{1}{|c|}{ Göbl 1994 } & \multicolumn{1}{c|}{ After } & \multicolumn{1}{c|}{ Pattern } \\
\hline COISA & $\mathrm{Vl} / 1$ & $80 / 71 \mathrm{BC}$ & obv.: $R R C$ 381/1a or 401/1 \\
\hline BIATEC & $\mathrm{II} / 1(1,2 / 1-2,3,4), \mathrm{II} / 2(1-2), \mathrm{II} / 3$ & $70 \mathrm{BC}$ & obv.: $R R C$ 403/1 \\
\hline COBROVOMARVS & $\mathrm{V} / 1$ & $70 / 46 \mathrm{BC}$ & obv.: $R R C$ 403/1 or 463/1a-b \\
\hline BVSSVMARVS & $\mathrm{III} / 1$ & $79 / 68 \mathrm{BC}$ & obv.: $R R C$ 383/1a or 407/1 \\
\hline BVSV & $\mathrm{IV} / 1$ & $79 / 68 \mathrm{BC}$ & obv.: $R R C$ 383/1a or 407/1 \\
\hline NONNOS & $\mathrm{XIV} / 1 \mathrm{~A}-\mathrm{C}$ & $66 \mathrm{BC}$ & obv.: $R R C$ 410/1 \\
\hline BIATEC & $\mathrm{II} / 6-29$ & $68 \mathrm{BC}$ & rev.: $R R C$ 407/1 or 407/2 \\
\hline BIATEC & $\mathrm{II} / 6-29$ & $46 \mathrm{BC}$ & obv.: $R R C 464 / 1$ \\
\hline
\end{tabular}

admitted also by E. Kolníková (2012a, 212). Such an idea can be accepted in the sense that the moneyers were elected aristocratic supreme magistrates. On the contrary, the idea that they are merely the names of elected moneyers, similarly to the situation in Republican Rome is rather unlikely.

The Bratislava coinage also includes other denominations. The particular denomination corresponding to three hemidrachms of the type Simmering ( $11 / 2$ drachm) with the legends BIATEC and NONNOS was probably produced only on a limited scale. Only a single specimen is known of each of these coins (Paulsen 1933, Taf. 35: 829-830; Göbl 1994, var. II/6-29 and XIV/2 /2/-30; Kolníková 1995). They are so far completely absent in hoards and their role within the coinage system is not fully understood. Importantly, the type with the BIATEC legend (Göbl 1994, var. II/6-29) imitates the Roman Republican denarii type $R R C$ no. $407 / 1$ or $407 / 2$ (rev.) and $R R C$ no. 464/1 (obv.) minted in 68 and in $46 \mathrm{BC}$, which makes it the latest post-quem dated Bratislava issue, minted not before $46 \mathrm{BC}$ (Tab. 2).

The hemidrachms of the type Simmering were produced on a very large scale. ${ }^{28}$ These coins struck with numerous die variants range in weight between 2.0 and $2.4 \mathrm{~g}$. The obverses feature variations of a laurel wreath or a Y-like feature flanked with circles. The reverses always bear the image of a prancing horse to the left with various combinations of bead appendices beneath the belly. The most important distinguishing features are however the symbols above the horse: a bird, an empty circle, a circle with a cross or with a dot, a beaded rosette, a dot with short fine rays, and a crescent (Paulsen 1933, Taf. C: 1-21). The typology of these coins has been only partially elaborated so far (Kolníková 1991, 82, Fig. 17-24; Göbl 1994, Taf. 8: 23-33). An entirely new typology - as detailed as a combination of dies was established by Ch. Röttger (2019) based on his database, it has however, not been published yet. Even though the iconography of the Simmering type hemidrachms differs entirely from that of the Biatec group tetradrachms, both coin types can be convincingly associated. Besides their frequent mutual presence in hoards, the reverse of one hemidrachma variant bears the legend NONN (Paulsen 1933, Taf. 36: 831-841; Göbl

\footnotetext{
28 The term is derived from the Wien-Simmering hoard (Kenner 1895; Paulsen 1933, 117-119; Ruske 2011a, 71, no. V27) discovered in 1880. The Simmering type hemidrachms were represented there by 262 pieces and accompanied by 29 tetradrachms of the Biatec group.
}

1994, Taf. 8: 28; Budaj - Čambal 2013b), without any doubt the abbreviation of the name NONNOS. In this variant, the prancing horse is turned to the right. A hint at a legend resembling the letters 'IP' can be found also in another rare hemidrachma variety (Paulsen 1933, Taf. 36: 842; Göbl 1994, Taf. 8: 29) though it is impossible to establish with which of the known tetradrachms its production was parallel. The synchronisation of the production of the Simmering type hemidrachms with the Biatec group tetradrachms is based on their concurrent presence in large hoards: e.g. Reca 1905 (Paulsen 1933, 107-109; Ondrouch 1964, 42, no. 57, 52, no. 91 and 68, no. 133) or Bratislava 1923 (Eisner 1925; Paulsen 1933, 104-105; Ondrouch 1964, 45, no. 63). ${ }^{29}$ Little can be said about their inner chronology though we might hypothetically suppose the variety with the Y-like obverse motif to be later. Crucial from the chronological point of view is their common presence in the 2009 hoard (Musilová - Kolníková - Hložek 2015, 256, nos. 20-22, Fig. 13: 20-22) as well as the presence of 16 so far unpublished items on the floor level of the Roman structure II in the Bratislava oppidum acropolis (Resutík 2016, 113). The Simmering type hemidrachms are also documented by numerous individual finds.

The last component of the Bratislava oppidum monetary system was the Karlstein type obols, the system's smallest denomination. The obverse of these coins is smooth without any image, while the reverse bears the motif of a horse, most often turned to the left. The Karlstein type obols are documented both as (numerous) individual finds (Kolníková - Kovár 2010, 70, Fig. 1: 5-10; Musilová - Kolníková - Hložek 2015, 276, Tab. 5) and from a hoard, specifically from what was originally a purse, discovered during the excavations in the Castle court (Vrtel 2011, 268, Fig. 6-7; Lesák 2016, 120). The Karlstein type coins were first identified in the Karlstein hill-top site near Salzburg, where a hoard of 54 of these issues and two Magdalensberg type obols was discovered in 1905 (Kellner 1990, 181-183, nos. 2002-2067, Taf. 51-52). Based on this find, the type was for a long time considered to be of west Norican origin (Göbl 1973, Taf. 44: N; Kellner 1990, 181). This opinion was adopted also by E. Kolníková in her publication of Norican coins found in the Bratislava oppidum (Kolníková 1996, 3438). Already at this time, a clear discrepancy in the documentation was apparent: the 'Norican' Karlstein type obols were documented throughout Bratislava as individual finds unlike the genuinely Norican issues, pres-

\footnotetext{
${ }^{29}$ Cf. chapter 4.4 .
} 
ent as a matter of fact, only in a single hoard (Kolníková 1996, 10-11, 15-16). The publication of the Karlstein type obols hoard from the oppidum of Trenčianské Bohuslavice made their Boian origin entirely clear (Kolníková 1998, 25-27). Still, E. Kolníková has kept supporting the hypothesis of their Norican origin and up until today she dates their circulation to the latest phases of the Bratislava oppidum, i.e. beginning in the 40s BC (Kolníková - Bakos - Pauditš 2018, 159). This idea contradicts all the evidence according to which the Bratislava coinage functioned as a coherent self-contained monetary system, as well as the fact that these coins can be found everywhere in the oppidum as individually lost pieces (Musilová - Kolníková - Hložek 2015, 276, Tab. 5; Kolníková - Bakos - Pauditš 2018, 183). ${ }^{30}$ It is extremely significant for chronological considerations, that the Karlstein type coins are systematically present in LT D sites in Bohemia, where they are labelled 'type C (Stradonice/Karlstein)'. From among the published assemblages, we can name Stradonice where 127 varieties were documented forming 19 distinct groups (Militký 2015a, 84-88, 109-114, 462-549, nos. 1426-2055, TAB. 28-33), Kolo near Týnec nad Labem (Militký - Beneš 2016, 245-247, 258, nos. 43-62) or Žehuň (Militký 2018, 200, 219-220, nos. 127-138). The finds from Bohemia nicely illustrate the clear transition of obols from the type B (Stradonice) to the type C (Stradonice/Karlstein). Their production in Bohemia can be dated roughly to the $80 \mathrm{~s} / 60 \mathrm{~s}-40 \mathrm{~s} / 20 \mathrm{~s} \mathrm{BC}\left(\mathrm{Mi}^{-}\right.$ litký 2015a, 84). The Karlstein type obols are attested also in Moravia, principally in the oppidum of Staré Hradisko (Militký 2015a, 84); they are represented also in the oppidum of Trančianské Bohuslavice in Slovakia (Kolníková 1998, 25-27) and in Oberleiserberg in Austria (Militký 2011, 1198). The archaeological and numismatic context of the Danubian sites which yielded finds of the Karlstein type obols suggest their dating to the time-span of LT D1b-D2, similar to the evidence from Bohemia. The large-scale presence of the Karlstein type obols in central sites overlaps clearly with the extent of the Boii coinage. In the case of the Bratislava oppidum, the Karlstein type obols - along with the gold denominations - represent a clearly 'Boian' component of the local monetary system. They form part of the latest phase of the Boii coinage (LT D1b-D2) dated to the first and mainly the second third of the $1^{\text {st }}$ century BC (e.g. Militký 2013a, 108; 2015a, 84).

\subsection{Chronology of the Bratislava oppidum coinage}

The chronology of the Bratislava coinage is a complex issue. In order to study it properly, we have to take into account the archaeological find-contexts, the post quem dates of some of the Biatec tetradrachms imitating Roman Republican models (Tab. 2), and also relativechronological relations between the individual issues.

\footnotetext{
30 The relatively small quantity of the individual finds is caused by the fact that the oppidum area is entirely covered by the present-day city and therefore it is not accessible to metal detector surveys.
}

Paradoxically, even though we are dealing with the most precisely dated Celtic coins in Central Europe, there is little agreement about their chronology.

The most complex view so far was presented by R. Göbl (1994) who as part of his typological analysis of the Biatec group tetradrachms also outlined a very precise and detailed chronology of the individual issues. Dividing them into 15 horizons, he attempted to date them - basically to the precision of a single year - to the time-span of 51-41 BC (Göbl 1994, 27-33, Taf. 14). Göbl associated the end of coin production in Bratislava with the decline of the oppidum after the defeat in the war with the Dacians in 41 BC (Göbl 1994, 37-45). However, Göbl's absolute dating and his distribution of the issues in the single phases cannot be accepted without reservations: it is a purely hypothetical typochronological construct; even the relatively chronological sequence of the individual types as proposed by Göbl is far from certain.

A new chronological concept of the Bratislava coinage was recently published by Ch. Röttger (2015). In his opinion, the coin production can be divided into six phases dated to the time-span of ca. 40-9 BC. The author associated the phases with specific coin variants (Röttger 2015, 203-207). The very late dating of this scheme is based mainly on the assumption that the tetradrachms with depictions of heads, generally considered reflections of Late Republican coinage, imitate in reality the issues of Augustus (27 BC-AD 14). Another argument for the late chronology is a motif present on the reverse of two of the tetradrachms with the legend BIATEC (Göbl 1994, var. II/3-12 a II/5-12) and at the same time on a British bronze coin with the legend VER (Röttger 2015, Fig. 7), dated between 25 BC and AD 10 (Cottam et al. 2010, 131, no. ABC 2676). The similarity is obvious but we may legitimately assume that the English issue imitated earlier Bratislava models. There are numerous reasons to decisively reject the idea that the majority of Bratislava coin production could date to the Augustan period (Röttger 2015, 204206).

Such a late chronology contradicts the archaeological dating of the site, usually placed to the second and third quarter of the $1^{\text {st }}$ century BC. This is also the dating of the imported Roman amphorae (Kysela - Olmer 2014, 184) and glass vessels (Musilová 2012, 369, 371) unearthed inside the Roman buildings on the oppidum acropolis. Both of these find categories suggest the construction of the buildings before the 40s BC. Dating prevalently to the Augustan period is also contradicted by the total absence of Augustan coins or by Eraviscan coins imitating Roman Republican denarii in the Bratislava oppidum (Musilová - Kolníková - Hložek 2015, 278, Tab. 6; for Eraviscan coinage cf. Torbágyi 1984; Haupt - Nick 1997; Kolníková 2005; Torbágyi - Vida 2020). This is in particular apparent when comparing the Bratislava oppidum with the nearby hill-fort of Devín. This site, in which there is no doubt about a Roman military presence in the Augustan period (Harmadyová 2012, 271-272), yielded a hoard of Eraviscan coins imitating Roman Republican denarii deposited in a pottery vessel (Plachá - Fiala 1998) as well as 19 Augustan issues (Musilová-Kolníková-Hložek 2015, 278, 
Tab. 6). Moreover, neither Eraviscan nor Augustan coins are attested in any oppidum north of the Danube. This implies that we are dealing here with two different chronological phases: the first one is represented by gold and silver issues produced in the Bratislava oppidum, the other is represented by Eraviscan imitations of Roman Republican denarii whose production and finds prevalently date to the Augustan period. ${ }^{31}$

The very existence of two numismatic horizons makes it clear that the Biatec group tetradrachms cannot imitate Augustan coins simply because they are earlier than them. This is further supported by archaeological sources. The only undisputable date of the Biatec group coins is the post quem date of the Roman Republican denarii they imitate (Tab. 2), the latest model being dated to $46 \mathrm{BC}$ (Göbl 1994, 14, var. II/6-29). ${ }^{32}$

The present state of knowledge suggests that the beginnings of the tetradrachm production as well as of the entire Bratislava monetary system date to the 60s BC. Its end was associated by Göbl (1994, 37-45) with the late 40s BC when Boii and Taurisci were allegedly wiped out by the Dacian king Burebistas (Dobesch 1994; 1995; Pieta 2008, 45, 50). Also, the coin production was supposed to have come to a halt as a result of this event. The same chronological scheme was also outlined by E. Kolníková (1991, 51-53; 2012a, 215). $J$. Fröhlich recently argued that at least the atypical stater inscribed DEVIL could have been minted only in the Early Augustan period (Fröhlich 2011, 19-20). However, the coin is totally atypical, differing from all the other shell staters, its chronology is entirely uncertain, and - principally - there is no way of telling if it was actually minted in Bratislava. As a result, it is not at all sure if the production of the Biatec group coins continued until the $30 \mathrm{~s} \mathrm{BC}$. There are increasingly more indications suggesting that the threshold of ca. $40 \mathrm{BC}$ need not have been the date of the site's demise and therefore it cannot be ruled out that the production (?) or at least the circulation of the Bratislava coins still date to the 30s BC. This idea can be upheld e.g. by the brooch of the type Almgren 238 (Almgren 1923) allegedly found in association with Bratislava coins in the Devínská Kobyla site near Bratislava (Budaj - Čambal 2016, 275-277, Fig. 4). Circulation of the Biatec group tetradrachms down to the $40 \mathrm{~s} / 30 \mathrm{~s}$ BC is attested by the Gerlitzn hoard (Paulsen 1933, 123-124; Ruske 2011a, 63 , no. V6) in which the Bratislava and Norican tetradrachms were accompanied by a Roman Republican quinarius of the type $R R C$ no. 489/3, minted in 43-42 $\mathrm{BC}$ in Gallia Transalpine or Cisalpine. Its origin in the circum-alpine region may suggest that it had not circulated for a long time before its deposition. Although the hoard contained only 19 Bratislava tetradrachms, a wide array of variants is represented (BIATEC: Göbl 1994,

\footnotetext{
31 The beginnings of the Eraviscan coin production in the broader area of present-day Budapest in Hungary (ancient Pannonia) can be dated to the period around the second half of the $1^{\text {st }}$ century BC. The main production period began somewhat later and may have continued as late as the Augustan period (Torbágyi 1997, 16; 2008, 206; Torbágyi - Vida 2020, 51-54, 56-57).

32 The model of the tetradrachm with the legend COBROVOMARVS (cat. nos. 87-88) was also minted in 70 or $46 \mathrm{BC}$ (Tab. 2).
}

var. II/ 1 (1)B-1B, II/ 1 (1)B-1C, II/ 1 (2/2)-3, II/3-12, II/4-3, II/4-7; DEVIL: Göbl 1994, var. IX/1-20; NONNOS: var. XIV/1A-8, XIV/1B-9, XIV/1C-10, XIV/2 (1)13). Their minting in $43-42 \mathrm{BC}$ is highly probable, providing thus another important anchoring point of the entire monetary system. In synthesis, the Bratislava coinage dates to the $60 \mathrm{~s}-40 \mathrm{~s}$, potentially to the $30 \mathrm{~s} \mathrm{BC}$.

An issue on its own is that of the so called Norican phase of the Bratislava oppidum, outlined by E. Kolníková (1996). In reality, there are extremely few actual Norican coins in Bratislava (Kolníková 2012a, 214-215; Militký 2016, 106, Tab. 4) - basically only specimens from a single disturbed hoard unearthed on the acropolis's southern slope. It contained one Norican tetradrachm with the legend ADNAMATI, 38 obols of the types Eis and Magdalensberg, and two hemidrachms of the type Simmering (Kolníková 1996, 10-11, Fig. 3-5). Noticeably, there are almost no individually lost west Norican coins in the oppidum (Kolníková 2012a, 214215). As a matter of fact, except for the hoard, only a single Norican tetradrachm of the type COPPO is documented (Kolníková 1996, 11-12, Fig. 2: 1). The hoard can be very likely linked with the phase of the Roman buildings in the acropolis - this would correspond well with the probable beginnings of the production of the Magdalensberg type obols in around 50 BC (Gorini 2009, 122, Fig. 3). The local Simmering type hemidrachms in the hoard make a significant link between the Norican issues in the hoard and local coin production. When discussing the so called Norican phase it is important to reject the idea of the Norican origin of the Karlstein type obols. As stated above, these issues are nothing but typically Boian small change forming an integral part of the local coinage - they are definitely not imports from Noricum dated to the period after the demise of the Bratislava coin production, i.e. to the 30s20s BC. E. Kolníková recently admitted the Bratislava origin of the Karlstein type coins but insisted on dating them to the period following the end of the Biatec tetradrachm group production in the 40s BC (Kolníková - Bakos - Pauditš 2018, 159). This idea cannot be accepted either since it contradicts the existence of the Bratislava monetary system based on several denominations (Militký 2016, 105-106).

\subsection{The testimony of hoards containing the Bratislava coins and their classification}

The hoards containing Bratislava coins can be classified into five groups based on the types of coins present in them. The first group includes both gold and silver denominations (Fig. 5: 1-3). This is the case of the hoards of Deutsch Jahrndorf (all gold denominations and tetradrachms) and of Bratislava 2009 (staters, tetradrachms, and the Simmering type hemidrachms: Musilová - Kolníková-Hložek 2015). Another such hoard has recently come to our knowledge: an old find from Potzneusiedl in Burgenland, Austria, containing four staters, 30 tetradrachms and probably three hemidrachms type of the Simmering. ${ }^{33}$

\footnotetext{
33 The publication of the hoard is in preparation by J. Militký and M. Karwowski.
} 
The second group is represented by hoards containing only the Biatec group tetradrachms (Fig. 5: 4-20). It includes several hoards from Bratislava (Paulsen 1933, 103-105; Ondrouch 1964, 44-46, nos. 61-62, 64-66), Bielovce (Paulsen 1933, 110; Ondrouch 1964, 53, no. 96), from the surroundings of Salzburg (Militky 2013b), and a hoard from an unknown site (Militký 2013a). Furthermore, there are many small hoards containing 2-5 tetradrachms: Bratislava (Paulsen 1933, 105; Ondrouch 1964, 47, nos. 69-70), Bratislava-Rača (Čambal-Budaj 2009, 200-202), Bratislava-Vajnory (Paulsen 1933, 107; Ondrouch 1964, 48, no. 76), Bratislava - surroundings (Paulsen 1933, 105; Ondrouch 1964, 48, no. 75), Malacky (Paulsen 1933, 107; Ondrouch 1964, 50, no. 85), Pezinok 1 (Paulsen 1933, 107; Ondrouch 1964, 51, no. 88), Pezinok 2 (Čambal - Budaj 2009, 197-200), Spiš region (Paulsen 1933, 111; Ondrouch 1964, 53, no. 98), and Trenčín (Paulsen 1933, 107; Ondrouch 1964, 53, no. 97). In some of these cases we cannot exclude that the few documented coins originated from larger hoards which were either not identified or got dispersed.

In the third group of hoards, the Biatec group tetradrachms were associated with the Simmering type hemidrachms (Fig. 5: 21-28). This category is represented by the already discussed hoards Reca (Paulsen 1933, 107-109; Ondrouch 1964, 42, no. 57, 52, no. 91 and 68, no. 133), ${ }^{34}$ Bratislava 1923 (Eisner 1925; Paulsen 1933, 104-105; Ondrouch 1964, 45, no. 63), Wien-Simmering (Kenner 1880; 1895; Paulsen 1933, 117-119; Ruske 2011a, 71, no. V27), Bratislava 1927 (Ondrouch 1964, 45, no. 63a), Schottwien (Paulsen 1933, 119-120; Ruske 2011a, 69, no. V23; 2011b), Stupava (Paulsen 1933, 106-107; Ondrouch 1964, 49, no. 82), Trnava (Paulsen 1933, 106-107; Ondrouch 1964, 49, no. 82a) and Schwechat (Ruske 2011a, 69, no. V24). Also, in the hoard from Bratislava 2009 the Biatec group tetradrachms were associated with the Simmering type hemidrachms as repeatedly mentioned above (Musilová - Kolníková - Hložek 2015, 254, Fig. 13: 20-22) and it is another valuable proof of the contemporary circulation of both denominations.

The fourth group is represented by finds of only Simmering type hemidrachms (Fig. 5: 29-31). These rather infrequent single-type hoards include e.g. the assemblages from Modra or Jur pri Bratislave, which consisted of 10 Simmering type hemidrachms and one Kapos type drachma (Kolníková 1990). The rather obscure find from Slovenský Grob (it is unclear to what extent we are dealing with an original assemblage) contained 22 hemidrachms as well as a 1/8-stater of type T and AE coins of the type Liptovská Mara (Budaj- C̆ambal 2013a, Fig. 2). We may include among the hoards also the 16 hemidrachms found on the floor of the Roman building II on the Bratislava oppidum acropolis (Resutík 2016, 113).

The fifth group of hoards is represented by assemblages of the Karlstein type obols (Fig. 5: 32). Only a single assemblage is documented so far - the find from the excavation in the Bratislava castle court (Vrtel 2011, 268, Fig. 6-7; Lesák 2016, 120). Originally the contents

\footnotetext{
${ }^{34}$ It also contained two tetradrachms of the Thasos type.
}

of a purse, it is currently a cluster of coins held together by corrosion which have not been separated yet. In any case it can be interpreted as personal ready cash rather than as the hoarding of valuables. The Karlstein type coins are not attested in other find assemblages, ${ }^{35}$ though they are very often present as individually lost pieces in various find contexts throughout Bratislava (Musilová - Kolníková - Hložek 2015, 276, Tab. 5). This small change was apparently not intended for hoarding, this is the same case as in Bohemia and Moravia of the oppida period (LT D1; cf. Militký 2015a, 75-88, 115117). The hoard from Trenčianské Bohuslavice is an exception in that the Karlstein type obols were deposited there together with gold denominations (Kolníková 1998, 16-30, Fig. 2-3).

A case apart are the hoards in which the Biatec group tetradrachms or the Simmering type hemidrachms were deposited together with other issues. Directly on the acropolis of the Bratislava oppidum, there is the abovecited coin hoard including the Norican tetradrachm inscribed ADNAMATI, obols of the types Eis (13 pcs) and Magdalensberg (25 pcs), as well as two hemidrachms of the Simmering type (Kolníková 1996, 10-11, 25-34; Militký 2016, 106, Tab. 4: 1-39). This important find illustrates contacts with Noricum though it cannot be taken as a proof of the late so-called Norican phase of the Bratislava oppidum (Kolníková 1996, 50-51). Chronologically, this find is very probably to be associated with the phase of Roman type structures on the acropolis (Militký 2016, 105-106).

Other mixed hoards were discovered outside Slovakia. In the Gerlitzen hoard, there were - apart from the Biatec group tetradrachms - also Norican tetradrachms and a Roman Republican quinar dated to 43/42 BC (Paulsen 1933, 123-124; Ruske 2011a, 63, no. V6). One Biatec group of tetradrachms was represented among Norican coins in the find from Moggio in northern Italy (Paulsen 1933, 125-126). Tetradrachms of the Biatec group, an anepigraphic 1/3-stater, anepigraphic 1/8-staters, and a 1/8-stater with the legend BIATEC were present in the hoard from Zuglio Carnico in northern Italy, discovered in 1762. This find also contained numerous Norican coins. Unfortunately, it is only documented by imprecise drawings (Moreno /ed./ 2003). Much is unclear about the alleged hoard from Wien-Simmeringer Heide, which contained a DEVIL tetradrachm, a west Norican COPO tetradrachm, three hemidrachms of the type Simmering, one Tótfalu type drachm, and one Pannonian drachm (Paulsen 1933, 119; Ruske 2011a, 73, no. F2). Four Biatec group tetradrachms are attested also in the Tămădău Mare hoard in Romania where they accompanied imitations of Thasos type tetradrachms (Kolníková 1991, 26). This find documents the penetration of the Bratislava coinage to the east. A detailed analysis of the Bratislava coin finds outside the immediate hinterland of Bratislava is one of the most important tasks of the future numismatic research.

${ }^{35}$ One obol of the type Karlstein was allegedly associated with the Bratislava 1923 find (Paulsen 1933, Taf. 26: 615); the coins however most likely did not belong to the assemblage. 


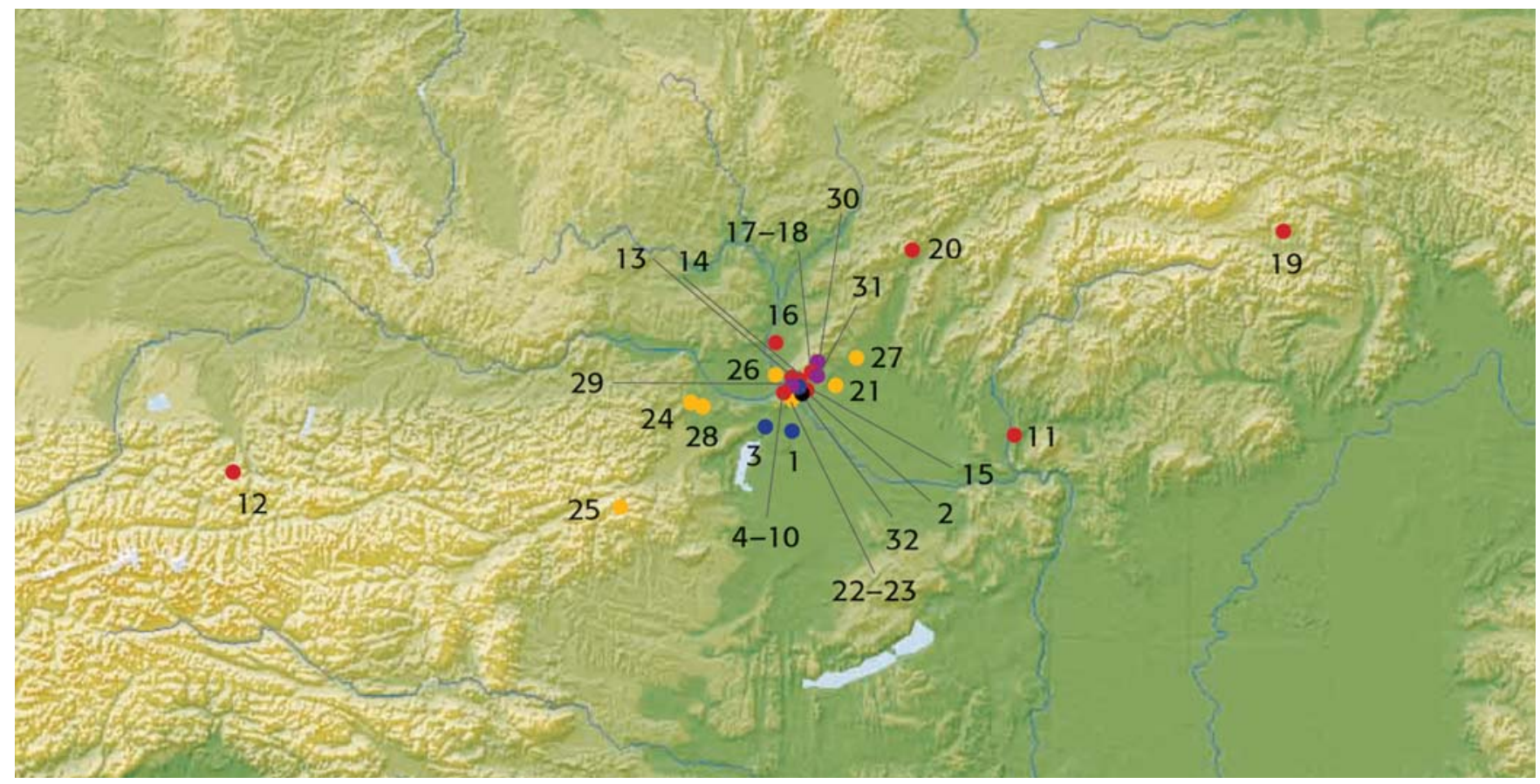

Fig. 5. Hoards and find assemblages containing Bratislava coins: blue circles - AV and AR denominations: 1 - Deutsch Jahrndorf; 2 - Bratislava $2009 ; \mathbf{3}$ Potzneusidl; red circles - AR tetradrachms of the Biatec group: 4-10 - Bratislava; 11 - Bielovce; 12 - Salzburg - surroundings; 13 - Bratislava-Rača; 14 Bratislava-Vajnory; 15 - Bratislava - surroundings; 16 - Malacky; 17-18-Pezinok; 19 - Spiš region; 20 - Trenčín; yellow circles - AR tetradrachms of the Biatec group and AR hemidrachms of the type Simmering: 21 - Reca; 22-23 - Bratislava; 24 - Wien-Simmering; 25 - Schottwien; 26 - Stupava; 27 - Trnava; $\mathbf{2 8}$ - Schwechat; violet circles - AR hemidrachms of the type Simmering: $\mathbf{2 9}$ - Bratislava; $\mathbf{3 0}$ - Modra/Jur pri Bratislave; $\mathbf{3 1}$ - Slovenský Grob; black circle AR obols of the type Karlstein: $\mathbf{3 2}$ - Bratislava. - Obr. 5. Depoty a hromadné nálezy obsahující bratislavské mince: modrá kolečka - AV a AR nominály: 1 Deutsch Jahrndorf; 2 - Bratislava 2009; 3 - Potzneusidl; červená kolečka - AR tetradrachmy skupiny Biatec: 4-10 - Bratislava; 11 - Bielovce; 12 - Salzburg - okoli; 13 - Bratislava-Rača; 14 - Bratislava-Vajnory; 15 - Bratislava - okoli; 16 - Malacky; $17-18$ - Pezinok; 19 - Spiš; 20 - Trenčín; žlutá kolečka - AR tetradrachmy skupiny Biatec a AR hemidrachmy typu Simmering: 21 - Reca; 22-23 - Bratislava; 24 - Wien-Simmering; 25 - Schottwien; 26 - Stupava; 27 Trnava; 28 - Schwechat; fialová kolečka - AR hemidrachmy typu Simmering: 29 - Bratislava; 30 - Modra/Jur pri Bratislave; $\mathbf{3 1}$ - Slovenský Grob; černé kolečko - AR oboly typu Karlstein: $\mathbf{3 2}$ - Bratislava.

\subsection{The raw materials of the Bratislava coinage}

A crucial question connected with the issue of the Celtic coinage in Bratislava is the provenance of the metal used for the coin production. The provenance of gold used for the minting of the Celtic coins in Bohemia was recently discussed to a large extent during the study of finds from the Stradonice oppidum (e.g. Militký 2015a, 156-158). A voluminous study has recently been published on this topic in Slovakia (Kolníková - Bakos Pauditš 2018, 144-145). There is so far no clear evidence for underground mining apart from some hints at sub-surface extraction in the Závist oppidum (Militký $2015 a$, 158). It is nevertheless beyond any doubt that gold was washed and subsequently distributed over long distances. Such practices are exemplified by natural gold nuggets from the Stradonice oppidum (Militky $2015 a, 158$, nos. T V/1-2). Both specimens could have reached the site either through trade with precious metals or as part of the raw metal supply arriving from the gold-washing regions, which might have been directly controlled by the Stradonice oppidum.

Gold was clearly a common trade commodity as early as in the $3^{\text {rd }}$ century BC, both raw and minted. This is clearly attested by the massive coin production going on already in the pre-oppida period in Moravia and Lower Austria (Militký 2019, 284-298), both being regions with only extremely limited local gold sources. The complexity of the issue of the origin of gold intended for coin production is well illustrated by the finds of gold ingots in Moravia (Fröhlich 2012, 157-158) and by hatched gold in various forms (coins among others) and of varying fineness from the trade and production site of Němčice nad Hanou (Fröhlich 2012, 150-155). The fact that unminted gold was the object of trade is also attested by finds from Lower Austria where hatched coins (Dembski 1999, 108-109, nos. 1-4) and an ingot (Dembski 2009, 92, Fig. B-11) were discovered along with hatched ingots and gold fragments (Dembski 1999, 109-110, nos. 7, 9-11). One of the ingots discovered in Roseldorf is a bar, hatched at the ends and with proof cuts - though in reality the whole is only a gold-plated copper fake (Dembski 1999, 109, no. 8; 2009, 93, Fig. B-13). It is a precious testimony of the fact that even hatched metal was occasionally faked; the only explanation is that even unminted metal was an object of trade.

The fact that raw gold was also traded in the oppida period is well documented by the $217 \mathrm{~g}$ gold lump from the Manching hoard (Ziegaus 2013, 559, Taf. 21: 484). The treasure contained 483 Boii staters in a wide array of types. This is the first case when raw gold was documented together with minted coins in Central Europe. It could mean that large payments in particular could have been carried out also in unminted metal, though at the same time the ingot as well as the coins could 
have been simply raw material meant for the further production of local gold coins in the Manching oppidum.

The origin of the gold used for the production of the Bratislava gold staters and their subdenominations is unknown. It could have come from all the above listed sources including coins, mainly those from Bohemia and Moravia. It is clear, nevertheless, that the gold must have been imported to Bratislava, most likely in the form of ingots. A gold ingot bar from Slovenský Grob is worth recalling here (Budaj-Čambal 2013a, 213, no. 25, Fig. 2: 25). Its weight of 6.57 g corresponds precisely with that of the shell staters minted in Bratislava though the fineness of the ingot is clearly higher: $99.1 \%$ while in the Bratislava staters from the 2009 hoard the fineness ranged from 92 to 84\% (Musilová - Kolníková Hložek 2015, 283, Tab. 7: 1-15). ${ }^{36}$ It is not sure that the ingot dates to the period in which coins were being produced in Bratislava though coins found with it - in particular the Simmering type hemidrachms - hint at that being the case (Budaj - C̆ambal 2013a, Fig. 2: 2-23).

Even more complex is the issue of the origin of silver in La Tène period Central Europe. Also, it was recently discussed for the case of the Stradonice oppidum (e.g. Militky 2015a, 156-157) and in the large scope study published for the case of Slovakia (Kolníková - Bakos Pauditš 2018, 145-146). Unlike gold, silver can be obtained only by underground mining. There is still no unequivocal evidence of silver mining in the La Tène period Czech lands (Militký 2015a, 157). Also, in the neighbouring regions direct evidence of silver mining is not fully convincing. Only indirect hints come from the mountainous regions of northern Slovakia (Pieta 2008, 74); moreover, the majority of deposited silver is contained in polymetallic ores. There is still no evidence for the complex process of silver refining in these regions. In Moravia and Lower Austria, silver mining in the La Tène period is not attested. And yet, silver coins were produced on a large scale in the Boii coinage regions already in the pre-oppida period and therefore silver must have been imported either in an unminted or in a minted form. Its accessibility in Bratislava is attested by the plentiful presence of silver coins of the group Biatec and type Simmering.

During the publication of the finds from the oppidum of Stradonice, various types of silver fragments have been documented and classified; they represent coin flans and their semi-products, as well as various casting spills present in large numbers (Militký 2015a, 154-158, 664-679). These finds bear a unique testimony to intense silver working in the oppidum, prevalently for monetary purposes. The collection of unminted silver from the oppidum of Trísov is very similar though incomparably less numerous. ${ }^{37}$

Silver bar ingots represent a category of its own. It appears that mainly in the oppida period (LT D) this category documenting intense trade with raw silver in a standardised form was extremely important. Two silver ingots are attested in Stradonice (Militký 2015a,

\footnotetext{
${ }^{36}$ Unfortunately it was not possible to analyse the coins of the Deutsch Jahrndorf hoard for the needs of the present study. 37 Their publication is in preparation by J. Militký.
}

156-157, 672-673, nos. T III/1-2), three in the oppidum of Třísov (Militký 2013a, 115-116, Fig. 3: 3-5), one in the hill-fort of Oberleiserberg in Lower Austria (Militký 2013a, 115, Fig. 3: 2). In several other cases, dating to the La Tène period is not certain and the ingots could be more recent (Militký 2013a, 116). It is clear nevertheless that ingots were widely recognised as the standard form of raw silver. The rarity of the documented cases clearly results from the fact that the absolute majority of them were melted down during coin production.

One highly interesting ingot is closely linked to the issue of the Bratislava coinage (Militky 2013a, 101, 103, no. 15, Fig. 2: 15); allegedly it formed part of a hoard of Biatec group tetradrachms from an unknown site $(\mathrm{Mi}-$ litký 2013a, 100-103, nos. 1-14, Fig. 1-4). It is a silver bar ingot of an oblong shape and a trapezoidal section (length $161 \mathrm{~mm}$, width $23.5 \mathrm{~mm}$, height $12.3 \mathrm{~mm}$ ), made by casting into an open mould. Its weight of 418.184 g does not correspond with any ancient weight unit (Fig. 6). ${ }^{38}$ The non-destructive XRF analysis of the alloy composition which was carried out in Munich discovered a fineness of 93-94\% with admixtures of copper and tin. Paradoxically, the composition of the ingot cannot be compared with that of the Bratislava coins since only a negligible amount of them have been analysed so far. Composition analyses have been carried out on four tetradrachms of the Biatec group and three hemidrachms of the Simmering type from the Bratislava 2009 hoard (Musilová - Kolníková - Hložek 2015, 283, tab. 7: 16-22). ${ }^{39}$ Even in this small sample, the obtained results were highly disparate: in the Biatec group tetradrachms the silver content ranged from 95.8 to $75.6 \%$ while in the Simmering type hemidrachms it was in the range of 96.8-86.4\%. The Simmering type hemidrachma from the Chaura collection kept in the Prague National Museum has a silver content of only 75\% and a much more varied array of admixtures (Militký - Vacinová 2012, 29, no. 30). It seems therefore, that the Bratislava silver coins are unusually varied in their composition. This trend however needs to be confirmed on the grounds of a statistically more robust sample.

It is sure that the oppidum of Bratislava did not control any local sources of precious metal - none can be found in its broad surroundings. It is almost sure that the metal was obtained first and foremost by trade. We can only speculate where the imported silver came from: the possibilities include sources in northern Slovakia, the Transylvanian deposits, and also the area of the Roman Empire (Militký 2013a, 117). This option appears particularly attractive. The contacts between Celtic Bratislava and the Roman Republic are exemplarily documented by the recent discoveries of masonry structures built by Roman craftsmen or at least under their supervision. The Roman imports to the site included numerous wine amphorae (Kysela-Olmer 2014), luxurious glass vessels (Musilová 2012), or Roman Re-

\footnotetext{
38 It could be very approximately assimilated to the weight of 1 and a $1 / 4$ of the Roman pound but even so it would be $10 \mathrm{~g}$ heavier. 39 Unfortunately it was not possible to analyse the coins of the Deutsch Jahrndorf hoard for the needs of the present study.
} 

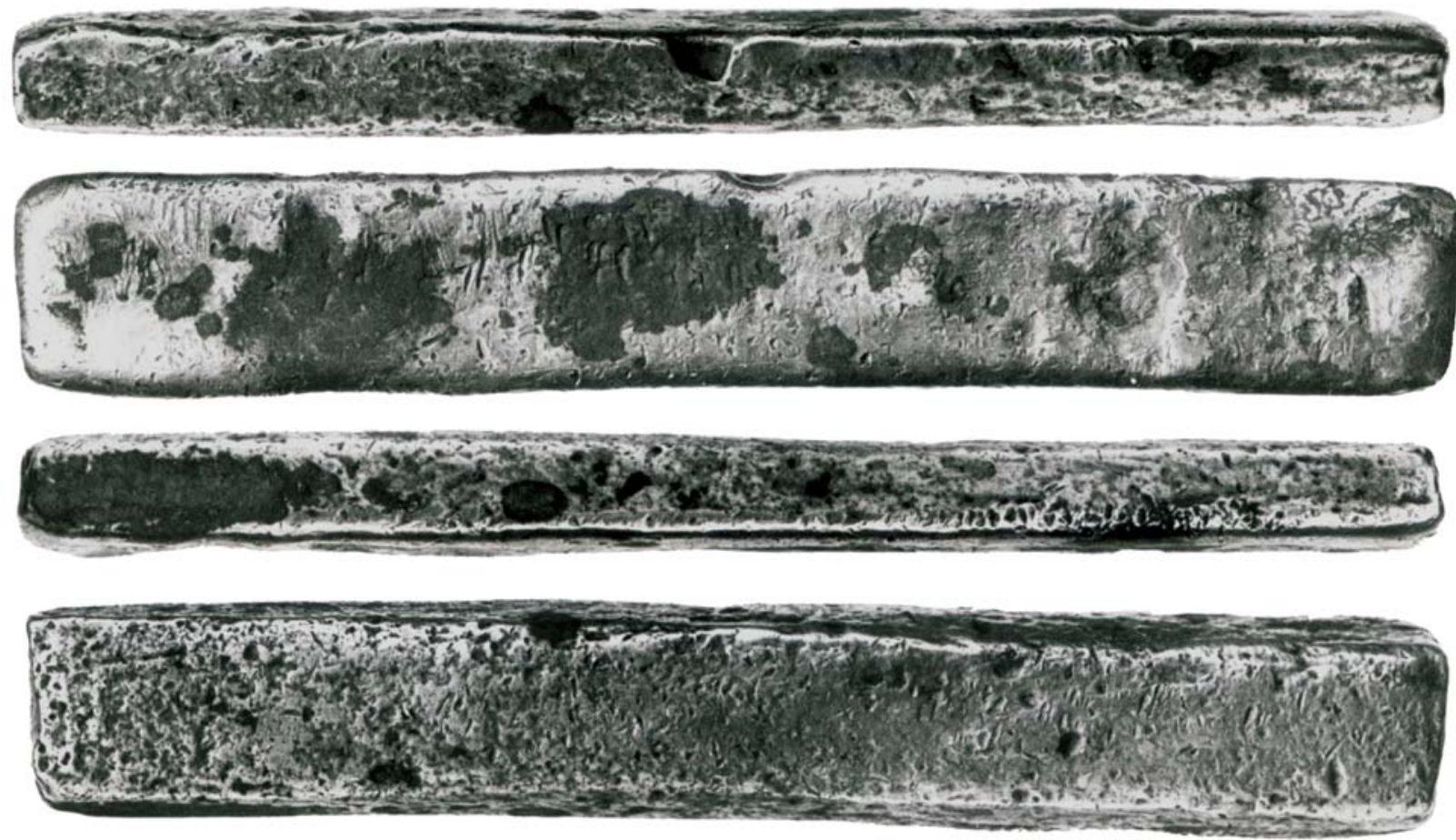

Fig. 6. A silver ingot from a hoard from an unknown site (after Militký 2013a, 103, Fig. 2). - Obr. 6. Stř́brný ingot pocházející z depotu z neznámé lokality (podle Militký 2013a, 103, obr. 2).

publican silver denarii (Kolníková - Bakos - Pauditš 2018,193 ) which could by themselves become raw material for local coin production. However, also unminted silver in the form of ingots could have been an object of trade. Local trade with raw metal in the form of ingots is well documented by a unique find of a $102 \mathrm{~cm}$ long and $11.9 \mathrm{~kg}$ heavy brass bar (Resutík 2016, 110-111). It is not sure whether this object was imported or whether it was on the contrary intended for export, though the option of a Roman import is more likely ( $R e^{-}$ sutík 2016, 111). Should this be the case, the idea of importing Roman silver in bars to the oppidum of Bratislava seems very probable.

\section{Numismatic analysis of the Deutsch Jahrndorf hoard}

\subsection{Gold coins}

The testimony provided by the gold coins from the Deutsch Jahrndorf hoard is absolutely unique. In order to fully understand their significance, we have to follow the larger context of the production of gold denominations during the oppida period (LT D) in the Boii coinage area, i.e. principally in the territory of Bohemia and Moravia. The Bratislava coins clearly follow the tradition of these gold Boii issues.

The typological classification of the Boii staters of the oppida period was established by K. Castelin (1965, 13-16) on the grounds of the earlier monograph by R. Paulsen (1933). Castelin defined the basic groups representing the relative chronological phases of the issues B-D. Our detailed knowledge of the gold denomi- nations of the phase B is largely based on four published hoards Großbissendorf (Ziegaus 1995), Manching (Ziegaus 2013), Starý Kolín (Nemeškalová-Jiroudková 1998), and Campiglia Marittima - San Vincenzo (Nemeškalová-Jiroudková 1975). Unfortunately, no large hoard is available for the phase $\mathrm{C}$, since the treasures from Podmokly (Militký 2015a, 34-35) and Stradonice 1877 (Militký 2015a, 31-32, 174-179, nos. C1-17) were mostly melted down. A complex view of the production of gold coins in Bohemia was provided by the publication of coins from the oppidum of Stradonice (Militký 2015a, 44-69, 186-251, nos. 18-253). In Slovakia, the late phase $\mathrm{C}$ is represented by the gold component of the hoard from the oppidum of Trenčianské Bohuslavice (Kolníková 1998, 12-18, Fig. 1-2). It is on the grounds of all these studies that we can understand and date the gold component of the Deutsch Jahrndorf hoard minted in the Bratislava oppidum.

The gold coins from the Bratislava oppidum consisted of several denominations which can be classified to the phase D of the K. Castelin (1965, 15-16) relative chronology; it is the latest phase of the production of gold Boii coins. Two groups can be distinguished between the gold denominations: anepigraphic coins and coins bearing the legends BIATEC and NONNOS. Gold issues of the Bratislava oppidum are still insufficiently studied. The largest quantity of gold coins (29 pcs; Tab. 3) come from the Deutsch Jahrndorf hoard; these are studied in detail in the following text. Another 15 staters were found in the Bratislava 2009 hoard discovered inside the Roman building I (Musilová - Kolníková Hložek 2015, 253-255, Fig. 13: 1-15). Four anepigraphic staters have very recently been documented in the Potzneusiedl hoard from Burgenland where they ac- 


\begin{tabular}{|l|l|l|l|c|}
\hline Cat. no. & \multicolumn{1}{|c|}{ Denomintion } & \multicolumn{1}{|c|}{ Var. } & Pcs \\
\hline 1 & stater & Var. I.1 & $6.43 \mathrm{~g}$ & $\mathbf{1}$ \\
\hline 2 & stater & Var. I.2 & $6.45 \mathrm{~g}$ & $\mathbf{1}$ \\
\hline 3 & stater & Var. I.3 & $6.18 \mathrm{~g}$ & $\mathbf{1}$ \\
\hline 4 & stater & Var. II.1 & $6.74 \mathrm{~g}$ & $\mathbf{1}$ \\
\hline $5-6$ & stater & Var. II.2 & $6.48-6.47 \mathrm{~g}$ & $\mathbf{2}$ \\
\hline $7-9$ & stater & Var. II.3 & $6.49-6.42 \mathrm{~g}$ & $\mathbf{3}$ \\
\hline 10 & stater & Var. II.4 & $6.44 \mathrm{~g}$ & $\mathbf{1}$ \\
\hline 11 & stater & Var. III.1 & $6.63 \mathrm{~g}$ & $\mathbf{1}$ \\
\hline 12 & stater & Var. IV.1 & $6.51 \mathrm{~g}$ & $\mathbf{1}$ \\
\hline $13-14$ & stater & Var. IV.2 & $6.49 \mathrm{~g}$ & $\mathbf{2}$ \\
\hline 15 & stater & Var. IV.3 & $6.46 \mathrm{~g}$ & $\mathbf{1}$ \\
\hline 16 & stater & Var. V.1 & $6.51 \mathrm{~g}$ & $\mathbf{1}$ \\
\hline 17 & stater & Var. VI.1 & $6.47 \mathrm{~g}$ & $\mathbf{1}$ \\
\hline 18 & stater & Var. VII.1 & $6.46 \mathrm{~g}$ & $\mathbf{1}$ \\
\hline 19 & $1 / 3-$ stater & Var. 1/3-E/VI:02 & $2.25 \mathrm{~g}$ & $\mathbf{1}$ \\
\hline $20-21$ & $1 / 3-$ stater & Var. 1/3-F/VII:01 & $2.14-2.10 \mathrm{~g}$ & $\mathbf{2}$ \\
\hline 22 & $1 / 3-$ stater & Var. (?) & $2.10 \mathrm{~g}$ & $\mathbf{1}$ \\
\hline 23 & $1 / 8-$-stater & Var. 1/8-XIII:01 & $0.87 \mathrm{~g}$ & $\mathbf{1}$ \\
\hline $24-25$ & stater - BIATEC & Var. B-Ia & $6.48-6.47 \mathrm{~g}$ & $\mathbf{2}$ \\
\hline 26 & stater - BIATEC & Var. B-Ic & $6.46 \mathrm{~g}$ & $\mathbf{1}$ \\
\hline 27 & $1 / 3-$ stater - BIAT & Var. 1/3-F/VII:02 & $2.14 \mathrm{~g}$ & $\mathbf{1}$ \\
\hline $28-29$ & $1 / 8-$-stater - BIAT & Var. 1/8-XIV:01 & $0.83-0.80 \mathrm{~g}$ & $\mathbf{2}$ \\
\hline & & & & \\
\hline
\end{tabular}

Tab. 3. Deutsch Jahrndorf. An overview of gold denominations in the hoard. Tab. 3. Deutsch Jahrndorf. Prehled zlatých nominálü zastoupených v depotu.

companied 30 tetradrachms of the Biatec group and probably three hemidrachms of the type Simmering. ${ }^{40}$ Apart from hoards, gold denominations are documented either as isolated finds or entirely lack find circumstances. Moreover, they have not been synthetically published so far. Even so, it can be confidently said that anepigraphic coins clearly prevail over inscribed specimens.

Anepigraphic staters are a particularly typologically complex group among the gold coins minted in Bratislava. Their largest typological variation can be found in the Deutsch Jahrndorf hoard in which 18 specimens have been found. On the other hand, only two examples were represented in the Bratislava 2009 hoard $(\mathrm{Mu}$ silová - Kolníková - Hložek 2015, 253-255, Fig. 13: 14-15). ${ }^{41}$ The typology of the anepigraphic staters is quite complex and therefore, in this paper we limit ourselves to the analysis of specimens from the hoard under discussion. Only a future complex analysis of the topic will make it possible to place these pieces into the full typological variation of these issues. ${ }^{42}$

Parallel production of anepigraphic and inscribed staters is clearly suggested by the presence of both these variations in as many as three hoards and by their identical weight, only rarely exceeding $6.5 \mathrm{~g}$. We can definitely reject the idea that anepigraphic staters represent an earlier phase of coin production in the oppidum of

\footnotetext{
40 Publication of the hoard is in preparation by J. Militký and M. Karwowski.

${ }^{41}$ The gold stater's provenance from the Bratislava 1776 hoard is only an unproved hypothesis (Röttger 2020, 24, Abb. 4).

42 Numerous other specimens were collected e.g. in the unpublished typology of the Biatec group coins (Röttger 2019).
}

Bratislava dated to the late $2^{\text {nd }}$ and early $1^{\text {st }}$ century BC or that they are imports from Bohemia or Moravia (Musilová - Kolníková - Hložek 2015, 273).

Seven groups of anepigraphic staters were distinguished in the Deutsch Jahrndorf hoard (Tab. 3). Their mutual chronological relation nevertheless remains quite unclear. In all cases we are dealing with the shell type with the characteristic bulge on the obverse and a shell-type on the reverse depicting a crescent with rays on the opposite side. This motif is sometimes explained as a solar/lunar symbol and this hypothesis can be further supported by the assumed depiction of lunar phases on the obverse of the variant VI. 1 (cat. no. 17). The term shell-type stater is merely a traditional label with no relation to what the reverse motif actually represents.

In the hoard in question, the stater group $\mathbf{I}$ is represented by three variants, of each of which a single piece is present (cat. nos. 1-3; Tab. 3: 1-3; Fig. 4a: 1-3). The common feature of their obverses is an oval dimple with a hint of a bird head on the smooth bulge and the "shellmotif on the obverse. In variant I.3, however, the reverse is smooth, without the rays over a crescent; this coin is also extremely light weighing a mere $6.18 \mathrm{~g}$.

In the case of the stater group II, four variants were distinguished in the studied hoard, each of which is documented with one to three specimens (cat. nos. 4-10; Tab. 3: 4-10; Fig. 4a: 4-10). The variants represented by several exemplars were clearly minted with the same pairs of dies. The common feature of this group is the characteristic arrangement of the shell motif on the reverse in which the rays above the crescent converge symmetrically to a wedge-shaped 'arrow' in their middle. In variant II.2-4 there are moreover small irregular pellets on the rays. The reverse with the 'wedge-shaped' arrow is characteristic of the shell-staters produced in the Stradonice oppidum, although the motif of the wedge-shaped arrow in the ray field also appears in the latest Bohemian staters with weights ranging from 6.69 to $6.44 \mathrm{~g}$, such as those from the Podmokly hoard and from the Stradonice oppidum (Paulsen 1933, Taf. 19: 394-399; Castelin 1965, 15, var. C 14; Militký 2015a, 202-203, no. 75). In this very specific Bohemian issue both the obverse and reverse motifs (including the dimple on the obverse bulge) clearly recall the Bratislava examples and thus they very probably came into existence in roughly the same period. It is therefore a hint at the synchronisation of the latest phases of coin production in Stradonice with the Bratislava oppidum (Militký 2015a, 49).

The obverse motifs of the stater group II are not identical - in variant II.1 (cat. no. 4; Tab. 3: 4) and II.2 (cat. nos. 5-6; Tab. 3: 5-6) there is a dimple on the smooth bulge. The obverses with dimples in smooth bulges have curious analogies in the finds from the oppidum of Stradonice (Militký 2015a, 200-203, nos. 69-74) although they were surely produced earlier there, specifically in Castelin's phase C (Castelin 1965, 15, var. C 14). As already mentioned, obverse dimples are also present in the latest Bohemian staters with the reverse 'arrow' motif such as those from the Podmokly hoard and the Stradonice oppidum (Paulsen 1933, Taf. 19: 394-399; Castelin 1965, 15, var. C 14; Militký 2015a, 
202-203, no. 75). These issues on the other hand surely came into existence at the same time as the Bratislava pieces with dimples on the obverse bulges (var. II.1-2).

A curious variant is II.3 within the stater group II is documented by three specimens (cat. nos. 7-9; Tab. 3: 7-9). A stylised bird head (?) is represented on the smooth bulge. One exemplar of this variant is also represented in the Bratislava 2009 hoard (Musilová - Kolníková - Hložek 2015, 254-255, no. 15, Fig. 13: 15). This very important type thus creates a link between both hoards. In the last variant II.4 (cat. no. 10; Tab. 3: 10) the obverse bulge is smooth. Variant II.2 is represented also in the hoard from Stupné in Slovakia (Fröhlich 2016, 6-7, no. 5, Fig. 3: 5) in which it was accompanied by two Púchov culture jewels, one spade-type stater, and another stater of the shell-type as well as a 1/3-stater of the shell series (Fröhlich 2016, 5-7, Fig. 3).

The stater group III is represented by a single coin in the hoard (cat. no. 11; Tab. 3: 11; Fig. 4b: 11). On the obverse, there is a smooth bulge with a stylised hand on its upper part. On the reverse there is the "shellmotif with barely visible rays converging towards a central dimple. With its weight of $6.63 \mathrm{~g}$ this is the heaviest of the Deutsch Jahrndorf staters and we can wonder whether it is also the earliest of them. Its image is very similar to the staters with a hand depiction on the obverse from the Stradonice oppidum (Militký 2015a, 197201, nos. 57-68) which are, however, earlier, dating to the phase C of Castelin's relative-chronological scheme (Castelin 1965, 15, var. C 13). The Deutsch Jahrndorf coin must be later than the Stradonice pieces.

Three variants can be distinguished in the stater group IV within the Deutsch Jahrndorf hoard. Each of them is documented by one or two pieces (cat. nos. 1215; Tab. 3: 12-15; Fig. 4b: 12-15); the specimens of the variant IV.2 (cat. nos. 13-14; Tab. 3: 13-14) were probably not minted with the same pair of dies. On the obverses of this group there is a smooth bulge with a stylised hand in its upper part and a prominent pellet below. On the reverses, there are 'shell-motifs' with pronounced rays converging towards a dimple in the centre. In variant IV.3 (cat. no. 15; Tab. 3: 15) there is moreover an oval 'grain' on the right in the rayed field. The issues of the group IV, in particular variant IV.3, have very close analogies among staters from the Stradonice oppidum in Bohemia (Militký 2015a, 202-203, nos. 7678 ). This is an important indicator of their parallel production in the late phases of the Stradonice oppidum which can be synchronised with the existence of the Bratislava oppidum (Militký 2015a, 49). In Bohemia, this very latest Stradonice group (Militký 2015a, 202203, nos. 76-78) follows after the production of staters with the hand motif without a pellet on the smooth obverse bulge (Militký 2015a, 197-201, nos. 57-68). The weight of these issues usually exceeds $7 \mathrm{~g}$ by and large and therefore must be earlier, dating to the phase C of Castelin's relative chronology (Castelin 1965, 15, var. C 13).

There is a single representative of the staters of the group $\mathbf{V}$ in the studied hoard (cat. no. 16; Tab. 3: 16; Fig. 4b: 16). On its obverse, there is a hand at the upper edge of a smooth bulge with three pellets in a horizontal line below. The shell motif on its reverse consists of a crescent and concentrated bunches of rays converging towards the central dimple. On the right side of the field there is an oval 'grain'. Another example of this variant, struck with the same pair of dies is known from the Podmokly hoard (Paulsen 1933, Taf. 18: 387). Therefore, it is open to discussion whether the coin was produced in Bratislava or in Bohemia. In any case it is another important hint at the contemporaneity of the latest phase of the oppida of Stradonice and Bratislava.

Also, the stater of the group VI is represented in the hoard by a single piece (cat. no. 17; Tab. 3: 17; Fig. 4b: 17). On the smooth bulge on the obverse, there is a hand at the upper rim with a pellet between two arches beneath it - it may represent lunar phases. On the reverse, there is again the shell motif consisting of a crescent with the field around the central dimple covered with rays converging towards it. One example of this variant is attested also in the Bratislava 2009 treasure (Musilová - Kolníková - Hložek 2015, 254-255, no. 14, Fig. 13: 14) clearly suggesting a link between the two hoards.

A single coin represents also the group VII (cat. no. 18; Tab. 3: 18; Fig. 4b: 18). On the obverse there is a smooth bulge with a marked pellet in the centre. On the reverse there is again the shell motif with a crescent and the field covered with rays converging towards the central dimple; on the right-hand side of the field there is an oval 'grain'. This variant is attested only in the Deutsch Jahrndorf hoard.

The analysis of the anepigraphic staters from the studied hoard demonstrated that their iconography ties in with Castelin's issues C13-15 (Castelin 1965) produced in Bohemia, in particular in the Stradonice oppidum (Militký 2015a, 196-203, nos. 57-74) in the phase C of Castelin's relative chronology. According to the traditional concept, the production of gold coins ceased in Bohemia at the end of phase $\mathrm{C}$ and shifted to the Bratislava oppidum, whose coinage represents the relative-chronological phase D (Castelin 1965, 15-16, var. D 16-19). The complex study of the coins from Stradonice made it clear, however, that the terminal phases of gold coin production in Bohemia overlap at least partially with the lifetime of the oppidum of Bratislava (Militky 2015a, 49) and the definition of the phases $\mathrm{C}$ and $\mathrm{D}$ therefore requires some revision. As a matter of fact, it is clear that the phase D also includes some Bohemian coins as demonstrated by the pieces from the Podmokly hoard and from the Stradonice oppidum (Paulsen 1933, Taf. 18: 386-387, 19: 394 399; Castelin 1965, 15, var. C 14-15; Militký 2015a, 202-203, nos. 75-78). This is further attested by the pieces from the Deutsch Jahrndorf hoard, in particular by the stater of the group V (cat. no. 16; Tab. 3: 16; Fig. 4b: 16; Paulsen 1933, Taf. 18: 387) in which case it is still hard to decide whether it is originally from Bratislava or in Bohemia.

The analysis of the anepigraphic staters from the Deutsch Jahrndorf hoard shows moreover that these issues were produced over a long period of time in numerous variants. Only a further complex analysis of all the late staters of the relative-chronological phase D may help us understand whether these coins were not also produced elsewhere in the Middle Danube area 
than in Bratislava only. In any case, it is extremely significant that both anepigraphic staters from the Bratislava 2009 hoard find their counterparts in the Deutsch Jahrndorf treasure (var. II.3 and VI.1). It can also be proved beyond any doubt that anepigraphic staters and their subdenominations were minted concurrently with the staters and their subdenominations with the legend BIATEC and NONNOS. We are still not able to account for the numerical prevalence of the anepigraphic coins over the inscribed ones. Nevertheless, the reason could have been a preference for the traditional type without an inscription which could have fulfilled the role of an interregional commercial coin.

Four 1/3-staters of the shell series are represented in the Deutsch Jahrndorf hoard (cat. nos. 19-22; Tab. 3: 19-22). This denomination was studied in detail within the analysis of the coins from the Stradonice oppidum, on which occasion their new typology was outlined (Militký 2015a, 63-69, TAB. 1-4). The denomination was produced throughout the oppida period in Bohemia and probably also in Moravia (Militký 2015a, 49-55, 57). The obverse usually bears a smooth bulge while on the reverse, there is a double smooth bulge framed in a zig-zag line. Six basic types of this denomination, A-F, have been defined (Militký 2015a, 63-69, TAB. 1-4). Their analysis demonstrated that the types A-E were (one after the other) minted in Bohemia; type $\mathrm{E}$ was also minted in Slovakia and the type F exclusively in Bratislava (Militký 2015a, 54, tab. 10). The group E is obviously the latest of the Bohemian production; also, two coins from the hoard of the Trenčianské Bohuslavice oppidum in Slovakia rank within this class (Kolníková 1998, 12-14, Fig. 1: 4-5). The latest group F is already associated only with the Bratislava oppidum phase. It is, however, worth pointing out that the only 1/3-staters of the group F with documented find circumstances are those from the Deutsch Jahrndorf hoard (cat. nos. 20-21; Tab. 3: 20-21); other known specimens lack any information on their provenance (Paulsen 1933, Taf. 23: 475-481).

In the Deutsch Jahrndorf hoard, there is one representative of the 1/3-stater (cat. no. 19; Tab. 3: 19; Fig. $4 b: 19)$ of the variant Militky (2015a) E/VI:02. On its obverse, there is a smooth bulge, on the reverse, there are two smooth facing bulges with a straight base, two horizontal 'grains' below them, with diagonal lines at the bottom and an open zig-zag line around the circumference. Many other examples of this variant are known (Paulsen 1933, Taf. 22: 450-451, 453, 456, 459-461) from the Podmokly hoard and from the Velem-St. Vid oppidum in Hungary. The type E (Militký 2015a, 66, TAB. 4) apparently concludes the long sequence of the numerous variants of the Boii 1/3-staters abundant in Bohemia, in particular in the Stradonice oppidum (Militký 2015a, 216-223, nos. 123-141). Some varieties are also attested in the oppida of Trenčianské Bohuslavice and Velem-St. Vid. The weights of these coins range between 2.3 and $2.2 \mathrm{~g}$. The piece from the Deutsch Jahrndorf hoard represent either the earliest horizon of this denomination produced in Bratislava or it is an import. At the present state of research, we are not able to circumscribe the beginnings of the 1/3-staters production in Bratislava due to the lack of material for comparison.
There are two specimens of 1/3-staters (cat. nos. 2021; Tab. 3: 20-21; Fig. 4b: 20-21) of the variant Militký (2015a) F/VII:01 in the Deutsch Jahrndorf hoard. ${ }^{43}$ Their obverses bear a smooth bulge, on the reverse, there are two smooth facing bulges, two horizontal 'grains' below them, with diagonal lines at the bottom, and an enclosed zig-zag line around the circumference. The same variant of the 1/3-stater is represented also in the hoard from Stupné in Slovakia (Fröhlich 20160, 6-7, no. 6, Fig. 3: 6) together with two Púchov culture jewels, a spadetype stater, and two other shell-type staters (Fröhlich 2016, 5-7, Fig. 3). Several representatives of this variant are attested though without a known provenance (Paulsen 1933, Taf. 23: 475-481). Their weights range from 2.2 to $2.1 \mathrm{~g}$. Another piece found in the hoard discovered in Zuglio Carnico, northern Italy in 1762 , is only documented with an imprecise drawing (Moreno /ed./ 2003, P1 32: I). Unfortunately, also a single example of this type from the Stradonice oppidum is only documented by a poor drawing (Militký 2015a, 222-223, no. 142). These coins - without any doubt the latest of the shell-series 1/3-staters - were probably minted concurrently with the 1/3-staters of the variant Militký (2015a) F/VII:02 with the legend BIAT. As already mentioned, specimens with known find circumstances are attested principally from the hoard under study.

Furthermore, one 1/3-stater with a written legend was present in the Deutsch Jahrndorf hoard (cat. no. 22; Tab. 3: 22). This coin is only known from verbal description without any depiction. Based on its very low weight of $2.10 \mathrm{~g}$ it could have been the variant Militký (2015a) F/VII:01.

Among the gold issues from the Deutsch Jahrndorf hoard there is also one anepigraphic 1/8-stater of the shell series (cat. no. 23; Tab. 3: 23; Fig. 4b: 23). Also, this denomination has been thoroughly studied and its new typology was outlined during the publication of the coin finds from the Stradonice oppidum (Militky 2015a, 7074, TAB. 5-7). The denomination was produced in Bohemia and to some extent probably also in Moravia throughout the entire oppida period (Militký 2015a, 5762). The obverses of these coins usually bear a smooth bulge, while on the reverse there is the motif of a triangle framed with rays and usually with two rows of pellets below. Basically, only one type is attested of which fourteen variants have been distinguished (Militký 2015a, 70-74, TAB. 5-7). Their analysis made it clear that the groups I-XI were minted one after the other in Bohemia, the groups XII-XIV can be located to Slovakia and the oppidum of Velem-St. Vid. A relative chronology of the 1/8-staters has been outlined - the groups XII-XIV are surely the most recent ones (Militký 2015a, 59, TAB. 14). The latter are characterised by the unenclosed triangular motif on the reverse with rays along the sides and without the pellets below. The group XII comes from the oppidum of Trenčianské Bohuslavice (Kolníková 1998, 1218, Fig. 1: 7-12, 2: 1-8). The group XIV is represented by the BIAT inscribed pieces discussed below.

The anepigraphic 1/8-staters associated with the Deutsch Jahrndorf hoard represent the group XIII (Mi-

\footnotetext{
${ }^{43}$ The provenance of the coin cat. no. 21 from the hoard is, however, not certain.
} 
litký 2015a, 74, TAB. 7). Only three exemplars have been preserved (Paulsen 1933, Taf. 29: 706-708), one of which comes from the oppidum of Velem-St.Vid. Another five pieces were present in the Zuglio Carnico 1762 hoard from northern Italy; unfortunately, they are documented only by imprecise drawings (Moreno /ed./ 2003, P1 32: II-VI). Also, in these issues the obverse bears a smooth bulge while on the reverse there is the motif of an unenclosed triangle with rays at the sides and without pellets below. The reverse motif differs slightly from the Bohemian models though still clearly derived from them. The issues of the group XIII can be easily synchronised with the BIAT 1/8-staters of the group XIV: both groups could well have been minted at the same time. The fact that only a single example of the discussed 1/8-staters, variant Militký (2015a) XIII:01 was present in the Deutsch Jahrndorf treasure may reflect a lesser interest in the hoarding of this denomination. 1/8-staters are rather rare also in the Bohemian hoards (Militký 2015a,59). Another reason may be the limited production of 1/8-staters in Bratislava.

The analysis of the anepigraphic stater subdenominations from the Deutsch Jahrndorf treasure proves a direct association of these denominations to the milieu of the latest Bohemian oppida. It is also clear that the oppidum of Bratislava produced much more numerous staters than 1/3- and 1/8-staters. The absence of the lower denominations from the settlement, i.e. as individually lost coins, is particularly eye-catching. In this respect, Bratislava differs notably from the Bohemian oppida (e.g. Stradonice: Militký 2015a, 204-251, nos. 83253) but also from Trančianské Bohuslavice (Kolníková 1998, 12-14, Fig. 1: 4-12). It indicates another role of small gold denominations in the coinage of Bratislava, characterised by the mass-production of the silver Simmering type hemidrachms. The production of 1/3-staters and $1 / 8$-staters could have only symbolically complemented the gold denomination system adopted as a whole from Bohemia, underlining further Bratislava's link with the latest phases of the Bohemian oppida.

The other group of gold issues from the Deutsch Jahrndorf hoard is represented by staters and their subdenominations with inscriptions. The name BIATEC is attested on staters, 1/3-staters, and 1/8-staters while the name NONNOS is so far only known from staters (Musilová - Kolníková - Hložek 2015, 255, Fig. 13: 1013). Most information concerning the coins with the legend BIATEC (or BIAT in the abbreviated form) was until recently known thanks to the Deutsch Jahrndorf hoard; some other examples came from the Parndorf hoard, in the case of another three coins published by $R$. Paulsen (1933, Taf. 29: 695-705, 709-710) the provenance is not known. The views on these coins have been substantially modified by the discovery of the hoard from the Roman structure I on the acropolis of the Bratislava oppidum in which another nine staters with the BIATEC legend have been discovered. The number of these coins have been augmented by a further two recently documented specimens from Slovenský Grob ${ }^{44}$ (Tab. 4: 17; Fig. 7b: 17) and Chorvátsky Grob (Tab. 4: 18). Altogether

\footnotetext{
44 Two other staters with the name BIATEC - not described in more detail - were allegedly present in this find (Musilová - Kolníková - Hložek 2015, 292).
}

18 properly documented examples of staters with the BIATEC legend can be currently listed. Based on the photos of these examples, an entirely new typology has been created (Tab. 4: 1-18; Fig. 7a: 1-11, 7b: 12-17). Three basic types have been distinguished based on the obverse while there are altogether seven varieties produced by six obverse and six reverse dies. ${ }^{45}$

Typological overview of the staters with a legend:

AV stater, shell type - BIATEC, var. B-Ia (dies 1-1); Tab. 4: 1-6; Fig. 7a: 1-6

Obv.: irregular smooth, indistinctly delimited bulge with stylised hand at its upper edge, large pellet on right, beneath hand BIATEC

Rev.: marked crescent at the lower edge, adjacent field covered with marked rays converging towards central dimple, crack in the die on right in rayed field

Ref.: Paulsen 1933, Taf. 29: 696-699; Göbl 1994, Taf. 9: 2-3; Musilová - Kolníková - Hložek 2015, Fig. 13: 2-3; Dembski 1998, Taf. 27: 534-535

Deutsch Jahrndorf, cat. nos. 24-25

AV stater, shell type - BIATEC, var. B-Ib (dies 2-1); Tab. 4: 7; Fig. 7a: 7

Obv.: irregular smooth distinctly delimited bulge with a stylised hand at its upper rim, large pellet with rays on top on right; beneath hand BIATEC (with an oversized C)

Rev.: at the lower rim there is a prominent crescent, adjacent covered with clearly distinct rays converging towards the dimple

Ref.: De La Tour 1892, Pl. LIV: 10165; Paulsen 1933, Taf. 29: 700

AV stater, shell type - BIATEC, var. B-Ic (dies 3-2); Tab. 4: 8; Fig. 7a: 8

Obv.: irregular smooth distinctly delimited bulge, stylised hand at its upper edge, large pellet on right with rays above, beneath the hand BIATEC

Rev.: a marked crescent at the lower edge, adjacent field covered with marked organised rays converging towards central dimple

Ref.: Paulsen 1933, Taf. 29: 695; Castelin 1965, Taf. 3: 31 Deutsch Jahrndorf, cat. no. 26

AV stater, shell type - BIATEC, var. B-IIa (dies 4-3); Tab. 4: 9-10; Fig. 7a: 9-10

Obv.: circular smooth markedly delimited bulge, stylised hand at its upper edge, beneath hand BIATEC Rev.: marked crescent at upper edge, adjacent field covered with marked organised rays converging towards central dimple, crescent in field on right, above marked bulged covered with rays

Ref.: Musilová - Kolníková-Hložek 2015, Fig. 13: 4 and 6

AV stater, shell type - BIATEC, var. B-IIb (dies 5-4); Tab. 4: 11; Fig. 7a: 11

45 Some die combinations were not recognised in the publication of the Bratislava 2009 hoard (Musilová - Kolníková - Hložek 2015, 262, Fig. 15). 


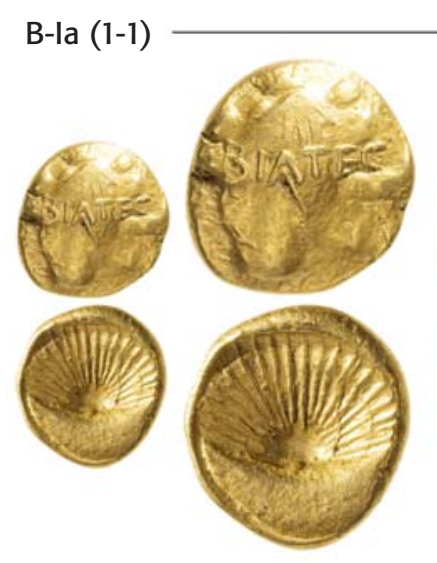

1

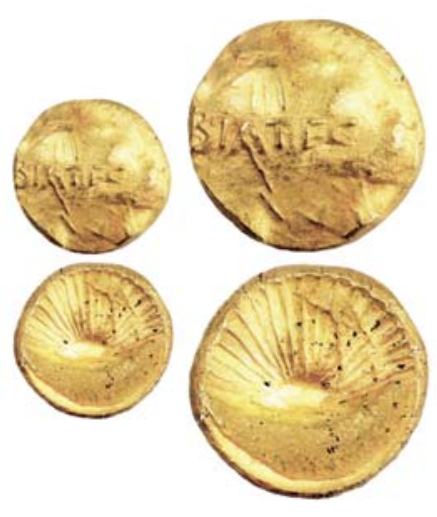

2

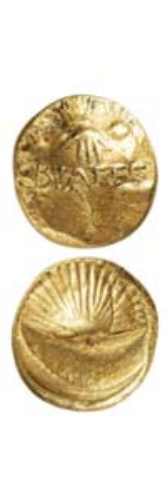

3

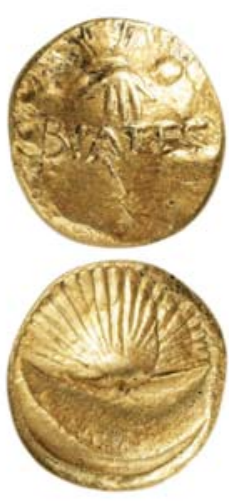

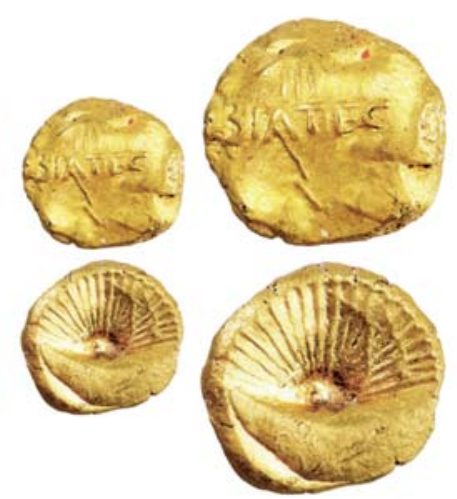

4

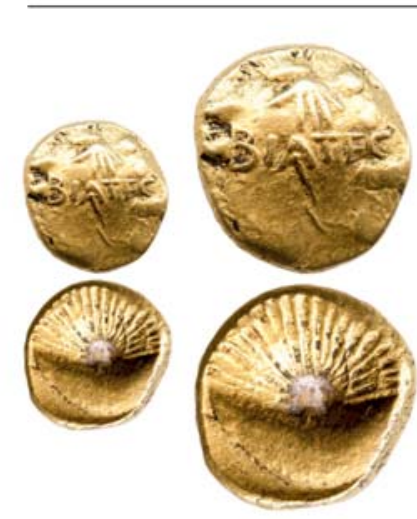

5

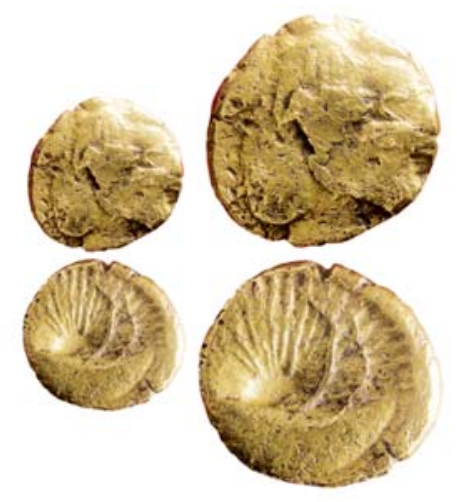

6

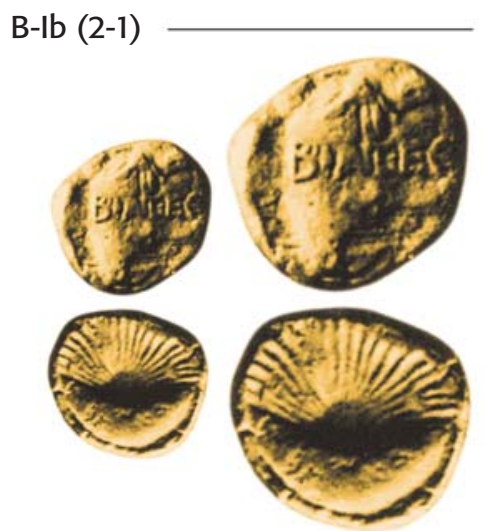

7

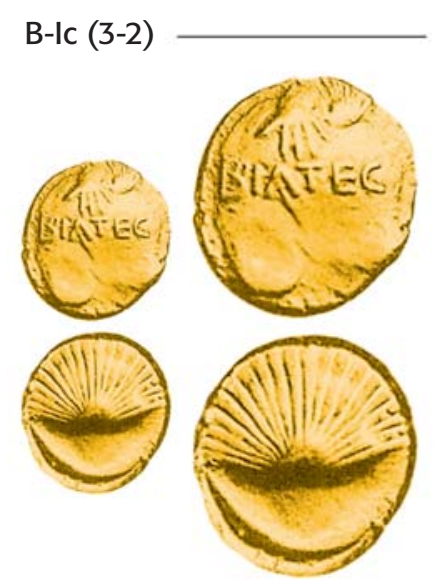

8
B-Ila (4-3)

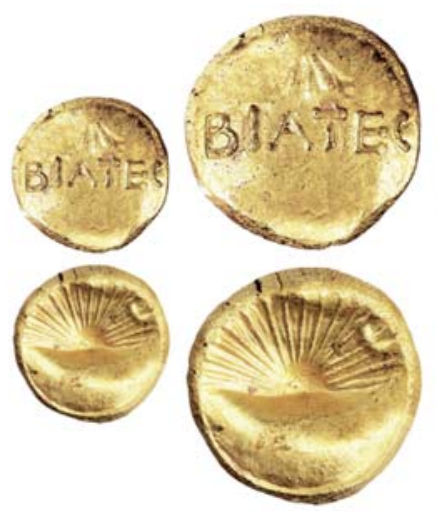

9
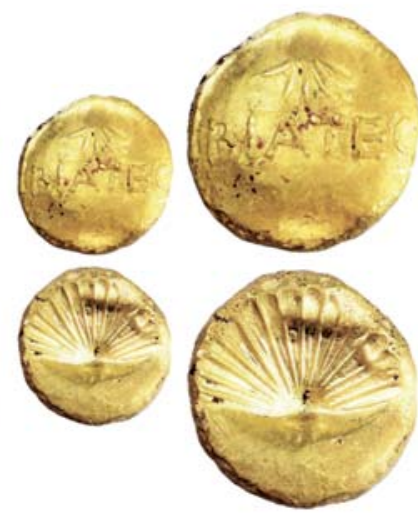

10
B-IIb (5-4)
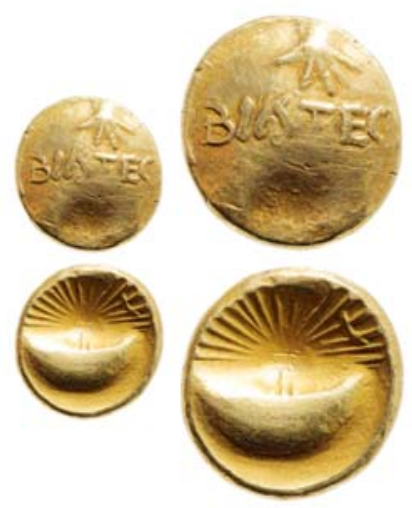

11

Fig. 7a. Typology of the staters with legends BIATEC (the numbering corresponds with that in Tab. 4; 1:1.5; photos by J. Militký, KHM Wien and Röttger 2019). Obr. 7a. Typologie statérů s nápisy BIATEC (Číslování odpovídá tab. 4; 1 : 1,5; foto J. Militký, KHM Vídeň a Röttger 2019).

Obv.: circular smooth undelimited bulge, at its upper edge slightly eccentric stylised hand, beneath BIATEC Rev.: marked crescent at lower edge, adjacent field covered with marked organised rays converging towards the dimple, crescent on right in rayed field
Ref.: Paulsen 1933, Taf. 29: 701; Dembski 1998, Taf. 27: 537

AV stater, shell type - BIATEC, var. B-IIIa (dies 6-5); Tab. 4: 12-16; Fig. $7 b: 12-16$ 
B-IIla (6-5)

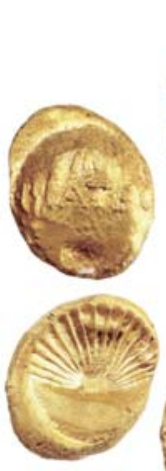

12
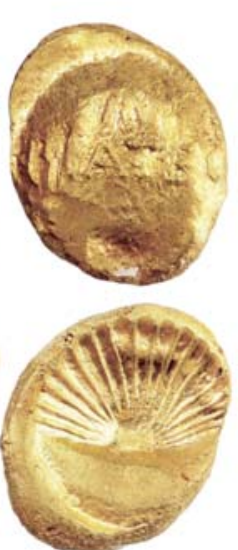

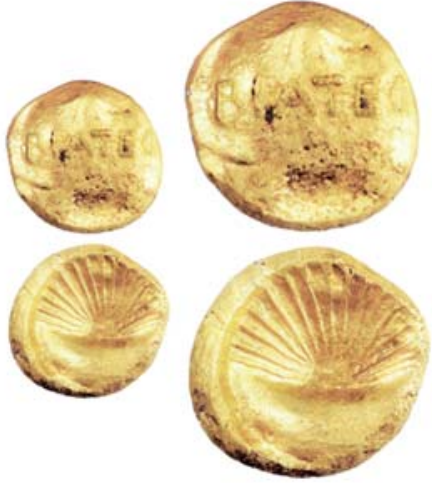

13

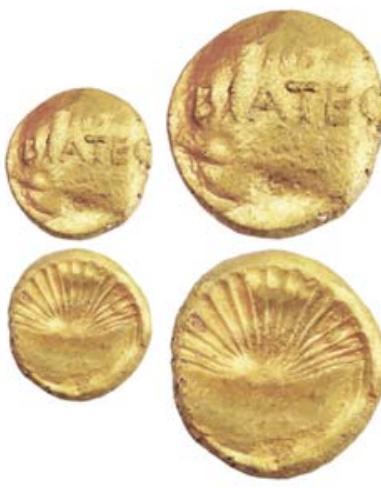

14
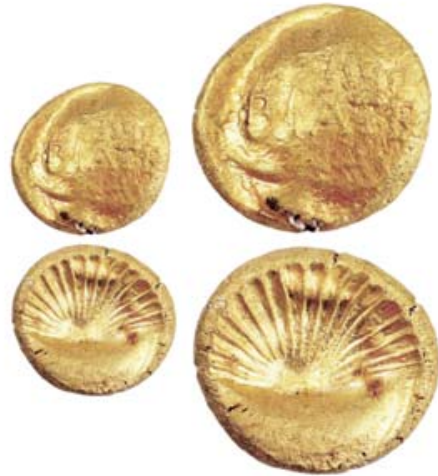

15
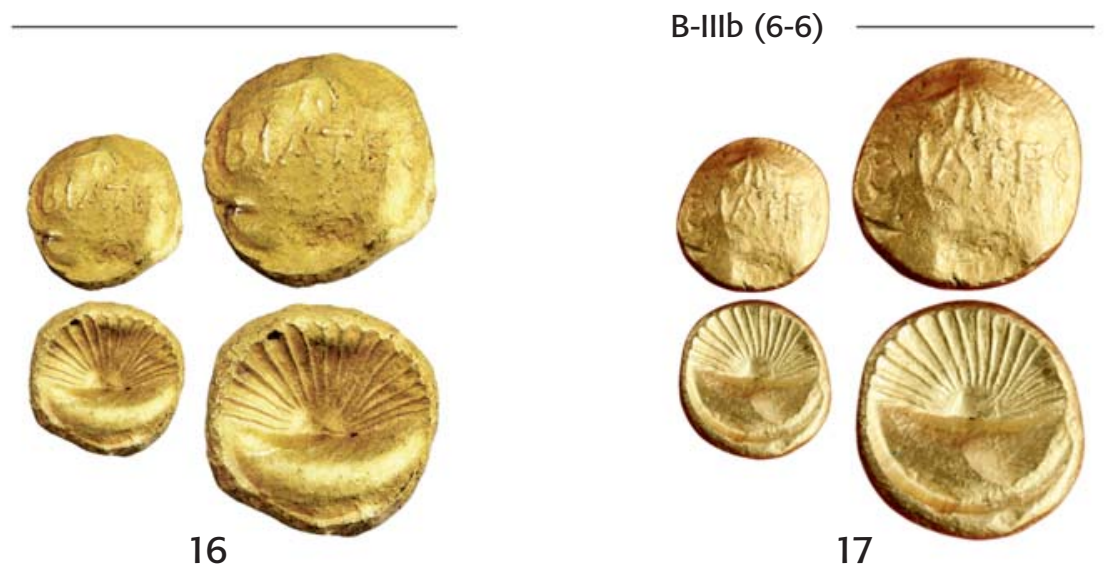

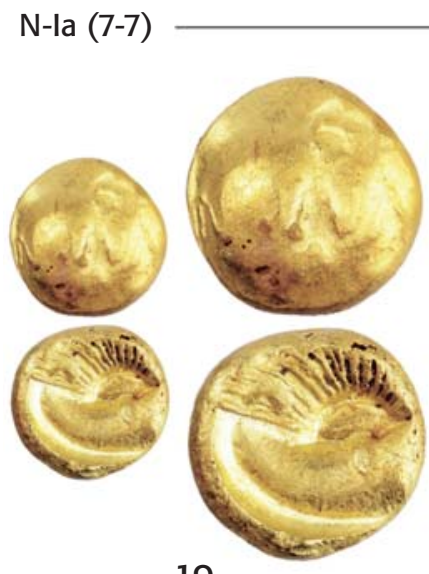

19

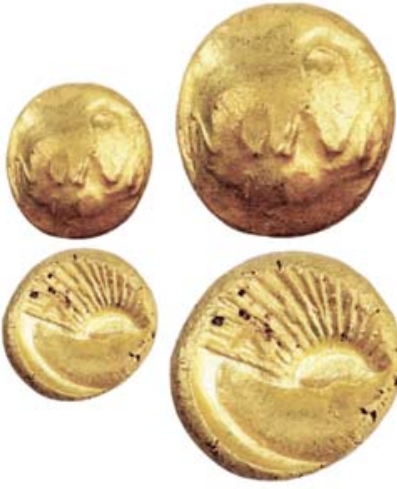

20

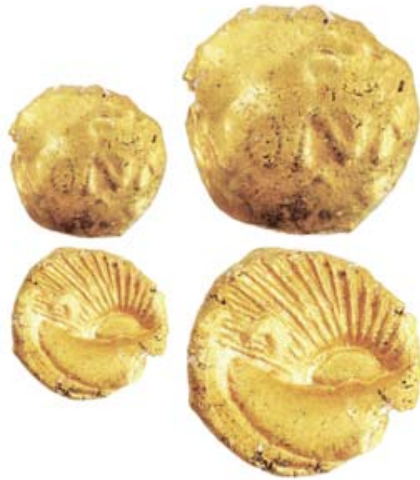

21

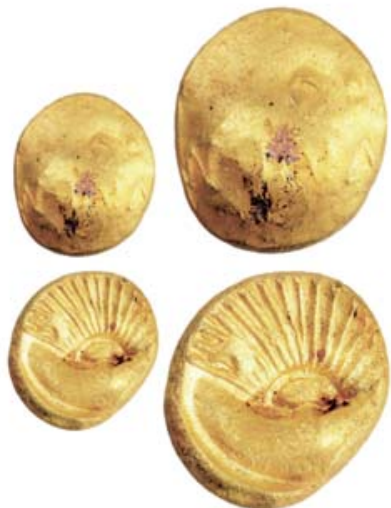

22

Fig. 7b. Typology of the staters with legends BIATEC a NONNOS (the numbering corresponds with that in Tab 4; 1:1.5; photos by J. Militky, KHM Wien and Röttger 2019). - Obr. 7b. Typologie statérů s nápisy BIATEC a NONNOS (číslování odpovídá tab. 4; 1 : 1,5; foto J. Militký, KHM Vídeň a Röttger 2019).

Obv.: irregular smooth markedly delimited bulge, stylised hand at its upper edge, beneath hand BIATEC, roughened bulge surface

Rev.: marked crescent at lower edge, adjacent field covered with marked organised rays converging towards central dimple, crescent in field on right, above bulge covered with rays
Ref.: Musilová - Kolníková - Hložek 2015, Fig. 13: 1, 5, 7-9

AV stater, shell type - BIATEC, var. B-IIIb (dies 6-6); Tab. 4: 17; Fig. 7b: 17

Obv.: irregular smooth markedly delimited bulge, stylised hand at its upper edge, beneath hand BIATEC, roughened bulge surface 
Rev.: marked crescent at lower edge, adjacent field covered with marked organised rays converging towards central dimple

Ref.: Röttger 2015, no. 47-7 / 2288

AV stater, shell type - NONNOS, var. N-Ia (dies 7-7); Tab. 4: 19-22; Fig. 7b: 19-22

Obv.: circular smooth undelimited bulge, in field NONN, above $\mathrm{O}$, beneath $\mathrm{S}$

Rev.: marked crescent at lower edge, adjecent field covered with marked organised rays converging towards central dimple, oval bulge inside the dimple

Ref.: Musilová - Kolníková - Hložek 2015, Fig. 13: 10-13

The staters with the legends BIATEC produced concurrently with the anepigraphic issues rank among the shell type series, bearing a smooth bulge on the obverse and the shell motif on the reverse. Unlike in the anepigraphic varieties, the obverse bulge bears the name BIATEC.

The degree of variety of the BIATEC staters is stunning, in particular when considering the low number of the documented examples; it can be expected that future discoveries of gold coins will bring new variants and die combinations to light. On the other hand, the variability of the staters with the legend BIATEC reflects to some extent the variability of the tetradrachms bearing the same name. The key question is, whether they were minted over a single time-span or repeatedly on various occasions. The motley typology and the testimony of silver denominations plead in favour of the latter option as more probable. Unfortunately, it is still not possible to synchronise the individual staters with specific variants of tetradrachms. The overview in Tab. 4 makes clear the difference in the composition of the hoards Deutsch Jahrndorf and Bratislava 2009. While in the former there are only examples of the variant Ia (cat. nos. 24-25; Tab. 3: 24-25; Fig. 4b: 24-25) and Ic (cat. nos. 26; Tab. 3: 26; Fig. 4b: 26), ${ }^{46}$ the latter included three newly defined variants. It is unclear whether this difference is caused by the diverging chronology of both finds or if there were other reasons at play.

The metrology does not help much in our understanding of the inner chronology of these issues. The absolute majority of the staters range between 6.5 and $6.4 \mathrm{~g}$ (none of them exceeds $6.5 \mathrm{~g}$ ) and the group as a whole is extremely coherent. The SEM-EDX analysis of the alloy composition was carried out only on the specimens from the Bratislava 2009 treasure revealing the gold fineness to be in the range of $88-84 \%$ (Musilová - Kolníková - Hložek 2015, 283-284, Tab. 7-8).

In order to sketch as complete a typology of the Bratislava gold inscribed issues as possible we may also mention the four staters with the legend NONNOS, attested so far exclusively from the Bratislava 2009 hoard (Tab. 4: 19-22; Fig. 7b: 19-22). This new stater type was

\footnotetext{
${ }^{46}$ Also, the coin from MNM Budapest (Paulsen 1933, Taf. 29: 699; Tab. 4: 5; Fig. 7: 5), acquired in 1871, most probably comes from the Deutsch Jahrndorf hoard, though there is no way of proving it. In any case it can be classified as type I represented in the hoard also by other coins.
}

minted with a single pair of dies. On the obverse there is a circular smooth non-delimited bulge with the legend NONN in the field, $\mathrm{O}$ above, and $\mathrm{S}$ below making an unusual word division. The reverse bears a typical shell motif with an unusual oval bulge inside the dimple. The weight of the NONNOS inscribed staters is less than $6.5 \mathrm{~g}$ and the SEM-EDX analyses of their alloy composition showed a gold fineness of 92-83\% (Musilová Kolníková - Hložek 2015, 283-284, Tab. 7-8). Furthermore, the gold staters bearing names also include the somewhat obscure piece with the counter-stamped (?) name DEVIL (Fröhlich 2011, 3-4) whose origin in Bratislava cannot be proved beyond any doubt. ${ }^{47}$

The abbreviated name Biatec can also be found on the 1/3-staters of the shell series with the legend BIAT. From an iconographical point of view these coins carry on the tradition of the issues produced throughout the oppida period in Bohemia and to a certain extent also in Moravia (Militký 2015a, 63-66, TAB. 1-4). On their obverse there is a smooth bulge with an abbreviated variant of the name and on the reverse, there are two oval bulges framed by a zig-zag line. Only a few specimens of the 1/3-staters with the BIAT legend are documented so far; one of them however was present in the Deutsch Jahrndorf hoard (cat. no. 27; Tab. 3: 27; Fig. 4b: 27; Paulsen 1933, Taf. 29: 704). Another famous coin does not in reality come from the treasure, although the contrary was believed for some time (Paulsen 1933, Taf. 29: 705; Militký 2020, Abb. 9.9: 7; Fig. 3: 1). The provenance is unknown also in the case of pieces from the Stockholm coin cabinet (Allen 1972, Taf. IV: 50) and from a private collection (Röttger 2019, Database no. 44-6 / 2296). The coin can be classified as the type F/VII:02 of the new typology of this denomination (Militky 2015a, 66, TAB. 4) which along with the anepigraphic variety Militký (2015a) F/VII:01 discussed above lie at the very end of the shell series 1/3-stater production sequence (Militký 2015a, 54). Both were very likely minted concurrently. The weight of the BIAT $1 / 3$-staters is very low, ranging from 2.2 to $2.1 \mathrm{~g}$. The very small number of the preserved specimens suggests only a very limited production. As with the BIATEC staters, we are not able to synchronise the 1/3-staters with the Biatec group tetradrachms.

Finally, the gold issues from the Deutsch Jahrndorf hoard also include two 1/8-staters of the shell series with the BIAT legend (cat. nos. 28-29; Tab. 3: 28-29). Within the new typology of 1/8-staters they can be classified to the group XIV which is represented by a single variant (Militký 2015a, 72, TAB. 7), very probably minted in parallel with the above discussed anepigraphic group XIII. The obverses of the $1 / 8$-staters with the BIAT legend bear a smooth bulge with the legend, while on the reverse there is the unenclosed triangular motif with the rays at the sides and without the pellets below. These coins are extremely rare - only two preserved examples are known, both from the Deutsch Jahrndorf hoard (cat. nos. 28-29; Tab. 3: 28-29; Fig. 4c: 28-29). Their weights of $0.83 \mathrm{~g}$ and $0.80 \mathrm{~g}$ correspond

\footnotetext{
47 It cannot be excluded that it is only a period imitation of the shell stater manufactured outside of the Bratislava oppidum.
} 
Tab. 4. Staters with legends BIATEC and NONNOS (DJ = Deutsch Jahrndorf; B $2009=$ Bra tislava 2009). - Tab. 4. Statéry s nápisy BlATEC a NONNOS (DJ = Deutsch Jahrndorf B 2009 = Bratislava 2009)

\begin{tabular}{|c|c|c|c|c|c|}
\hline & & Var. & G & Ref. / cat. no. & Hoard / find \\
\hline 1 & \multirow{6}{*}{ BIATEC } & B-la:1 & 6.48 & Cat. no. 24 & DJ \\
\hline 2 & & B-la:2 & 6.468 & Musilová - Kolníková - Hložek 2015, Fig. 13: 2 & B 2009 \\
\hline 3 & & B-la:3 & 6.47 & Cat. no. 25 & DJ \\
\hline 4 & & B-la:4 & 6.458 & Musilová - Kolníková - Hložek 2015, Fig. 13: 3 & B 2009 \\
\hline 5 & & B-la:5 & 6.456 & Paulsen 1933, Taf. 29: 699 & $?$ \\
\hline 6 & & B-la:6 & 6.42 & Paulsen 1933, Taf. 29: 698 & Parndorf \\
\hline 7 & BIATEC & B-lb:1 & 6.43 & Paulsen 1933, Taf. 29: 700 & $?$ \\
\hline 8 & BIATEC & B-Ic:1 & 6.46 & Cat. no. 26 & DJ \\
\hline 9 & \multirow{2}{*}{ BIATEC } & B-Ila:1 & 6.468 & Musilová - Kolníková - Hložek 2015, Fig. 13: 6 & B 2009 \\
\hline 10 & & B-Ila:2 & 6.419 & Musilová - Kolníková - Hložek 2015, Fig. 13: 4 & В 2009 \\
\hline 11 & BIATEC & B-IIb:1 & 6.436 & Paulsen 1933, Taf. 29:701 & $?$ \\
\hline 12 & \multirow{5}{*}{ BIATEC } & B-IIla:1 & 6.465 & Musilová - Kolníková - Hložek 2015, Fig. 13: 9 & B 2009 \\
\hline 13 & & B-IIla:2 & 6.449 & Musilová - Kolníková - Hložek 2015, Fig. 13: 7 & B 2009 \\
\hline 14 & & B-IIla:3 & 6.432 & Musilová - Kolníková - Hložek 2015, Fig. 13: 8 & B 2009 \\
\hline 15 & & B-IIla:4 & 6.428 & Musilová - Kolníková - Hložek 2015, Fig. 13: 5 & B 2009 \\
\hline 16 & & B-IIla:5 & 6.397 & Musilová - Kolníková - Hložek 2015, Fig. 13: 1 & B 2009 \\
\hline 17 & BIATEC & B-IIIb:1 & 6.407 & Röttger 2019, no. 47-7 / 2288 & Slovenský Grob \\
\hline 18 & BIATEC & $?$ & 6.434 & Musilová - Kolníková - Hložek 2015, 292 & Chorvátsky Grob \\
\hline 19 & \multirow{4}{*}{ NONNOS } & N-la:1 & 6.491 & Musilová - Kolníková - Hložek 2015, Fig. 13: 10 & B 2009 \\
\hline 20 & & N-la:2 & 6.477 & Musilová - Kolníková - Hložek 2015, Fig. 13: 11 & B 2009 \\
\hline 21 & & N-la:3 & 6.458 & Musilová - Kolníková - Hložek 2015, Fig. 13: 12 & B 2009 \\
\hline 22 & & N-la:4 & 6.454 & Musilová - Kolníková - Hložek 2015, Fig. 13: 13 & B 2009 \\
\hline
\end{tabular}

with the weights of pieces from Bohemia. The weight of this denomination did not become lower over the course of LT D. Another specimen was found in the Zuglio Carnico hoard discovered in northern Italy in 1762, though it is documented only by an imprecise drawing (Moreno /ed./ 2003, Pl 32: VII). As in the case of the anepigraphic 1/8-staters, it seems probable that (unlike in Bohemia) these coins were produced only in small quantities and probably only had a marginal role in the Bratislava coinage where their role was taken over by the mass-produced Simmering type silver hemidrachms. In this way they rather symbolically complemented the denomination system adopted from Bohemia.

In summary, gold denomination produced in the Bratislava oppidum - both epigraphic and anepigraphic - clearly reflect a connection with Bohemia in the latest phases of its oppida period and at least as far as the current numismatic terminology is concerned, they can be classified to the Boii coinages. This kinship was one of the grounds on which the idea of the departure of the Celtic elites from Bohemia to the Middle Danube area was postulated (e.g. Waldhauser 1983, 335-336; Kolníková 1991, 21). Although there is some logic to this hypothesis and there is no way of disproving it, it seems that this departure did not cause a decline in occupation of Bohemia as was sometimes believed. From a numismatic point of view, it is clear that even in the latest phase D of the coin production, gold and silver denominations did not cease to be produced in Bohemia. It is attested by both their identical iconography and by the weight of the gold denominations. This phenomenon is particularly well visible in the case of staters minted in parallel in Bratislava and in Bohemia, in particular in the oppidum of Stradonice (Militký 2015a, 49).

\subsection{Silver coins}

At least 134 tetradrachms of the Biatec group were represented in the Deutsch Jahrndorf hoard (Tab. 5). The original number of these coins cannot be calculated anymore but it must have been much higher. The absolute majority of the documented coins have been preserved or at least depicted in publications. Only 13 coins have been published only by verbal description without a depiction (cat. nos. 22, 76, 82, 86, 94, 154, 157-163). The coins are catalogued and discussed in the text in alphabetical order of their legends; naturally, it does not reflect in any way their chronology which cannot be established with any certainty. The typological classification of the catalogue is based on the die identification system created by R. Göbl (1994) and complemented in some cases by new combinations according to Ch. Röttger (2019). In each variant, the coins are listed in descending order from the heaviest one which, however, does not reflect the gradual wear of the dies.

The first variant of the Biatec group tetradrachms in the hoard is the issue with the legend AINORIX Göbl (1994) I/1-26, represented there by one specimen (cat. no. 30; Fig. 4c: 30). The issue bears a purely Celtic iconography (though the harpy motif is derived from Mediterranean mythology) with, on the obverse, a bust of a youthful male (?) to the left, in front of him a branch with twigs terminated by pellets (mistletoe?), and on the right a wavy twisted line; the entire scene is framed by a border of dots. On the reverse there is the depiction of a standing Harpy to the right with the head turned to the left. On the right, there is a vertical inscription framed by two vertical lines, AINO, continuing below between two lines XIR; the whole is framed in a border of 


\begin{tabular}{|c|c|c|c|c|}
\hline Cat. nos. & Name & Dies & G & Pcs \\
\hline 30 & AINORIX & $1 / 1-26$ & $16.80 \mathrm{~g}$ & 1 \\
\hline $31-36$ & BIATEC & II/1(1)B-1B & $17.15-16.58 \mathrm{~g}$ & 6 \\
\hline $37-46$ & BIATEC & II/1(1)B-1C & $17.22-16.94 \mathrm{~g}$ & 10 \\
\hline $47-49$ & BIATEC & $11 / 1(2 / 1)-2$ & $17.30-17.12 \mathrm{~g}$ & 3 \\
\hline $50-55$ & BIATEC & $11 / 1(2 / 2)-3$ & $17.42-16.85 \mathrm{~g}$ & 6 \\
\hline $56-67$ & BIATEC & $11 / 1(3)-4$ & $17.31-16.92 \mathrm{~g}$ & 12 \\
\hline $68-72$ & BIATEC & II/2(2)B-6B & $17.21-16.83 \mathrm{~g}$ & 5 \\
\hline $73-74$ & BIATEC & | | II/3-12 & $17.12-17.05 \mathrm{~g}$ & 2 \\
\hline 75 & BIATEC & II/4-3 & $17.02 \mathrm{~g}$ & 1 \\
\hline $76-79$ & BIATEC & II/4-7 & $17.30-17.09 \mathrm{~g}$ & 4 \\
\hline $80-81$ & BVSSVMARVS & III/1-24 & $17.15-17.09 \mathrm{~g}$ & 2 \\
\hline $82-86$ & BVSV & IV/1-28 & $17.1-16.28 \mathrm{~g}$ & 5 \\
\hline $87-88$ & COBROVOMARVS & V/1-18 & $17.27-17.11 \mathrm{~g}$ & 2 \\
\hline 89 & COISA & VI/1-21 & $17.17 \mathrm{~g}$ & 1 \\
\hline $90-92$ & COVIOMARVS & VII/1-23 & $17.50-16.79 \mathrm{~g}$ & 3 \\
\hline $93-95$ & DEVIL (or DEVII) & $\mid X / 1-16$ & $17.26-17.01 \mathrm{~g}$ & 3 \\
\hline $96-98$ & DEVIL (or DEVII) & $I X / 1-20$ & $17.07-17.00 \mathrm{~g}$ & 3 \\
\hline 99 & EVOIVRIX (or FAPIARIX) & $x / 2-19$ & $16.54 \mathrm{~g}$ & 1 \\
\hline $100-103$ & LATVMARVS (IANTVMARVS) & XII/1-14 & $17.02-16.89 \mathrm{~g}$ & 4 \\
\hline $104-121$ & NONNOS & XIV/1A-8 & $17.47-16.85 \mathrm{~g}$ & 18 \\
\hline 122-133 & NONNOS & XIV/1B-9 & $17.48-16.47 \mathrm{~g}$ & 12 \\
\hline 134-136 & NONNOS & XIV/1C-10 & $17.24-16.80 \mathrm{~g}$ & 3 \\
\hline 137-139 & NONNOS & XIV/3-11 & $17.23-16.98 \mathrm{~g}$ & 3 \\
\hline $140-153$ & NONNOS (2ОиИои) & XIV/2 (1)-13 & $17.25-16.79 \mathrm{~g}$ & 14 \\
\hline 154 & NONNOS & ? & $17.12 \mathrm{~g}$ & 1 \\
\hline $155-156$ & TITTO & $X V / 1-25$ & $17.08-17.06 \mathrm{~g}$ & 2 \\
\hline $157-163$ & $?$ & $?$ & $?$ & 7 \\
\hline
\end{tabular}

Tab. 5. Deutsch Jahrndorf. The overview of the silver tetradrachmas represented in the hoard. - Tab. 5. Deutsch Jahrndorf. Prehled stríbrných tetradrachem zastoupených $v$ depotu.

dots. The iconography of the AINORIX tetradrachms is basically identical to the TITTO (Göbl 1994, XV/1-25; cat. nos. 155-156) and FARIARIX (or FAPIARIX) ${ }^{48}$ (Göbl 1994, XI/1-27) tetradrachms. All three were apparently produced in roughly the same period. Overall, 25 exemplars of the AINORIX tetradrachms have been listed in the as yet unpublished Röttger's catalogue (Röttger 2019); out of these, 18 are preserved or at least depicted. In the largest tetradrachm hoard from Bratislava (1942) this issue was represented by two pieces (Ondrouch 1958, nos. 246 and 270). The specimen from the Deutsch Jahrndorf hoard weighs $16.80 \mathrm{~g}$.

The tetradrachms with the legend BIATEC represent the second most numerous group (49 pcs) in the Deutsch Jahrndorf hoard (cat. nos. 31-79). Most die combinations are represented (Tab. 6), including - presented for the first time - new variants within R. Göbl's die combinations II/1(1)-1 and II/2 (2)-6 (Göbl 1994).

The issues Göbl (1994) II/1(1)-1 are very numerous in the hoard. Röttger (2019) defined three new variants of this issue. The first of them, II/1(1)A-1A, is so far represented by a single specimen with an unknown provenance (Fig. 8: 1). The other two variants, II/1(1)B-1B

\footnotetext{
${ }^{48}$ Ch. Röttger $(2013,37)$ proposed an alternative reading of this variant's legend as FAPIARIX.
}

and II/1(1)B-1C, are very well represented in the hoard. R. Göbl considered these two clearly distinct die combinations to be simply the result of die wear (Göbl 1994, Taf. 2: II/1 (1)-1:1-4). This is quite stunning since already $R$. Paulsen clearly distinguished two distinct groups (Paulsen 1933, Taf. 30: 718-721 and Taf. 30: 723-730, 31: 731). The detailed description of the differences can be found in the analysis of each of the variants and it is also obvious from the well-preserved examples (Fig. 8: 2-4).

In the Deutsch Jahrndorf hoard, there are six pieces of tetradrachms with the BIATEC legend of the newly defined variant Röttger (2019) II/1(1)B-1B (cat. nos. 31-36; Fig. 4c: 31-36). This issue combines the iconography of Roman Republican denarii with specifically Celtic motifs. The obverse bears a double portrait - a male and a female head to the right (Honos and Virtus); above their heads there are V-shaped bunches of lines and below on the right there is a leaf; the whole is framed by a smooth line and arches turned inwards. The smooth line of inwards turned arches and the V-shaped bunches are the principal distinguishing features of this die (Fig. 8: 2). The model of the double portrait on the obverse is the depiction of Honos and Virtus on the Roman Republican denarius of the type $R R C$ no. 403/1 dated to $70 \mathrm{BC}(T a b .2)$. In comparison with the Roman model, some details of the representation are visibly barbarised. On the reverse, there is a depiction of a naked male riding a horse to the right; the rider holds the reins in his left hand; his right arm is bent with his right hand stretched out between his chest and the horse's head and holding a branch leaning on his right shoulder; the twigs of the branch terminate in pellets (mistletoe?). Below in the exergue between two lines there is an inscription BIATEC; in front, behind and below the horse, there are leaves; the whole is framed by a smooth line with arches turned inwards. The main distinguishing feature of the reverse die is the line of the rein, which is continually arched and doubled below the horse's chin, as well as a particular conformation of the branch; also, the figure of the rider is stouter than in the other variant and the contour of his head is different (Fig. 8: 2-4). The horseman figure is depicted in a very realistic way with numerous details; in terms of depiction quality the figure ranks among the masterpieces of the Bratislava die engraving. Altogether, 92 tetradrachms of this variant bearing the legend BIATEC are listed in Röttger's unpublished catalogue (Röttger 2019; Tab. 6); 89 of them are preserved or at least depicted. This variant was represented also in the largest tetradrachm hoard unearthed in Bratislava in 1942 (Ondrouch 1958, nos. 25-28, 30, 40-41). The complete identification of all the representatives of this variant will only be possible after a direct revision of the hoard. ${ }^{49}$ The weights of the pieces from the Deutsch Jahrndorf hoard range between 17.15-16.58 g.

In the Deutsch Jahrndorf hoard, there are 11 pieces of tetradrachms with the legend BIATEC of the newly defined variant Röttger (2019) II/1(1)B-1C (cat. nos. 37-46;

\footnotetext{
49 The relatively low-quality photographs do not make it possible to distinguish the individual types in many cases.
} 
Fig. 8. Tetradrachms with the BIATEC legend: 1 - Röttger (2019) II/1(1)A-1A (unknown find-spot; Database no. 2015); 2 - Röttger (2019) II/1 (1)B-1B (cat. no. 33); 3-4 Röttger (2019) II/1 (1)B-1C (cat. nos. 37-38). - Obr. 8. Tetradrachmy s nápisem BIATEC. 1 - Röttger (2019) II/1(1)A-1A (bez lokality; Databáze č. 2015); 2 - Röttger (2019) II/1 (1)B-1B (kat. Č. 33); 3-4 - Röttger (2019) II/1 (1)B-1C (kat. Č. 37-38).

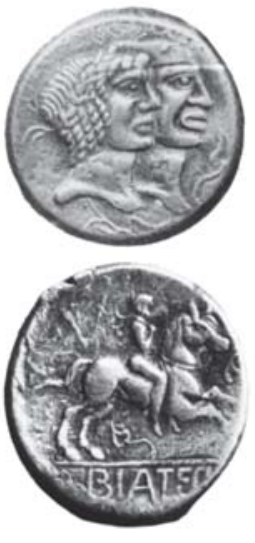

1

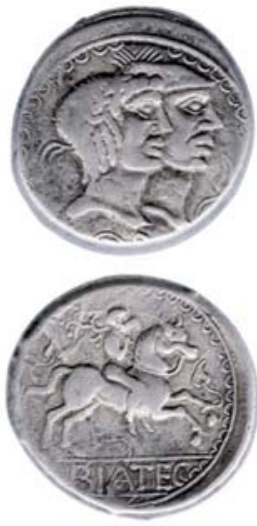

$2(33)$

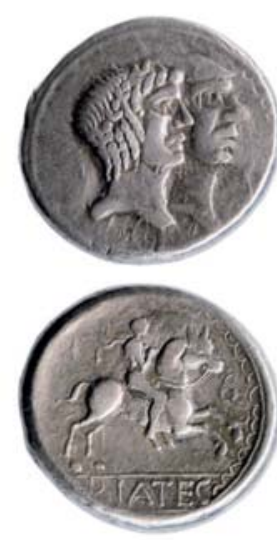

$3(37)$

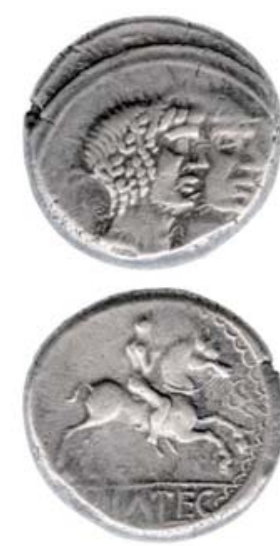

$4(38)$

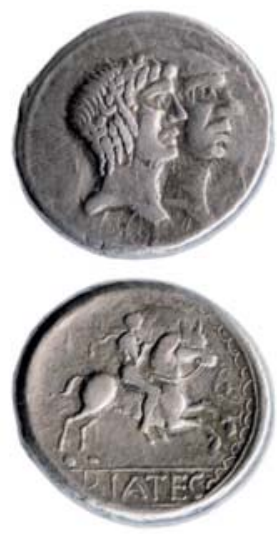

$1(37)$

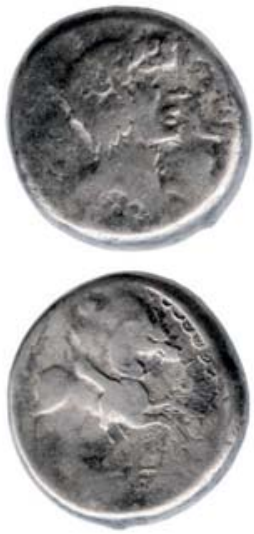

$2(39)$

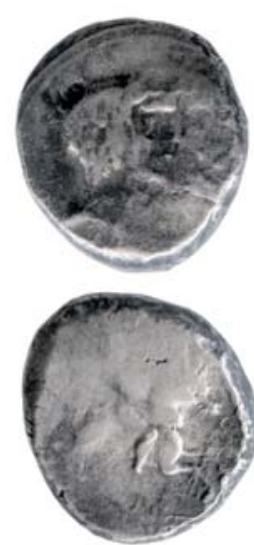

$3(40)$ minted with the previous die that was reworked but with a specimen on its own which was - moreover used over a prolonged period of time which is well attested by its gradual wear (Fig. 9: 1-3). Also, in this case the Honos and Virtus heads are barbarised in some details. The principal distinctive feature of the reverse die is the continually arched rein line below the horse's head and a completely different shape of the branch on the rider's shoulder. Also, the rider figure is overall slenderer and the contour of his head differs from the previous variant (Fig. 8: 2-4). The die combinations Röttger (2019) II/1 (1)B-1B and II/1 (1)B-1C could very likely have been used in roughly the same period. Röttger's unpublished catalogue (Röttger 2019) lists 166 specimens of this tetradrachm variant (Tab. 6), 159 of which are preserved or at least depicted. The variant is also represented in the largest tetradrachm hoard Bratislava 1942 (Ondrouch 1958, nos. 31-34, 43, 47), though the complete identification of all the representatives of this variant will only be possible after a direct revision of the treasure. The weight of the pieces from the Deutsch Jahrndorf hoard range from 17.22-16.94 g.

The tetradrachms with the legend BIATEC, variant Göbl (1994) II/1 (2/1)-2 are represented by three pieces in the Deutsch Jahrndorf hoard (cat. nos. 47-49; Fig. $4 c: 47 ; 4 d: 48-49)$. On the obverse there is again a male and a female head to the right (Honos and Virtus), on the right there is a leaf and the whole is framed with a smooth line with arches turned inwards. Also, in this case the heads of Honos and Virtus are barbarised in
Fig. 9. Examples of gradual die wear of the variant Röttger (2019) II/1 (1)B-1C: 1 - cat. no. 37; 2 - cat. no. 39; 3 - cat. no. 40. - Obr. 9. Príklady postupného opotřebování razidel varianty Röttger (2019) II/1 (1)B-1C: 1 - kat. č. 37; 2 - kat. č. 39; 3 - kat. č. 40 .

some details. The reverse motif is identical to the previous variant though it differs in the rendition of the horseman. The naked rider on a horse to the right holds the rein in his left hand; his right arm is lifted and turned backwards and in the right hand he is holding a branch with leaves and terminating in three pellets (mistletoe?). In the exergue between two lines there is the legend BIATEC and the whole is framed with a smooth line with arches turned inwards. Röttger's unpublished catalogue (Röttger 2019) lists 19 specimens of these tetradrachms (Tab. 6) of which 18 are preserved or depicted. There was only a single exemplar of this variant in the largest tetradrachms hoard discovered in Bratislava in 1942 (Ondrouch 1958, no. 122) which implies its relative rarity. The weight range of the Deutsch Jahrndorf pieces is 17.30-17.12 g.

The Deutsch Jahrndorf hoard further contained six examples (cat. nos. 50-55; Fig. 4d: 50-55) of the tetradrachms with the legend BIATEC var. Göbl (1994) II/1 (2/2)-3. From the iconographic point of view, it is another variation of the Honos and Virtus type. The ob- 


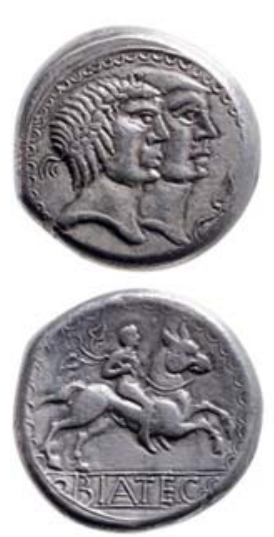

1 (57)
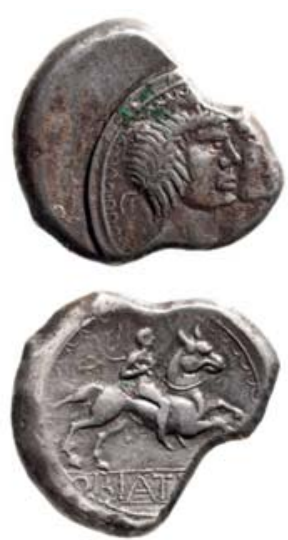

$2(56)$

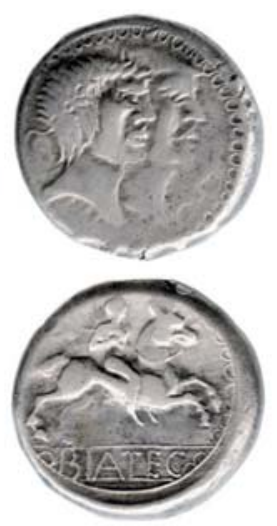

$3(60)$

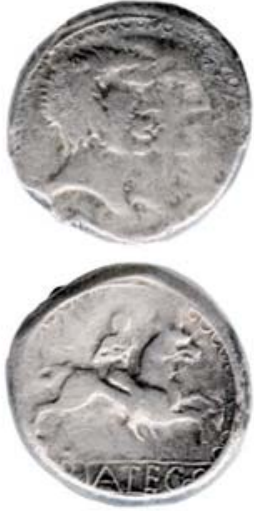

$4(67)$
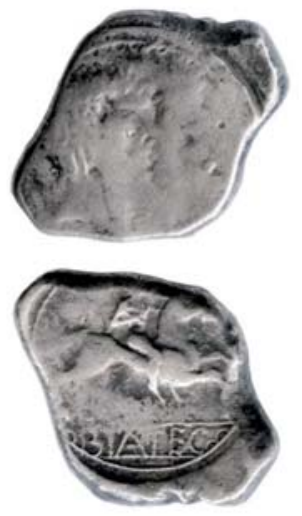

5 (58)
Fig. 10. Gradual wear of the dies of the variant Göbl (1994) 11/1 (3)-4: 1 - cat. no. 57; 2 cat. no. 56; $\mathbf{3}$ - cat. no. $60 ; \mathbf{4}$ - cat. no. 67; 5 - cat. no. 58. - Obr. 10. Prikklady postupného opotřebování razidel varianty Göbl (1994) II/1 (3)-4: 1 - kat. c. $57 ; \mathbf{2}$ - kat. č. 56; $\mathbf{3}$ - kat. c. 60; 4 kat. ̌. $67 ; \mathbf{5}$ - kat. č. 58. verse bears the depiction of a male and a female head to the right (Honos and Virtus), on the right there is a leaf, the whole is framed with a smooth line with arches turned inwards. The heads are rendered in a very rough and barbarised form. The reverse bears the depiction of a naked rider on a horse to the right, holding reins in his left hand and holding in his right hand a branch terminating in two leaves and three pellets (mistletoe?); in the exergue there is the legend BIATEC between two lines; the whole is framed with a smooth line and arches turned inwards. Some pieces were struck with clearly worn dies (cat. nos. 53 and 55). Altogether 94 tetradrachms of this variant are listed in Röttger's unpublished catalogue (Röttger 2019; Tab. 6), out of which 89 are preserved or at least depicted. Also, this type is naturally present in the largest tetradrachms treasure Bratislava 1942 (Ondrouch 1958, nos. 105, 107-109) although the identification of all the specific pieces representing this variant will only be possible after a direct revision of the treasure. The weights of the examples from the Deutsch Jahrndorf hoard range from $17.42-16.84 \mathrm{~g}$.

The Deutsch Jahrndorf hoard also included twelve pieces (cat. nos. 56-67; Fig. 4d: 56-65; 4e: 66-67) of the tetradrachms with the legend BIATEC of the variant Göbl (1994) II/1 (3)-4. Also, this case is a variation of the Honos and Virtus type on the obverse. The heads are complemented with a leaf on the right and the whole is framed with a smooth line and arches turned inwards. Both heads, albeit barbarised, are rendered in a very precise way. On the reverse there is a naked rider on a horse to the right holding the reins in his left hand, in his right hand he holds a branch with three leaves. In the exergue there is the legend BIATEC between two lines and the whole is framed with a smooth line and arches turned inwards. The execution of the reverse die is also extremely fine and the whole is a splendid example of Celtic coin engraving. Both dies must have been very intensely used as is made clear by their gradual wear (Fig. 10: 1-4). In two cases (Fig. 10: 2, 5) the flan is of a completely irregular shape; this is the result of the manufacturing technique - the flans were probably heated before striking and in some cases the temperature may have exceeded the desired limits which caused the uncontrolled expansion of the metal. Röttger's un- published catalogue lists (Röttger 2019) 105 specimens of this tetradrachm variant with the BIATEC legend (Tab. 6), out of which 100 are preserved or depicted. This variant is also represented in the largest tetradrachm treasure Bratislava 1942 (Ondrouch 1958, nos. 67-70, 74-78) although the identification of all the specific pieces representing this variant will only be possible after a direct revision of the treasure. The weights of the examples from the Deutsch Jahrndorf hoard range from 17.31-16.94 g.

The Deutsch Jahrndorf hoard contained five pieces (cat. nos. 68-72; Fig. 4e: 68-72) of the tetradrachms with the BIATEC legend of the newly defined variant Röttger (2019) II/2 (2)B-6B. Also, this case is an iconographic variation of the Honos and Virtus type based on the above-mentioned Roman model. Two sub-variations ( $a$ and $b$ ) have been distinguished, based on the (probably secondary) modification of the letter B in the BIA legend on the obverse (Fig. 11). On the obverse, to the right of the double head there is the inscription BIA and the whole is framed with a smooth line and arches turned inwards. The motif is barbarised as in all the previous types. On the reverse there is the depiction of a naked rider on a horse to the right holding a rein in his left hand and in his right hand a branch terminating in three leaves; in the exergue there is the legend BIATEC between two lines and the whole is framed with a smooth line and arches turned inwards. Röttger's unpublished catalogue (Röttger 2019) lists 86 tetradrachms of this variant (Tab. 6), out of which 83 are preserved or at least depicted. The largest tetradrachms treasure Bratislava 1942 contained four specimens of this variant (Ondrouch 1958, nos. 123-126). The weight range of the Deutsch Jahrndorf hoard pieces is 17.21-16.97 g.

The Deutsch Jahrndorf hoard contained two examples (cat. nos. 73-74; Fig. 4e: 73-74) of the tetradrachms with the legend BIATEC, variant Göbl (1994) II/3-12. Also, in this case the obverse motif is that of Honos and Virtus heads but their rendition differs from the previous types. The double portrait is executed in a specific Celtic stylisation and framed by a beaded line. The origin of the motif in the Roman model ( $R R C$ no. $403 / 1$ ) is not apparent without a knowledge of the other - intermediate - variants. This variant is particularly interesting in its reverse motif. The rider is depicted with 


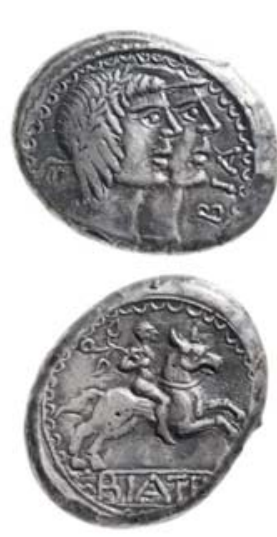

$1(68)$

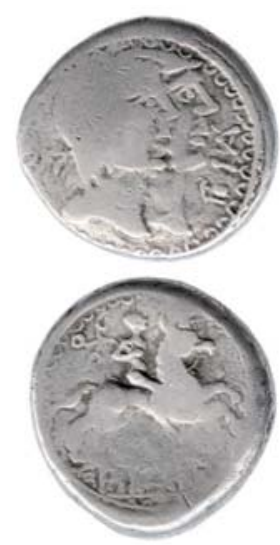

$2(71)$
Fig. 11. Tetradrachms with BIATEC legend: 1 - variant Röttger (2019) II/2 (2)B-6Ba (cat. no. 68); 2 - variant Röttger (2019) II/2 (2)B-6Bb (cat. no. 71). - Obr. 11. Tetradrachmy s nápisem BIATEC: 1 - varianta Röttger (2019) II/2 (2)B-6Ba (kat. c. 68); 2 - varianta Röttger (2019) II/2 (2)B-6Bb (kat. č. 71).

a nude torso (?) but wearing trousers, riding a saddled horse to the right and brandishing a sword in his right hand while in his left hand he holds a large shield (drawn by an oval line around the rider's upper body and the horse's head) and reins (?). In the lower exergue there is the legend BIATEC. Despite the stylisation, the horse is clearly depicted as saddled and also straps for attaching the saddle are clearly represented. This is the only one of the equestrian motifs on the Biatec group coins in which the horse is depicted saddled; the exceptional quality of the representation provides valuable information on the form and use of a shield and sword in mounted combat as well as on the ways of horse saddling (Fig. 12). We can be sure that in this precious iconographic source we see a direct reflection of the period reality (Militký 2013b, 170). The same reverse die was used in a so far unique tetradrachm with the BIATEC legend but with a completely specific obverse (Göbl 1994, Var. II/5-12). A basically identical motif can also be found on a British bronze coin with the legend VER (Röttger 2015, Fig. 7), dated to approximately 25 BC - AD 10 (Cottam et al. 2010, 131, no. ABC 2676). In Ch. Röttger's opinion (Röttger 2015, 201, Fig. 7) this coin confirms the very late chronology of the Biatec coin group. It is, however, possible - and in view of other chronological evidence concerning the Biatec coin group even almost sure - that the Bratislava tetradrachms were rather the model for the British issue. ${ }^{50}$ In other words, the studied tetradrachm is surely earlier than the British issue. Röttger's unpublished catalogue (Röttger 2019) lists 73 specimens of this tetradrachm type (Tab. 6) out of which 66 are preserved or at least

\footnotetext{
${ }^{50}$ Very good examples of the mobility of the Central European Celtic coin iconography to the west are the Treveri quinars of the type 'Eckige Nase' (Delestrée - Tache 2002, 59, no. DT 199, P1. X: DT 199; Depeyrot 2005, 184, no. 96, P1. 3: 96) and South Gaulish obols (Depeyrot 2002, 35, no. 43, P1. 1: 43). Both Gaulish coins imitate the Moravian obols of the type Staré Hradisko D (Militký 2015a, 116-117).
}

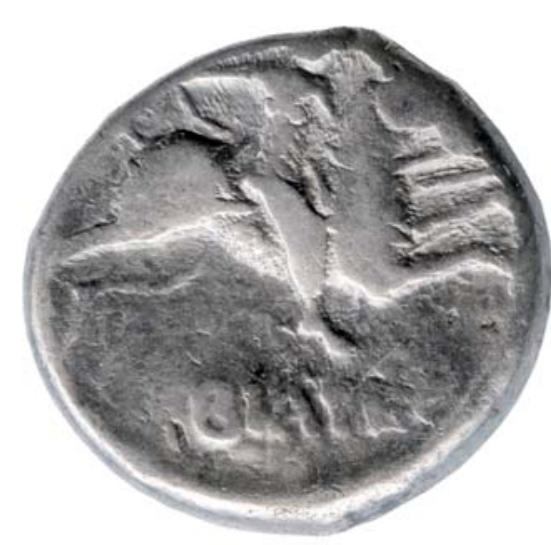

Fig. 12. Tetradrachm with the BIATEC legend, variant Göbl (1994) II/3-12 (cat. no. 74; magnified). - Obr. 12. Tetradrachma s nápisem BIATEC, varianta Göbl (1994) II/3-12 (kat. č. 74; zvětšeno).

depicted. Seven specimens were found in the largest tetradrachm treasure Bratislava 1942 (Ondrouch 1958, nos. 128-134). The weights of the pieces from the Deutsch Jahrndorf treasure range from 17.12-17.05 g.

There is a single specimen (cat. no. 75; Fig. 4e: 75) of a tetradrachm with the BIATEC legend variant Göbl (1994) II/4-3 in the Deutsch Jahrndorf treasure. Its iconography differs from the previously discussed variants with the BIATEC legend - on the obverse there is a male head with short hair to the right framed with a double smooth line and arches turned inwards. The head is depicted in a specifically Celtic style even though also in this case it probably imitates a model taken from the Roman Late Republican coinage; unfortunately, this model cannot be identified. Heads with short-trimmed hair first appeared on the Late Republican denarii before the middle of the $1^{\text {st }}$ century BC (RRC no. 425/1) and became increasingly more common over the third quarter of the first century along with the habit of portraying specific figures. The same portrait type can be found on two tetradrachm types with the legend DEVIL (Göbl 1994, var. IX/1-16 and IX/1-20) and also the bust on one of the types with the NONNOS legend is very similar (Göbl 1994, var. XIV/3-11). The reverse bears the depiction of a naked rider on a horse to the right holding the reins in his left hand while in the right hand he holds a branch terminating in two leaves and three pellets (mistletoe?). In the exergue between two lines there is the legend BIATEC; the whole is framed with a smooth line and arches turned inwards. The same die was used in one of the variants with the Honos and Virtus motif on the obverse (Göbl 1994, var. II/ 1 (2/2)-3) which clearly must have also been struck in the same period as the die combination Göbl (1994) II/4-7 corresponding to the obverse die. The irregular flan shape of the studied variant is probably once again caused by the striking after heating. Seven specimens of this variant of BIATEC inscribed tetradrachms are listed in Röttger's unpublished catalogue (Röttger 2019; Tab. 6). Only one piece was represented in 
the largest tetradrachm treasure from Bratislava 1942 (Ondrouch 1958, no. 6). The specimen from the Deutsch Jahrndorf treasure weighs $17.02 \mathrm{~g}$.

Four coins (cat. nos. 76-79; Fig. 4e: 77-79) in the Deutsch Jahrndorf treasure represent the tetradrachms with BIATEC legend variant Göbl (1994) II/4-7. The iconography of these issues is identical to the previous variant and its obverse is even minted with the same die (Göbl 1994, II/4). The reverse bears the depiction of a naked rider on a horse to the right holding a rein in his right hand and in his left hand a branch terminating with three leaves. In the lower exergue there is the BIATEC inscription between two lines and the whole is framed with a smooth line and arches turned inwards. The reverse motif is the same as in the Honos and Virtus type and the coin was without any doubt minted in the same period as the previous variant Göbl (1994) II/4-3. Altogether, 92 tetradrachms with the BIATEC legend of this variant are listed in Röttger's unpublished catalogue (Röttger 2019; Tab. 6), out of which 87 are preserved or at least depicted. The largest tetradrachm treasure Bratislava 1942 contained 23 pieces (Ondrouch 1958 , nos. 1-5, 7-24). The weight range of the pieces from the Deutsch Jahrndorf hoard is 17.15-17.09 g.

In spite of the extraordinary representation of coins with the BIATEC legend in the hoard, several variants are missing (Göbl 1994, var. II/ 1 (1)A-1A, II/ 1 (4)-5, II/2 (1)-5, II/2 (2)A-6A and II/5-12). These are as a rule very rarely issued, documented each by only single coins; the only exception is var. Göbl (1994) II/ 1 (4)-5 of which 13 pieces are documented (Tab. 6).

The Deutsch Jahrndorf hoard contained two examples (cat. nos. $80-81 ;$ Fig. $4 e: 80-81$ ) of the tetradrachms with the legend BVSSVMARVS of the type Göbl (1994) III/1-24. The iconography of this issue harks back to a Roman model: the obverse bears the image of a male (?) bust to the left with hair tied at the nape; on the left there is a wavy branch (or a feather?) and the whole is framed by a marked beaded line. The motif is based on the representation of Diana in the Roman Republican denarii of the type $R R C$ no. $383 /$ la or $407 / 1$ (Tab. 2). On the reverse there is the image of a gryphon prancing to the right, below in a rectangular frame there is the legend BVSSVM and on the right its continuation A-RV-S; the whole is framed in a smooth line and arches turned inwards. The gryphon image is adopted from the Mediterranean mythology, though the precise iconographic model of this issue cannot be identified. In this way the coin neatly illustrates the free adoption of Mediterranean models and their adaptation to the local Celtic visual style. The tetradrachms with the BVSSVMARVS legend are represented by ten specimens in Röttger's unpublished catalogue (Röttger 2019; Tab. 6); seven of them are preserved or at least illustrated. The largest tetradrachms treasure of Bratislava 1942 contained a single example of this issue (Ondrouch 1958, no. 239). The weight range of the pieces from the Deutsch Jahrndorf treasure is 17.15-17.09 g.

In the Deutsch Jahrndorf treasure, there are five tetradrachms (cat. nos. 82-86; Fig. 4e: 83-85) with the legend BVSV of the variant Göbl (1994) IV/1-28. Also, in this case the iconography is based on Mediterranean models. The obverse again bears the image of a male (?) bust to the left with the hair attached at the nape; on the left there is an S-shaped branch (or feather?), the whole is framed with a marked beaded line. The motif, freely based on the representation of Diana on the Roman Republican denarii of the type RRC no. 383/1a or $407 / 1$ (Tab. 2), is identical to the previous variant but not the die. On the reverse there is the image of a winged centaur (?) to the left with a raised tail, the female half figure is turned back; below, between the centaur's legs, there is the legend BVSV and the whole is framed with a marked beaded line. The precise Mediterranean model of this image is not known and also in this case we are dealing with a unique example of the transformation of the Mediterranean models in the local Celtic style. Röttger's unpublished catalogue (Röttger 2019) lists 64 examples of tetradrachms with the BVSV legend (Tab. 6), 57 of them are preserved or at least published. Seven specimens were found in the largest tetradrachm treasure Bratislava 1942 (Ondrouch 1958, nos. 232-238). The weight range of the pieces from the Deutsch Jahrndorf hoard is 17.1-16.98 g. ${ }^{51}$

The Deutsch Jahrndorf hoard contained two examples (cat. nos. 87-88; Fig. 4f: 87-88) of tetradrachms with the legend COBROVOMARVS of the variant Göbl (1994) V/1-18. On the obverse there is the depiction of a male and a female head to the right (Honos and Virtus or Dioscuri), on the left there is a leaf, on the right there is a star, the whole is framed in a marked beaded line. The motif is adopted from the Roman Republican denarius $R R C$ no. $403 / 1$ dated to $70 \mathrm{BC}$ or $R R C$ no. 463/1a-b (Röttger 2020, 22, Abb. 1) dated to $46 \mathrm{BC}$ $(T a b .2)$ the same as in the case of the tetradrachms with the legend BIATEC. The execution of the double portrait is nevertheless not well mastered in its details and there are marked differences between it and the issues with the BIATEC legend. A clear divergence between the images is the hat rendered with horizontal lines worn by the left figure of the pair. The reverse bears the depiction of a lion prancing to the right; in the exergue between two lines there is the legend $\mathrm{CO}$ BROVOMA, below the lion stomach RV, and above the lion the terminal letter 2 . The image is framed with a smooth line with arches turned inwards. The lion is rendered in a very naturalistic way; the motif is again adopted from the Mediterranean iconography although the exact model is not known. The style of the reverse model is very close to that of the tetradrachms with the legend BVSSVMARVS (Göbl 1994, III/1-24; cat. nos. 8081). Röttger's unpublished catalogue (Röttger 2019) lists 30 specimens of the tetradrachms with the COBROVOMARVS legend (Tab. 6), out of which 25 are preserved or at least depicted. In the Bratislava 1942 tetradrachm treasure, the issue was represented with a single piece (Ondrouch 1958, no. 244). The weight range of the pieces from the Deutsch Jahrndorf hoard is 17.27-17.11 g.

The Deutsch Jahrndorf hoard contained a single tetradrachm (cat. no. 89; Fig. 4f: 89) with the legend

\footnotetext{
51 The weight of coin cat. no. 86 (nowadays lost) is stated as $16.28 \mathrm{~g}$. This extremely low weight is out of the norm for the Biatec tetradrachms group and probably resulted from a flaw in the manufacturing process. At the same time, we cannot exclude an error which occurred during weighing or in publication.
} 
COISA, variant Göbl (1994) VI/1-21. The iconography of this issue combines the Roman model with specifically Celtic inventiveness. The obverse bears a male head in a helmet (or hat) to the left with a drape at the neck; the depiction is framed with a 'chain' line. The face of the figure is stylised in a clearly Celtic style though the model can be identified in Roman Republican denarii $R R C$ no. $381 / 1$ a or no. $401 / 1$ dated to 80 and $71 \mathrm{BC}$ respectively (Tab. 2). The reverse motif is specifically Celtic, depicting a standing male to the right fighting a rampant bear (?) or boar (?) to the left; below in the exergue there is the legend COISA (turned by $180^{\circ}$ ); the whole is framed with a 'chain' line. The animal is depicted somewhat naïvely with all four paws held out towards the human figure and so it seems to be hovering in the air. Röttger's unpublished catalogue (Röttger 2019) lists 20 specimens of the tetradrachms with the COISA legend (Tab. 6), 17 of which are preserved or at least depicted; the issue is therefore very rare. Only a single piece was present in the largest tetradrachm hoard from Bratislava 1942 (Ondrouch 1958, no. 245). The piece from the Deutsch Jahrndorf hoard weighs $17.17 \mathrm{~g}$.

The Deutsch Jahrndorf hoard contained three pieces (cat. nos. 90-92; Fig. 4f: 90-92) of tetradrachms with the legend COVIOMARVS variant Göbl (1994) VII/1-23. The iconography of this coin is again specifically Celtic. The head of a youth to the right on the obverse is framed with a 'spruce wreath'. The motif is almost identical to that in an anepigraphic tetradrachm, variant Göbl (1994) XVI/1-17. It might be loosely inspired by the portrait style of the Roman Republican denarii. The reverse bears a stylised depiction of a standing boar to the left on top of a straight horizontal line with four stylised plants (?); above there is the arched legend COVIOMARVS and the whole is framed in a smooth line. The boar motif may represent an insight into the traditional Celtic mythology. Only seven tetradrachms with the COVIOMARVS legend are listed in Röttger's unpublished catalogue (Röttger 2019; Tab. 6) suggesting the rarity of the issue. Not a single piece of this type was present in the largest tetradrachm hoard from Bratislava 1942 (Ondrouch 1958). The weight range of the pieces from the Deutsch Jahrndorf hoard is 17.50-16.79 g.

The Deutsch Jahrndorf hoard contained three specimens (cat. nos. 93-95; Fig. 4f: 93-95) ${ }^{52}$ of tetradrachms with the legend DEVIL variant Göbl (1994) IX/1-16. ${ }^{53}$ A male head with short hair to the right framed with a beaded line is depicted on the obverse. The head is rendered in a specifically Celtic style; though apparently inspired by Roman Late Republican coinage, the exact model cannot be identified. Heads with short-trimmed hair first appeared on Late Republican denarii before the middle of the $1^{\text {st }}$ century BC (RRC no. 425/1) and became more frequent over the course of the third quarter of the $1^{\text {st }}$ century with the increasing tendency to

\footnotetext{
${ }^{52}$ In the case of the coin cat. no. 94 the provenance from the treasure is not certain.

53 An alternative reading proposed for the legend DEVIL is DEVII (Röttger 2013, 34-36).
}

represent actual portraits on coins. The same portrait type can be found on two types of tetradrachms with the legend BIATEC (Göbl 1994, var. II/4-3 and II/4-7) and also the bust on one of the types with the name NONNOS is very similar (Göbl 1994, var. XIV/3-11). On the reverse, there is the depiction of a prancing horse to the right; above there is the legend DEVIL (the inscription is turned by $180^{\circ}$ ) and the whole is framed with a smooth line with arches turned inwards - the legend is usually not very distinct. The horse figure is rendered in a specifically Celtic style and the figure is slightly stylised in some details (e.g. the head). The flan of one of the coins from the hoard (cat. no. 95) is slightly eccentric with a projecting lug and traces of repeated strikes on the obverse. The lug may have come to be during the pre-heating of the flan or possibly by metal expansion during striking. ${ }^{54}$ Röttger's unpublished catalogue (Röttger 2019) lists 40 specimens of this variant of tetradrachms with the legend DEVIL (Tab. 6), 39 of which are preserved or at least depicted - the issue is therefore relatively rare. Ten specimens of this issue were represented in the largest tetradrachm hoard from Bratislava 1942 (Ondrouch 1958, nos. 216-225). The weight range of the pieces from the Deutsch Jahrndorf hoard is $17.26-17.01 \mathrm{~g}$.

Probably three coins (cat. nos. 96-98; Fig. 4f: 96-98) ${ }^{55}$ in the Deutsch Jahrndorf hoard represent the tetradrachms with the legend DEVIL variant Göbl (1994) IX/1-20. The obverse of these coins is struck with the same die as the previous variant (Göbl 1994, IX/1-16) which suggests the parallel production of both variants. The reverse motif represents a standing bear to the right framed by a smooth line; below in exergue there is the legend DEVIL (or DEVII). ${ }^{56}$ The bear is depicted very realistically including its fur which is well visible in the piece from the hoard Bratislava 1942 (Ondrouch 1958, no. 226). The iconographic origin of this type is not certain though the location of the legend in the exergue harks back to the Roman Republican denarii. Tetradrachms of this variant with the legend devil listed in Röttger's unpublished catalogue (Röttger 2019) number 40 specimens ( $\mathrm{Tab}$. 6), out of which 39 are preserved or at least depicted. The largest tetradrachm hoard of Bratislava 1942 contained 10 specimens of this variant (Ondrouch 1958, nos. 226-231 and 269). The weight range of the specimens from the Deutsch Jahrndorf hoard is $17.07-17.00 \mathrm{~g}$.

The Deutsch Jahrndorf hoard included a single exemplar (cat. no. 99; Fig. 4f: 99) of the tetradrachms with the legend EVOIVRIX ${ }^{57}$ variant Göbl (1994) X/2-19. The obverse bears a depiction of a youth bust to the right, on the right there are four leaves, on the left there

${ }^{54}$ In this case the metal expansion cannot be considered to be certain.

55 The provenance of the coins cat. nos. 96-97 from the hoard is not certain.

${ }^{56}$ An alternative reading proposed for the legend DEVIL is DEVII (Röttger 2013, 34-36).

57 Ch. Röttger (2013, 37-38) proposed reading the legend in both variants (Göbl 1994, var. X/1-19 and X/2-19) in a retrograde way as FAPIARIX. In this study we adhere to the traditional reading EVOIVRIX and this reading can be regarded as more likely. 


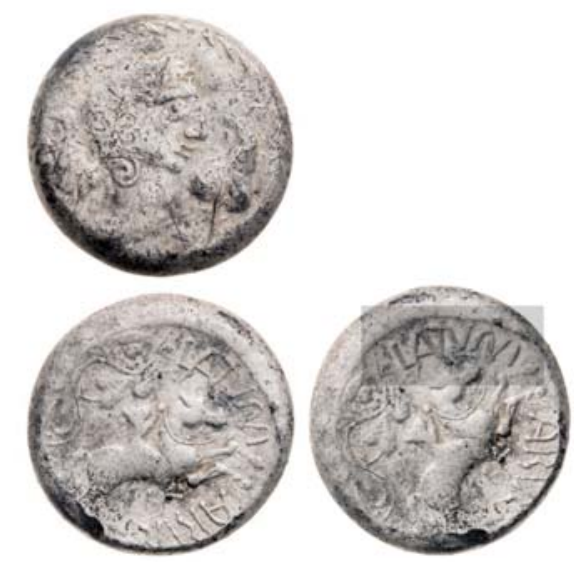

1

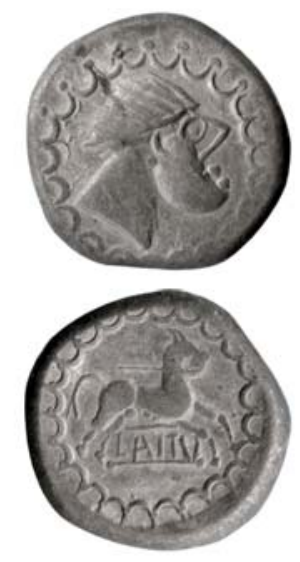

2

Fig. 13. Tetradrachms with the LATVMARVS and LATTV legends: 1 - legend LATVMARVS on the reverse of the piece not originating from the Deutsch Jahrdorf hoard (J. Militky's archive); 2 - teradrachm with the LATTV legend (Militky 2013b, 166, Fig. 1c: 22). - Obr. 13. Tetradrachmy s nápisy LATVMARVS a LATTV: 1 - nápis LATVMARVS na rubu exempláre nepocházejíciho z depotu Deutsch Jahrdorf (archiv J. Militkého); 2 - tetradrachma s nápisem LATTV (Militký 2013b, 166, obr. 1c: 22).

is a monogram $A \bar{A}$. The motif is depicted in the Celtic style but it is loosely based on Roman Republican models. On the reverse, there is a depiction of a lion jumping to the left with a stylised mane composed of triangles; below between two lines there is the legend EVOIVRIX and the whole is framed with a smooth line with small arches turned inwards. Although the lion depiction is strongly stylised and adapted to the Celtic visual style, the motif is clearly inspired by Mediterranean models. Röttger's unpublished catalogue (Röttger 2019) lists 20 specimens of this tetradrachm variant with the legend EVOIVRIX (Tab. 6), out of which 17 are preserved or at least depicted. This coin type was not represented in the largest tetradrachm hoard from Bratislava 1942 (Ondrouch 1958). The piece from the Deutsch Jahrndorf hoard weighs $16.54 \mathrm{~g}$. We can mention in passing that the hoard did not contain any coin of the rare var. EVOIVRIX struck with the same reverse die - Göbl (1994) X/1-19 (Tab. 5).

The Deutsch Jahrndorf hoard contained four specimens (cat. nos. 100-103; Fig. 4f: 100-103) of the tetradrachms with the inscription LATVMARVS (IANTVMARVS) of the type Göbl (1994) XII/1-14. This legend was for a long time being read as IANTVMARVS with ANT in ligature. The detailed study analysis of the hoard however made it absolutely clear that this reading is incorrect. A much simpler reading LATVMARVS is equally possible; the letters LATV are clearly visible on another well struck and well-preserved piece which does not come from the hoard under investigation (Fig. 13: 1). ${ }^{58}$ Moreover, in this way the legend correlates with the recently published new type with the legend LATTV (Militký 2013b, 162, nos. 21-22, Fig. 1c: 21-22; 2014; Fig. 13: 2) ${ }^{59}$ found in a hoard discovered in the surroundings of Salzburg (Militký 2013b; Röttger 2015, 195, 205). It turns out moreover, that both coins are clearly associated with the Bratislava oppidum even though their clearly different style suggests that they

\footnotetext{
58 This fact was first realised by auctioneers who presented this tetradrachm type as LATVMARUS (Macho \& Chlapovič 9, no. 6; Macho \& Chlapovič 10, no. 14; Macho \& Chlapovič 12, no. 8; Macho \& Chlapovič 18, no. 1004).

59 After Röttger (2019) var. XVII/1-31.
}

were not minted at the same time and the LATTV tetradrachm is possibly the latest issue of the Biatec group (Militky 2013b, 173). ${ }^{60}$ We cannot be sure either whether LATVMARVS and LATTV were the same person; the concurrent use of a single and a double $T$ need not necessarily mean that we are dealing with two different characters: the same alternation of a single or double $\mathrm{S}$ can be observed in the names BVSV and BVSVMARVS (cat. nos. 80-86; Fig. 4e: 80-85) which can also apply to a single person. Ch. Röttger (2013) recently proposed new readings of the names DEVIL as DEVII, FARIARIX as FAPIARIX, and EVOIVRIX as a mirrored variation of the name FARIARIX or FAPIARIX. These reinterpretations, no matter how interesting, are not certain. On the contrary, the LATVMARVS legend is clearly legible and - combined with the LATTV legend it is a significant milestone in our understanding of the onomastics on the Bratislava tetradrachms. The iconography of this issue is purely Celtic. The obverse image depicts a youthful bust with curly hair to the right, on either side there is a large leaf, the whole is framed with a 'spruce wreath'. On the reverse there is the image of a dressed rider on a horse to the right holding a rein; above there is a tendril with leaves; around the circumference and below there is the legend LATVMARVS, the final $\mathrm{S}$ being mirror-flipped. The depiction of the rider is basically identical to one of the BIATEC types (Göbl 1994, var. II/ 1 (1)-1) with the sole exception that in this case the rider is not holding a branch in his hand. Röttger's unpublished catalogue (Röttger 2019) lists 46 tetradrachms with the legend LATVMARVS (Tab. 6), 43 of which are preserved or at least depicted. In the largest tetradrachm treasure from Bratislava 1942 the type was represented by four specimens (Ondrouch 1958, nos. 240-243). The weight range of the pieces from the Deutsch Jahrndorf hoard is 17.02-16.89 g.

The most numerous tetradrachms of the Deutsch Jahrndorf hoard (51 pcs) are those bearing the legend NONNOS (cat. nos. 104-154). Basically, all the die com-

\footnotetext{
60 Some doubts about the Bratislava origins of the LATTV coin, which had recently been expressed (Kolníková-Bakos - Pauditš $2018,158)$ are perfectly dispelled by this new reading of the LATVMARVS legend.
} 
binations are present with the exception of the very rare variant Göbl (1994) XIV/1B-10. One NONNOS coin from the hoard is only documented with an imprecise description and cannot be classified with any certainty (cat. no. 154). R. Göbl (1994) believed that the variants $\mathrm{XIV} / 1 \mathrm{~A}-8$, XIV/1B-9, and XIV/1C-10 represent only three subsequent wear phases of a single obverse die. The analysis of the coins from the Deutsch Jahrndorf hoard proved beyond any doubt that in reality they represent three individual dies. The differences in the head depiction are so significant that the idea of a single repeatedly re-worked die is untenable.

18 coins from the Deutsch Jahrndorf hoard (cat. nos. 104-121; Fig. 4f: 104-105, 4g: 106-121) ${ }^{61}$ represent tetradrachms with the NONNOS legend of the variant Göbl (1994) XIV/1A-8. The iconography of this issue combines Roman Republican models with a specifically Celtic style. On the obverse there is a depiction of a female head with a diadem and a complex hairdo to the right; below her neck there is a wavy line and the whole is framed with a dotted border and small arches turned inwards. The head is loosely inspired by the Roman Republican denarius of the type $R R C$ no. $410 / 1$ dated to $66 \mathrm{BC}(T a b .2)$. The reverse bears the depiction of a naked rider on a horse to the right holding a sword in his right hand and reins and a branch in his left hand. In the lower exergue there is the legend NONNOS between two lines and the whole is framed with a smooth line with arches turned inwards. This motif further enlarges the wide array of horseman depictions on the $\mathrm{Bi}$ atec group tetradrachms. With the exception of only a few details, the reverse motif is iconographically identical to the tetradrachms of the variant Göbl (1994) XIV/1B-9, XIV/1B-10, XIV/1C-10, and XIV/3-11. Some pieces from the hoard make it possible to observe the gradual wear of the dies (e.g. cat. nos. 107-108, 112, 116). Ch. Röttger (2019) lists in his unpublished catalogue 110 NONNOS tetradrachms of the variant in question (Tab. 6), 108 of which are preserved or at least depicted. In the largest tetradrachm treasure Bratislava 1942 , this variant was represented by 21 pieces (Ondrouch 1958, nos. 136-137, 139, 141, 143-146, 148$149,154-155,161,164,176-178,268)$. The weight range of the pieces from the Deutsch Jahrndorf treasure is 17.47-16.85 g. The upper value is remarkably high.

The Deutsch Jahrndorf hoard contained 12 examples (cat. nos. 122-133; Fig. 4g: 122-123, 4h: 124-133) of the NONNOS inscribed tetradrachms of the variant Göbl (1994) XIV/1B-9. The iconography of this issue combines Roman Republican models with a specifically Celtic style. On the obverse there is a depiction of a diademed female head with complex hairdo to the right; below her neck there is a wavy line and the whole is framed with dots and small arches turned inwards. The head depiction is basically identical to the previous variant differing only in the hairdo details. The head is loosely inspired by the Roman Republican denarius of the type $R R C$ no. $410 / 1$ dated to $66 \mathrm{BC}$ (Tab. 2). The reverse bears the depiction of a naked rider on a horse to

\footnotetext{
${ }^{61}$ In the case of the coin cat. no. 104 the provenance from the hoard is not certain.
}

the right holding a sword in his right hand and reins and a branch in his left hand. In the lower exergue there is the legend NONNOS between two lines and the whole is framed with a smooth line with arches turned inwards. With the exception of only a few details, the reverse motif is iconographically identical to the tetradrachms of the variant Göbl (1994) XIV/1A-8, XIV/1B$10, \mathrm{XIV} / 1 \mathrm{C}-10$, and XIV/3-11. Some pieces from the hoard make it possible to observe the gradual wear of the dies (e.g. cat. nos. 122-125, 127-128, 131). Ch. Röttger (2019) lists in his unpublished catalogue 122 NONNOS tetradrachms of the variant in question (Tab. 6), out of which 121 are preserved or at least depicted. The largest tetradrachm treasure Bratislava 1942 contained 26 pieces of this variant (Ondrouch 1958, nos. 138, 140, $142,147,150-153,156-160,162-163,165-175)$. The weight range of the pieces from the Deutsch Jahrndorf treasure is $17.48-16.47 \mathrm{~g}$. Both the extreme values are extraordinary and out of the norm.

The Deutsch Jahrndorf hoard contained three pieces (cat. nos. 134-136; Fig. 4h: 134-136) of the NONNOS inscribed tetradrachms of the variant Göbl (1994) XIV/1C-10. Also, in this case the iconography of this issue combines Roman Republican models with a specifically Celtic style. On the obverse there is a depiction of a diademed female head with a complex hairdo to the right; below her neck there is a wavy line and the whole is framed with dots and small arches turned inwards. The head is loosely inspired by the Roman Republican denarius of the type $R R C$ no. $410 / 1$ dated to $66 \mathrm{BC}$ (Tab. 2). The reverse bears the depiction of a naked rider on a horse to the right holding a sword in his right hand and a rein in his left hand. In the lower exergue there is the legend NONNOS between two lines and the whole is framed with a smooth line with arches turned inwards. With the exception of only a few details, the reverse motif is iconographically identical to the tetradrachms of the variant Göbl (1994) XIV/1A-8, XIV/1B-9, XIV/1B-10, and XIV/3-11. Röttger's unpublished catalogue (Röttger 2019) lists 97 NONNOS tetradrachms of the variant in question (Tab. 6), 84 of which are preserved or at least depicted. The largest tetradrachm treasure Bratislava 1942 contained 5 pieces of this variant (Ondrouch 1958, nos. 179-183). The weight range of the pieces from the Deutsch Jahrndorf treasure is 17.24-16.80 g.

Three pieces from the Deutsch Jahrndorf treasure (cat. nos. 137-139; Fig. 4h: 137-139) represent the NONNOS inscribed tetradrachms of the variant Göbl (1994) XIV/3-11. The obverse bears the depiction of a male bust with short hair to the left, before him on the left there is a branch terminating in pellets (mistletoe?), the whole is framed with a line of marked dots. The head is rendered in a specifically Celtic style although it is probably inspired by a Roman Late Republican model whose precise identification is not possible. Heads with short-trimmed hair first appeared on Late Republican denarii before the middle of the $1^{\text {st }}$ century BC (RRC no. 425/1) and became common in the third quarter of the $1^{\text {st }}$ century with the generalisation of the habit of depicting portraits of specific personalities. The same portrait type can be found in two types of tetradrachms with the legend BIATEC (Göbl 1994, var. 


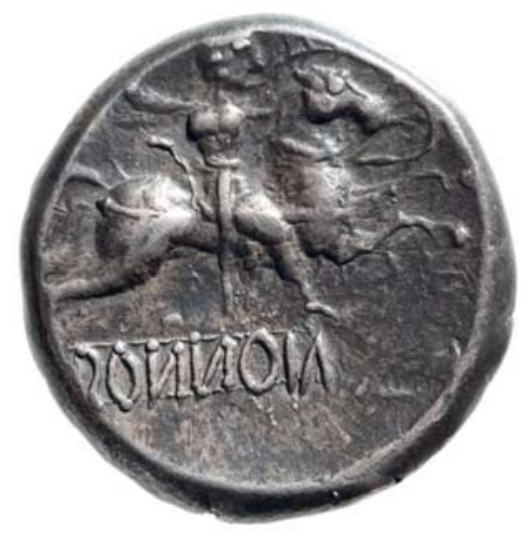

Fig. 14. Tetradrachm with the legend NONNOS, variant Göbl (1994) XIV/2 (1)-13 (cat. no. 151; magnified). - Obr. 14. Tetradrachma s nápisem NONNOS, varianta Göbl (1994) XIV/2 (1)-13 (kat. č. 151; zvětšeno).

II/4-3 and II/4-7) and DEVIL (Göbl 1994, var. IX/1-16 and IX/1-20). The reverse depicts a rider on a horse to the right, holding a sword in his right hand and a rein in his left hand; in the exergue below, there is the legend NONNOS between two lines and the whole is framed with a smooth line with arches turned inwards. Also, this coin is - with the exception of only a few details - iconographically identical to the tetradrachms of the variant Göbl (1994) XIV/1A-8, XIV/1B-9, XIV/1B10 , and XIV/1C-10. Röttger's unpublished catalogue (Röttger 2019) lists 36 pieces of the NONNOS inscribed tetradrachms in question (Tab. 6), 33 of which are preserved or at least depicted. In the largest tetradrachm hoard of Bratislava 1942, the variant was represented by 10 pieces (Ondrouch 1958, nos. 184-193). The weight range of the coins from the Deutsch Jahrndorf hoard is 17.23-16.98 g.

The Deutsch Jahrndorf hoard contained 14 specimens (cat. nos. 140-153; Fig. 4h: 140-141, 4i: 142-153) of the NONNOS inscribed tetradrachms of the variant Göbl (1994) XIV/2 (1)-13. ${ }^{62}$ The variant in question represents a purely Celtic iconography. The obverse bears a male head with short hair to the right and framed with a 'spruce wreath'. The head type is loosely associated with Roman Late Republican issues. An identical portrait can be found on 1/3-tetradrachms of the variant Göbl (1994) XIV/2 (2)-30 and also the anonymous tetradrachms of the variant Göbl (1994) XVI/1-17 are very similar. The reverse differs strongly from the other tetradrachms with the NONNOS legend. Its bears the depiction of a rider on a horse to the right, holding a sword in his right hand and an oval shield in his left hand, suggested by an oval line around the horse's head; this detail is often poorly visible. A characteristic feature of the reverse is the mirror-flipped legend NONNOS in the form २ОИИОИ. А mirror-flipped legend can also be found in the already mentioned 1/3-tetra-

${ }^{62} \mathrm{Ch}$. Röttger distinguished two subvariants of this variant - A and $\mathrm{B}$ - the former of which is attested by a single coin (Röttger 2019, Database no. 36-1 / 1932). Based on the available photograph we cannot exclude that in both cases we are dealing with the same die. drachms (Paulsen 1933, Taf. 35: 830; Göbl 1994, var. XIV/2 (2)) which moreover bears an identical portrait and was probably minted in the same period. The rider depiction features an interesting detail - the belt on which a scabbard is suspended. Also, the rein's attachment to the bridle, the harness straps, and possibly also the saddle are clearly depicted (Fig. 14). This depiction is an important iconographic source providing information on the form and use of shields, the way swords were carried on the belt, the use of swords in mounted combat, and also horse harnesses. The issues of this variant make it possible to observe the gradual wear of the dies, which is also clearly visible on the pieces from the Deutsch Jahrndorf hoard (cat. nos. 141-142, 144150, 153; Fig. 15: 1-5). Röttger's unpublished catalogue (Röttger 2019) lists 201 specimens of the variant in question of the tetradrachms with the NONNOS legend (Tab. 6), out of which 176 are preserved or at least depicted. The largest tetradrachm hoard Bratislava 1942 contained 25 specimens of this variant (Ondrouch 1958, nos. 194-215, 265-267). The weight range of the coins from the Deutsch Jahrndorf hoard is $17.22-16.79 \mathrm{~g}$.

The last tetradrachm type in the Deutsch Jahrndorf hoard is represented by two specimens (cat. nos. 155156; Fig. 4i: 155-156) of the issue with the legend TITTO of the variant Göbl (1994) XV/1-25. The iconography of this coin is purely Celtic, though the harpy motif is derived from Mediterranean mythology. The obverse bears the depiction of a bust of a male (?) youth to the left, before him a branch terminating in pellets (mistletoe?), on the right, there is a wavy twisted line and the whole is framed with a border of prominent dots. On the reverse there is a depiction of a standing Harpy to the right looking to the left, on the right a vertical legend between two lines AINO, continuing below between two lines XIR; a border of marked dots. The iconography of the tetradrachms with the legend TITTO is basically identical to the issues of AINORIX (Göbl 1994, var. I/1-26; cat. no. 31) and FARIARIX or FAPIARIX $^{63}$ (Göbl 1994, var. XI/1-27) - all three were obviously minted in approximately the same period. Röttger's unpublished catalogue (Röttger 2019) lists 28 specimens of the tetradrachms with the TITTO legend (Tab. 6), out of which 26 are preserved or at least depicted. In the largest tetradrachm hoard from Bratislava 1942 , this issue was represented by three pieces (Ondrouch 1958, nos. 249-251). The weight range of the specimens from the Deutsch Jahrndorf treasure is 17.08-17.06 g.

The above exposed overview makes clear the extraordinary richness of the tetradrachm component of the Deutsch Jahrndorf hoard. The treasure included coins with the names of AINIORIX, BIATEC, BVSSVMARVS, BVSV, COBROVOMARVS, COISA, COVIOMARVS, DEVIL (or DEVII), EVOIVRIX (or FAPIARIX), LATVMARVS (IANTVMARVS), NONNOS, and TITTO. The only non represented names are COVNOS (Göbl 1994, var. VIII/ 1-15), FARIARIX or FAPIARIX (Göbl 1994, var. XI/1-27), LATTV (Militký 2013b, 162, nos. 21-22, Fig. 1c: 21-22; 2014), MACCIVS (Göbl 1994, var. XIII/1-22), and the

\footnotetext{
${ }^{63}$ Ch. Röttger $(2013,37)$ proposed the reading FAPIARIX.
} 
Fig. 15. Gradual wear of the dies of the variant Göbl (1994) XIV/2 (1)-13: 1 - cat. no. 151; 2 - cat. no. 141; $\mathbf{3}$ - cat. no. 145; 4 cat. no. 147; 5 - cat. no. 153. - Obr. 15. Príklady postupného opotřebování razidel varianty Göbl (1994) XIV/2 (1)-13: 1 - kat. c. 151; 2 - kat. č. 141 3 - kat. č. 145; 4 kat. c.. 147; 5 - kat. c. 153.

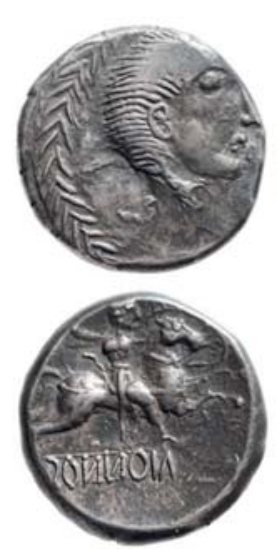

1 (151)

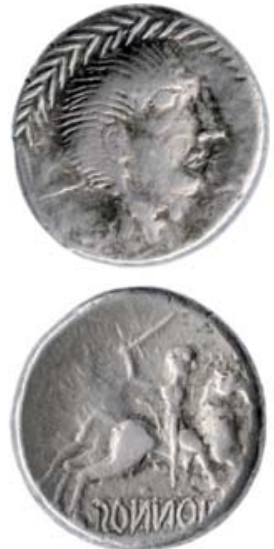

2 (141)
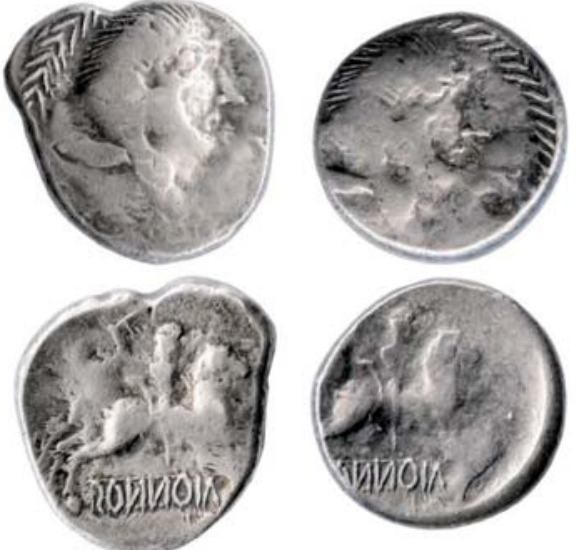

3 (145)

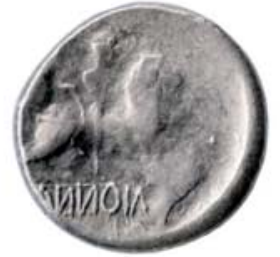

4 (147)
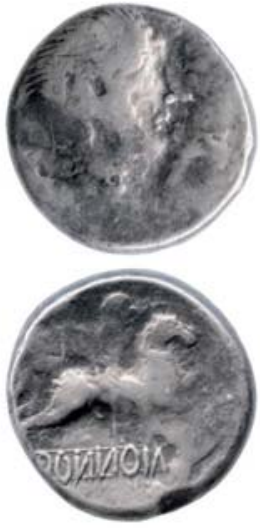

$5(153)$

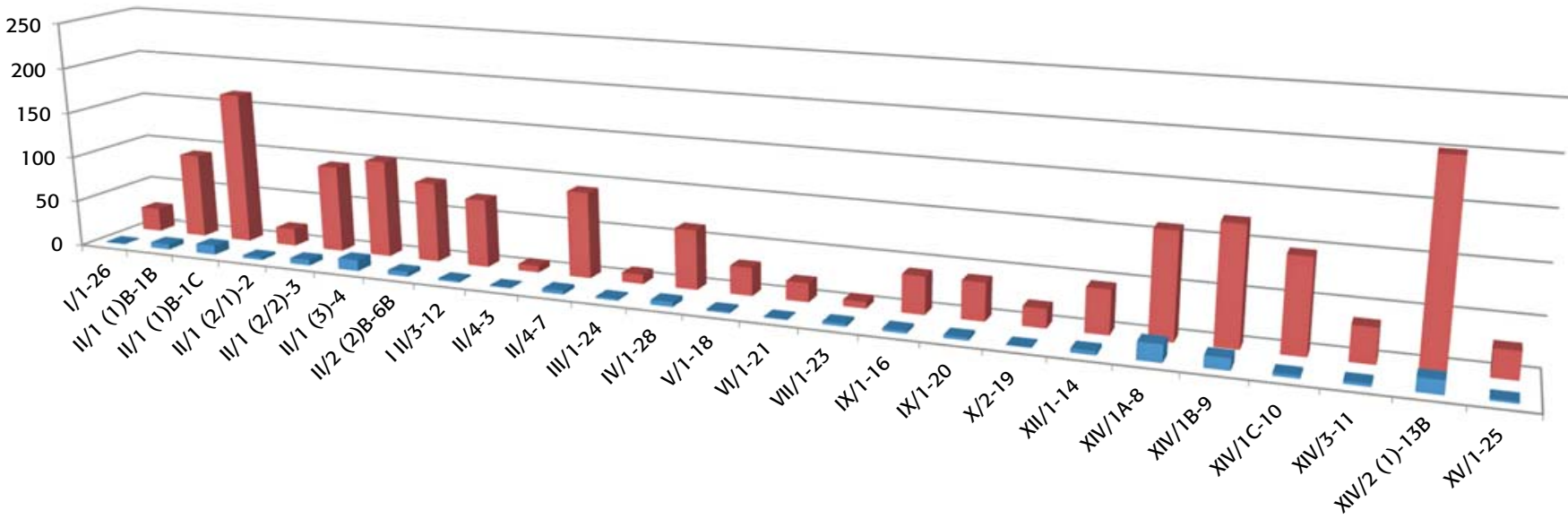

Fig. 16. Frequency of the die combinations (Göbl 1994; Röttger 2019) in the Deutsch Jahrndorf hoard (blue) and their comparison with the overall number of attested specimens (red). - Obr. 16. Grafické znázornění frekvence výskytu kombinací razidel (Göbl 1994; Röttger 2019) v depotu Deutsch Jahrndorf (modře) a jejich početní porovnánís celkovým počtem doložených exemplářu (červeně).

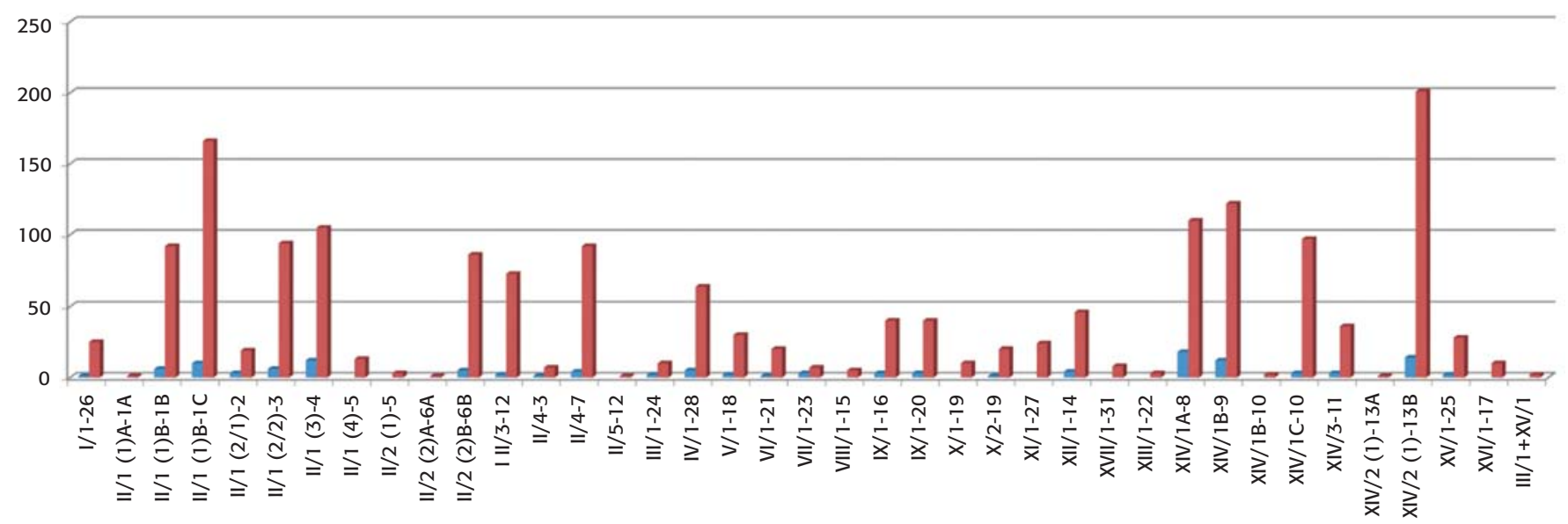

Fig. 17. Frequency of the die combinations (Göbl 1994; Röttger 2019) in the Deutsch Jahrndorf hoard (blue) and their comparison with the overall number of attested specimens (red), including the combinations that are not represented in the hoard. - Obr. 17. Grafické znázornění frekvence výskytu kombinací razidel (Göbl 1994; Röttger 2019) v depotu Deutsch Jahrndorf (modře) a jejich početní porovnání s celkovým počtem doložených exemplárù (červeně), včetně v depotu neodložených kombinací.

anonymous type (Göbl 1994, var. XVI/1-17). Also missing are the rare variants (Tab. 5) with the legends BIATEC, EVOIVRIX (or FAPIARIX), and the hybrids BVSV-
MARVS and TITTO (Tab. 6). The die combinations are represented almost in their entirety (Tab. 6; Fig. 16-17). Significantly, the numerical representation of the tetra- 


\begin{tabular}{|c|c|c|c|c|}
\hline Cat. nos. & Name & Göbl 1994; Röttger 2019 & DJ & $\Sigma$ \\
\hline \multirow[t]{2}{*}{30} & AINORIX & $1 / 1-26$ & 1 & 25 \\
\hline & BIATEC & II/1 (1)A-1A & & 1 \\
\hline $31-36$ & BIATEC & II/1 (1)B-1B & 6 & 92 \\
\hline $37-46$ & BIATEC & II/1 (1)B-1C & 10 & 166 \\
\hline $47-49$ & BIATEC & $11 / 1(2 / 1)-2$ & 3 & 19 \\
\hline $50-55$ & BIATEC & $11 / 1(2 / 2)-3$ & 6 & 94 \\
\hline \multirow[t]{4}{*}{$56-67$} & BIATEC & $11 / 1(3)-4$ & 12 & 105 \\
\hline & BIATEC & II/1 (4)-5 & & 13 \\
\hline & BIATEC & $11 / 2(1)-5$ & & 3 \\
\hline & BIATEC & II/2 (2)A-6A & & 1 \\
\hline $68-72$ & BIATEC & $\mathrm{II} / 2(2) \mathrm{B}-6 \mathrm{~B}$ & 5 & 86 \\
\hline $73-74$ & BIATEC & $11 / 3-12$ & $\mathbf{2}$ & 73 \\
\hline 75 & BIATEC & II/4-3 & 1 & 7 \\
\hline \multirow[t]{2}{*}{$76-79$} & BIATEC & III/4-7 & 4 & 92 \\
\hline & BIATEC & $11 / 5-12$ & & 1 \\
\hline $80-81$ & BVSSVMARVS & $111 / 1-24$ & $\mathbf{2}$ & 10 \\
\hline $82-86$ & BVSV & IV/1-28 & 5 & 64 \\
\hline $87-88$ & COBROVOMARVS & V/1-18 & 2 & 30 \\
\hline 89 & COISA & VI/1-21 & 1 & 20 \\
\hline \multirow[t]{2}{*}{$90-92$} & COVIOMARVS & VII/1-23 & 3 & 7 \\
\hline & COVNOS & VIII/1-15 & & 5 \\
\hline 93-95 & DEVIL (or DEVII) & IX/1-16 & 3 & 40 \\
\hline \multirow[t]{2}{*}{$96-98$} & DEVIL (or DEVII) & $I X / 1-20$ & 3 & 40 \\
\hline & EVOIVRIX (or FAPIARIX) & $\mathrm{X} / 1-19$ & & 10 \\
\hline \multirow[t]{2}{*}{99} & EVOIVRIX (or FAPIARIX) & $\mathrm{X} / 2-19$ & 1 & 20 \\
\hline & FARIARIX (or FAPIARIX) & $\mathrm{XI} / 1-27$ & & 24 \\
\hline \multirow[t]{3}{*}{$100-103$} & LATVMARVS (IANTVMARVS) & XII/1-14 & 4 & 46 \\
\hline & LATTV & $\mathrm{XVII} / 1-31$ & & 8 \\
\hline & MACCIVS & XIII/1-22 & & 3 \\
\hline $104-121$ & NONNOS & XIV/1A-8 & 18 & 110 \\
\hline \multirow[t]{2}{*}{$122-133$} & NONNOS & XIV/1B-9 & 12 & 122 \\
\hline & NONNOS & XIV/1B-10 & & 2 \\
\hline 134-136 & NONNOS & XIV/1C-10 & 3 & 97 \\
\hline 137-139 & NONNOS & $\mathrm{XIV} / 3-11$ & 3 & 36 \\
\hline 140-153 & NONNOS (2ОИИОИ) & XIV/2 (1)-13 & 14 & 201 \\
\hline \multirow[t]{3}{*}{$155-156$} & TIITO & $X V / 1-25$ & 2 & 28 \\
\hline & Anonymous & $\mathrm{XVI} / 1-17$ & & 10 \\
\hline & BVSVMARVS+TITTO & $\mathrm{III} / 1+\mathrm{XV} / 1$ & & 2 \\
\hline
\end{tabular}

Tab. 6. Die combinations of Biatec group tetradrachmas attested so far (Göb) 1994; Röttger 2019), the variants represented in the Deutsch Jahrndorf treasure (DJ) and the overall number $(\Sigma)$ of the documented specimens of the single variants (after Röttger 2019). - Tab. 6. Doposud známé kombinace razidel tetradrachem skupiny Biatec (Göbl 1994; Röttger 2019), varianty zastoupené $v$ depotu Deutsch Jahrndorf $(D J)$ a celkový počet $(\Sigma)$ doložených exemplárư v rámci jednotlivých variant (podle Röttger 2019).

drachms variants in the studied treasure corresponds with the numbers of coins attested for the individual die combinations. The Deutsch Jahrndorf hoard thus turns out to be - along with the Bratislava 1942 treasure (Ondrouch 1958) - the most significant find assemblage of the Biatec group tetradrachms.

\section{The period value, the concealment date, and the significance of the hoard}

At the moment of its concealment, the Deutsch Jahrndorf hoard - or more specifically its preserved part - corresponded to almost 250 tetradrachms (using the Roman ratio of gold/silver value of $1: 12.5$ in which case one stater corresponds roughly to five tetradrachms). ${ }^{64}$ This is the second largest deposit of coins minted in Celtic Bratislava, the largest one still being the treasure discovered in Bratislava in 1942 which contained 270 tetradrachms (Ondrouch 1958). These two assemblages are followed by much smaller hoards from Bratislava itself (Paulsen 1933, 103-105; Ondrouch 1964, 44-46, no. 61-62, 63a-66) or from Reca (Paulsen 1933, 107-109; Ondrouch 1964, 42, no. 57, 52, no. 91 and 68, no. 133).

We know that the Deutsch Jahrndorf hoard was concealed in a pottery vessel (which has not come down to us), as was also the case with some other hoards of the Biatec group coins - e.g. Bratislava 1937 (Ondrouch 1964, 46, no. 65), Reca (Anonym 1905, 3317; Paulsen 1933, 107-109; Ondrouch 1964, 42, no. 57, 52, no. 91 and 68, no. 133), Wien-Simmering (Kenner 1880; 1895; Paulsen 1933, 117-119; Ruske 2011a, 71, no. V27), Schottwien (Ruske 2011a, 69, no. V23; 2011b), and probably also Gerlitzen (Paulsen 1933, 123-124; Ruske 2011a, 63, no. V6) and Schwechat (Paulsen 1933, 117; Ruske 2011a, 69, no. V24). Also, the as yet unpublished find from Potzneusiedl in Austria was probably deposited in a vessel. Ceramic vessels thus apparently very often served for the purpose of burying treasures of Bratislava coins.

A key issue concerning the Deutsch Jahrndorf hoard is, in what milieu it was deposited. This question is hard to answer given the incertitude concerning the precise place of discovery. As a result, we cannot be sure whether the hoard was concealed in an inhabited area or in open landscape. No other La Tène period finds are reported from the rather large territory of the Deutsch Jahrndorf village which - moreover - is not sufficiently studied to provide useful information. In any case, the find most probably can be associated with a route heading south from the Bratislava oppidum - the find-spot is just $15 \mathrm{~km}$ distant from the oppidum acropolis. Several other treasures (Wien-Simmering, Schwechat, the surroundings of Salzburg; Fig. 5) and individual finds are known from the area further upstream on the Danube and towards the Alps. These finds are now joined by the newly identified Potzneusiedl treasure located ca. $15 \mathrm{~km}$ west of Deutsch Jahrndorf and $15 \mathrm{~km}$ south of the Bratislava oppidum acropolis. All these finds probably indicated the zone under the influence or direct control of the Bratislava oppidum. In the case of the Alpine finds, the explanation can be found in the relations between Bratislava and Roman Republican Italy. Worthy of attention and further study is the relation between the finds from Vienna (Wien-Simmering and Wien-Schwechat; Fig. 5) with the contemporary flatland settlement in the territory of present-day Vienna in which local coin production is clearly documented by finds of flan moulds (Adler-Wölfl 2012; Adler-Wölfl Mosser 2015). However, the find from Deutsch Jahrndorf - and also that from Potzneusiedl - most probably come from a zone directly controlled by Bratislava.

The money deposited in the Deutsch Jahrndorf treasure was probably - or almost certainly - accumulated directly in the Bratislava oppidum. A key question

\footnotetext{
${ }^{64}$ It is necessary to stress that this is only a hypothetical model.
} 
is, whether it happen over a long or short period of time. Although we naturally do not know the answer to this question, we may consider the long term scenario as more likely given the large typological array of both gold and silver coins.

The questions of the chronology of Bratislava coin production discussed above are tightly linked to the issue of the date of the Deutsch Jahrndorf hoard concealment. Also, in this case we cannot go beyond hypotheses. In view of the presence of most tetradrachm variants attested so far (Tab. 6) it seems probable that the treasure was deposited in the ground in a time close to the oppidum's decline or even abandonment. An important sign is the absence of the tetradrachms with the legend LATTV which was probably the very latest of the inscribed issues (Militky 2013b, 173); the exact dating of this type is open to discussion as suggested above. However, the absence of some types need not necessarily be only a chronological indicator. Given our present state of knowledge on the Bratislava coinage chronology, the concealment of the Deutsch Jahrndorf hoard may date to the third quarter of the $1^{\text {st }}$ century $\mathrm{BC}$; a more precise date cannot be determined.

Another open and to some extent unanswerable question is the identity of the issuers of the Deutsch Jahrndorf coins, i.e. of the Bratislava oppidum elites. Traditionally, they are associated with Boii whose seats are assumed to have been in Bohemia (Militký 2015a, 169). The Boian element is represented in Bratislava by the gold denominations and by the silver obols of the Karlstein type; on the contrary the silver tetradrachms of the Biatec group and the hemidrachms of the Simmering type are specifically local denominations without any parallel in the Celtic coinages. The obvious dominance of large silver coins clearly shows that we find ourselves in a frontier area between the zone of bimetallic coinages characteristic of the Boii area and of the monometallic silver zone of the east Celtic world (Militký - Karwowski $2013,26)$. Coins by themselves cannot naturally answer the questions of tribal identity; in the case of Bratislava, we can however clearly prove the close association of this site with the Bohemian milieu and thus with the presumed Boii (Militký 2015b, 93-94).

The Deutsch Jahrndorf hoard represents a unique source for the study of the Bratislava coin production. Although for many decades, its full significance was not apparent, the new study of the assemblage significantly solidified our knowledge of the Bratislava Celtic coinage. The new finds of Roman style architecture in the Bratislava oppidum acropolis make us better understand the contacts between the local elites and the Roman Republic. The study of the hoard in question contributes to the same topic from the numismatic point of view.

\section{Acknowledgements}

This study was supported by project Nr. 18-20096S "Mobility of materials and life cycles of artefacts: archaeometry of metals and glass of the La Tène and Early Roman period" of the Czech Science Foundation (GA ČR).

English by Jan Kysela

\section{Souhrn}

\section{1. Úvod}

Detailní zpracování starých nálezových souborů keltských mincí je dnes jedním $z$ důležitých úkolů numismatického bádání. Je však paradoxem, že revizi většiny starých pokladů obsahujících mince skupiny Biatec se v posledních desetiletích nikdo nevěnoval. Do této kategorie spadá také zde prezentovaný poklad odkrytý u obce Deutsch Jahrndorf v rakouském Burgenlandu, který je zároveň jedním $\mathrm{z}$ nejdůležitějších pramenů $\mathrm{k}$ bratislavskému keltskému mincovnictví.

\section{Místo nálezu, nálezové okolnosti}

\section{a přehled dosavadního zpracování depotu}

Depot byl objeven u obce Deutsch Jahrndorf (okres Neusiedl am See, Burgenland), jejíž katastrální území dnes leží na hranici se Slovenskem a Madarskem (obr. 1). V květnu 1855 jej při kopání hraničního příkopu mezi arcivévodským a obecním pozemkem (Humer 2006, 26) našel chalupník Paul Eder. Místo odkrytí depotu leží někde v okolí dvora Karlhof (Schmeller 1965, 225), pravděpodobně v linii severovýchodní hranice arcivévodských pozemků (obr. 2).

Depot byl uložen v keramické nádobě, která se nedochovala. Co se dělo s mincemi bezprostředně po nálezu nevíme, ale je jisté, že byla snaha je zpeněžit. Podstatná část nálezu se dostala do rukou místního učitele, který je v Bratislavě nabídl k prodeji nejmenovanému zlatníkovi (Seidl 1855, 302). Mince byly určeny k roztavení, před kterým je zachránil prof. Karl Julius Schröer (1825-1900). Díky němu se podařilo zachovat 26 zlatých a 101 stříbrných ražeb (Kupido 1866, 98), které jsou dnes uloženy v Kunsthistorisches Museum ve Vídni (41 ks, zkratka KHM Wien) a v Magyar Nemzeti Múzeum v Budapešti ( 86 ks, zkratka MNM Budapest). Je však zcela jisté, že depot původně obsahoval více mincí, jak dokládá 163 dochovaných či doložených ražeb (tab. 1).

Pokladem z Deutsch Jahrndorfu se opakovaně zabývala celá řada autorů, doposud však nebyl jako celek zveřejněn a také většina tetradrachem $z$ MNM v Budapešti zůstala nepublikována. V tomto příspěvku je značná pozornost věnována právě kritickému rozboru identifikovatelného obsahu nálezu. Zde je třeba zvláště zdůraznit význam nepublikované databáze Ch. Röttgera (Röttger 2019), která byla autorům k dispozici a s jejíž pomocí se také podařilo doložit, že dvě mince tradičně spojované s pokladem (obr. 3), do něho ve skutečnosti nepatří.

\section{Katalog mincí z depotu}

\section{Současný stav poznání mincovnictví na bratislavském oppidu}

\subsection{Základní charakteristika}

Problematice bratislavských mincí skupiny Biatec, typu Simmering a do jisté míry i zlatým nominálům, byla dlouhodobě věnována značná pozornost (Kupido 1866; Kenner 1880; 1895; Eisner 1925). Souhrnně se těmito ražbami zabýval $R$. Paulsen (1933, 74-127, Taf. 28-43) a posléze $V$. Ondrouch (1958). Zlatým ražbám se věnoval také K. Castelin (1965, 15-16), který je v rámci jím definovaného relativně-chronologického systému začlenil do období ražby D. Ke shrnutí poznatků se znovu vrátila E. Kolníková (1991). Autorem základní typologické práce stříbrných ražeb je $R$. Göbl (1994). Jeho typologie je velice precizní, chronologické schéma však pouze hypotetické. V posledních letech se bratislavské mincovnictví stalo opět předmětem intenzivního zájmu několika badatelů. Jedním z impulsů byly také nové objevy římské, resp. římsko-keltské architektury na akropoli bratislavského oppida, tedy na dnešním hradním návrší, odkud pocházejí také nové mincovní nálezy $(\mathrm{Mu}$ silová-Barta-Herucová /eds./ 2014; Musilová-Kolníková-Hložek 2015).

Dosavadní poznatky shrnula v posledních letech opakovaně E. Kolníková (Kolníková 2012a; Kolníková-Bakos - Pauditš 2018, 157-159). Velice důležité je zveřejnění nového mincovního nálezu z rrímsko-keltské stavby I na akropoli oppida (Musilová-Kolníková - Hložek 2015). Přehledy problematiky zveřejnil J. Militký v souvislosti s publikací dvou depotů bez lokality (Militký 2013a; 2013b), 
v rámci nálezového fondu mincí typu Simmering z oppida Stradonice (Militký 2015a, 119-123), v rámci shrnutí keltského mincovnictví v severovýchodním Rakousku (Militký 2020, 313-318) a také jako přehlednou stat ve vztahu $\mathrm{k}$ bójskému prostředí (Militký $2015 b$, 89-91). V souvislosti se zveřejněním statéru s kontramarkovaným (?) nápisem DEVIL se J. Fröhlich pokusil o interpretaci jmen na stříbrných ražbách (Fröhlich 2011). Nové čtení nápisů některých tetradrachem navrhl Ch. Röttger (2013). Tento autor se rovněž pokusil o sestavení nové relativní a především absolutní chronologie (Röttger 2015), jeho schéma je však do značné míry v rozporu s dosavadními dlouhodobými poznatky o chronologii pozdní doby laténské. Obraz distribuce bratislavských mincí doplňuje také několik nově zveřejněných nálezů z území Slovenska (Čambal Budaj 2009; Budaj - Čambal 2013a; 2013b; 2016) nebo revize starého nálezu (Schottwien: Ruske 2011b; Vrtel - Maretta 2018; Röttger 2020).

Mince skupiny Biatec byly dlouhodobě považovány za tetradrachmy. Avšak R. Göbl (1994) je ve své terminologii označil za hexadrachmy, což bylo akceptováno v řadě dalších prací (Kostial 2003, č. 61-87; Militký 2004; 2013a; 2013b; 2015a). Nyní se ovšem ukazuje, že jde skutečně o tetradrachmy s tradičním attickým standardem $(17,26 \mathrm{~g})$. Hmotnostní standard drachem (v Bratislavě ale neražených), by tak odpovídal hodnotě $4,315 \mathrm{~g}$ a půldrachem (hemidrachem) pak 2,15 g, což ideálně koresponduje s hmotnostm mincí typu Simmering (Paulsen 1933, Taf. 36-42, 43: 971-978, Göbl 1994, Taf. 8: 23-33). Název těchto ražeb je odvozen podle jejich zastoupení v depotu Wien-Simmering (Kenner 1880; 1895; Paulsen 1933, 117-119; Ruske 2011a, 71, č. V27).

Pro pochopení celého mincovního systému jsou klíčové právě nálezy těchto mincí. Dnes je zcela nezpochybnitelné, že centrem jejich produkce bylo oppidum na území dnešní Bratislavy (např Pieta 2008, 111; Kolníková 2012a, 206-213). Kromě řady jednotlivě objevených mincí odtud známe již nejméně osm (snad i devět) hromadných nálezů a depotů, ve kterých byly mince skupiny Biatec zastoupeny (Ondrouch 1964, 44-46, č. 61-66; Kolníková 1991, 23 27; Militkú 2004; Kolníková 2012a, 215; obr. 5). V roce 2009 se na hradním návrší, původní akropoli oppida, podařilo prokázat existenci architektury zděné po římském způsobu (Šedivý - Štefanovi čová /eds./ 2012, 197-205; Musilová - Barta - Herucová /eds./ 2014; Musilová - Kolníková - Hložek 2015)

Z interiéru stavby I pochází zatím poslední depot mincí, který kromě stříbrných mincí skupiny Biatec a typu Simmering obsahoval především zlaté statéry (Musilová - Kolníková - Hložek 2015, 253-256, obr. 13). Mince byly původně považovány za možnou stavební obětinu, byly však nalezeny ve vrstvě 7 nad podlahou, tedy ve stratigraficky nejstarší vrstvě již vyplňující tuto část interiéru stavby I (Musilová - Kolníková - Hložek 2015, 247-251, obr. 7-10). Ražby se tak do nálezové pozice dostaly za nejasných okolností až $\mathrm{v}$ době, kdy již stavba zřejmě zanikala - podle jejich rozmístění ve vrstvě můžeme uvažovat o sekundární pozici, mohly být např. původně zazděny ve vyšší partii stavby. V každém případě však tento nález s vysokou pravděpodobností dokazuje, že římské stavby pocházejí z období, kdy byly na lokalitě raženy mince skupiny Biatec a typu Simmering.

Při snaze o charakteristiku bratislavského mincovnictví je třeba zdůraznit, že i na této lokalitě existoval peněžní systém tvořený zlatými a stř́ibrnými nominály. Existence mincovních systémů založených na zlatě a stříbru je zřejmá již od období okolo poloviny 3. století před Kristem v oblasti koridoru Jantarové stezky a v Čechách (Militký 2015b, 71-77; 2019). Zřetelné bimetalické peněžn systémy fungovaly také v průběhu oppidálního období v Čechách i na Moravě (Militký 2015a; 2015b, 79-87). Je tedy evidentní, že peněžní systém stojící na zlatě a stříbru fungoval i v Bratislavě, což výtečně dokládají i mincovní nálezy. Lze tedy odmítnout úvahy E. Kolníkové o ražbě zlatých beznápisových i nápisových statérů v období před ražbou stříbrných tetradrachem (Kolníková $2012 a$ 206; Musilová - Kolníková - Hložek 2015, 273-274). Tato hypotéza je zcela v rozporu se svědectvím depotu z Deutsch Jahrndorf i pokladu $\mathrm{z}$ akropole bratislavského oppida (Musilová - Kolníková - Hlo žek 2015, 253-256, obr. 13). Naprosto nereálná je také představa E. Kolníkové o norickém původu drobných mincí typu Karlstein a jejich datování pouze do závěrečného horizontu bratislavského oppida (naposledy Kolníková - Bakos - Pauditš 2018, 159). V kon- textu pohledu na typologii drobné mince oppidálního období v Čechách, na Moravě, v naddunajském Rakousku či Trenčinských Bohuslavicích (souhrnně např. Militký 2015a,84-88) je typ Karlstein s jistotou ražen po celou mladší fázi oppidálního období (LT D1b) a tedy určitě i po celou dobu trvání oppida v Bratislavě. Jde prostě o součást jednoho měnového bimetalického systému, tvořeného z části tradičními tzv. bójskými nominály (zlaté mince a typ Karlstein) a specifickými lokálními stříbrnými nominály (tetradrachmy skupiny Biatec, 1 1/2 drachmy skupiny Biatec a hemidrachmy typu Simmerig). Samostatnou otázku pak přestavuje diskuze o Bójích a Bratislavě, kterou ovšem numismatika sama o sobě nemůže vyřešit.

\subsection{Nominály mincovního systému na bratislavském oppidu}

Mincovní systém produkovaný na bratislavském oppidu sestává z několika zlatých a stříbrných nominálů, které v rámci relativně-chronologického systému K. Castelina (Castelin 1965, 15-16) patří do úseku ražby D. Zlaté nominály přímo navazující na produkci zlatých mincí v Čechách reprezentují mušlové beznápisové statéry a také mince se jménem BIATEC ( $t a b$. 4: 1-18; obr. $7: 1-17$ ), a to včetně jejich jen omezeně produkovaných dílů - $1 / 3$ statérů a $1 / 8$ statérů bez nápisů i se zkratkami jména BIAT. V souvislosti se zlatými nápisovými ražbami je nutné připomenout také čtyři statéry se jménem NONNOS pocházející z depotu na akropoli bratislavského oppida (Musilová - Kolníková - Hložek 2015, 255, č. 10-13, obr. 13: 10-13; obr. 7: 19-22). Je důležité, že se v tomto nálezu vyskytly společně i tetradrachmy a hemidrachma typu Simmering. Jde o další důležité potvrzení současného užívání všech těchto nominálů v rámci jednoho peněžního systému.

Jaký byl penežní vztah mezi zlatými statéry a stř́ibrnými tetradrachmami je těžké odhadnut. Velmi pravděpodobně však reflektoval římský poměr zlata a stř́bra, který se za Augusta ustálil na $1: 12,5$. V takovém př́ipadě by jednomu statéru odpovídalo přibližně pět tetradrachem a 40 půldrachem typu Simmering.

Nápisy BIATEC a NONNOS na zlatých nominálech mají obrovský interpretační význam, nebot umožňují jednoznačné propojení se stř́brnými ražbami skupiny Biatec a typu Simmering. Prostřednictvím pokladi̊ z Deutsch Jahrndorfu (1855), Bratislavy (2009) a dalších jednotlivých ztrátových exemplářu známe rovněž doklady o intenzivní paralelní produkci beznápisových mušlových statérů $\mathrm{v}$ řadě typů a také jejich dílů. Úlohu těchto ražeb osvětluje především poklad $z$ Deutsch Jahrndorfu a proto je těmto mincím v následném textu věnována obsáhlá pozornost. Beznápisové statéry jsou úzce provázány se závěrečným horizontem na oppidu Stradonice (Militký 2015a, 49).

Nejvýraznějším typem mincí ražených v Bratislavě jsou bezesporu stříbrné tetradrachmy skupiny Biatec. Jde o zcela jedinečné nominály s attickým mincovním standardem, které také $\mathrm{z}$ ikonografického a epigrafického hlediska nemají ve středoevropském keltském mincovnictví obdoby. Nejnápadnější stránkou těchto mincí jsou latinkou psaná osobní jména umístěná zpravidla na rubní straně - AINORIX, BIATEC, BVSV, BVSSVMARVS, COBROVOMARVS, COISA, COVIOMARVS, COVNOS, DEVIL (nebo DEVII), EVOIVRIX (nebo FAPIARIX), FARIARIX (nebo FAPIARIX), LATVMARVS (IANTVMARVS), MACCIVS, NONNOS, TITTO (Göbl 1994, Taf. 1) a nově i LATTV (Militký 2013b, 162, č. 21-22, obr. 1c: 21-22; 2014). Kromě toho známe také jednu anonymní tetradrachmu bez nápisu (Göbl 1994, var. XVI/1-17). Přítomnost nápisů psaných latinským písmem je jedinečným důkazem výrazného ovlivnění elit produkujících mince v Bratislavě římským republikánským prostředím. Tato skutečnost získala v kontextu nových nálezů římské architektury na akropoli bratislavského oppida zcela novou dimenzi. S analogickým jevem se v rámci keltského mincovnictví setkáváme v Noriku, jehož centrem bylo oppidum Magdalensberg s lokální mincovní produkcí (Krmnicek 2010) i s existencí římských zděných staveb v kontextu pozdní doby laténské. Je pravděpodobné, že především na této lokalitě bylo raženo více typů stř́ibrných tetradrachem s latinkou psanými nápisy (Göbl 1973, 83-97). Magdalensberg byl již v době před římskou okupací Norika v roce 15 před Kristem, kdy se Norikum stalo klientským královstvím Římanů, pod silným římským vlivem. Chronologie norického mincovnictví je dnes značně komplikované téma, ale určitě existovalo i v době existence bratislavského oppida. Je tedy zřejmé, že použití 
písma na mincích je jedním z významných projevů římské kulturní infiltrace do keltského prostředí na středním Dunaji.

Produkce zvláštních nominálů odpovídajících třem hemidrachmám typu Simmering (1 $1 / 2$ drachma) se jmény BIATEC a NONNOS byla minoritní. Tyto mince známe pouze jako jednotlivé exempláře (Paulsen 1933, Taf. 35: 829-830; Göbl 1994, var. II/6-29 a XIV/2 /2/-30; Kolníková 1995), zatím zcela chybí v hromadných nálezech a jejich úloze v rámci peněžního systému nerozumíme. Důležitý je ovšem fakt, že typ s nápisem BIATEC (Göbl 1994, var II/6-29) napodobuje římské republikánské denáry typu $R R C$ č. 407/1 nebo 407/2 a 464/1 ražené v letech 68 a 46 před Kristem. Jde tedy o nejmladší post quem datovanou bratislavskou ražbu musela totiž vzniknout nejdříve v roce 46 před Kristem (tab. 2).

Velice intenzivní byla produkce hemidrachem typu Simmering. Jde o ražby s hmotností v rozmezí 2 až 2,4 g, ražené ve více variantách mnoha razidly. Na líci jsou různé variace vavřínového věnce nebo motivu v podobě Y lemovaného kroužky. Na rubu se vždy vyskytuje motiv koně ve skoku doleva a s různými kombinacemi kuličkovitých závěsků pod břichem. Nejdůležitějším rozlišovacím prvkem jsou ovšem symboly nad koníkem - pták, prázdný kroužek, kroužek s křižem nebo kuličkou, roseta tvořená kuličkami, kulička s jemnými krátkými paprsky a půlměsíc (Paulsen 1933, Taf. C: 1-21). Typologie těchto mincí byla v literatuře dosud zpracována jen částečně (Kolníková 1991, 82, obr. 17-24; Göbl 1994, Taf. 8: 23-33). Zcela novou typologii až do úrovně kombinací razidel vytvořil v rámci své databáze Ch. Röttger (2019) - zatím však nebyla publikována.

Součást bratislavského měnového systému tvoří také oboly typu Karlstein, které představovaly nejdrobnější nominál v rámci celého měnového systému. Jde o mince s hladkým lícem bez obrazu a motivem koně na rubu (nejčastěji orientovaného doleva). Oboly typu Karlstein známe $\mathrm{z}$ řady jednotlivých nálezů v areálu oppida (Kolní ková-Kovár 2010, 70, obr. 1: 5-10; Musilová-Kolníková-Hložek 2015, 276, tab. 5) a hromadný nález, resp. obsah měšce s ražbam tohoto typu pochází $\mathrm{z}$ archeologického výzkumu na hradním ná dvoří (Vrtel 2011, 268, obr. 6-7; Lesák 2016, 120). Tento typ byl dlouhou dobu považován za západonorický (Göbl 1973, Taf. 44: N; Kellner 1990, 181). Dnes je zřejmé, že jde o typickou ražbu pro bójskou mincovní oblast. I v př́ipadě oppida Bratislava reprezentují oboly typu Karlstein, společně se zlatými nominály, jasně identifikovatelnou bójskou složku tamního mincovního systému. Lze je spojit s nejmladší fází bójského mincovnictví (LT D1b-D2) a datovat do průběhu 1. a především 2. třetiny 1 . století před Kristem (např Militký 2013a, 108; 2015a,84).

\subsection{Chronologie mincovního systému bratislavského oppida}

Složitou problematiku představuje chronologie bratislavských mincí. Ta vychází $z$ archeologického kontextu, datací post quem některých variant tetradrachem skupiny Biatec napodobujících římské republikánské předlohy ( $t a b$. 2) a také $z$ relativně-chronologických vztahů mezi jednotlivými emisemi. Je paradoxem, že ačkoliv jde o jedny $z$ nejlépe datovatelných keltských mincí ve střední Evropě, není pohled na jejich datování vůbec jednotný.

Doposud nejkomplexnější pohled přinesla práce R. Göbla (Göbl 1994), který v rámci typologické analýzy tetradrachem skupiny Biatec vypracoval také velice detailní a přesnou chronologii jednotlivých emisí, které roztřídil do 15 horizontů a pokusil se je datovat prakticky s přesností jednoho roku v rozmezí let 51-41 před Kristem (Göbl 1994, 27-33, Taf. 14). Konec produkce spojoval s úpadkem bratislavského oppida v důsledku porážky ve válce s Dáky v roce 41 před Kristem (Göbl 1994, 37-45). Jeho absolutní datování i náplně jednotlivých horizontů však nelze považovat za reálné jde o čistě hypotetický typologicko-chronologický konstrukt. Nejistá je i jeho předpokládaná relativně-chronologická následnost jednotlivých typů.

V nedávné době zveřejnil nový chronologický koncept bratislavského mincovnictví Ch. Röttger (2015). Podle této práce reprezentuje mincovnictví šest fází, datovaných do rozmezí let přibližně 40-9 před Kristem. Ke každé fázi přiřadil tento autor konkrétní varianty (Röttger 2015, 203-207). Hlavní oporou jeho velmi pozdního chronologického schématu je předpokládaná skutečnost, že předlohami tetradrachem s hlavami pozdně republikánského chara- kteru jsou ražby císaře Augusta (27 př. Kr. - 14 po Kr.). Další ze jmenovaných dokladů údajné pozdní chronologie je výskyt stejného rubního motivu na dvou tetradrachmách s nápisem BIATEC (Göbl 1994, var. II/3-12 a II/5-12) a na britské bronzové minci nápisem VER (Röttger 2015, obr. 7). Tato ražba je datována do let asi 25 před Kristem až 10 po Kristu (Cottam et al. 2010, 131, č. ABC 2676). Shoda je zjevná, avšak spíše lze považovat za pravděpodobné, že předlohou uvedené anglické ražby byly právě bratislavské mince. Datování většiny variant bratislavské mincovní produkce do průběhu vlády císaře Augusta (Röttger 2015, 204-206) lze $z$ řady důvodů důrazně odmítnout.

Takto pozdní chronologie je v rozporu s dlouhodobě precizovanou archeologickou chronologií celé lokality, která bývá zpravidla datována do rozmezí 2 . a 3. čtvrtiny 1 . století před Kristem. Tomuto schématu odpovídá také chronologie importovaných římských amfor (Kysela-Olmer 2014, 184) či skla (Musilová 2012, 369, 371) pocházejících $z$ interiérů římských staveb na akropoli oppida. Tyto kategorie nálezů naznačují datování vzniku římských staveb ještě před 40. léta 1. století před Kristem. Proti datování s dominancí augustovského horizontu stojí také naprostá absence Augustových mincí či eraviských napodobenin republikánských denárů (k mincovnictví např. Torbágyi 1984; Haupt - Nick 1997; Kolníková 2005; Torbágyi - Vida 2020) v areálu bratislavského oppida (Musilová Kolníková - Hložek 2015, 278, tab. 6). V protikladu k bratislavskému oppidu je spektrum mincovních nálezů pocházejících z nedalekého výšinného sídliště Děvín. Na této lokalitě, kde o římské vojenské přítomnosti v Augustově době není pochyb (Harmadyová 2012, 271-272), známe depot eraviských napodobenin republikánských denárů (Plachá - Fiala 1998) a pochází odtud celkem 19 ražeb císaře Augusta (Musilová - Kolníková - Hložek 2015, 278, tab. 6). Navíc lze s jistotou konstatovat, že eraviské napodobeniny římských republikánských denárů, a samozřejmě ani Augustovy mince, neznáme $z$ žádného naddunajského oppida. Zdá se tedy, že jde o dva chronologicky odlišné horizonty: prní reprezentuje mincovní systém zlatých a stříbrných mincí ražených na bratislavském oppidu, druhý reprezentuji eraviské napodobeniny řimských republikánských denárů s dominancí produkce a nálezového výskytu v augustovském horizontu.

Za současného stavu poznání lze tedy počátek ražby tetradrachem i celého měnového systému v Bratislavě nejspíše datovat do průběhu 60. let 1 . století před Kristem. Konec bratislavského mincovnictví spadá, podle chronologie R. Göbla (Göbl 1994, 37-45), na závěr 40 . let 1 . století před Kristem, kdy měli být Bójové a Tauriskové zdecimováni dáckým králem Burebistou (Dobesch 1994; 1995; Pieta 2008, 45, 50). Tato událost měla být i př́ičinou ukončení mincovní činnosti na bratislavském oppidu. Stejný chronologický koncept nastínila i E. Kolníková (1991, 51-53; 2012a, 215). Jestli produkce mincí skupiny Biatec tedy přetrvala do 30 . let 1 . století před Kristem, není jasné. Stále více indicií ovšem naznačuje, že období okolo roku 40 před Kristem zřejmě není okamžikem zániku lokality a nelze tak vyloučit, že ražba (?) nebo alespoň oběh bratislavských mincí souvisí zřejmě i s obdobím 30. let 1. století před Kristem. Tomu by mohl odpovídat i doprovodný nález spony typu Almgren 238 (Almgren 1923) objevený údajně společně s bratislavskými ražbami na Devínské Kobyle u Bratislavy (Budaj - Čambal 2016, 275-277, obr. 4). Oběh tetradrachem skupiny Biatec na přelomu 40. a 30. let 1. století před Kristem dokládá depot Gerlitzen (Paulsen 1933, 123-124; Ruske 2011a, 63, č. V6), kde byl kromě bratislavských a norických tetradrachem zastoupen také rímský republikánský kvinár typu $R R C$ č. 489/3 z roku 43-42 před Kristem. Skutečnost, že mince byla ražena $\mathrm{v}$ příalpských oblastech může odrážet její nedlouhý čas oběhu. Ačkoliv bylo v depotu zastoupeno pouze 19 bratislavských tetradrachem, jde o dosti početné spektrum variant, které byly velmi pravděpodobně raženy před rokem 43-42 před Kristem. Přináší tak další velmi důležitou oporu datování celého mincovního systému. Lze tedy shrnout, že datace bratislavského mincovního systému spadá do 60 . až 40 ., případně i 30. let 1. století před Kristem.

\subsection{Svědectví depotů obsahujících bratislavské mince a jejich kategorizace}

Hromadné nálezy bratislavských mincí lze rozdělit do pěti skupin definovaných podle druhů zastoupených mincí. Prvou reprezentujî 
depoty obsahující společně zlaté a stříbrné nominály (obr. 5: 1-3), druhou skupinu reprezentují depoty obsahující pouze tetradrachmy skupiny Biatec (obr. 5: 4-20), tř̌etí skupinu depotů reprezentují nálezy obsahující tetradrachmy skupiny Biatec a hemidrachmy typu Simmering (obr. 5: 21-28), čtvrtou skupinu depotů reprezentují nálezy obsahující pouze hemidrachmy typu Simmering (obr. 5: 29-31) a pátou skupinu depotů představují soubory obsahující oboly typu Karlstein (obr. 5: 32).

\subsection{Surovinové zdroje bratislavského mincovnictví}

Velmi důležitou otázkou spojenou s bratislavským keltským mincovnictvím je původ kovu používaného k ražbě mincí. Otázce původu zlata pro ražbu keltských mincí v Čechách byla v poslední době věnována dosti obsáhlá pozornost v rámci zpracování nálezů ze stradonického oppida (např. Militký 2015a, 156-158). Pro oblast Slovenska byla k tomuto tématu nedávno zveřejněna obsáhlá studie (Kolníková - Bakos - Pauditš 2018, 144-145). Hlubinná těžba není zatím spolehlivě doložena, pomineme-li určité náznaky možné př́ipovrchové těžby na oppidu Závist (Militký 2015a, 158). Prakticky jisté je však rýžování a následná distribuce zlata, zřejmě i na značné vzdálenosti.

$\mathrm{V}$ případě zlata použivaného $\mathrm{v}$ Bratislavě $\mathrm{k}$ ražbě zlatých statérů a jejich dílů je jeho původ nejasný. Je však jisté, že zlato muselo být do Bratislavy dováženo - jako pravděpodobná forma se jeví zvláště ingoty. $\mathrm{V}$ této souvislosti je nutné připomenout existenci zlatého tyčinkovitého ingotu ze Slovenského Grobu (Budaj- Čambal 2013a, 213, č. 25, obr. 2: 25). Jeho hmostnost 6,57 g prakticky ideálně odpovídá vahám mušlových statérů ražených v Bratislavě, ingot má však výrazně vyšší ryzost 99,1%. Datování ingotu do období ražby v Bratislavě je otevřená, ale nepřímo ji potvrzují take společně nalezené hemidrachmy typu Simmering (Budaj-Čambal 2013a, obr. 2: 2-23).

Ještě daleko složitější problém představuje otázka pưvodu stříbra doby laténské ve střední Evropě. I této otázce byla v poslední době věnována obsáhlá pozornost v souvislosti s nálezy $\mathrm{z}$ oppida Stradonice (např. Militký 2015a, 156-157) a pro oblast Slovenska byla nedávno publikována obsáhlá studie (Kolníková Bakos - Pauditš 2018, 145-146). Na rozdíl od zlata je získávání stř́ibra možné pouze hlubinným hornickým způsobem. Pro české země jednoznačné doklady těžby stř́ibra dosud chybí (Militký $2015 a, 157)$. Ani v okolních regionech nejsou zatím přímé doklady těžby stříbra přiliš průkazné. Pouze nepřímé náznaky těžby pocházejí z hornatých regionů severního Slovenska (Pieta 2008, 74) a navíc je většina slovenského ložiskového stříbra obsažena v polymetalických rudách.

Zcela speciální kategorii představují stříbrné tyčinkovité ingoty. Ukazuje se, že zvláště v oppidálním období (LT D) je tato kategorie nálezů velice důležitá a dokládá intenzivní obchod se stř́ibrem ve standardizované podobě. Ve vztahu k bratislavskému mincovnictví známe jeden mimořádně zajímavý stř́ibrný ingot (Militký 2013a, 101,103 , č. 15 , obr. 2: 15), který byl údajně součástí depotu tetradrachem skupiny Biatec (Militký 2013a, 100-103, č. 1-14, obr. 1-4) pocházejícího z neznámé lokality. Jde o tyčinkovitý stříbrný ingot obdélného tvaru a lichoběžníkovitého prưřezu, který byl odlit do otevřené formy (obr. 6). Jeho hmotnost 418,184 g neodpovídá žádné známé antické hmotnostní jednotce. U předmětu byla provedena v Mnichově XRF nedestruktivní analýza složení kovu - zjištěná ryzost je okolo 93-94 \% Ag s příměsí mědi a cínu. Složení hřivny nelze konfrontovat se složením bratislavských mincí, nebot doposud bylo analyzováno jen zcela nepatrné množství ražeb.

Bratislavské oppidum s jistotou nekontrolovalo žádné lokální zdroje drahých kovů, nebot se ani v jeho širokém okolí nenacházejí. Je tedy prakticky jisté, že zlato i stříbro muselo být získáváno především obchodem. Lze jen spekulovat, odkud dovážené stříbro pocházelo - v úvahu přicházejí severoslovenské zdroje, ložiska v Sedmihradsku, ale také stř́ibro z Římské říše (Militký 2013a, 117). Právě tato varianta se dnes jeví jako velice lákavá.

\section{Numismatická analýza depotu Deutsch Jahrndorf}

\subsection{Zlaté mince}

Zcela jedinečné svědectví poskytují v pokladu zastoupené zlaté mince. Pro pochopení jejich významu je tř̌eba v širších souvislos- tech sledovat vývoj produkce zlatých nominálů oppidálního období (LT D) v bójské oblasti, tedy především na území Čech a Moravy. Tato probleamtika byla obsáhle zpracována v souvislosti s analýzou mincovních nálezů z oppida Stradonice (Militký 2015a). Právě $\mathrm{k}$ těmto bójským ražbám se bratislavské zlaté mince zřetelně hlásí.

Typologicky značně rozsáhlou skupinu zlatých ražeb produkovaných v Bratislavě reprezentují beznápisové statéry. Jejich největší typologické spektrum poskytl právě poklad Deutsch Jahrndorf, ze kterého pochází 18 exemplářù. Naopak v nálezu Bratislava 2009 byly zastoupeny pouze dva beznápisové statéry (Musilová Kolníková-Hložek 2015, 253-255, obr. 13: 14-15). Téma typologie beznápisových statérů je poměrně obsáhlé - v rámci tohoto příspěvku se omezujeme na analýzu exemplářů ze sledovaného depotu. Teprve budoucí komplexní zpracování problematiky umožní zasadit tyto exempláře do celé typologické škály těchto ražeb. Společný výskyt $\mathrm{v}$ pokladech $\mathrm{s}$ bratislavskými stř́ibrnými nominály a jejich shodné hmotnosti, jen vzácně převyšující 6,5 g, zřetelně naznačují paralelní produkci se statéry s nápisy BIATEC a NONNOS. Rozhodně je třeba odmítnout představu, že by beznápisové statéry představovaly starší horizont ražby na bratislavském oppidu již na přelomu 2. a 1. století před Kristem, a nebo, že jde o importované ražby z Čech či Moravy (Musilová - Kolníková - Hložek 2015, 273).

$\mathrm{V}$ rámci sledovaného pokladu se podařilo definovat celkem sedm skupin beznápisových statérů (tab. 3; kat. č. 1-18; obr. 4a: 1-10; 4b: 11-18). Jejich vzájemné chronologické vztahy jsou ovšem zatím nejasné. Ve všech případech jde o mušlový typ, pro který je typický hrbol na líci a mušlový motiv na rubu - jedná se o půlměsíc s paprsky na protilehlé ploše. Někdy je tento motiv považován za solárně-lunární symboliku a tuto možnost může podporovat i předpokládané zobrazení fází měsíce na líci varianty VI.1 (kat. č. 17). Označování statérů jako mušlový typ je tedy třeba vnímat pouze jako tradiční označení, které však nevyjadřuje motivickou podstatu rubního obrazu.

Rozbor beznápisových statérů z depotu ukazuje, že jejich ikonografie navazuje na ražby skupin Castelin (1965) C 13-15, produkovaných v Čechách, zvláště na oppidu Stradonice (Militký 2015a, 196-203, č. 57-74), v relativně-chronologickém úseku ražby C. Podle tradičního konceptu byla ražba zlatých mincí v Čechách na konci úseku $\mathrm{C}$ ukončena a přesunula se na oppidum Bratislava, kde reprezentuje relativně-chronologický úsek ražby D (Castelin 1965, 15-16, var. D 16-19). Komplexní zpracování materiálu z oppida Stradonice však jasně prokázalo, že konec ražby zlatých mincí v Čechách je souběžný, alespoň částečně, s existencí bratislavského oppida (Militký 2015a,49) a definice statérů úseků C a D je třeba korigovat. Je totiž jasné, že úsek D zahrnuje i českou produkci, jak dokládají exempláře $z$ depotu Podmokly a $z$ oppida Stradonice (Paulsen 1933, Taf. 18: 386-387, 19: 394-399; Castelin 1965, 15, var. C 14-15; Militký 2015a, 202-203, č. 75-78). Toto plně dokládají i vazby doložené exempláři z depotu Deutsch Jahrndorf, zvláště pak statér skupiny V (tab. 3: 16; kat. č. 16; Paulsen 1933, Taf. 18: 387), u kterého nelze zatím stanovit, jestli jde o minci bratislavskou či českou. Teprve budoucí komplexní analýza všech pozdních statérů relativně-chronologického úseku ražby $\mathrm{D}$ musí doložit, zda tyto mince nebyly produkovány i na dalších místech ve středním Podunají, tedy i mimo areál bratislavského oppida.

Mezi zlatými ražbami z pokladu Deutsch Jahrndorf jsou zastoupeny rovněž čtyři 1/3 statérů mušlové řady (tab. 3: 19-22; kat. č. 19-22). Tyto nominály byly komplexně zpracovány v rámci zpracování mincí z oppida Stradonice a zde byla zveřejněna i jejich nová typologie (Militký 2015a, 63-69, TAB. 1-4). Jde o nominál kontinuálně produkovaný po celé oppidální období v Čechách a do jisté míry zřejmě i na Moravě (Militký 2015a, 49-55, 57). Na líci těchto ražeb je obvykle hladký hrbol, na rubu jsou dva oválné hrboly obklopené klikatkou. V rámci tohoto nominálu bylo definováno šest základních typů: A-F (Militký 2015a, 63-69, TAB. 1-4). Jejich analýza prokázala, že typy A-E byly raženy postupně v Čechách, typ $\mathrm{E}$ i na Slovensku a typ F jen v Bratislavě (Militký 2015a, 54, tab. 10). Je zřejmé, že skupina $\mathrm{E}$ je v Čechách nejmladší a do tohoto horizontu spadají také dva exempláře $z$ depotu ze slovenského oppida Trenčianské Bohuslavice (Kolníková 1998, 12-14, obr. 1: 4-5). Nejmladší skupina F souvisí již pouze s horizontem bratislavského 
oppida. Je ovšem třeba zdůraznit, že nálezově ověřené exempláře 1/3 statérů skupiny $\mathrm{F}$ známe pouze ze sledovaného depotu (tab. 3: 20-21; kat. č. 20-21). Jedním exemplářem (tab. 3: 19; kat. č. 19) je v depotu Deutsch Jahrndorf zastoupena 1/3 statéru varianty Militký (2015a) E/VI:02 a dvěma exempláři (tab. 3: 20-21; kat č. 20-21) jsou zde zastoupeny 1/3 statérů varianty Militký (2015a) F/VII:01

Mezi zlatými ražbami z pokladu Deutsch Jahrndorf je zastoupena rovněž jedna beznápisová $\mathbf{1} / \mathbf{8}$ statéru mušlové řady ( $t a b$. 3 23; kat. č. 23). Také tyto nominály byly nově typologicky zpracovány v rámci publikace mincí z oppida Stradonice (Militký $2015 a$ 70-74, TAB. 5-7). Pro $1 / 8$ statérů se podařilo sestavit také jejich relativní chronologii, přičemž skupiny XII-XIV jsou s jistotou nejmladší (Militký 2015a, 59, tab. 14). Skupinu XIV reprezentuj exempláře s nápisem BIAT. Bez nápisové 1 /8 statérů reprezentuje skupina XIII (Militký 2015a, 74, TAB. 7). V depotu Deutsch Jahrndorf byl zastoupen pouze jediný exemplář $1 / 8$ statéru varianty $M i-$ litký (2015a) XIII:01.

Druhou skupinu zlatých ražeb z pokladu Deutsch Jahrndorf reprezentují statéry a jejich díly s nápisy. Se jménem BIATEC známe $1 / 3$ a $1 / 8$ statérů, se jménem NONNOS je zatím doložen pouze statér (Musilová - Kolníková - Hložek 2015, 255, obr. 13: 1013; obr. $7 a-7 b)$. Většina dosavadních informací o mincích se jménem BIATEC (či zkráceně BIAT) byla do nedávné doby známa především díky pokladu $z$ Deutsch Jahrndorfu a několika dalším exemplářum - jednomu z Parndorfu v Burgenlandu a třem dalším mincím bez známé nálezové lokality, které zveřejnil R. Paulsen (1933, Taf. 29: 695-705, 709-710). Zcela zásadně pozměnil pohled na tyto ražby depot $z$ římské stavby I na akropoli bratislavského oppida. V tomto nálezu bylo zastoupeno dalších devět statérů se jménem BIATEC K nim přistoupily také dva nedávno podchycené exempláře ze Slovenského Grobu (tab. 4: 17; obr. $7 b: 17$ ) a Chorvátskeho Grobu (tab. 4: 18). Celkem tak v současné době registrujeme 18 spolehlivě doložitelných exemplářů statérů se jménem BIATEC. Prostřednictvím fotografické dokumentace se podařilo sestavit zcela novou typologii těchto ražeb (tab. 4: 1-18; obr. 7a: 1-10, 7b: 12-17). Vysoká variabilita těchto statérů s nápisem BIATEC je zajímavá i s ohledem na poměrně malý počet doložených exemplářů. Drtivá většina statérů leží hmotnostně v intervalu 6,5-6,4 g. Analýzy složení kovu byly provedeny pouze $u$ exemplářu $z$ bratislavského depotu a jejich obsahy zlata se pohybují v rozmezí 88-84 \% (Musilová - Kolníková Hložek 2015, 283-284, tab. 7-8). V rámci úplnosti typologie bratislavských zlatých mincí s nápisy uvádíme na tomto místě také čtyři statéry s nápisem NONNOS, doložené zatím pouze v pokladu Bratislava 2009 (tab. 4: 19-22; obr. 7b: 19-22).

Se jménem Biatec se ve zkrácené formě setkáváme také na 1/3 statérů mušlové řady s nápisem BIAT. $Z$ ikonografického hlediska jde stále o ražby navazující na emise produkované po celé oppidální období v Čechách a do jisté míry zřejmě i na Moravě ( $M i$ litký 2015a, 63-66, TAB. 1-4). Jedna z nich byla zastoupena i v depotu Deutsch Jahrndorf (tab. 3: 27; kat. č. 27). Mezi zlatými ražbami $\mathrm{z}$ tohoto pokladu jsou zastoupeny také dvě 1/8 statérů mušlové rady s nápisem BIAT (tab. 3: 28-29; kat. č. 28-29). $\mathrm{V}$ rámci nové typologie $1 / 8$ statérů jde o skupinu XIV reprezentovanou jednou variantou (Militký 2015a, 72, TAB. 7), která byla zřejmě ražena paralelně s výše uvedenou beznápisovou skupinou XIII.

Zlaté beznápisové i nápisové nominály produkované na bratislavském oppidu jsou tedy ražby, které jednoznačně odrážejí vazby do českého pozdně oppidálního prostředí a přinejmenším v rámci zažité numismatické terminologie je lze považovat za ražby bójské. Tato zjevná souvislost stála za představou o odchodu keltských elit z českých zemí do středního Podunají (např. Waldhauser 1983. 335-336; Kolníková 1991, 21). Tento předpoklad má jistě své logické opodstatnění a nelze tvrdit, že k odchodu nedošlo, ale ukazuje se, že případný transfer obyvatelstva rozhodně v Čechách nezpůsobil úpadek osídlení, jak se někdy soudilo. Z numismatického pohledu je také zjevné, že ani v závěrečném období ražby D nedošlo v Čechách $\mathrm{k}$ přerušení produkce zlatých a stříbrných mincí. Dokládá to shodná ikonografie i hmotnosti zlatých nominálů, především u statérů, produkovaných paralelně v Bratislavě i v Čechách, především na oppidu Stradonice (Militký 2015a, 49).

\subsection{Střibrné mince}

V depotu Deutsch Jahrndorf bylo zastoupeno nejméně 134 tetradrachem skupiny Biatec (tab. 5; obr. $4 c-4 i)$. Drtivá většina těchto mincí je fyzicky dochována, nebo byla v literatuře alespoň vyobrazena. $\mathrm{V}$ pokladu byly zastoupeny mince se jmény AINORIX, BIATEC, BVSSVMARVS, BVSV, COBROVOMARVS, COISA, COVIOMARVS, DEVIL (nebo DEVII), EVOIVRIX (nebo FAPIARIX), LATVMARUS (IANTVMARVS), NONNOS a TITTO. Nejsou zde zastoupena pouze jména COVNOS (Göbl 1994, var. VIII/1-15), FARIARIX / nebo FAPIARIX/ (Göbl 1994, var. XI/1-27), LATTV (Militký 2013b, 162, č. 21-22, obr. 1c: 21-22; 2014), MACCIVS (Göbl 1994, var. XIII/1-22) a anonymní typ (Göbl 1994, var. XVI/1-17). Chybí zde také vzácné varianty s nápisy BIATEC, EVOIVRIX (nebo FAPIARIX) a hybrid BVSVMARVS+TITTO (tab. 5). Ikonografické vazby těchto ražeb na římské republikánské denáry jsou shrnuty v tab. 2 . Také $\mathrm{z}$ pohledu zastoupených kombinací razidel je zde prrítomna jejich naprostá většina (tab. 6; obr. 15-16). Je tedy zřejmé, že společně s pokladem z Bratislavy z roku 1942 (Ondrouch 1958) nález z Deutsch Jahrndorfu představuje druhý nejvýznamnější nálezový soubor obsahující tetradrachmy skupiny Biatec.

Jedním exemplářem (kat. č. 30) je v depotu zastoupena tetradrachma s nápisem AINORIX Göbl (1994) I/1-26. Ikonografie této ražby je specificky keltská, avšak motiv Harpie se odvolává k antické mytologii. Na líci je zobrazeno mladistvé mužské (?) poprsí, na rubu je stojící Harpyie doprava. Obrazově je tato ražba prakticky identická s tetradrachmou s nápisem TITTO (Göbl 1994, XV/1-25; kat. č. 155-156) a FARIARIX /nebo FAPIARIX/ (Göbl 1994, XI/1-27). Je zřejmé, že všechny tři typy vznikly přibližně ve stejné době.

Druhou nejpočetnější skupinu (49 ks) ve sledovaném depotu představují tetradrachmy s nápisem BIATEC (kat. č. 31-79). Je zde př́tomna většina doložených kombinací razidel (tab. 6). V rámci úrovně publikovaného materiálu jsou zde poprvé prezentovány i nově definované varianty a to v rámci R. Göblem (Göbl 1994) vyčleněných kombinací razidel II/1(1)-1 a II/2 (2)-6. Celkem šesti exempláři (kat. č. 31-36) jsou ve sledovaném pokladu zastoupeny tetradrachmy nově definované varianty Röttger (2019) II/1(1)B-1B s hlavami Honos a Virtus na líci - jde o motiv převzatý z římských republikánských denárů a setkáváme se s ním na většině známých variant se jménem BIATEC. Na rubu je umístěn motiv jezdce v různých variacích. Celkem 11 exempláři (kat. č. 37-46) jsou ve sledovaném pokladu zastoupeny tetradrachmy nově definované varianty Röttger (2019) II/1 (1)B-1C. Třemi exempláři (kat. č. 47-49) jsou v depotu zastoupeny tetradrachmy varianty Göbl (1994) II/1 (2/1)-2, šesti exempláři (kat. č. 50-55) tetradrachmy varianty Göbl (1994) II/1 (2/2)-3, 12 exempláři (kat. č. 56-67) tetradrachmy varianty Göbl (1994) II/1 (3)-4 a pěti exempláři (kat. č. 68-72) jsou v pokladu zastoupeny tetradrachmy nově definované varianty Röttger (2019) II/2 (2)B-6B. Dvěma exempláři (kat. č. 73-74) jsou zastoupeny tetradrachmy varianty Göbl (1994) II/3-12 - mimořádně zajímavá je její rubní strana. Je zde zobrazen do půl těla nahý (?) jezdec v kalhotách na osedlaném koni doprava držící v pravé ruce meč, v levé ruce velký oválný štít a uzdu (?). Přes stylizaci kresby je na vyobrazení jasně zřetelné, že kưn̆ je osedlaný a na těle koně jsou také řemeny použité $\mathrm{k}$ upevnění sedla. S prakticky identickým rubem se setkáváme rovněž u britské bronzové mince nápisem VER (Röttger 2015, obr. 7). Tato ražba je datována do let asi 25 před Kristem až 10 po Kristu (Cottam et al. 2010, 131, č. ABC 2676). Je značně pravděpodobné, že předlohou pro britskou ražbu byly bratislavské tetradrachmy. Jediným exemplářem (kat. č. 75) je v pokladu zastoupena tetradrachma varianty Göbl (1994) II/4-3. Oproti předchozím variantám jde o ražbu odlišné ikonografie - na líci je zobrazena mužská hlava s krátkými vlasy doprava. Čtyřmi exempláři (kat. č. 76-79) jsou zastoupeny tetradrachmy varianty Göbl (1994) II/4-7 - ikonografie této ražby je shodná s předchozí variantou.

Dvěma exempláři (kat. č. 80-81) jsou ve sledovaném depotu zastoupeny tetradrachmy s nápisem BVSSVMARVS typu Göbl (1994) III/1-24. Ikonografie této ražby se odvolává $\mathrm{k}$ antickým předlohám. Na lícní straně je mužské (?) poprsí, na rubu je zobrazen gryf. Ražba je krásnou ukázkou volného převzetí antických předloh prezentovaných v lokálním keltském výtvarném stylu.

Pěti exempláři (kat. č. 82-86) jsou v depotu zastoupeny tetradrachmy s nápisem BVSV varianty Göbl (1994) IV/1-28. I v tomto 
případě se ikonografie odvolává $\mathrm{k}$ antickým předlohám. Na lícní straně je opět zobrazeno mužské (?) poprsí, na rubní straně je zobrazen okřídlený kentaur (?) s ženskou polopostavou. I v tomto př́i padě jde o jedinečnou ukázku transformace antických předloh v lokálním keltském výtvarném stylu.

Dvěma exempláři (kat. č. 87-88) jsou ve sledovaném depotu zastoupeny tetradrachmy s nápisem COBROVOMARVS varianty Göbl (1994) V/1-18. Na lícní straně je zobrazena mužská a ženská hlava doprava (Honos a Virtus), na rubu je zobrazen lev ve skoku doprava. Charakter ztvárnění rubního motivu je velmi podobný tetradrachmám s nápisem BVSSVMARVS (Göbl 1994, III/1-24).

Jedním exemplářem (kat. č. 89) je v depotu zastoupena tetradrachma s nápisem COISA varianty Göbl (1994) VI/1-21. Ikonografie této ražby je kombinací římské předlohy a specifické keltské invence. Na líci je mužská hlava $\mathrm{v}$ přilbě (nebo čepici) doleva, rubní motiv je specificky keltský - je zde prezentován stojící muž v krátkém oděvu doprava bojující se stojícím medvědem (?) nebo kancem (?).

Třemi exempláři (kat. č. 90-92) jsou ve sledovaném depotu zastoupeny tetradrachmy s nápisem CovioMARVS varianty Göb (1994) VII/1-23. Ikonografie této ražby je opět specificky keltská. Na lícní straně je mladistvá mužská hlava doprava, na rubu je zobrazen stylizovaný stojící kanec doleva.

Třemi exempláři (kat. č. 93-95) jsou v pokladu zastoupeny tetradrachmy s nápisem DEVIL varianty Göbl (1994) IX/1-16. Na líci je zobrazena mužská hlava s krátkými vlasy doprava. Hlava je ztvárněna ve specifickém keltském stylu, ovšem jde zřetelně o inspiraci v římském pozdně republikánském mincovnictví. Na rubu je zobrazen kůn̆ ve skoku doprava. Pravděpodobně třemi exempláři (kat. č. 96-98) jsou v depotu zastoupeny tetradrachmy s nápisem DEVIL varianty Göbl (1994) IX/1-20. Líc těchto mincí je ražen stejným razidlem jako předchozí varianta, motivem rubní strany je stojící medvěd. Ch. Röttger (2013, 34-36) navrhl čtení nápisů DEVIL jako DEVII.

Jediným exemplářem (kat. č. 99) je v pokladu zastoupena tetradrachma s nápisem EVOIVRIX varianty Göbl (1994) X/2-19. Na lícní straně je zobrazeno mladistvé mužské poprsí, na rubu je lev ve skoku doleva - motiv je evidentně opět inspirován antickými předlohami. Ch. Röttger (2013, 37-38) navrhl čtení nápisu EVOIVRIX jako zrcadlově otočený nápis FAPIARIX. Tato interpretace však zůstává otevřená a čtení nápisu jako EVOIVRIX se jeví jako pravděpodobnější.

Čtyřmi exempláři (kat. č. 100-103) jsou ve sledovaném pokladu zastoupeny tetradrachmy $\mathrm{s}$ nápisem LATVMARVS typu Göbl (1994) XII/1-14. Tato ražba byla tradičně čtena jako IANTVMARVS s ligaturou ANT. Při analýze sledovaného depotu se však jednoznačně ukázalo, že toto čtení není správné. Nabízí se totiž daleko jednodušší možnost čtení jako LATVMARVS, přičemž písmena LATV jsou jasně čitelná na dobře zachovaném exempláři, který však nepochází ze sledovaného pokladu (obr. 13: 1). Navíc tak tato mince navazuje na nedávno zveřejnený nový typ s nápisem LATTV (Militký 2013b, 166, obr. 1c; 2014; obr. 13: 2), pocházející z depotu odkrytého v okolí Salzburgu (Militký 2013b). Ukazuje se tak, že obě mince jsou zřetelně spojeny s bratislavským oppidem. Jejich výrazně odlišný styl však naznačuje, že zřejmě nebyly raženy ve stejné době a tetradrachma s nápisem LATTV je možná vůbec nejmladší emisí skupiny Biatec (Militký 2013b, 173). Nevíme také s jistotou, jestli jde o stejnou osobu, nicméně rozdílnost psaní jména s jedním či dvěma T nemusí naznačovat dvě odoby, podobně jako u jméma BVSV a BVSSVMARVS (kat. č. 80-86; obr. 4e: 80-85). Ch. Röttger (2013) nedávno navrhl nová čtení jmen DEVIL jako DEVII, FARIARIX jako FAPIARIX a EVOIVRIX jako zrcadlově otočenou formu jména FARIARIX nebo FAPIARIX - tyto reinterpretace nápisů jsou jistě zajímavé, ale nikoliv jisté. Nopak nápis LATVMARVS je čitelný zcela zřetelně a v kontextu nápisu LATTV je jeho nové čtení význámým posunem v chápání jmen na bratislavských tetradrachmách. Ikonografie této ražby je keltská. Na líci je zobrazeno mladistvé poprsí, na rubu je jezdec na koni.

Vůbec nejpočetněji (51 ks) jsou ve sledovaném depotu zastoupeny tetradrachmy s nápisem NONNOS (kat. č. 104-154). Jsou zde přítomny prakticky všechny známé kombinace razidel s výjimkou velmi vzácné varianty Göbl (1994) XIV/1B-10 (tab. 6). Celkem 18 exempláři (kat. č. 104-121) jsou v pokladu zastoupeny tetradrachmy varianty Göbl (1994) XIV/1A-8. Ikonografie této ražby je kombinací římské republikánské předlohy a specifického keltského výtvarného projevu. Na lícní straně je zobrazena ženská hlava, na rubu je jezdec na koni. Celkem 12 exempláři (kat. č. 122-133) jsou ve sledovaném pokladu zastoupeny tetradrachmy varianty Göbl (1994) XIV/1B-9. Ikonografie tohoto typu mince je opět kombinací římské republikánské předlohy a keltského výtvarného projevu. Na lícní straně je zobrazena ženská hlava, na rubu je jezdec na koni. Třemi exempláři (kat. č. 134-136) jsou v depotu zastoupeny tetradrachmy varianty Göbl (1994) XIV/1C-10. I v tomto případě je ikonografie tohoto typu mince kombinací římské republikánské předlohy a specifického keltského výtvarného projevu. Na lícní straně je zobrazeno ženské poprsí, na rubu je zobrazen jezdec na koni. Třemi exempláři (kat. č. 137-139) jsou ve sledovaném pokladu zastoupeny tetradrachmy s nápisem NONNOS varianty Göbl (1994) XIV/3-11. Na líci je zobrazeno mužské poprsí, na rubu je zobrazen jezdec na koni. Celkem 14 exempláři (kat. č. 140-153) jsou v nálezu zastoupeny tetradrachmy varianty Göbl (1994) XIV/2 (1)-13. Sledovaná varianta představuje př́klad specificky keltské ikonografie. Na líci je zobrazena mužská hlava s krátkými vlasy, na rubu je jezdec na koni držící v meč a oválný štít. Výrazným prvkem rubní strany je zrcadlově obrácený nápis NONNOS. Na vyobrazení jezdce je zajímavý detail - opasek se svisle visící pochvou meče.

Poslední typ tetradrachem zastoupený v depotu Deutsch Jahrndorf reprezentují dva exempláře (kat. č. 155-156) tetradrachem s nápisem TITTO varianty Göbl (1994) XV/1-25. Ikonografie této mince je specificky keltská, avšak motiv Harpie se odvolává k antické mytologii. Na líci je zobrazeno mladistvé mužské (?) poprsí doleva, na rubu je zobrazena stojící Harpyie doprava, hledící doleva. Obrazově je sledovaná ražba prakticky shodná s tetradrachmami s nápisem AINORIX (Göbl 1994, var. I/1-26; kat. č. 31) a FARIARIX (Göbl 1994, var. XI/1-27) - je zřejmé, že všechny tři typy byly raženy přibližně ve stejné době.

\section{Dobová hodnota, stanovení doby ukrytí a významu depotu}

Z dobového hlediska představuje poklad z Deutsch Jahrndorfu, resp. jeho dochovaná část, sumu odpovídající necelým 250 tetradrachmám - tedy při akceptování římského poměru ceny zlata a stříbra $1: 12,5$, při kterém odpovídá jeden statér zhruba pěti tetradrachmám. Jde tak o druhý největší peněžní depozit bratislavských keltských mincí. Největším z nich stále zůstává poklad z Bratislavy z roku 1942, který obsahoval 270 tetradrachem (Ondrouch 1942). Víme, že sledovaný poklad byl ukryt v nedochované keramické nádobě, podobně jako několik dalších depotů obsahujících tetradrachmy tytu Biatec.

Zásadní otázkou je nálezové prostředí pokladu. Bohužel zatím nevíme, jestli byl poklad ukryt v areálu dobově osídlené plochy, nebo ve volné neosídlené krajině. V každém případě však nález souvisí $\mathrm{s}$ předpokládanou komunikací směřující na jih od bratislavského oppida - místo nálezu je vzdáleno ideálně jižně $15 \mathrm{~km}$ od jeho akropole. $Z$ oblastí proti proudu Dunaje či z prostoru směrem k Aplám známe několik pokladů (Wien-Simmering, Swechat, Salzburg - okolí; obr. 5) i jednotlivých nálezů. K těmto souborům přibyl nově identifikovaný a dosud nepublikovaný poklad Potzneusiedl. Tyto nálezy zřejmě souvisejí se zájmovou či př́mo ovládanou zónou bratislavského oppida a v případě alpských nálezů pak s využíváním komunikačního spojení mezi Bratislavou a Římským impériem. V této souvislosti bude nutné zároveň řešit vztah některých nálezů (Wien-Simmering a Swechat; obr. 5) k soudobému rovinnému sídlišti na místě dnešní Vídně s mincovní výrobou doloženou prostřednictvím tavících destiček (Adler-Wölfl 2012; Adler-Wölfl Mosser 2015). Poklad z Deutsch Jahrndorfu, společně s pokladem Potzneusiedl, však spadá zřejmě do zóny přímo ovládané $z$ Bratislavy. Je zároveň velice pravděpodobné, že sledovaná peněžní hotovost byla akumulována př́mo v areálu bratislavského oppida.

S otázkou výše řešené chronologie bratislavské mincovní produkce výrazně souvisí také snaha o stanovení doby ukrytí depotu z Deutsch Jahrndorfu. S ohledem na zastoupení většiny variant tetradrachem (tab. 6) je možné uvažovat o době blízké úpadku či přímo zániku oppida. Důležitá je v této souvislosti absence tetradrachmy s nápisem LATTV, která je zřejmě vưbec nejmladší nápi- 
sovou emisí (Militký 2013b, 173). Avšak absence některých typů mincí nemusí být nutně jen chronologickým indikátorem. Za stávajících chronologických znalostí lze tedy jako o době ukrytí pokladu z Deutsch Jahrndorfu uvažovat o 3. čtvrtině 1 . století před Kristem.

Otevřenou a s jistotou nezodpověditelnou otázkou je kmenová příslušnost emitentů mincí pokladu z Deutsch Jahrndorfu, tedy elit bratislavského oppida. Tradičně jsou spojovány s Bóji, předpokládaně usazenými v Čechách (Militký 2015a, 169). Bójský element v Bratislavě v každém případě reprezentují zlaté nominály a stříbrné oboly typu Karlstein, naopak tetradrachmy skupiny Biatec a hemidrachmy typu Simmering jsou zcela specifické lokální nominály, které nemají v keltském prostředí obdoby. Zjevná dominance těžkých stříbrných mincí jasně ukazuje na pomezí bimetalických peněžních systémů, typických pro bójské prostředí a monometalického stříbrného východokeltského světa (Militký - Karwowski 2013 26). Mince samozřejmě nemohou jednoznačně odpovědět na otázku kmenové příslušnosti, v případě Bratislavy však jasně ukazují na velice úzkou provázanost této lokality s českým prostředím a tedy předpokládaně i s Bóji (Militký 2015b, 93-94).

Poklad z Deutsch Jahrndorfu představuje zcela jedinečný pramen k poznání bratislavské mincovní produkce. Jeho skutečný význam nebyl po mnoho desetiletí plně zjevný. Zpracováním tohoto souboru se výrazným způsobem podařilo prohloubit poznání bratislavských keltských mincí. Nové archeologické nálezy římské architektury na akropoli bratislavského oppida navíc umožňují lépe pochopit fenomén tamních vztahů mezi Rímskou republikou a lokálními keltskými elitami. K tomu tématu se snaží pohledem numismatiky přispět také detailní zpracování sledovaného depotu.

\section{Bibliography}

Adler-Wölfl, K. 2012:

Spätlatènezeitliche Fundstellen in Wien. Zborník Slovenského národného múzea 106 - Archeológia 22, 169-188.

Adler-Wölfl, K. - Mosser, M. 2015:

Archäologie am Rochusmarkt - Die Grabungen in Wien 3, Rasumofskygasse 29-31. Fundort Wien. Berichte zur Archäologie $18,4-48$.

Alföldi, A. 1942:

Zur Geschichte des Karpatenbeckens im 1. Jahrhundert v. Chr. Budapest.

Almgren, O. 1923:

Studien über Nordeuropäische Fibelformen. Leipzig.

Allen, D. F. 1972:

Celtic Coins in the Royal Coin Cabinet, Stockholm. Nordisk Numismatisk Årsskrift 1972, 5-26.

Allen, D. F. 1987:

Catalogue of the Celtic Coins in the British Museum with Supplementary Material from other British Collections. Volume I. Silver Coins of the East Celts and Balkan Peoples. London.

Anonym 1905:

Münzfunde. Blätter für Münzfreunde 40, 3317.

Bíró-Sey, K. 1987:

Celtic Coins of the Danube Region in West Swedish Collections. Bohusgruppen.

Boczek, F. 1840:

Beiträge zu Mährens Münzen aus dem Mittelalter. (Zweiter Aufsatz, über slawische Gold- und Silber-Münzen). Mittheilungen der k. k. Mährisch-Schlesischen Gesellschaft zur Beförderung des Ackerbaues, der Natur- und Landeskunde in Brünn 38, 180-183, 186-191.

Budaj, M. - Čambal, R. 2013a:

Keltské mince a zlatý ingot zo Slovenského Grobu - Celtic coins and golden ingot from Slovenský Grob. Pravěk Nová řada 21, 209-222.
Budaj, M. - Čambal, R. 2013b:

Drachmy typu Simmering s nápisom NONNOS zo Slovenska - Simmering type drachms with the inscriptions NONNOS from Slovakia. Pravěk Nová řada 21, 223-233.

Budaj, M. - Čambal, R. 2016:

Laténské nálezy z Bratislavy - Devínskej Kobyly — La Tène objects from Bratislava - Devínska Kobyla. Numismatický sborník 30/2, 273-283.

Button 95:

Frankfurter Münzhandlung E. Button Auktionskatalog Nr. 95. 1955. Frankfurt am Main.

Button 107:

Frankfurter Münzhandlung E. Button Auktionskatalog Nr. 107. Januar 1963. Frankfurt am Main.

Castelin, K. 1965:

Die Goldprägung der Kelten in den böhmischen Ländern. Graz.

Castelin, K. 1978:

Keltische Münzen. Katalog der Sammlung im Schweizerischen Landesmuseum Zürich. Band I. Zürich.

Chabouillet, M. A. 1889:

Catalogue des monnaies gauloises de la Bibliothèque nationale. Paris.

Cottam et al. 2010:

Cottam, E. - de Jersey, Ph. - Rudd, Ch. - Sills, J.:

Ancient British Coins. Aylsham.

Čambal, R. 2004:

Bratislavský hradný vrch - akropola neskorolaténského oppida / Der Bratislavaer Burghügel - die Akropolis von Spätlatènezeitlichem Oppidum. Zborník Slovenského národného múzea, Archeológia, Supplementum 1. Bratislava.

Čambal, R. - Budaj, M. 2009:

Keltské tetradrachmy z Pezinka a Bratislavy-Rače - Keltische Tetradrachmen aus Pezinok und Bratislava-Rača. Zborník Slovenského národného múzea 103 - Archeológia 19, 197-214.

De La Tour, H. 1892:

Atlas de monnaies gauloises. Paris.

Delestrée, L.-P. - Tache, M. 2002:

Nouvel atlas des monnaies gaulouises I. De la Seine au Rhin. Saint-Germain-en-Laye.

Dembski, G. 1998:

Münzen der Kelten. Kataloge der antiken Münzen, Reihe A: Griechen II: Massalia und Keltenstämme. Sammlungskataloge des Kunsthistorischen Museums, Band 1. Wien.

Dembski, G. 1999:

Münze oder Ware? Keltische Hackmünzen, Barren und Schrötlinge aus österreichischen Fundorten. In: Bertók, K. - Torbágyi, M. /eds./: Festschrift für Katalin Bíró-Sey und István Gedai zum 65. Geburtstag. Budapest, 107-122.

Dembski, G. 2009:

Eigenprägung und Fremdgeld - Die Fundmünzen aus Roseldorf. In: V. Holzer /ed./: Roseldorf. Interdisziplinäre Forschungen zur größten keltischen Zentralisierung Österreichs. Schriftenreihe der Forschung im Verbund, Band 102. Wien, 88-101.

Depeyrot, G. 2002:

Le numéraire celtique I. La Gaule du Sud-Est. Collection Moneta 27 . Wetteren.

Depeyrot, G. 2005:

Le numéraire celtique VII. La Gaule orientale. Collection Moneta 46. Wetteren.

Dessewffy, M. 1910:

Barbár Pénzei. Budapest. 
Dobesch, G. 1994:

Zur Chronologie des Dakerkönigs Burebista. In: Göbl, R. /ed./: Die Hexadrechmenprägung der Gross-Boier. Ablauf, Chronologie und historische Relevanz für Noricum und Nachbargebiete. Wien, 51-68.

Dobesch, G. 1995:

Die Boier und Burebista. In: Tejral, J. - Pieta, K. - Rajtár, J. /eds./: Kelten, Germanen, Römer im Mitteldonaugebiet vom Ausklang der Latène - Zivilisation bis zum 2. Jahrhundert. Brno - Nitra, 15-19.

Dobiáš, J. 1964:

Dějiny československého území před vystoupením Slovanů The History of the Czechoslovak Territory before the Apperace of the Slavs. Praha.

Eckhel, J. 1779:

Catalogus Musei Caesarei Vindobonensis Numorum Veterum. Pars I. Wien.

Egger 1898:

Sammlung Carl Leopold Latour v. Thurmburg. Wien: Brüder Egger. 21. 11. 1898.

Egger 7:

VII. Verkaufskatalog. Wien: Brüder Egger 1893-1894.

Egger 14:

XIV. Verkaufskatalog. Wien: Brüder Egger 1908.

Egger 15:

XV. Verkaufskatalog. Wien: Brüder Egger 1910.

Eisner, J. 1925:

Mince typu Biatec, nalezené v Bratislavě r. 1923. Numismatický časopis československý 1, 87-120.

Fiala, E. 1891:

Beschreibung böhmischer Münzen und Medailen. Prag.

Fiala, E. 1900:

Collection Ernst Prinz zu Windisch-Grätz. Anhang zu dem Band (V) Griechen: Die Prägungen der Barbaren. Prag.

Forrer, R. 1908:

Keltische Numismatik der Rhein- und Donaulande. Band 1. Graz.

FRMÖ I/2:

Dick, F. 1984:

Die Fundmünzen der römischen Zeit in Österreich. Abteilung I/2. Burgenland. Wien.

Fröhlich, J. 2011:

Unikátny keltský statér s nápisom DEVI[L]. K spoločenskohospodárskej organizácii stredodunajských Keltov - Unique Celtic stater with inscription of DEVI[L]. Contribution to the socialeconomic organization of the Celts in the middle-Danube region. Numismatický sborník 25, 3-22.

Fröhlich, J. 2012:

Drahý kov z moravského keltského centra Němčice - Precious metal from the Moravian Celtic centre in Němčice. Numismatické listy 67, 147-159.

Fröhlich, J. 2016:

Poklad keltských šperkov a mincí z kultovného miesta na kopci Žeravica pri obci Stupné na Slovensku - Depot von keltischem Schmuck und Münzen aus der Kultstätte auf dem Žeravica-Hügel in der nähe der Gemeinde Stupné. Folia numismatica 30/1, 3-24.

Gorini, G. 2009:

Norican silver coinage: a reappraisal. In: van Heesch, J. Heeren, I. /eds./: Coinage in the Iron Age. Essays in Honour of Simone Scheers. London, 117-122.
Göbl, R. 1973:

Typologie und Chronologie der keltischen Münzprägung in Noricum. Veröffentlichungen der Numismatischen Kommission, Band 2. Wien.

Göbl, R. 1994:

Die Hexadrachmenprägung der Gross-Boier. Ablauf, Chronologie und historische Relevanz für Noricum und Nachbargebiete. Wien.

Harmadyová, K. 2012:

Devín v rímskej dobe. In: Šedivý, J. - Štefanovičová, T. /eds./: Dejiny Bratislavy 1. Od počiatkov do prelomu 12. a 13. storočia. Bratislava, 271-275.

Haupt, P. - Nick, M. 1997:

Ein neuer Münzschatz mit Denaren der Eravisker aus der Gegend von Györ (Ungarn). Numismatische Zeitschrift 104-105, 41-81.

Humer, F. 2006:

Keltische Münzpragung in Bratislava. In: Humer, F. /ed./: Legionsadler und Druidenstab. Vom Legionslager zur Donaumetropole. Katalogband. Horn, 22-27.

Kellner, H.-J. 1990:

Die Münzfunde von Manching und die Keltischen Fundmünzen aus Südbayern. Die Ausgrabungen in Manching, Band 12. Stuttgart.

Kenner, F. 1880:

Fund keltischen Münzen in Simmering bei Wien. Numismatische Zeitschrift 12, 502-504.

Kenner, F. 1895:

Der Münzfund von Simmering in Wien. Numismatische Zeitschrift 27, 57-86.

Kerényi, A. 1959:

Gruppierung der Barbarenmünzen Transdanubiens. Folia Archaelogica 11, 47-60.

Kolníková, E. 1990:

Neznámy hromadný nález keltských mincí z okolia Modry alebo Jura pri Bratislave z roku 1910 - Unbekannter Hortfund keltischer Münzen aus der Umgebung von Modra oder Jur při Bratislave im Jahre 1910. Slovenská numizmatika 11, 249-253.

Kolníková, E. 1991:

Bratislavské keltské mince. Bratislava.

Kolníková, E. 1995:

Nezvyčajná keltská minca s nápisom BIATEC — Ungewöhnliche keltische Münze mit der Umschrift BIATEC. Numismatické listy 50, 1-7.

Kolníková, E. 1996:

Norische Münzen im Oppidum Bratislava und in seinem Hinterland. Slovenská numizmatika 14, 9-57.

Kolníková, E. 1998:

Výpoved' nálezov mincí o keltskom hradisku v Trenčinských Bohuslaviciach - Aussage der Münzfunde über den keltischen Burgwall in Trenčianske Bohuslavice. Slovenská numizmatika $15,11-44$

Kolníková, E. 2005:

Münzen der Eravisker, Azaler und andere Nachahmungen der Republikanischen Denare im Nordkarpatischen Raum. In: Bíró-Sey, K. - Buza, J. - Csoma, M. - Gedai, I. /eds./: In memoriam Ludovici Huszár. Budapest, 72-89.

Kolníková, E. 2012a:

Mince - svedectvo prosperity Keltov. In: Šedivý, J. - Štefanovičová, T. /eds. /: Dejiny Bratislavy 1. Od počiatkov do prelomu 12. a 13. storočia. Bratislava, 205-215. 
Kolníková, E. 2012b:

K technike a technológii v keltskom mincovníctve - nálezy zo Slovenska - Contribution to the technique and technology of the Celtic coinage - finds from Slovakia. Numismatický sborník 26/1, 31-57.

Kolníková, E. - Bakos, F. - Pauditš, P. 2018:

Zdroje kovu v keltských mincovniach na Slovensku — Sources of metal Celtic mints in Slovakia. Zborník Slovenského národného múzea 112 - Archeológia 28, 141-195.

Kolníková, E. - Kovár, B. 2010:

Laténske mince $\mathrm{v}$ bratislavskom podhradí - Latènezeitliche Münzfunde aus der bratislavaer Vorburg. Zborník Slovenského národného múzea 104 - Archeológia 20, 69-73.

Kostial, M. 2003:

Kelten im Osten. Gold und Silber der Kelten in Mittel- und Osteuropa. Sammlung Lanz. München ( $2^{\text {nd }}$ edition).

Kress, K. 91:

Auktion 91, München 1955.

Krmnicek, S. 2010:

Münze und Geld im frührömischen Ostalpenraum. Studien zum Münzumlauf und zur Funktion vom Münzgeld anhand der Funde und Befunde vom Magdalensberg. Klagenfurt am Wörthersee.

Kupido, F. S. 1866:

Die Silbermünzen des Jarendorfer Fundes. Wiener Numismatische Monatshefte 2, 98-106.

Kysela, J. - Olmer, F. 2014:

The Roman amphorae discovered in the excavation of the Bratislava castle - a preliminary study. In: Musilová, M. - Barta, P. - Herucová, A. /eds./: Bratislavský hrad: dejiny, výskum a obnova. Bratislava, 167-188.

Lesák, B. 2016:

Neskorolaténske osídlenie na nádvorí paláca Bratislavského hradu. - L'insediamento nel periodo di La Tène Tardo nello spazio del cortile del palazzo del castello di Bratislava. In: $\mathrm{Mu}$ silová, M. et al. /eds. /: Kelti z Bratislavy. Bratislava, 117-120.

Lukanc, I. 1996:

Les imitations des monnaies d'Alexandre le Grand et de Thasos. Wetteren.

Macho \& Chlapovič 9:

Aukčný dom Macho \& Chlapovič. Aukce 9, 26.-27. 10. 2015. Praha.

Macho \& Chlapovič 10:

Aukčný dom Macho \& Chlapovič. Aukce 10, 9.-10. 5. 2016. Bratislava.

Macho \& Chlapovič 12:

Aukčný dom Macho \& Chlapovič. Aukce 12, 7. 4. 2017. Praha.

Macho \& Chlapovič 18 :

Aukčný dom Macho \& Chlapovič. Aukce 18, 3. 5. 2019. Praha.

Militký, J. 2004:

Zapomenutý nález keltské mince typu AINORIX z Bratislavy Vergessener Fund einer keltischen Münze des Typs AINORIX aus Bratislava. Slovenská numizmatika 17, 189-190.

Militký, J. 2011 :

Die keltischen Münzen vom Oberleiserberg (Niederösterreich). In: Holmes, N. /ed./: Proceedings of the XIV th International Numismatic Congress, Glasgow 2009, II. Glasgow, 1198-1206.

Militký, J. 2013a:

Hromadný nález hexadrachem typu Biatec a stříbrného ingotu z neznámé lokality. K otázce původu stříbra u středoevropských Keltů - A group of hexadrachms of the Biatec type and a silver ingot from an unknown locality. Contribution to the origin of silver in the case of the central European Celts. Numismatické listy 68, 99-122.
Militký, J. 2013b:

Hromadný nález hexadrachem typu Biatec $z$ neznámé lokality a nový typ bratislavské mince s nápisem LATTV — A group find of the hexadrachms of the Biatec type from an unknown locality and a new coin type with LATTV inscription from Bratislava. Numismatický sborník 27/2 (2012-2013), 161-178.

Militký, J. 2014:

Ein neuer Hexadrachmentyp vom Typ Biatec mit der Aufschrift LATTV. Mitteilungen der Österreichischen Numismatischen Gesellschaft 54, 25-38.

Militký, J. 2015a:

Oppidum Hradiště u Stradonic. Komentovaný katalog mincovních nálezů a dokladů mincovní výroby — Das Oppidum Hradiště bei Stradonice. Kommentierter Katalog der Münzfunde und Belege der Münzproduktion. Praha.

Militký, J. 2015b:

Die Boier und die Numismatik - Gegenwärtiger Stand der Forschung und die Möglichkeiten der Interpretation des Fundbestandes. In: Karwowski, M. - Salač, V. - Sievers, S. /eds./: Boier zwischen Realität und Fiktion. Akten des internationalen Kolloquiums in Český Krumlov vom 14.-16. 11. 2013. Kolloquien zur Vor- und Frühgeschichte 21. Bonn, 77-109.

Militký, J. 2016:

The Coins of the Taurisci and Norici Found in Bohemia and Moravia. In: Karwowski, M. - Ramsl, P. C. /eds./: Boii - Taurisci. Proceedings of the International Seminar, Oberleis-Klement, June $14^{\text {th }}-15^{\text {th }}, 2012$. Mitteilungen der Prähistorischen Kommission 85. Wien, 89-115.

Militký, J. 2018:

Celtic coins from the central site of Žehuň. Památky archeologické 109, 179-231.

Militký, J. 2019:

Keltské mincovnictví ve 3. a 2. století před Kristem v Čechách — Keltisches Münzwesen im 3. und 2. Jahrhundert vor Christus in Böhmen. Praha ( $2^{\text {nd }}$ edition, supplemented and adjusted).

Militký, J. 2020:

Keltisches Münzwesen in Nordostösterreich. In: Trebsche, P. /ed./: Keltische Münzstätten und Heiligtümer. Die jüngere Eisenzeit im Osten Österreichs (ca. 450 bis 15 v. Ch.). Archäologie Niederösterreichs, Band 7. Wien, 294-321.

Militký, J. - Beneš, Z. 2016:

Keltské mince $z$ předhradí hradiště Kolo u Týnce nad Labem (okr. Kolín) - The Celtic coins from the bailey of the castellum called Kolo near Týnec nad Labem (Kolín district). Numismatický sborník 30/2, 234-266.

Militký, J. - Karwowski, M. 2013:

Gold und Silber bei den Boiern und ihren südöstlichen Nachbarn - numismatische und archäologische Überlieferung. In: Hardt, M. - Heinrich-Tamáska, O. /eds./: Macht des Goldes, Gold der Macht. Herrschafts- und Jenseitsrepräsentation zwischen Antike und Frühmittelalter im mittleren Donauraum. Forschungen zu Spätantike und Mittelalter 2. Weinstadt, 17-31.

Militký, J. - Vacinová, L. 2012:

Keltské, římské a raně byzantské mince (3. století před Kristem - 7. století po Kristu) - Celtic, Roman and early Byzantine Coins ( $3^{\text {rd }}$ century $\mathrm{BC}-7^{\text {th }}$ century $\mathrm{AD}$ ). Národní muzeum Chaurova sbírka, díl X. Praha.

Moreno, M. /ed./ 2003:

Delle medaglie carnicho-illiriche del P. Angelo Maria Cortenovis. Villa Manin.

Musilová, M. 2012:

Antické sklo z Bratislavského hradu - Zimná jazdiareň. In: Březinová, G. - Varsik, V. /eds. /: Archeológia na prahu histórie. K životnému jubileu Karola Pietu. Nitra, 363-374. 
Musilová, M. - Barta, P. - Herucová, A. /eds./ 2014:

Bratislavský hrad. Dejiny, výskum a obnova. Bratislava.

Musilová, M. - Kolníková, E. - Hložek, M. 2015:

„Rímska stavba I“ na akropole bratislavského keltského oppida - svedectvo mincí - 'Roman building I' at the Celtic oppidum's acropolis of the Bratislava Castle - testimony of coins. Slovenská archeológia 63, 239-308.

Münzzentrum Rheinland 145:

Münzzentrum Rheinland. Auktion 145, 2008.

Nemeškalová-Jiroudková, Z. 1970:

Athénská mince nalezená v Jihlavě. Numismatický sborník 11 (1969-1970), 179.

Nemeškalová-Jiroudková, Z. 1975:

Keltské zlaté mince $z$ Boiohaema nalezené ve střední Itálii Keltische Goldprägungen aus böhmischen Münzstätten in Mittelitalien. Památky archeologické 66, 383-416.

Nemeškalová-Jiroudková, Z. 1998:

Keltský poklad ze Starého Kolína - Der Keltenschatz in Starý Kolín. Praha.

Ondrouch, V. 1958:

Keltské mince typu Biatec $z$ Bratislavy. Poklad vel'kých strieborných mincí z roku 1942 — Die keltischen Münzen vom Biatec-Typus aus Bratislava. Der Tetradrachmen-Schatzfund aus dem Jahre 1942. Bratislava.

Ondrouch, V. 1961:

Mince Keltov a Geto-Dakov na Slovensku — Münzen der Kelten und Dako-Geten in der Slowakei. Moravské numismatické zprávy 8, 5-36

Ondrouch, V. 1964:

Nálezy keltských, antických a byzantských mincí na Slovensku I. - Finds of Celtic, Antique and Byzantian Coins in Slowakia I. Bratislava.

Paulsen, R. 1933:

Die Münzprägungen der Boier. Leipzig - Wien.

Pieta, K. 2008:

Keltské osídlenie Slovenska. Mladšia doba laténska. Nitra.

Pieta, K. - Zachar, L. 1993:

Mladšia doba železná (laténska). In: Štefanovičová, T. a kol.: Najstaršie dejiny Bratislavy. Bratislava, 143-209.

Pink, K. 1932:

Ein bojisches Großsilberstück aus Spitz a. d. Donau. Numismatische Zeitschrift 25, 9-15.

Pink, K. 1974:

Einführung in die keltische Münzkunde. Wien.

Plachá, V. - Fiala, A. 1998:

Hromadný nález keltských mincí na hrade Devín - Hortfund keltischer Münzen auf der Burg Devín. Slovenská numizmatika 15, 201-210.

Price, M. J. 1991:

The Coinage in the Name of Alexander the Great and Philip Arrhidaeus. A British Museum Catalogue. Zurich - London.

Prokopov, I. 2006:

Die Silberprägung der Insel Thasos und die Tetradrachmen des „thasischen Typs“ vom 2.-1. Jahrhundert v. Chr. Berlin.

\section{Resutík, B. 2016:}

Rímská stavba II vo svetle antického stredomorského importu v keltskom prostredí a torzá stavieb III., IV. a VI. L'edificio romano II alla luce delle antiche importazioni attraverso il Mediteraneo in ambiente celtico e resti degli edifici III, IV e VI. In: Musilová, M. et al. /eds./: Kelti z Bratislavy. Bratislava, 95-115.
Röttger, Ch. 2013:

DEVII, FAPIARIX und FAPIARIX retrograd. Vorschlag zur korrekten Lesung einiger Münzlegenden. Numismatický sborník 27/1 (2012-2013), 27-33.

Röttger, Ch. 2015:

Überlegungen zur Zeitstellung der Münzen der Biatec-Reihe Considerations about dating the coins of the Biatec-series. Zborník Slovenského národného múzea 109 - Archeológia 25, 195-209.

Röttger, Ch. 2019:

Corpvs Nvmmorvm Biateci Et Sociorvm Eivs (CNB). CD-ROM Database.

Röttger, Ch. 2020:

Der Fund von Bratislava aus dem Jahr 1776. Denarius 8, 20-27.

$R R C$ :

Crawford, M. H. 1974:

Roman Republican Coinage I-II. Cambridge.

Rudnicki, M. 2011:

Nieznana moneta celtyckich Bojów z Grójca (część miasta Konina). Wiadomości Numizmatyczne 55, 99-132.

Ruske, A. 2011a:

Die Fundregesten der antiken Münzhorte aus Österreich. Eine Zusammenstellung aller bekannten Münzdepots anhand der Literatur. Numismatische Zeitschrift 118, 53-181.

Ruske, A. 2011 b:

Der keltischen Münzhort von Schottwien (1902). Mitteilungen der Österreichischen Numismatischen Gesellschaft 31, 132-138.

Sacken, E. Freiherr v. 1862:

Über die vorchristlichen Culturepochen Mitteleuropa's und die Quellen der deutschen Urgeschichte. Wien.

Schmeller, A. 1965 :

Das Burgenland: seine Kunstwerke, historischen Lebens- und Siedlungsformen. Österreichische Kunstmonographie, Band 3. Salzburg.

Schröer, J. 1855

Miszellen. Österreichische Blätter für Literatur und Kunst 41, 8. 10. 1855, 307 .

Seidl, J. G. 1855:

V. Beiträge zu einer Chronik der archaeologischen Funde in der österreichischen Monarchie. Archiv für Kunde österreichischer Geschichtsquellen 15, 241-336.

Sotheby 1923:

Catalogue of the important and representative collection of Celtic coins, of the Rhine and Danube districts, Celtiberian and Gaulish; Greek coins, chisel-cut and defaced coins, imitations of Roman Denarii, \&c. formed during a period of over twenty years by the late Horace Sandars, Esq. F.S.A., of 10H, Queen Anne's Mansions, Westminster, S.W.1. by Sotheby, Wilkinson \& Hodge, 12. 2. 1923.

\section{Szilágyi, S. 1895:}

A magyar nemzet története. Első kötet. Magyarország a királyság megalapitásáig. Budapest.

Ščasnár, V. - Kliment, V. - Červeňanský, M. - Zachar, L. 1984: Zlomky dávkovacích platničiek na výrobu keltských mincí - nálezy zo Šaštína-Stráží a Bratislavy - Bruchstücke von Dosierplätchen zur Herstellung keltischer Münzen - Funde aus Šaštín-Stráže und Bratislava. Slovenská numizmatika 8, 121-145.

Šedivý, J. - Štefanovičová, T. /eds. / 2012:

Dejiny Bratislavy 1. Od počiatkov do prelomu 12. a 13. storočia. Bratislava. 
Thompson, M. 1961:

The New Style Silver Coinage of Athens. The American Numis matic Society. Numismatic Studies 10. New York.

Torbágyi, M. 1984:

Die Münzprägung der Eravisker. Acta Archeologica Academie Scientiarum Hungariae 36, 161-171.

Torbágyi, M. 1992:

Késő kelta pénzverés Magyarországon. Az érem 1992/2, 1-8.

Torbágyi, M. 1997:

Keltische Münzprägung im Gebiet Ungarns. Numismatische Zeitschrift 104-105, 7-17.

Torbágyi, M. 2008:

Questions of coin use in the late celtic Transdanubia. Cercetări numismatice $14,203-212$.

Torbágyi, M. - Vida, I. A. 2020:

Some Remarks on Eraviscan Coinage. Notae Numismaticae / Zapiski Numizmaticzne 15. Kraków, 49-64.

Vrtel, A. 2011 :

Neskorolaténsky objekt 115/09 na nádvorí bratislavského hradu - La structure No 115/09 dans la cour du château de Bratislava (période de La Tène finale). In: Droberjar, E. /ed./ Archeologie barbarů 2010. Hroby a pohřebiště Germánů mezi Labem a Dunajem. Sborník příspěvků ze VI. Protohistorické konference Hradec Králové, 6.-9. září 2010. Olomouc, 265-277.
Vrtel, A. - Maretta, R. G. 2018:

Poklad keltských mincí objavený v Bratislave roku 1776 - lokalizácia miesta nálezu. Historický časopis 66, 409-428.

Waldhauser, J. 1983:

Závěrečný horizont keltských oppid v Čechách (Konfrontace výkladů historických pramenů, numismatiky a archeologie) Schlußhorizont der keltischen Oppida in Böhmen (Konfrontation der Deutung historischer Quellen, der Numismatik und Archäologie). Slovenská archeológia 31/2, 325-356.

Ziegaus, B. 1995:

Der Münzfund von Großbissendorf. Eine numismatisch-historische Untersuchung zu den spätkeltischen Goldprägungen in Südbayern. Ausstellungskataloge der Prähistorischen Staatssammlung, Band 27. München.

Ziegaus, B. 2010:

Kelten Geld. Münzen der Kelten und angrenzender nichtgriechischer Völkerschaften. Sammlung Christian Flesche. München.

Ziegaus, B. 2013:

Münzen und Münzwerkzeuge. Katalog der Münzen und Werkzeuge. In: Sievers, S. - Leicht, M. - Ziegaus, B.: Ergebnisse der Ausgrabungen in Manching-Altenfeld 1996-1999. Die Ausgrabungen in Manching, Band 18, Teil 2: Text. Wiesbaden, 425-619.

Doc. PhDr. Jiří Militký, Ph.D., DSc., National Museum - Historical Museum, Numismatic department (Národní muzeum - Historické muzeum, Numismatické oddělení), Vinohradská 1, 11000 Praha 1, Czech Republic;

e-mail: militky.jiri@seznam.cz

Dr. Melinda Torbágyi, Ph.D., Hungarian National Museum (Magyar Nemzeti Múzeum), Múzeum krt. 14-16, 1088 Budapest, Hungary; e-mail: torbagyi@hnm.hu 
